\title{
Laserinduzierte Änderungen der elektronischen Transporteigenschaften von Manganaten
}

\author{
Dissertation \\ zur Erlangung \\ des mathematisch-naturwissenschaftlichen Doktorgrades \\ „Doctor rerum naturalium" \\ der Georg-August-Universität Göttingen \\ im Promotionsprogramm ProPhys \\ der Georg-August University School of Science (GAUSS) \\ vorgelegt von \\ Manuel Mounir Mchalwat \\ aus Duderstadt
}

Göttingen, 2017 


\section{Betreuungsausschuss}

Prof. Dr. Konrad Samwer

I. Physikalisches Institut, Georg-August-Universität, Göttingen

Prof. Dr. Markus Münzenberg

Institut für Physik, Ernst-Moritz-Arndt-Universität, Greifswald

Prof. Dr. Christian Jooß

Institut für Materialphysik, Georg-August-Universität, Göttingen

\section{Mitglieder der Prüfungskommission}

Referent: Prof. Dr. Konrad Samwer

I. Physikalisches Institut, Georg-August-Universität, Göttingen

Korreferent: Prof. Dr. Markus Münzenberg

Institut für Physik, Ernst-Moritz-Arndt-Universität, Greifswald

weitere Mitglieder der Prüfungskommission:

Prof. Dr. Christian Jooß

Institut für Materialphysik, Georg-August-Universität, Göttingen

Prof. Dr. Vasily Moshnyaga

I. Physikalisches Institut, Georg-August-Universität, Göttingen

Prof. Dr. Hans Christian Hofsäss

II. Physikalisches Institut, Georg-August-Universität, Göttingen

Prof. Dr. Michael Seibt

IV. Physikalisches Institut, Georg-August-Universität, Göttingen

Tag der mündlichen Prüfung: 9. Mai 2017 


\section{Inhaltsverzeichnis}

$\begin{array}{ll}1 . \text { Einleitung } & 1\end{array}$

2. Theoretische Grundlagen 5

2.1. Manganate ........................... 5

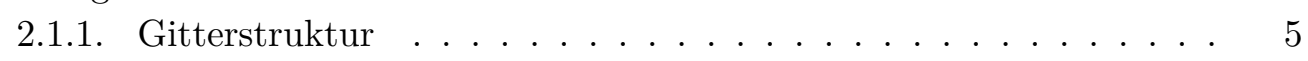

2.1.2. Elektronische Struktur . . . . . . . . . . . . . 6

2.1.3. Austauschmechanismen . . . . . . . . . . . . . . 8

2.1.4. Polaronen . . . . . . . . . . . . . . . . . . . . . . . . . . . 12

2.1.5. Phasendiagramme . . . . . . . . . . . . . . . . . . 14

2.2. Dritte harmonische Spannung . . . . . . . . . . . . . . . . . . . . 19

$2.3 . \quad$ Optische Anregungen $\ldots \ldots \ldots \ldots \ldots \ldots$

2.3.1. Auswirkungen auf die Gitterstruktur . . . . . . . . . . . 26

3. Experimentelle Methoden und Geräte 29

3.1. Elektronischer Transport im Wechselfeld . . . . . . . . . . . . . . . . . . 29

3.2. Strom-Spannungs-Kennlinien . . . . . . . . . . . . . . . . . . 30

3.3. Frequenzverhalten periodischer Anregung . . . . . . . . . . . . . . . . . 30

3.4. Probenherstellung und -präparation . . . . . . . . . . . . . . . . 31

3.4.1. Metallorganische Aerosol-Deposition . . . . . . . . . . . . . 31

$3.4 .2 . \quad$ Strukturierung . . . . . . . . . . . . . . . . . . . . . . . . . . . . . . . . . . .

$3.4 .3 . \quad$ Kontaktierung $\ldots \ldots \ldots \ldots$. . . . . . . . . . . . . . 33

3.5. Laseranregung . . . . . . . . . . . . . . . . . . . . . . . . . 33

3.5.1. Funktionsweise eines Titan:Saphir-Lasers . . . . . . . . . . . 34

3.5.2. Femtolasers Femtosource fusion $20 \ldots \ldots$. . . . . . . . . 34

3.5.3. Coherent RegA 9040 . . . . . . . . . . . . . . . . . 36

3.6. Zeitliche Mittelung der Messgrößen . . . . . . . . . . . . . . . . 36

3.7. PPMS-Messeinsatz . . . . . . . . . . . . . . . . . . . . . 37

3.7.1. Messeinsatz . . . . . . . . . . . . . . . . . . . . 38

3.7.2. Vakuumdurchführung des photonischen Kristalls . . . . . . . . 40

3.7.3. Kalibrierung des Thermometers . . . . . . . . . . . . . . . . 41

3.8. Probenhalter mit Peltier-Kühlung . . . . . . . . . . . . . . . . . 42

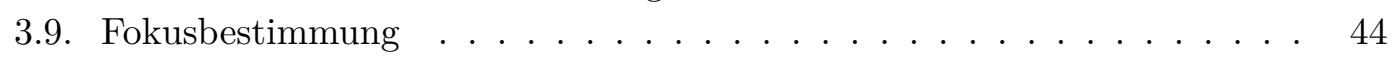

3.10. Abbilden der Probenoberfläche . . . . . . . . . . . . . . . . 45 
\begin{tabular}{|l|l}
\hline 4. Experimentelle Ergebnisse & 47
\end{tabular}

4.1. Lanthan-Barium-Manganat . . . . . . . . . . . . . . . . . . . 47

4.1.1. Grundlegende Charakterisierung . . . . . . . . . . . . . . 47

$4.1 .2 . \quad$ Frequenzanalyse . . . . . . . . . . . . . . . . . 52

$4.1 .3 . \quad$ Bestimmung des Fokus . . . . . . . . . . . . . . . . . . . 53

$4.1 .4 . \quad$ Positionierung $\ldots \ldots \ldots \ldots \ldots \ldots$

4.1.5. Einfluss von Laseranregung auf die dritte harmonische Spannung 55

4.1.6. Abhängigkeit der harmonischen Spannungen von der Anregungs-

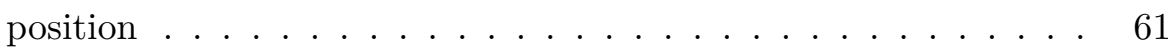

4.1.7. Wärmedissipation . . . . . . . . . . . . . . . 63

4.1.8. Strom-Spannungs-Kennlinien . . . . . . . . . . . . . . . 63

4.2. Lanthan-Praseodym-Calcium-Manganat . . . . . . . . . . . . . . . . 64

4.2.1. Grundlegende Charakterisierung . . . . . . . . . . . . . . . 65

$4.2 .2 . \quad$ Positionierung $\ldots \ldots \ldots \ldots \ldots$

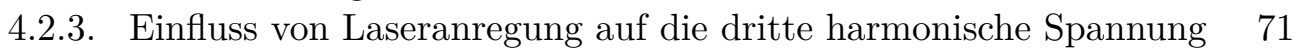

$\begin{array}{ll}\text { 5. Diskussion } & \mathbf{7 5}\end{array}$

5.1. Kontakte . . . . . . . . . . . . . . . . 75

5.2. Strom-Spannungs-Kennlinie . . . . . . . . . . . . . . . . 75

5.3. Wärmedissipation . . . . . . . . . . . . . . . . . . . 79

5.4. Einfluss von Laseranregung auf die dritte harmonische Spannung . . . . 82

5.4.1. Skalierung des gemessenen Effekts aufgrund zeitlicher Mittelung 84

5.5. Abhängigkeit von der Anregungsposition . . . . . . . . . . . . . 86

6. Zusammenfassung und Ausblick 89

\begin{tabular}{ll}
\hline A. Anhang & 91
\end{tabular}

A.1. Zusätzliche Graphen . . . . . . . . . . . . . . . . . . . . . . . . 91

A.2. Abgrenzung der Messmethode . . . . . . . . . . . . . . . . . . . . . . . . 100

\begin{tabular}{ll}
\hline Literatur & 101
\end{tabular}

\begin{tabular}{lr}
\hline Publikationen & 111
\end{tabular}

\begin{tabular}{ll}
\hline Danksagung & 113
\end{tabular} 


\section{Abbildungsverzeichnis}

2.1. Perowskit-Struktur der Manganate und Ionenradien . . . . . . . . . . 6

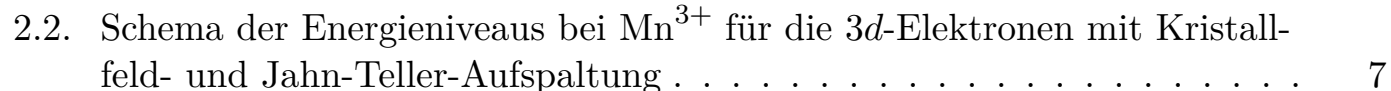

2.3. Schema zur Veranschaulichung der GKA-Regeln beim Superaustausch • 10

2.4. Schema zur Veranschaulichung des Doppelaustauschs . . . . . . . . . . . 11

2.5. Schemata verschiedener Polaronen . . . . . . . . . . . . . . . . . 13

2.6. Stark vereinfachtes Schema zur Veranschaulichung korrelierter Polaronen 14

2.7. Generisches Phasendiagramm der Manganate . . . . . . . . . . . . 15

2.8. Phasendiagramm von LBMO . . . . . . . . . . . . . . . . . . . . . . . . 16

2.9. Phasendiagramm von LPCMO . . . . . . . . . . . . . . . . . . . 18

2.10. Dritter harmonischer Koeffizient für einige Manganate . . . . . . . . . . 20

2.11. Optische Leitfähigkeit eines PCMO-Films . . . . . . . . . . . . . . . 23

2.12. Schematische Darstellung der komplexen dielektrischen Funktion . . . . 24

2.13. Komplexer Brechungsindex eines PCMO-Films . . . . . . . . . . . 25

2.14. Gesamtenergie von $\mathrm{LaMnO}_{3}$ in der paramagnetischen Hochtemperaturphase in Abhängigkeit der Jahn-Teller-Verzerrung . . . . . . . . . . 27

3.1. Schema der Messgeometrie für elektrischen Transport im Wechselfeld . . 29

3.2. Schema der elektrischen Messungen im Gleichfeld . . . . . . . . . . . . 30

3.3. Schema des Messaufbaus zur Bestimmung der Probenantwort auf periodische Laseranregung . . . . . . . . . . . . . . . . . . . . . 31

3.4. Schema der MAD-Anlage zur Herstellung von dünnen Filmen . . . . . . 31

3.5. Schema der Box zum Einkoppeln in den photonischen Kristall . . . . . . 35

3.6. Schema des Lasersystems Coherent RegA 9040 . . . . . . . . . . . . . . 36

3.7. Charakteristische Zeiten der Pulse bei den verwendeten Lasern . . . . . 37

3.8. Schema des PPMS-Probenkopfes . . . . . . . . . . . . . . . . 38

3.9. Foto des Messeinsatzes . . . . . . . . . . . . . . . . . . . . . . 39

3.10. CAD-Zeichnung und Foto des Messkopfes . . . . . . . . . . . . . . . . 39

3.11. Freigelegter photonischer Kristall . . . . . . . . . . . . . . . . . . . . . . 41

3.12. Eingeklebte Glasfaser für die Vakuumdurchführung . . . . . . . . . . . . . . . 41

3.13. Kalibrierung des Cernox-Thermometers . . . . . . . . . . . . . . . . 42

3.14. Scheinbare relative Temperaturänderung des Cernox-Thermometers durch

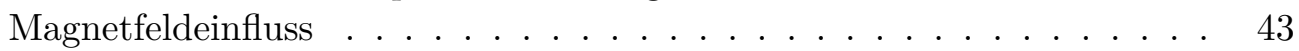

3.15. CAD-Zeichnung des Probenhalters mit Peltier-Kühlung . . . . . . . . . 43

3.16. Strahlteiler im Messkopf . . . . . . . . . . . . . . . . . 46 
4.1. Phasendiagramm von LBMO . . . . . . . . . . . . . . . . . . . . . . 48

4.2. Röntgendiffraktometrie von LBMO . . . . . . . . . . . . . . . . . . . 48

4.3. $\quad$ Rastertunnelmikroskop-Aufnahme der LBMO-Probe . . . . . . . . . . . 48

4.4. Foto der Struktur auf der LBMO-Probe . . . . . . . . . . . . . . . . . . 49

4.5. Temperaturabhängigkeit des Widerstandes der LBMO-Probe . . . . . . 50

4.6. Magnetisierung in Abhängigkeit der Temperatur von LBMO . . . . . . . 51

4.7. Frequenzanalyse für Messungen der ersten und dritten harmonischen

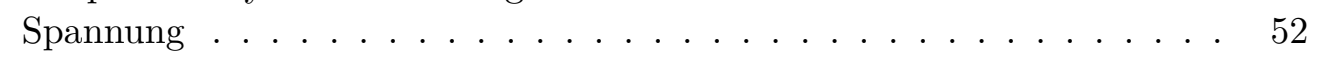

4.8. Probenoberfläche der Probe, dargestellt durch die Thermospannung . . 54

4.9. Karte der Probenoberfläche anhand der Reflektivität . . . . . . . . . . . 55

4.10. Zeitentwicklung der ersten und dritten harmonischen Spannung unter

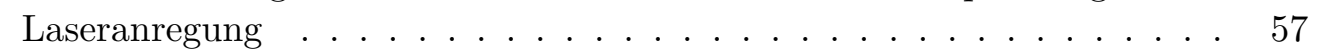

4.11. Temperaturabhängigkeit der ersten und dritten harmonischen Spannung

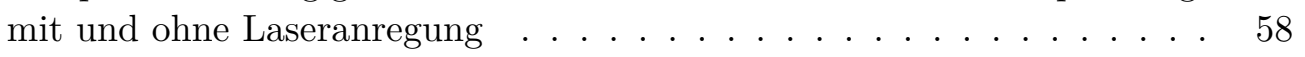

4.12. Lichtinduzierte Änderung des dritten harmonischen Koeffizienten . . . . 59

4.13. Temperaturabhängigkeit der ersten und dritten harmonischen Spannung und des dritten harmonischen Koeffizienten bei Messungen am Lasersystem in Greifswald . . . . . . . . . . . . . . . . . . . . . 60

4.14. Harmonische Spannungen beim Linearscan des Lasers über die Probe. . 61

4.15. Änderung des dritten harmonischen Koeffizienten mit der Anregungsposition und Temperaturverschiebung . . . . . . . . . . . . 62

4.16. Änderung des Gleichstromwiderstandes durch periodische Lasereinwirkung 63

4.17. Strom-Spannungs-Kennlinien . . . . . . . . . . . . . . . . . . 64

4.18. Röntgendiffraktometrie von LPCMO . . . . . . . . . . . . . . . . . 65

4.19. Rastertunnelmikroskop-Aufnahme der LPCMO-Probe . . . . . . . . . . 66

4.20. Schematischer Aufbau der strukturierten LPCMO-Probe . . . . . . . . . 67

4.21. Temperaturabhängigkeit des Gleichstromwiderstandes der LPCMO-Probe 68

4.22. Widerstandskurve der strukturierten LPCMO-Probe als Überlagerung zweier unterschiedlicher Dotierungen $\ldots \ldots \ldots \ldots \ldots$

4.23. Reflektivitätskarte der Probenoberfläche von LPCMO . . . . . . . . . . 70

4.24. Temperaturabhängigkeit der ersten und dritten harmonischen Spannung und des dritten harmonischen Koeffizienten von LPCMO unter Laseran-

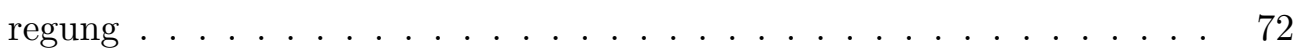

4.25. Änderung des dritten harmonischen Koeffizienten durch Laseranregung bei LPCMO . . . . . . . . . . . . . . . . . . . . . . . . 73

4.26. Magnetfeldabhängigkeit der ersten und dritten harmonischen Spannung und des dritten harmonischen Koeffizienten von LPCMO. . . . . . . . . 74

5.1. Schema der Kontaktbeschaffenheit auf den Proben . . . . . . . . . . . . 76

5.2. Temperaturverlauf des Widerstandes für verschiedene Spannungen bzw.

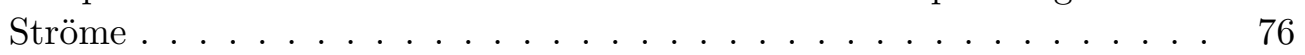

5.3. Joulesche Erwärmung ． . . . . . . . . . . . . . . . . . 77

5.4. Widerstand in Abhängigkeit der Spannung und des Stroms von LBMO. 78 


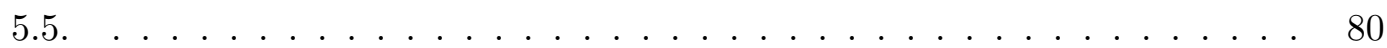

5.6. Zeitlicher Verlauf der Signaländerung aufgrund der Laseranregung . . . 85

5.7. Skalierungsfaktor für den gemessenen Effekt bei verschiedenen Relaxati-

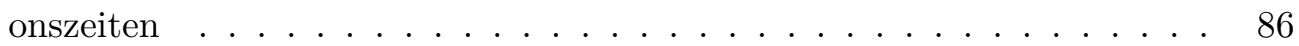

A.1. Schwingverhalten in der dritten harmonischen Spannung . . . . . . . . . 91

A.2. Temperaturabhängigkeit der ersten und dritten harmonischen Spannung von LBMO bei positionsabhängiger Laseranregung in Greifswald . . . . 92

A.3. Zeitserien der LBMO-Probe mit Laseranregung aus Greifswald . . . . . 93

A.4. Zeitserien der LPCMO-Probe mit Laseranregung . . . . . . . . . . . . . 94

A.5. Zeitserien der LPCMO-Probe mit Laseranregung im Magnetfeld . . . . 95

A.6. Widerstand in Abhängigkeit von Strom und Spannung von LBMO für ausgewählte Temperaturen . . . . . . . . . . . . . . . 96

A.7. Widerstand in Abhängigkeit von Strom und Spannung von LBMO für ausgewählte Temperaturen, Logarithmische Auftragung des positiven Astes 97

\begin{tabular}{|lll}
\hline A.8. Widerstände der äußeren Kontakte bei der Vierpunktmessung an LBMO 98 & 98
\end{tabular}

A.9. Normierte Änderung des Gleichstromwiderstandes bei periodischer La-

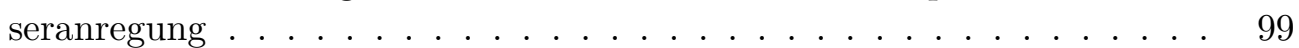

\section{Tabellenverzeichnis}

5.1. Vergleich der Leistungsdaten der Lasersysteme . . . . . . . . . . . . . 82 



\section{Liste der Abkürzungen}

LBMO Lanthan-Barium-Manganat - $\mathrm{La}_{1-x} \mathrm{Ba}_{x} \mathrm{MnO}_{3}$

LPCMO Lanthan-Praseodym-Calcium-Manganat - $\left(\mathrm{La}_{1-y} \mathrm{Pr}_{y}\right)_{1-x} \mathrm{Ca}_{x} \mathrm{MnO}_{3}$

LCMO Lanthan-Calcium-Manganat $-\mathrm{La}_{1-x} \mathrm{Ca}_{x} \mathrm{MnO}_{3}$

PCMO Praseodym-Calcium-Manganat - $\mathrm{Pr}_{1-x} \mathrm{Ca}_{x} \mathrm{MnO}_{3}$

LSMO Lanthan-Strontium-Manganat - $\mathrm{La}_{1-x} \mathrm{Sr}_{x} \mathrm{MnO}_{3}$

LMO Lanthan-Manganat - $\mathrm{LaMnO}_{3}$

STO Strontiumtitanat $-\mathrm{SrTiO}_{3}$

PPMS Physical Properties Measurement System

MAD Metallorganische Aerosol-Deposition

DMF Dimethylformamid

TEM Transmissionselektronenmikroskop(ie)

FIB Focused Ion Beam

FM Ferromagnetismus/ferromagnetisch

AFM Antiferromagnetismus/antiferromagnetisch

GKA Goodenough-Kanamori-Anderson

CMR Colossal Magnetoresistance

TCR Temperature Coefficient of Resistance

FWHM Full Width Half Maximum 



\section{Einleitung}

Viele Errungenschaften unserer modernen Gesellschaft und zu einem großen Teil auch der heute erreichte Wohlstand sind die Folge einer technologischen Revolution aus der Mitte des vergangenen Jahrhunderts. Denn 1947 gelang Shockley, Bardeen und Brattain BB48 die erste Realisierung eines Bipolartransistor 1 basierend auf einem Halbleiter-Materia $2^{2}$, welcher als Verstärker oder in anderer Funktion die Vakuumröhren ersetzen konnte. Aber aufgrund der langsamen Schaltzeit gegenüber den Röhren konnte sich dieser Transistor zunächst nicht durchsetzen. Erst der auf einem leicht anderen Prinzip beruhende Feldeffekttransistor (FET) fand dann zunehmend Verwendung $5^{3}$ und führte schließlich zu der heutigen Ubiquität von integrierten Schaltkreisen auf Basis des Halbleiters Silizium in fast allen Bereichen der westlichen Welt. Dabei haben die Erfindungen, die dieser Halbleiter als funktionales Material ermöglicht hat, unsere Gesellschaft so gravierend verändert, dass manche die entsprechende Epoche bereits als Siliziumzeitalter bezeichnen [Ros97]. Und in naher Zukunft werden Automatisierung und vor allem künstliche Intelligenz unsere Arbeitswelt drastisch umgestalten. [Sch16] Sogar die Wissenschaft selbst wurde durch die digitale Datenverarbeitung und durch Simulationen auf Hochleistungsrechnern stark in positiver Weise beeinflusst.

Nun ist bei dieser Materialklasse die Entwicklung dermaßen fortgeschritten und dadurch die Größe der funktionalen Strukturen, also vornehmlich der Transistoren, so weit geschrumpft, dass bei weiterer Reduktion ein vollständiger Sperrzustand kaum mehr zu erreichen oder es aufgrund der benötigten niedrigeren Betriebsspannung nicht mehr möglich ist, hohe Ströme zu schalten [Lun03]. Denn bei diesen Größenordnungen, wo ein Gate nur noch aus einigen zehn Atomen besteht, kommen quantenmechanische Effekte wie das Tunneln immer stärker zum Tragen [KSB00 Lui+11]. Daher ist die Forschung schon auf der Suche nach neuen Ansätzen für derart kleine Strukturen und in der Tat konnten mithilfe von Kohlenstoffnanoröhrchen bereits Gatelängen von $1 \mathrm{~nm}$ realisiert werden Des+16.

Ein anderer Weg ist es, die Materialklasse grundlegend zu wechseln und die gewünschten Funktionalitäten auf andere Weise zu erreichen. Dort könnten die komplexen

\footnotetext{
${ }^{1}$ Dafür erhielten sie 1956 den Nobelpreis in Physik.

${ }^{2}$ Es gab sowohl eine Ausführung mit n-dotiertem Silizium als auch mit n-dotiertem Germanium, welche beide jeweils an der Oberfläche, wo Emitter und Kollektor als Spitzen den Kontakt bildeten, durch eine chemische oder andere Behandlung p-dotierten Charakter erhielten, während die Basis-Elektrode weiterhin n-dotiert blieb. Somit handelte es sich also um einen PNP-Transistors.

${ }^{3}$ Tatsächlich wurde der Feldeffekttransistor vor dem Bipolartransistor erdacht, denn theoretische Beschreibungen reichen bis in die 1920er Jahre zurück. Aber aufgrund der schwierigen technischen Umsetzung dauerte es über dreißig Jahre, bis 1959 der erste FET gezeigt werden konnte und sein Siegeszug begann. Kah76.
} 
Metalloxide - wobei es sich im Fall dieser Arbeit um Manganate, also Manganoxide in Perowskit-Struktur handelt - aufgrund ihrer vielschichtigen Phänomene für manche

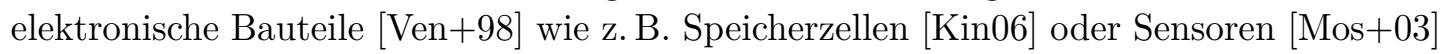
eine Alternative bereitstellen. So findet man in diesen Materialien magnetische Ordnungen wie Ferro- oder Antiferromagnetismus, aber auch das elektrische Analogon: die Ferroelektrizität, weiterhin metallische oder isolierende Zustände. Und fast immer treten diese Eigenschaften im Zusammenspiel miteinander bzw. in Konkurrenz gegeneinander auf. Dabei gibt es vielfältige Möglichkeiten, diese Effekte zu beeinflussen, sei es durch intrinsische Veranlagung oder durch externe Anregung, auf welche die Systeme zum Teil extrem empfindlich reagieren. Erstere ergibt sich vor allem durch die Elementauswahl und Dotierung, Letztere umfassen unter anderem Temperatur, Druck [Mos+00], Richtung und Stärke äußerer Magnetfelder [Hel+93], elektrische Felder, die auch ein Stromfluss durch das Material hervorrufen kann [Asa+97], und elektromagnetische Felder, zu denen natürlich auch Licht gehört Miy+97, Fie+98. Diese Stimuli können die Freiheitsgrade wie Ladung, Spin, Gitter und deren Kopplungen nachhaltig beeinflussen, sodass sich im Material andere Phasen oder Zustände einstellen [MS11]. Eine der bekanntesten dieser äußeren Einflussnahmen in Manganaten ist die Unterdrückung des Widerstandes um mehrere Größenordnungen durch ein externes Magnetfeld [Jin+94], was man als kolossalen Magnetowiderstand (CMR für engl. Colossal Magnetoresistance) bezeichnet Ram97. Weiteres bemerkenswertes Verhalten bestimmter Manganate ist ein von der elektrischen Vorgeschichte abhängiger Widerstand, was bedeutet, dass dieser durch den Fluss eines ausreichend großen Stromes von einem hohen in einen niedrigen Zustand und umgekehrt geschaltet werden kann; man spricht von einem Memristor Kal+11; Mos+10]. Außerdem kann man unter besonderen Voraussetzungen in perowskitischen Metalloxiden sogar Supraleitung beobachten [Rey+07] und es gibt noch viele andere interessante Erscheinungen in dieser Materialklasse [MS07; Dag05].

In der hier vorliegenden Arbeit wurde nun der Einfluss von lokalen Anregungen durch kurze Laserpulse auf stark korrelierte Manganate anhand ihrer Transporteigenschaften untersucht und dabei im Besonderen die Nichtlinearitäten betrachtet, mithilfe derer sich Rückschlüsse auf die Korrelationen zwischen den Elektronen und den Phononen und damit dem Gitter ziehen lassen.

Dabei werden in Kapitel 2 die Manganate allgemein eingeführt und grundlegende Eigenschaften wie Gitter- und elektronische Struktur vorgestellt, wobei darauf aufbauend Polaronen erläutert werden, die als ein Urheber des nichtlinearen Verhaltens gelten. Anschließend werden auch die beiden hier untersuchten Systeme Lanthan-BariumManganat (LBMO) und Lanthan-Praseodym-Calcium-Manganat (LPCMO) bezüglich ihrer magnetischen, elektrischen und Gitterstruktur beschrieben und ihre Position im generischen Phasendiagramm diskutiert. Danach wird die dritte harmonische Spannung als spezielle Messgröße eingeführt und ihr Zusammenhang mit korrelierten Polaronen hergeleitet, bevor am Ende des Abschnitts optische Eigenschaften und Absorptionen für die Manganate erörtert werden.

Kapitel 3 beschreibt die technischen Aspekte, also die verwendeten Messgeräte, -geometrien und Techniken, aber auch die Art der Probenherstellung mittels der Metall- 
organischen Aerosol-Deposition (MAD) und die Mikrostrukturierung durch Lithographie, was beides von Kollegen im Institut übernommen wurde. Ebenso werden die verwendeten Titan-Saphir-Laser zur Erzeugung von Pulsen mit Längen im Femtosekundenbereich aus Göttingen und Greifswald vorgestellt und kurz ihre Funktionsweise erklärt. Einen großen Teil nimmt dann die Beschreibung der Konstruktion und der Eigenschaften eines Messeinsatzes für einen kommerziellen Kryostaten ein, welcher während dieser Arbeit eigenständig konzipiert und entwickelt wurde. Das umfasste die CAD-Zeichnung des Messkopfes sowie die Auswahl optischer Bauteile wie Linsen und Spiegel und das Herstellen einer Vakuumdurchführung für eine spezielle Glasfaser, aber auch die elektrische Verkabelung und Programmierung der Steuer- und Messsoftware. Außerdem wird kurz der zweite Probenhalter für Messungen auf einem optischen Tisch und daher mit beschränkter Kühlmöglichkeit beschrieben, der mit Unterstützung durch das technische Personal im Institut konzipiert wurde.

Alle durchgeführten relevanten Messungen beschreibt und zeigt dann das Kapitel 4 für die Probensysteme. Dazu war zunächst die grundlegende Charakterisierung beider Proben in Bezug auf strukturelle, elektrische und magnetische Eigenschaften und die Wahl bzw. Bestimmung geeigneter Messparameter wie der Frequenz für die elektrische Wechselfeldmessung bzw. der Strahltaille der Laseranregung erforderlich. Anschließend konnte durch Abbilden der Probenoberfläche mithilfe des Lasers die richtige Anregungsposition gefunden und damit die Messung der dritten harmonischen Spannung durchgeführt und der Einfluss des Lasers untersucht werden. Weiterhin wurden noch Strom-Spannungs-Kennlinien und die Wärmedissipation der Laserenergie in das System betrachtet.

In Kapitel 5 werden schließlich die Ergebnisse zusammenfassend bewertet, um Schlussfolgerungen daraus zu ziehen und eine Interpretation zu entwickeln. Dabei ist eine zentrale Frage, wie in den Probensystemen in Abhängigkeit der Laserintensität korrelierte Polaronen erzeugt oder vernichtet werden können.

Am Ende fasst das Kapitel $\mathbf{6}$ noch einmal die zentralen Schlüsse zusammen und gibt einen Ausblick für mögliche weitere Untersuchungen. 



\section{Theoretische Grundlagen}

Im folgenden Kapitel soll das untersuchte Probensystem bezüglich seiner grundlegenden und für diese Arbeit wichtigen Eigenschaften vorgestellt werden, ebenso wie der theoretische Hintergrund zu der Messmethode und den optischen Anregungen in diesen Systemen.

\subsection{Manganate}

Bei den untersuchten Materialien handelt es sich um oxidische Manganatverbindungen, deren physikalische Eigenschaften stark von den elektronischen 3d-Zuständen der Manganelektronen und den damit verbundenen Valenzzuständen der Manganionen abhängen. Diese wiederum werden durch Art und Quantität der außer dem Sauerstoff vorhandenen Gitterionen bestimmt und können zusätzlich durch äußere Parameter wie Temperatur, Magnetfeld, Druck oder durch elektromagnetische Anregungen beeinflusst werden. Eines der bekanntesten Beispiele dafür ist der so genannte kolossale Magnetowiderstands-Effekt (CMR-Effekt, für engl. Colossal Magnetoresistance), bei dem sich der durch Ladungsordnung entstehende riesige Widerstand durch das Anlegen eines äußeren Magnetfeldes um viele Größenordnungen unterdrücken lässt [Hel+93 Jin+94 Ram97], was mit einer auftretenden elektronischen Phasenseparation einhergeht [Ueh+99|. So ist die in den Manganaten entstehende Physik äußerst reichhaltig und umfasst Phänomene, die auf zeitlichen und räumlichen Ausdehnungen über mehrere Größenordnungen auftreten.

\subsubsection{Gitterstruktur}

Manganate kristallisieren in einer Struktur namens Perowski 1 ?1 deren kubische Grundstruktur in Abb. 2.1 dargestellt ist. Dort besetzen nach der allgemeinen Summenformel $\mathrm{ABO}_{3}$ Elemente der so genannten seltenen Erden oder der Erdalkalimetalle die A-Plätze, wobei erstere trivalent, d. h. in der Oxidationsstufe +3 , und letztere divalent, also in +2 auftreten. Wie eingangs schon erwähnt, ist hierbei gerade das Verhältnis aus triund divalenten Kationen auf den $A$-Gitterplätzen entscheidend, denn die Valenz des Manganions auf der zentralen Position $B$ ergibt sich dementsprechend $\mathrm{zu} \mathrm{Mn}^{3+}$ oder $\mathrm{Mn}^{4+}$, was weitreichende Konsequenzen hat, wie sich später zeigen wird. Dabei wird es von sechs Sauerstoffionen $\mathrm{O}^{2-}$ umschlossen, die ein Oktaeder bilden. Jedoch bleibt unter anderem durch die unterschiedlich großen Ionenradien die kubische Struktur nicht

\footnotetext{
${ }^{1}$ Die Bezeichnung stammt von dem Mineral $\mathrm{CaTiO}_{3}$, welches im Erdmantel vorkommt und nach dem russischen Mineralogen Lew Alexejewitsch Perowski benannt ist.
} 

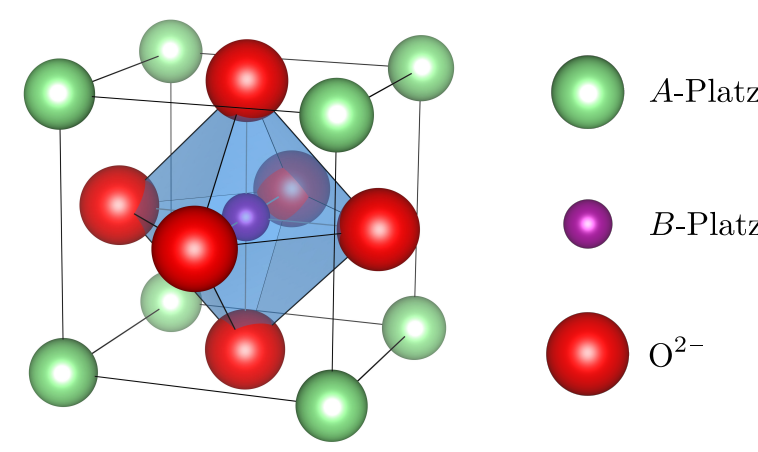

\begin{tabular}{cc}
\hline Ion & Radius \\
\hline $\mathrm{La}^{3+}$ & $1.22 \AA$ \\
$\mathrm{Pr}^{3+}$ & $1.16 \AA$ \\
$\mathrm{Ca}^{2+}$ & $1.06 \AA$ \\
$\mathrm{Sr}^{2+}$ & $1.27 \AA$ \\
$\mathrm{Ba}^{2+}$ & $1.43 \AA$ \\
$\mathrm{Mn}^{3+}$ & $0.70 \AA$ \\
$\mathrm{Mn}^{4+}$ & $0.52 \AA$ \\
$\mathrm{O}^{2-}$ & $1.32 \AA$ \\
\hline
\end{tabular}

Abbildung 2.1.: Perowskit-Struktur der Manganate (links) und Ionenradien (rechts). Gezeigt ist die kubische Einheitszelle von Verbindungen der Form $A B \mathrm{O}_{3}$, wo Platz $B$ mit tri- oder tetravalentem Mangan und $A$ mit trivalenten seltenen Erden oder divalenten Erdalkalimetallen besetzt ist. Um das kleine Mn-Ion bilden sechs Sauerstoff-Ionen ein Oktaeder. Gezeichnet mit VESTA MI08. Daten der Radien aus JV50. (leicht andere Werte auch zu finden in Sha76]).

erhalten Dag03, Kap. 2.4], sondern es findet eine Symmetrieerniedrigung durch kollektive Verkippung der $\mathrm{MnO}_{6}$-Oktaeder statt. Um diese Verzerrung zu quantifizieren, hat GoldsCHMid Tol26 einen Ausdruck eingeführt, der aus dem Abstand des Sauerstoff zum $A$-Platz $d_{A-\mathrm{O}}$ und zum $B$-Platz $d_{B-\mathrm{O}}$ den nach ihm benannten Goldschmidtschen Toleranzfaktor

$$
t=\frac{d_{A-\mathrm{O}}}{\sqrt{2} d_{B-\mathrm{O}}}=\frac{1}{\sqrt{2}} \frac{\left\langle r_{A}\right\rangle+r_{\mathrm{O}}}{\left\langle r_{B}\right\rangle+r_{\mathrm{O}}}
$$

errechnet, wobei sich die Abstände durch die Summen der Ionenradien bzw. der gemittelten Ionenradien ergeben. Demnach gilt für eine perfekt kubische Struktur $d_{A-\mathrm{O}}=\sqrt{2} d_{B-\mathrm{O}}$ und somit ist $t=1$. Wird der Faktor jedoch kleiner, verringert sich, wie schon angesprochen, durch die Verkippung der Oktaeder die Symmetrie und es entsteht eine Perowskit-Struktur, die man für $0.96<t<1$ als rhomboedrisch (R $\overline{3} \mathrm{c}$ ) und für $t<0.96$ als orthorhombisch (Pnma) bezeichnet [TT99]. Damit einher geht die Erniedrigung des Bindungswinkels $\mathrm{Mn}-\mathrm{O}-\mathrm{Mn}$, der dadurch kleiner als $180^{\circ}$ wird und nachhaltigen Einfluss auf die Mobilität der Elektronen und die magnetische Ordnung hat, wie sich später noch herausstellt.

Natürlich kann eine rein geometrische Betrachtung der Ionen die vielfältigen bei Manganaten auftretenden strukturellen Phänomene nicht erklären, denn ausgesprochen wichtig ist das Elektronensystem, hierbei im Besonderen die 3d-Elektronen des Manganatoms und die damit verbundenen Korrelationseffekte.

\subsubsection{Elektronische Struktur}

Ein neutrales Manganatom hat die elektronische Konfiguration von Argon und zusätzlich sieben weitere Elektronen, die sich auf $4 s$ - und $3 d$-Orbitale verteilen, konkret also $[\mathrm{Ar}] 4 s^{2} 3 d^{5}$. Nun kommt Mangan, wie bereits genannt, in den Oxidationsstufen +3 und 


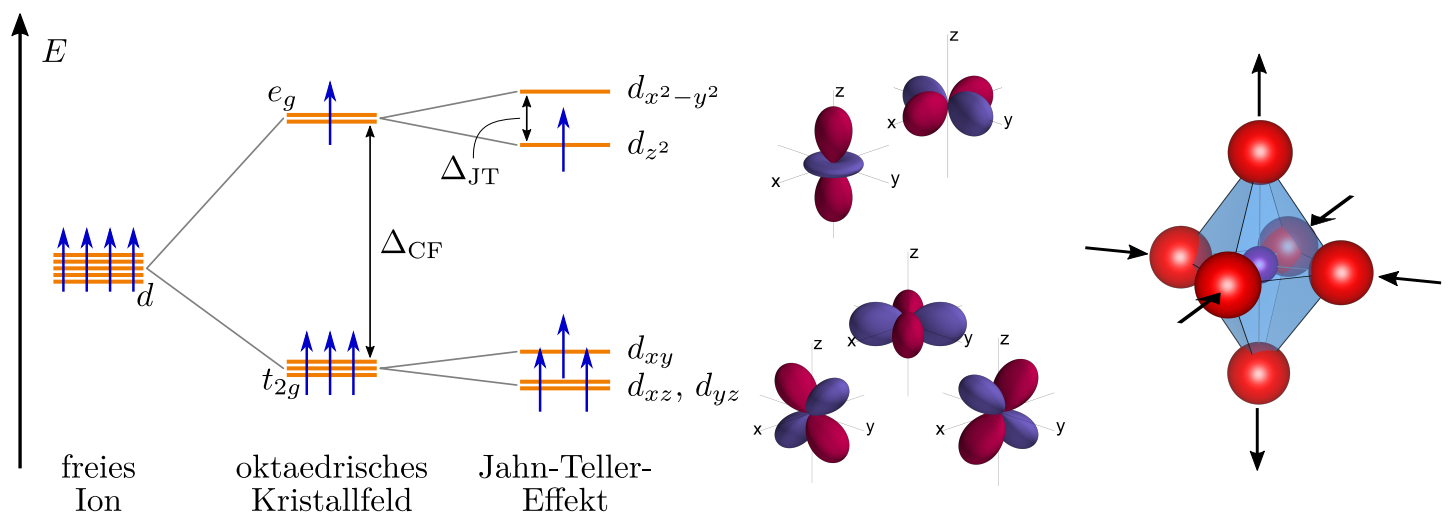

Abbildung 2.2.: Schema der Energieniveaus bei $\mathrm{Mn}^{3+}$ für die $3 d$-Elektronen mit Kristallfeldund Jahn-Teller-Aufspaltung (links) und gestrecktes Oktaeder (rechts). Von den vier Elektronen verteilen sich nach der Aufhebung der $d$-Orbital-Entartung drei auf das $t_{2 g^{-}}$und eins auf das $e_{g}$-Band, da die Hundsche Kopplungsenergie größer als die Kristallfeldaufspaltung $\Delta_{\mathrm{CF}}$ ist. Durch Elongation des $\mathrm{MnO}_{6}$-Oktaeders entlang der $z$-Achse spaltet sich $e_{g}$ erneut um die JahnTeller-Energie $E_{\mathrm{JT}}$ auf und die Energie für das $d_{z^{2}}$-Niveau kann gesenkt werden, sodass ein Nettogewinn entsteht. Nach Gro04, Kap. 4]. Orbitale mit mayavi [RV11] gezeichnet.

+4 vor, d. h. dreifach- bzw. vierfach ionisiert, sodass von den Valenzelektronen lediglich vier bzw. drei Elektronen im 3d-Orbital verbleiben. Wie auch Abb. 2.2 zeigt, betrachten wir jetzt zunächst den Fall mit $\mathrm{Mn}^{3+}$ : Dabei würden die vier $3 d$-Elektronen für ein freies Mn-Ion die entarteten $d$-Niveaus aufgrund der Hundschen Regeln mit parallelem Spin besetzen. Da jedoch das Ion in ein Kristallgitter eingebettet und dabei eben von einem Oktaeder aus sechs Sauerstoff-Ionen umringt ist, ergibt sich dadurch das so genannte Kristallfeld, in dem die Zustände in drei erniedrigte $t_{2 g^{-}}$und zwei erhöhte $e_{g}$-Niveaus aufspalten, die sich um die Kristallfeldenergie $\Delta_{\mathrm{CF}} \sim 1 \ldots 2 \mathrm{eV}$ Dag03, Kap. 4.2] unterscheiden. Eine anschauliche Erklärung dafür ist, dass die räumliche Orientierung der Orbitale $d_{z^{2}}{ }^{2}$ und $d_{x^{2}-y^{2}}$ auf den Sauerstoff gerichtet ist und es somit zur Coulombabstoßung mit seinen $p$-Orbitalen kommt, wohingegen $d_{x y}, d_{x z}$ und $d_{y z}$ von den Sauerstofforbitalen abgewandt sind. Dann besetzen die Elektronen, weil die Hundsche Kopplungsenergie $J_{\mathrm{H}} \sim 2 \ldots 3 \mathrm{eV}$ TT99 größer als $\Delta_{\mathrm{CF}}$ ist, wieder parallel orientiert die unteren drei und ein oberes Niveau und bilden dadurch einen High-SpinZustand mit $S=2$. Doch wie JAHN und Teller [JT37] ausführen, bleibt auch die Entartung von $t_{2 g}$ und $e_{g}$ nicht erhalten, sondern es gibt noch eine weitere Möglichkeit, die Gesamtenergie des Systems herabzusetzen, indem nämlich eine Modifikation der Struktur auftritt und das Oktaeder entlang der $z$-Achse gestreckt und in den anderen beiden Raumrichtung gestaucht wird. Dadurch werden nach derselben Argumentation wie zuvor $d_{z^{2}}, d_{x z}$ und $d_{y z}$ abgesenkt, hingegen $d_{x^{2}-y^{2}}$ und $d_{x y}$ angehoben, was aber dennoch leicht ersichtlich in der Summe zu einem Energiegewinn führt. Hierbei ist die

\footnotetext{
${ }^{2}$ Eigentlich müsste das Orbital korrekt mit $d_{3 z^{2}-r^{2}}$ bezeichnet werden, was aber häufig zu $d_{z^{2}}$ verkürzt wird.
} 
Aufspaltung der $e_{g}$-Orbitale von der Größenordnung $\Delta_{\mathrm{JT}} \sim 0.7 \mathrm{eV}$ Kov+10. Natürlich muss dafür elastische Energie im Kristallgitter aufgebracht werden, sodass sich ein Grundzustand in einem Energieminimum mit einer endlichen Verzerrung einstellt. Dieses Phänomen wird Jahn-Teller-Effekt genannt.

Nun kann man sich vorstellen, dass bei vielen Gitterplätzen mit $\mathrm{Mn}^{3+}$-Ionen diese Verzerrungen nicht unabhängig voneinander sein können und sich entsprechend kooperativ ausrichten müssen, sodass z. B. je eine gestauchte Ecke eines Oktaeders die gestreckte Ecke eines benachbarten bildet. Auf diese Weise kann die Kristallstruktur grundlegend beeinflusst werden und so findet man bei $\mathrm{LaMnO}_{3}$ (LMO) einen strukturellen Phasenübergang bei $\sim 780 \mathrm{~K}$ Mur+98 $\mathrm{I}^{3}$

Beim $\mathrm{Mn}^{4+}$ hingegen fehlt das vierte $3 d$-Elektron und es werden nur die $t_{2 g}$-Niveaus besetzt, sodass eine Jahn-Teller-Verzerrung keinen Energiegewinn bringen kann, da das $d_{z^{2}}$-Orbital frei ist. Folglich ist der Anteil an tetravalenten Mangan-Ionen maßgeblich für die Struktur verantwortlich, denn an den Gitterplätzen mit $\mathrm{Mn}^{4+}$ ist keine gemeinsame Verzerrung der Oktaeder möglich und mit zunehmender Dotierung, was bei LMO durch Strontium geschehen kann, verschiebt sich der Übergang zu tieferen Temperaturen Gro04, Kap. 4]. Genau dieses Zusammenspiel ist eine Hauptursache für die vielschichtige Physik der Manganate.

Nachdem nun auch die elektronische Struktur aufgezeigt wurde, sollen jetzt die grundlegenden Wechselwirkungen und die daraus resultierenden Eigenschaften dargestellt werden.

\subsubsection{Austauschmechanismen}

Für die magnetischen Phänomene ist es wichtig, zu wissen, was in den Manganaten die Ursache der magnetischen Momente sind. Dabei spielen die Sauerstoffionen keine Rolle, da sie in der Oxidationsstufe -2 in Edelgaskonfiguration vorliegen und daher alle Elektronenschalen abgeschlossen sind, sodass das daraus resultierende magnetische Gesamtmoment verschwindet. Ebenso gilt diese Argumentation auch für die Erdalkalimetallionen. Bei den höheren seltenen Erden jedoch spielen die stark lokalisierten $4 f$-Momente noch eine Rolle, was aber nicht für Lanthan gilt, denn dieses verfügt über keine $4 f$-Elektronen und trägt durch die Edelgaskonfiguration bei der Valenz +3 ebenfalls kein magnetisches Moment. In jedem Fall ist das Manganion in seinen zwei hier beschriebenen Oxidationsstufen Träger eines magnetischen Momentes, welches jedoch aufgrund des gequenchten Bahnmomentes nur vom Spin ausgeht [Gro04, Kap. 4]. Dieses ist, wie zuvor bereits diskutiert, aufgrund des High-Spin-Zustandes für $\mathrm{M}^{3+}$ durch den Gesamtspin $S=2$ und für $\mathrm{Mn}^{4+}$ durch $S=3 / 2$ gegeben.

${ }^{3}$ MuraKami et al. Mur+98 sehen den Übergang als orthorhombisch/orthorhombisch an, während frühere Messungen [WA59] orthorhombisch/rhomboedrisch angeben. Neuere Untersuchungen [MG03] sehen zwei strukturelle Übergänge: einen orthorhombisch-zu-orthorhombisch bei $\sim 750 \mathrm{~K}$ und einen orthorhombisch-zu-rhomboedrisch bei $\sim 1025 \mathrm{~K}$. 


\section{Superaustausch}

Zwischen den Mangan-Spins gibt es nun verschiedene Möglichkeiten der Wechselwirkung untereinander, von denen zunächst ein vereinfachtes System mit lediglich einem nichtentarteten, einfach besetzten Niveau betrachtet werden soll, das im so genannten Ein-Band-Hubbard-Modell beschrieben werden kann. Dafür kann man leicht einsehen, dass ein Austausch von Elektronen mit parallelem Spin zwischen zwei benachbarten Gitterplätzen aufgrund des Pauli-Prinzips verboten ist. Sind die Spins jedoch antiparallel orientiert, ist ein Transfer erlaubt und die Energie kann um

$$
\Delta E \propto-\frac{t^{2}}{U}
$$

abgesenkt werden, wobei $t$ das Matrixelement für das Hüpfen eines Elektrons zwischen zwei benachbarten Gitterplätzen ${ }^{4}$ und $U$ ein Maß für die Coulombabstoßung zwischen zwei Elektronen ist Mül88. Offensichtlich wird in diesem Modell eine antiparallele Ausrichtung benachbarter Spins energetisch favorisiert und damit kommt es effektiv zu einer antiferromagnetischen Kopplung.

Jedoch ist die tatsächliche Situation bei den Manganaten komplizierter, denn dort existieren mehrere entartete Niveaus, deren Entartung aber durch Kristallfeld und Jahn-Teller-Effekt aufgehoben ist, und der Austausch erfolgt nicht direkt zwischen den Mangan-Ionen, sondern aufgrund ihrer gegenseitigen Überlappung über die $2 p$ Orbitale des Sauerstoffs. Letztere sind um die Ladungstransferenergie $\Delta$ gegenüber den $e_{g}$-Niveaus ${ }^{5}$ abgesenkt und beeinflussen damit auch die Mobilität der Elektronen über virtuelle Hüpfprozesse (s. auch Abb. 2.3), die durch das Hüpf-Matrixelement zwischen den $d$-Orbitalen $t_{d d}=t_{p d}^{2} / \Delta$, welches natürlich auch von dem Überlapp zwischen den $p$ und $d$-Orbitalen und damit von dem Mn-O-Mn-Bindungswinkel abhängt, quantifiziert werden. Ebenso ist der Energiebeitrag $U$ für die Coulombabstoßung entscheidend, wobei typische Werte $t_{d d} \sim 0.1 \ldots 0.3 \mathrm{eV}$ und $U \sim 4 \ldots 7 \mathrm{eV}$ sind [BM14]. Für den Grenzfall $\Delta \gg U$ bevorzugen die virtuellen Hüpfprozesse die $\mathrm{O}_{2 p}$-Orbitale und die Elektronen werden lokalisiert, da auch $t_{d d}$ klein wird, sodass man einen Mott-Hubbard-Isolator erhält. Liegt hingegen das andere Extrem $U \gg \Delta$ vor, sind die $3 d$-Orbitale attraktiver und in den $2 p$-Orbitalen entstehen „Löcher“, was ebenfalls zur Lokalisierung der Elektronen führt und Landungstransfer-Isolator genannt wird. [ZSA85

Diese Hüpfprozesse haben jedoch, wie im vereinfachten Ein-Band-Hubbard-Modell schon gesehen, auch Konsequenzen für die magnetische Ordnung. So lassen sich nach den so genannten Goodenough-Kanamori-Anderson-Regeln (GKA-Regeln) Goo55. Kan59 And50 die magnetischen Wechselwirkungen ableiten, was schematisch in Abb. 2.3 gezeigt ist. Dafür spielen lediglich die itineranten $e_{g}$-Orbitale eine Rolle, sodass der Spin der stärker lokalisierten $t_{2 g}$-Orbitale zu $S=3 / 2$ zusammengefasst und durch die

\footnotetext{
${ }^{4}$ Streng genommen geht es um das Hüpfen zwischen zwei gleichen antibindenden Orbitalen auf benachbarten Gitterplätzen.

${ }^{5}$ Nur die $e_{g}$-Niveaus sind an dem Austausch beteiligt, da ihr Überlapp mit den $\mathrm{O}_{2 p}$-Orbitalen deutlich größer ist als der mit den $t_{2 g}$-Niveaus BM14.
} 
(a)

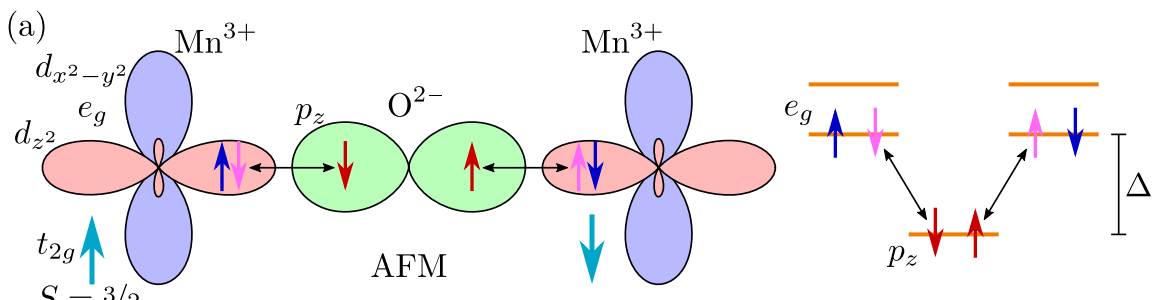

(b) $\mathrm{Mn}^{3+}$

AFM

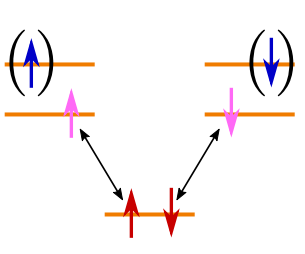

(c)
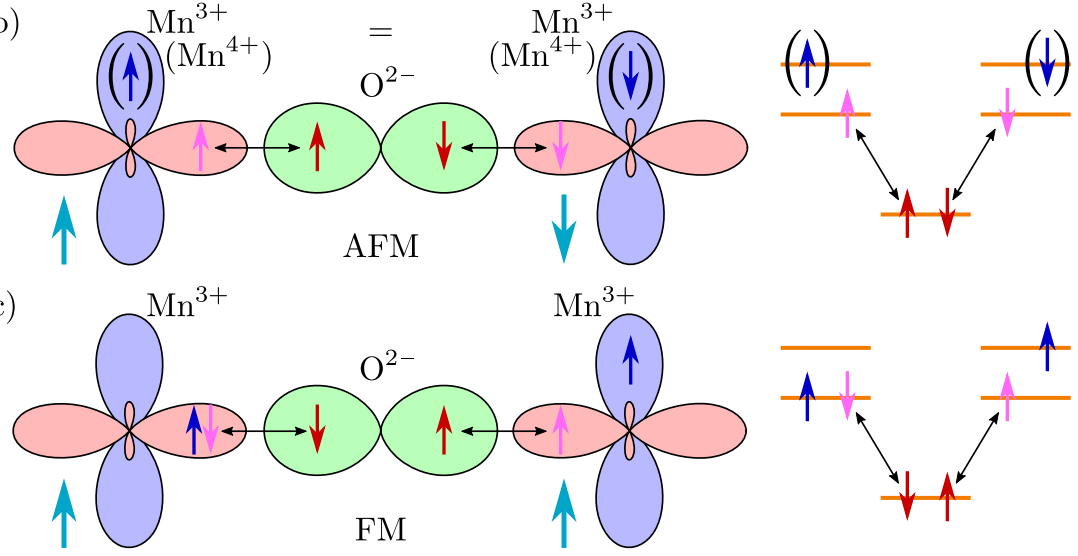

Abbildung 2.3.: Schema zur Veranschaulichung der GKA Regeln beim Superaustausch. Gezeigt ist der Austausch zwischen den $e_{g}$-Orbitalen isovalenter Mn-Ionen durch virtuelle Hüpfprozesse über die $p_{z}$-Orbitale des Sauerstoffs, welche um die Ladungstransferenergie $\Delta$ abgesenkt sind, für $180^{\circ}$-Bindungen. Die mit drei Elektronen besetzen $t_{2 g}$-Niveaus sind nicht direkt an der Wechselwirkung beteiligt, weswegen ihr Gesamtspin aufgrund der starken Hundschen Kopplung zu $S=3 / 2$ aufsummiert und parallel zum Spin des entscheidenden vierten Elektrons ist. (a) und (b): Zwischen besetzten und unbesetzten $d_{z^{2}}$-Orbitalen gibt es aufgrund von Pauliprinzip und Hundscher Kopplung eine antiferromagnetische Wechselwirkung. (c): Ist ein $d_{z^{2}}$ besetzt und das andere nicht, erfolgt eine leicht ferromagnetische Kopplung. Nach Gro04. Kap. 4] und BM14. 


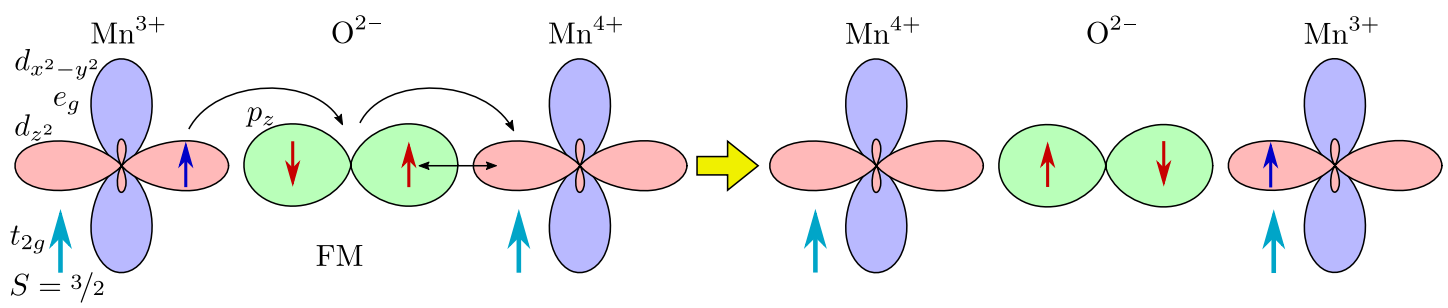

Abbildung 2.4.: Schema zur Veranschaulichung des Doppelaustauschs. Zwischen einem $\mathrm{Mn}^{3+}$ und einem $\mathrm{Mn}^{4+}$-Ion kann ein Aufelektron aus dem besetzten $d_{z^{2}}$-Orbital über das $p_{z^{-}}$-Orbital des $\mathrm{O}^{2-}$ in das unbesetzte entsprechende unbesetzte Orbital des benachbarten Ions quasi ohne Energiebarriere reell hüpfen. Die drei Elektronen in den $t_{2 g}$-Niveaus sind aufgrund der Hundschen Kopplung parallel orientiert sind hier nur zusammengefasst eingezeichnet. Dieser Austausch bevorzugt eine stark ferromagnetische Ordnung und delokalisiert die $e_{g}$-Elektronen, sodass der Zustand metallisch ist.

große Hundsche Kopplung parallel zu $e_{g}$ ausgerichtet ist. Hierbei sind für die virtuellen Hüpfprozesse über die Sauerstofforbitale auch geometrische Ausrichtungen entscheidend und so findet der Austausch nur über die $p_{z}$-Orbitale statt. Daraus ergibt sich für die Wechselwirkung zwischen gefüllten oder leeren $e_{g}$-Orbitalen aufgrund des Pauli-Prinzips und der großen Hundschen Austauschenergie eine stark antiferromagnetische Kopplung Gro04, Kap. 4], was die Fälle (a) und (b) in Abb. 2.3 zeigen. Hingegen lässt der Austausch zwischen besetzten und unbesetzten $e_{g}$-Niveaus wie in Fall (c) nach selben Prinzipien wie eben genannt die Momente schwach ferromagnetisch koppeln 6

\section{Doppelaustausch}

Treten nun gemischte Valenzen des Mangans, also $\mathrm{Mn}^{3+}$ und $\mathrm{Mn}^{4+}$ auf, so ist bei letzterem keines der $e_{g}$-Niveaus besetzt und die Wechselwirkung, die von ZENER Zen51] Doppelaustausch (engl. Double Exchange) genannt wurde, gestaltet sich anders. Denn, wie in Abb. 2.4 veranschaulicht, kann aus dem besetzten $d_{z^{2}}$ Orbital des $\mathrm{Mn}^{3+}$ z. B. ein Aufelektron in das $\mathrm{O}_{p_{z}}$-Orbital hüpfen, während dann natürlich gleichzeitig auch ein Aufelektron in das entsprechende $3 d$-Orbital des benachbarten $\mathrm{Mn}^{4+}$ wechselt, sodass effektiv $\mathrm{Mn}^{3+}$ und $\mathrm{Mn}^{4+}$ die Plätze tauschen. Weiterhin richten sich wieder die jeweiligen Spins der lokalisierten $t_{2 g}$-Niveaus aufgrund der Hundschen Kopplung parallel aus und so ergibt sich dann insgesamt eine stark ferromagnetische Kopplung. Dabei sind diese Hüpfprozesse reell und nicht wie beim Superaustausch virtuell, da sie energieneutral verlaufen, wodurch sich ein metallischer Zustand einstellt, wohingegen der Superaustausch die Lokalisation der Elektronen und damit einen isolierenden Zustand favorisiert. Die Dotierung eines Manganats wie z. B. LMO mit Strontium fügt $\mathrm{Mn}^{4+}-$ Valenzen ein und führt folglich zu ferromagnetisch metallischem Verhalten. Gro04, Kap. 4; BM14 Außerdem sei noch bemerkt, dass auch der Winkel $\theta$ zwischen den

\footnotetext{
${ }^{6}$ Es gibt noch eine weitere GKA Regel zum Austausch über $90^{\circ}$-Bindungen, der hier nicht dargestellt ist, da bei den pseudokubischen Manganaten die Bindungswinkel nahe $180^{\circ}$ sind und er daher in erster Näherung vernachlässigt werden kann BM14.
} 
$t_{2 g}$-Rumpfspins bedeutsam ist, da die Stärke des Doppelaustauschs mit $\cos (\theta / 2)$ skaliert, was auch zu so genannten verkanteten (engl. canted) Zuständen führt BM14, Gen60: 7 Damit bietet der Doppelaustausch auch eine qualitative Erklärung für den CMR Effekt, da sich ja dort ferromagnetische Ordnung und elektrische Leitfähigkeit bedingen.

Zusammenfassend lässt sich also feststellen, dass für die elektrische Leitfähigkeit und magnetischen Eigenschaften das Verhältnis von $\mathrm{Mn}^{3+} \mathrm{zu} \mathrm{Mn}^{4+}$ ebenso wie der davon nicht unabhängige Mn-O-Mn-Bindungswinkel entscheidend sind, weil sie die vorherrschenden Austauschmechanismen bestimmen, welche dann für Lokalisation mit isolierendem (Superaustausch) oder eben Delokalisation (Doppelaustausch) der Elektronen und die magnetische Kopplung (Superaustausch: vorherrschend AFM, Doppelaustausch: FM) sorgen. So ergibt sich dadurch für viele dotierte Manganatverbindungen ein reichhaltiges Phasendiagramm mit verschiedenen magnetischen und elektronischen Phasen.

\subsubsection{Polaronen}

Wie zuvor gesehen, hat die Besetzung des $e_{g}$-Orbitals im Mangan durch ein Elektron weitreichende Folgen für die Gitterstruktur, was sich in der Jahn-Teller-Verzerrung widerspiegelt. Und diese hat umgekehrt ebenfalls starken Einfluss auf die Mobilität der Elektronen, da sie den Mn-O-Mn-Bindungswinkel und damit den Überlapp der Orbitale ändert. Damit tritt hier ein Phänomen auf, das allgemein aus der Festkörperphysik bekannt ist und zwar, dass sich ein Elektron in einem Festkörper nicht unabhängig von seiner Umgebung bewegen kann, sondern von einer selbst hervorgerufenen strukturellen oder magnetischen Modifikation umringt ist, die es mitführen muss. Dabei lässt sich diese Betrachtung natürlich auch auf andere Ladungsträger wie „Löcher ‘8 übertragen. Diesem Ladungsträger inklusive seiner ihn „kleidenden“ Veränderung schreibt man unter Anwendung eines bekannten Konzepts aus der Festkörperphysik den Charakter eines Quasiteilchens namens Polaron zu, dessen Mobilität natürlich im Vergleich zum freien Ladungsträger stark eingeschränkt ist. Davon sind einige verschiedene Arten in Abb. 2.5 schematisch dargestellt. So ist leicht einsehbar, dass bei dem Vorhandensein eines $\mathrm{Mn}^{4+}$-Ions aufgrund des kleineren Ionenradius gegenüber einem $\mathrm{Mn}^{3+}$ das umgebende Sauerstoffoktaeder näher an das Ion heranrücken kann (a), wobei ein dielektrisches Polaron entsteht. Sortieren sich die $d_{z^{2}}$-Orbitale um ein tetravalentes Mangan zur Maximierung des Doppelaustauschs, falls dieser dominierend ist (c), handelt es sich um ein orbitales Polaron. Weiter können aber auch die Spineigenschaften der Elektronen wichtig werden, da sich durch den Doppelaustausch über das $e_{g}$-Elektron die aufgrund der Hundschen Wechselwirkung daran parallel gekoppelten $t_{2 g}$-Momente ferromagnetisch ausrichten und so an das $e_{g}$-Elektron binden (d), was man daher Spinpolaron nennt. Zuletzt sei noch der eingangs in diesem Abschnitt erwähnte, wichtige Fall (b) erklärt, denn dieser hat laut MiLLis et al. MLS95 bei den Manganaten neben dem Doppelaustausch

\footnotetext{
${ }^{7}$ In der Literatur ist die Existenz dieser Zustände allerdings umstritten, denn es könnte sich auch z. B. um in eine antiferromagnetische Matrix eingebettete, isolierte ferromagnetische Teilbereiche handeln.

${ }^{8}$ oder Protonen oder Myonen
} 


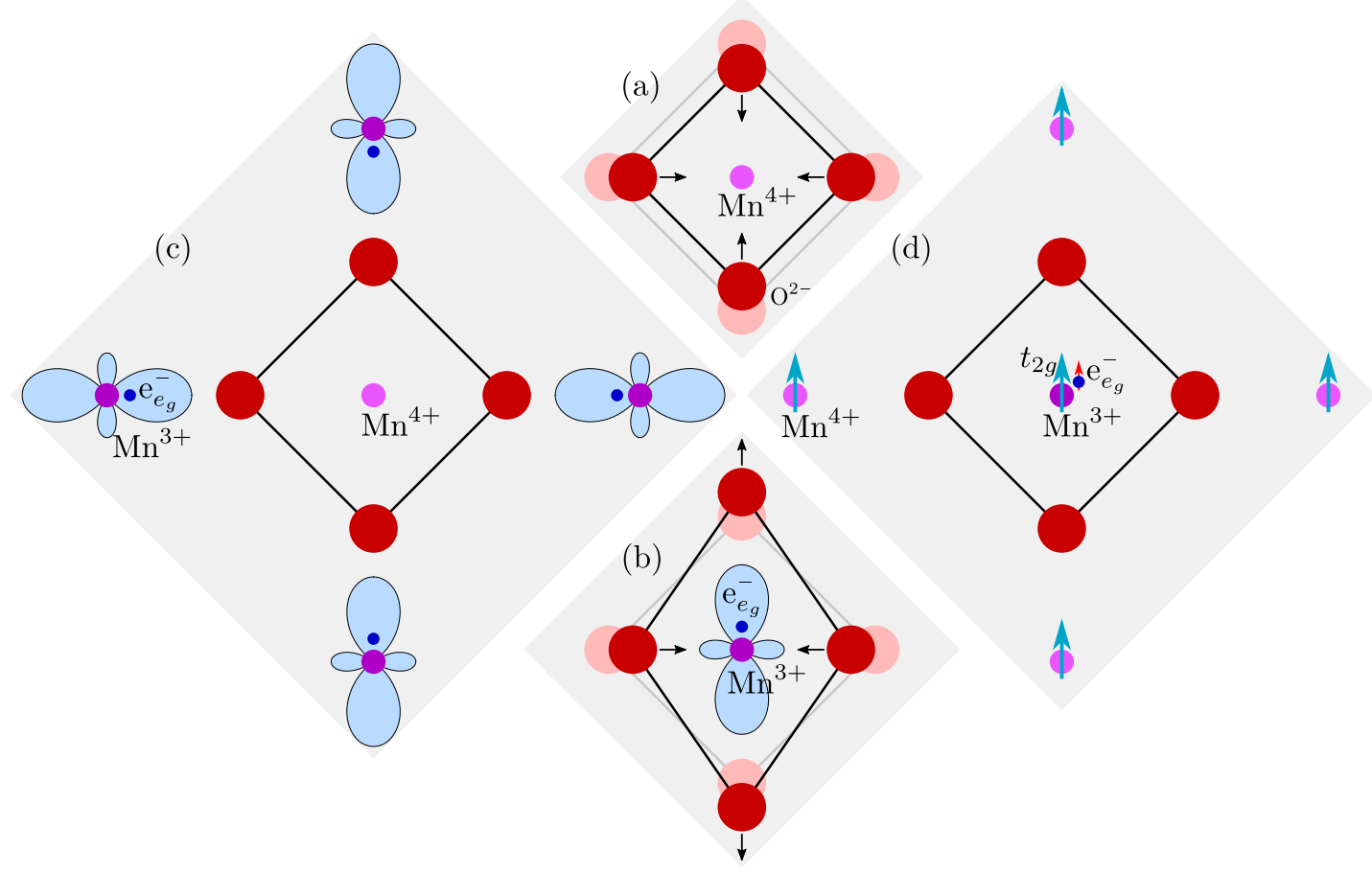

Abbildung 2.5.: Schemata verschiedener Polaronen. Gezeigt sind jeweils ein zentrales und ggf. benachbarte Manganionen in einem zweidimensionalen Schnitt durch das Sauerstoff-Oktaeder. In jedem der vier gezeigten Fälle ruft das Elektron oder „Loch“ eine Änderung der es umgebenden Struktur (Gitter, Orbitale, Spinausrichtung) hervor und ist an diese gebunden. Folglich muss es bei einer Bewegung diese „kleidende“ Modifikation mitziehen, was die Mobilität einschränkt. Die hier dargestellten Fälle sind: (a) Dielektrisches Polaron: Bei einem $\mathrm{Mn}^{4+}$-Ion können aufgrund des verkleinerten Ionenradius die umliegenden Sauerstoffionen näher zusammenrücken. (b) JahnTeller-Polaron: Durch das $e_{g}$-Elektron im $d_{z^{2}}$-Orbital senkt eine Jahn-Teller-Verzerrung des Oktaeders die Energie. (c) Orbitales Polaron: Ist der Doppelaustausch energetisch bevorzugt, kann dies zu einer kollektiven Ausrichtung benachbarter $d_{z^{2}}$-Orbitale auf das $\mathrm{Mn}^{4+}$-Ion führen . (d) Spinpolaron: Auch die ferromagnetische Kopplung des Doppelaustauschs kann das $t_{2 g}$-SpinMoment von benachbarten $\mathrm{Mn}^{4+}$-Ionen an ein $e_{g}$-Elektron binden. Für (a) und (c) hat das Polaron „Loch“- für (b) und (d) Elektron-Charakter. Nach Gro04, Kap. 4] 


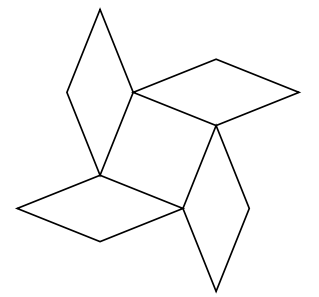

Abbildung 2.6.: Stark vereinfachtes Schema zur Veranschaulichung korrelierter Polaronen. Die Stauchung einer Oktaeder-Diagonalen bildet die Elongation der Diagonalen eines benachbarten Oktaeders. Gezeigt ist nur die Ausrichtung in einer Ebene.

den größten Einfluss auf die Mobilität der Ladungsträger ${ }^{9}$. Dort ruft ein Elektron im $d_{z^{2}}$-Orbital eines $\mathrm{Mn}^{3+}$-Ions, wie man bereits in Kap. 2.1.2 gesehen hat, eine Verzerrung des umgebenden Sauerstoff-Oktaeders hervor, da sich dadurch die Coulombabstoßung verringern lässt. Aufgrund des verursachenden Effektes nennt man das Quasiteilchen auch entsprechend Jahn-Teller-Polaron. Dabei liegt der typische Energiegewinn speziell für $\mathrm{La}_{0.85} \mathrm{Sr}_{0.15} \mathrm{MnO}_{3}$ nach Rechnungen bei $\Delta E_{\mathrm{JT}} \sim 0.6 \mathrm{eV}$ pro Polaron, mindestens aber $\Delta E_{\mathrm{JT}} \gtrsim 0.1 \mathrm{eV}$ Mil96.

Natürlich kann es dann auch zu Wechselwirkungen zwischen die Polaronen kommen, sodass z. B. zwei Polaronen ein Paar zu einem stark lokalisierten Bipolaron bilden [AB99] oder mehrere Polaronen können sich in einem korrelierten Zustand zusammenschließen. Dafür sei noch einmal daran erinnert, dass ja die Jahn-Teller-Verzerrungen hervorragend dazu geeignet sind, kooperativ aufzutreten, denn eine verkürzte Diagonale des Sauerstoffoktaeders in einer Raumrichtung kann für die andere Raumrichtung eines benachbarten Oktaeders gleichzeitig die Verlängerung der Diagonalen darstellen. Daraus ergibt sich, wie Abb. 2.6 veranschaulicht, ein regelmäßiges Gefüge, was als korrelierte Polaronen bezeichnet wird [Nel+01]. Für deren Ausdehnung finden NELSON et al. [Nel+01] bei $\mathrm{La}_{0.7} \mathrm{Ca}_{0.3} \mathrm{MnO}_{3}(\overline{\mathrm{LCMO}})$ und $\mathrm{Pr}_{0.7} \mathrm{Ca}_{0.3} \mathrm{MnO}_{3}$ (PCMO) mit Röntgen- und Neutronenstreuung Korrelationslängen von 1...2 Gitterkonstanten bei höheren Temperaturen, am Übergang jedoch nimmt das Streusignal der Polaronen zu. Nun haben diese beiden Systeme jedoch aufgrund der unterschiedlichen Ionenradien von Lanthan und Praseodym (s. Abb. 2.1) verschieden große Elektron-Phonon-Kopplung, welche das Verhalten der Polaronen wesentlich beeinflusst. Daher verschwindet die Signatur der Polaronen für LCMO nach dem Übergang in die ferromagnetisch-metallische Phase, wohingegen für PCMO beim Übergang in die Ladungsordnung die Korrelationslänge eine Größe von $\sim 170 \AA$ und damit einige zehn Gitterkonstanten annimmt. Die Korrelationen spielen also eine wichtige Rolle bei den Phasenübergängen der Manganate Kir+03, Lou+97].

\subsubsection{Phasendiagramme}

Da die Elektron-Phonon-Kopplung die Ausprägung der Korrelationen und damit der korrelierten Polaronen bestimmt, ist es sinnvoll, ein generisches Phasendiagramm der Manganate in Abb. $2.7 \mathrm{zu}$ betrachten. Im Wesentlichen sind dort drei Bereiche zu sehen: Für hohe Temperaturen ergibt sich eine paramagnetische Phase, die durch die Lokalisation der Ladungsträger isolierenden Charakter besitzt. Jetzt geht bei großer

\footnotetext{
${ }^{9}$ Der Doppelaustausch delokalisiert und Polaronen lokalisieren Elektronen.
} 

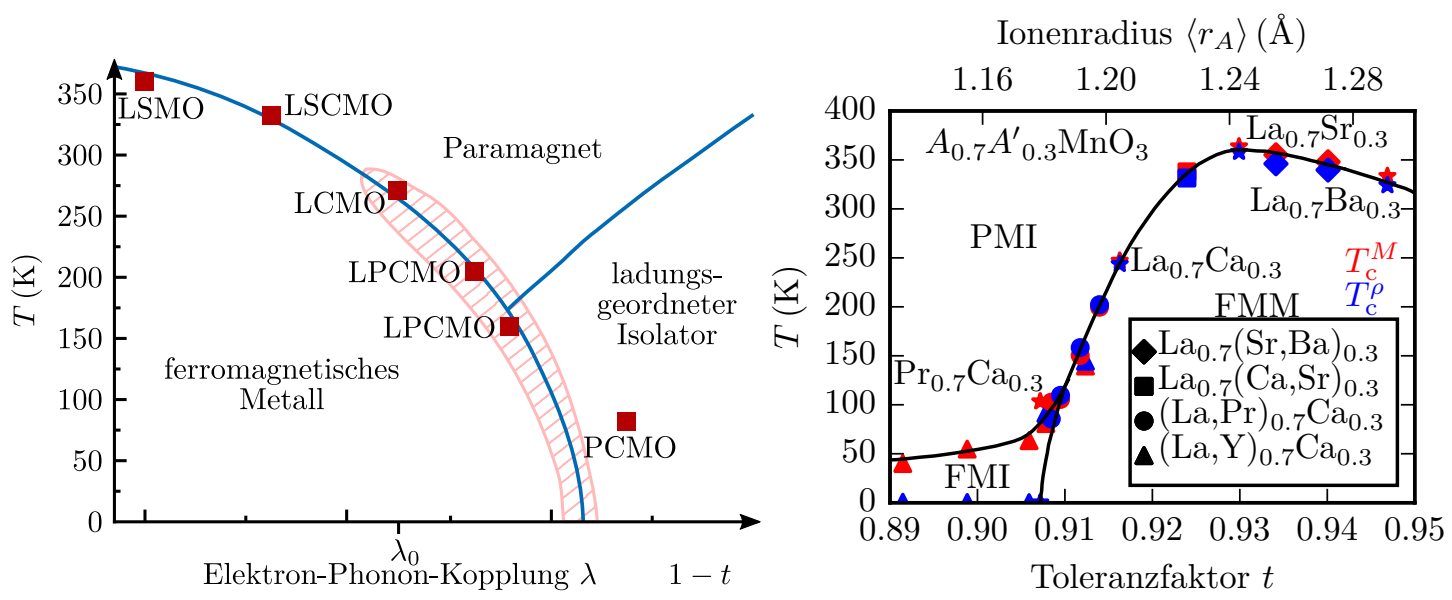

Abbildung 2.7.: Generisches Phasendiagramm der Manganate. Im linken Diagramm haben MoshnYaGa und Samwer [MS11] die Abbildung aus [ML03] (hier nicht gezeigt) mit den Daten der optimal dotierten Manganate (rote Quadrate) aus Hwa+95] (hier rechts) ergänzt. Dabei verhält sich der Toleranzfaktor $t$ qualitativ umgekehrt zur Elektron-Phonon-Kopplung $\lambda$, d. h. $\lambda \propto(1-t)$. Der schraffierte Bereich kennzeichnet die Phasengrenze, bei welcher der Übergang von erster Ordnung ist, also mesoskopische Phasenkoexistenz vorliegt. LBMO ist links nicht eingezeichnet. Es hat aufgrund des großen Ba-Ionenradius einen großen Toleranzfaktor und müsste sich damit eigentlich links von LSMO mit sehr kleinem $\lambda$ befinden (siehe Fließtext).

Elektron-Phonon-Kopplung $\lambda$, wie für PCMO, diese in eine vollständig ladungsgeordnete, isolierende Phase über. Dem gegenüber ist bei kleinerem $\lambda$, wie LPCMO, der Grundzustand ferromagnetisch und metallisch. Dabei sind die Phasenübergänge für $\lambda<\lambda_{0}$ kontinuierlich und damit von zweiter Ordnung. Doch in dem schraffierten Bereich findet man Übergänge erster Ordnung, die sich durch Phasenkoexistenz - d. h. separierte Bereiche aus isolierender und metallischer Phase - auszeichnen, von der angenommen wird, dass sie entscheidend für den CMR-Effekt ist Ueh+99, DHM01.

Nun stellt sich noch die Frage, wo das für diese Arbeit wichtige System LBMO eingeordnet werden kann. Im rechten Diagramm von Abb. 2.7 ist es mit größerem Toleranzfaktor als LSMO eingezeichnet, da Barium den größeren Ionenradius besitzt. Folglich sollte es im linken Bild auch links von LSMO platziert werden, was aber gleichzeitig bedeuten würde, dass die Elektron-Phonon-Wechselwirkung schwächer sein müsste. Jedoch ist die Übergangstemperatur von LBMO niedriger als die von LSMO, trotz des größeren Toleranzfaktors. Dieses begründen Rodriguez-MARTinez und ATTField RA96 nach Röntgendiffraktometrie-Messungen an Bulk-Proben durch eine größere Unordnung im Gitter aufgrund der zufällig verteilten Kationen mit stark unterschiedlichen Ionenradien. Was dieses jedoch für die Elektron-Phonon-Kopplung bedeutet, bleibt offen, denn aus der Betrachtung des Toleranzfaktors müsste $\lambda$ sehr klein sein, aber die Übergangstemperatur legt einen größeren Wert nahe. 


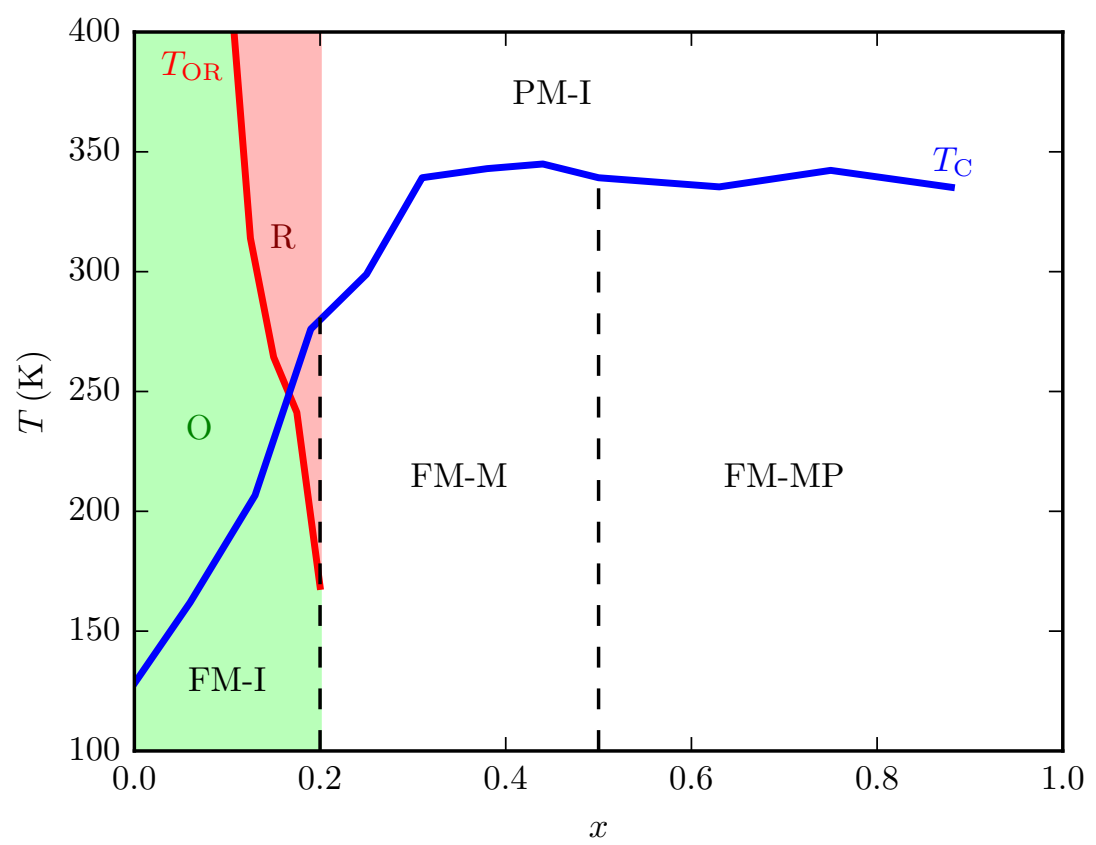

Abbildung 2.8.: Phasendiagramm von $\mathrm{La}_{1-x} \mathrm{Ba}_{x} \mathrm{MnO}_{3}$. Die Hochtemperaturphase ist immer paramagnetisch-isolierend (PM-I) und die Tieftemperaturphase immer ferromagnetisch (FM) ordnend. Für kleine Dotierungen stellt sich bei tiefen Temperaturen isolierendes (FM-I), im mittleren Bereich metallisches (FM-M) Verhalten ein. Ein struktureller Phasenübergang existiert bei niedrigen Dotierungen von orthorhombisch $(\mathrm{O})$ nach rhomboedrisch $(\mathrm{R})$. Bei $x>0.5$ wird eine Mischphase (FM-MP) gefunden, die evtl. aufgrund einer chemischen Entmischung bei der

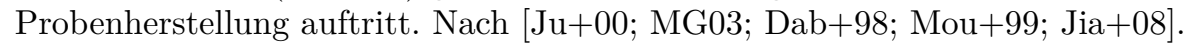

\section{Lanthan-Barium-Manganat}

Das System $\mathrm{La}_{1-x} \mathrm{Ba}_{x} \mathrm{MnO}_{3}$ (LBMO) ist eine der prototypischen CMR Verbindungen, wobei es durch stark unterschiedliche Ionenradien ausgezeichnet ist, da Barium das größte der gängigen $A$-Kationen ist (s. Abb. 2.1). In der Verbindung tritt Lanthan trivalent und Barium divalent auf, wodurch die Dotierung $x$ gleichzeitig als Lochdotierung den Anteil an $\mathrm{Mn}^{4+}$-Ionen angibt und damit durch den Doppelaustausch ferromagnetische Ordnung begünstigt. Zudem ist bei geeignetem Ba-Anteil der Übergang auf Raumtemperatur einstellbar, was das System interessant macht für Messaufbauten, die nicht über extensive Temperaturkontrolle verfügen.

Das Phasendiagramm, welches in Abb. 2.8 gezeigt ist, haben $\mathrm{JU}$ et al. $\mathrm{Ju}+00$ ] mithilfe von feld- und temperaturabhängigen Messungen der Magnetisierung, temperaturabhängigen Messungen des Widerstandes und Röntgendiffraktometrie-Messungen an Bulk-Proben erstellt, die durch Schmelzreaktionen hergestellt wurden. Dabei fanden sie immer für die Hochtemperaturphase paramagnetisch-isolierendes Verhalten und im Grundzustand eine ferromagnetische Ordnung, die für Dotierungen zwischen 
$0.13<x<0.5$ das theoretisch zu erwartende maximale magnetische Moment 10 zeigten, darüber oder darunter jedoch die Sättigungsmagnetisierung schnell abnimmt. Dieses ist nachvollziehbar, da sich nur mit ausreichender Lochdotierung ein ferromagnetischer Zustand durch den Doppelaustausch einstellen kann bzw. bei zu hoher Dotierung die antiferromagnetische Kopplung des Superaustauschs zwischen den $\mathrm{Mn}^{4+}$-Ionen und auch die zusätzliche Unordnung im Gitter entgegenwirkt.

Beim elektrischen Transport ergeben sich jedoch andere Phasen unterhalb des Übergangs: Während sich für schwache Dotierungen $(x<0.2)$ aufgrund der Ladungsordnung ein isolierendes Verhalten zeigt, ist im mittleren Dotierungsbereich $0.2<x<0.5$ das System metallisch. Bei größeren Dotierungen werden die Proben zunehmend isolierend, was die Autoren mit einer magnetischen Mischphase begründen, bei der isolierte ferromagnetische Domänen in eine antiferromagnetische Matrix eingebettet seien. Tatsächlich sollten die Proben auch ohne die Begründung durch die Mischphase bei höheren Dotierungen isolieren, da auch hier die Ladungsordnung für eine Lokalisation der Ladungsträger sorgt. In jedem Fall könnte die Mischphase auch durch chemische Entmischung bei der Probenherstellung entstehen, um die zunehmende Verzerrung der Struktur aufgrund der stark unterschiedlich großen Ionenradien von La und Ba zu vermeiden.

Zusätzlich lassen sich aus den Messungen von Mandal und GHosh MG03 an gezogenen Einkristallen Angaben zu den strukturellen Eigenschaften gewinnen. So gibt es für $x<0.2$ einen strukturellen Phasenübergang, dessen Tieftemperaturphase orthorhombisch und Hochtemperaturphase rhomboedrisch ist, wobei die Übergangstemperatur $T_{\mathrm{OR}}$ bei $x=0$, was $\mathrm{LMO}$ entspricht, über $1000 \mathrm{~K}$ liegt ${ }^{11}$ und mit höherer Dotierung stark abfällt. Ab $x>0.2$ könnte $T_{\mathrm{OR}}$ wenn überhaupt nur noch sehr klein sein, jedoch findet man in der Literatur keine Untersuchungen, die einen strukturellen Phasenübergang bei diesen Dotierungen nahelegen.

Abschließend sollen noch die korrelierten Polaronen bei LBMO diskutiert werden, was schwierig ist, da sich das System, wie zuvor schon erklärt, nicht einfach in das generische Phasendiagramm (Abb. 2.7, links) einordnen lässt. Je nachdem, ob es sich dort links oder rechts von LSMO befindet, sind durch Elektron-Phonon-Kopplung korrelierte Polaronen oder eben keine zu erwarten. Für Letzteres sprechen die Untersuchungen von Chen et al. [Che+08], die mithilfe von Neutronenstreuung durchaus Polaronen in LBMO fanden, ihnen aber rein dynamischen, isolierten Charakter und keine Wechselwirkung über Phononen zusprachen. Jedoch fanden diese Analysen an Einkristallen [Bar+00 statt und in dünnen Filmen können zusätzliche Verspannungen auftreten, welche die Elektron-Phonon-Wechselwirkung verstärken und damit die Bildung korrelierter Polaronen begünstigen.

\section{Lanthan-Praseodym-Calcium-Manganat}

Ein weiteres prototypisches System ist $\left(\mathrm{La}_{1-y} \mathrm{Pr}_{y}\right)_{1-x} \mathrm{Ca}_{x} \mathrm{MnO}_{3}$ (LPCMO), welches das divalente Calcium und die trivalenten Ionen Lanthan und Praseodym enthält. Dabei

\footnotetext{
${ }^{10} m=g \mu_{\mathrm{B}}\langle S\rangle$ mit $g=2$ und $\langle S\rangle=(1-x) \cdot 2+x \cdot 3 / 2$, weil ja $S\left(\mathrm{Mn}^{3+}\right)=2$ und $S\left(\mathrm{Mn}^{4+}\right)=3 / 2$

${ }^{11}$ Siehe auch Fußnote 3 auf Seite 8
} 


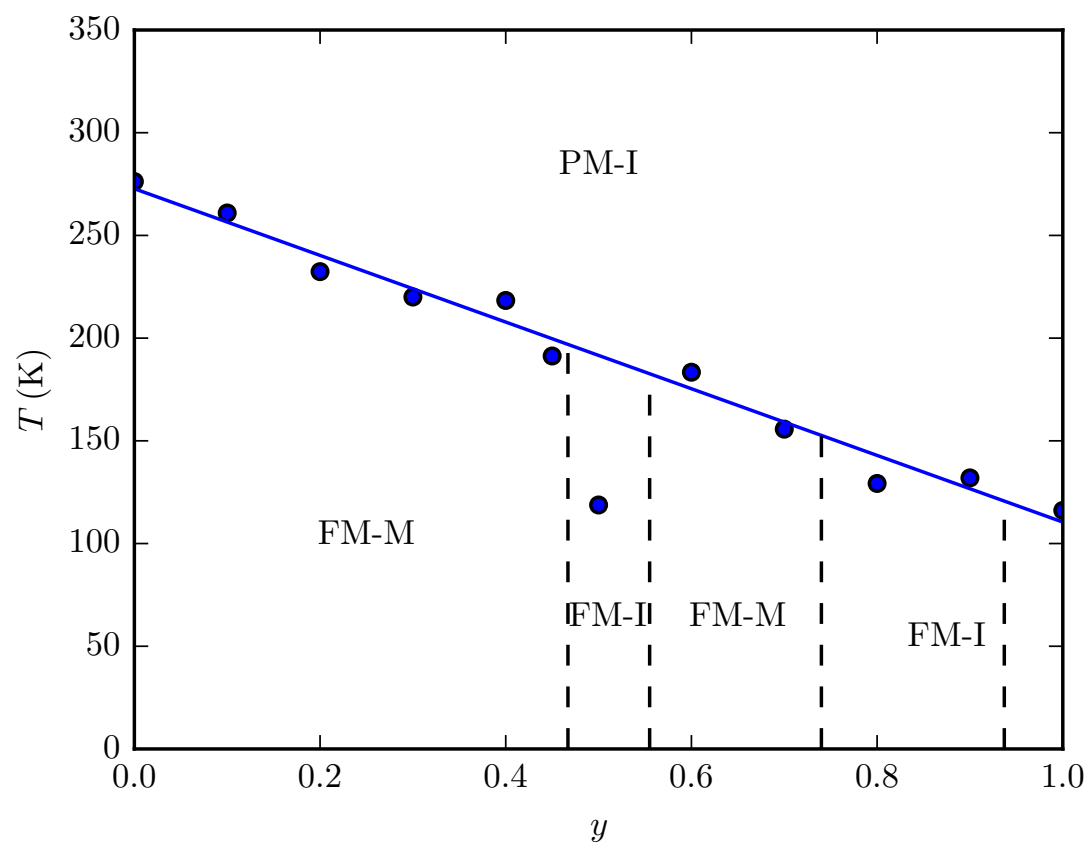

Abbildung 2.9.: Phasendiagramm von $\left(\mathrm{La}_{1-y} \mathrm{Pr}_{y}\right)_{0.67} \mathrm{Ca}_{0.33} \mathrm{MnO}_{3}$. Die Hochtemperaturphase ist paramagnetisch-isolierend (PM-I), ansonsten stellt sich ein aufgrund des Doppelaustauschs zu erwartende ferromagnetische Ordnung als Grundzustand ein. Generell ist das System für $y<0.75$ (Perkolationsgrenze) metallisch und darüber isolierend. Jedoch fällt die Dotierung $y=0.5$ aus der Reihe, was durch Kationen- und Ladungsordnung erklärt werden könne. Nach Hüh10.

eignet es sich bei konstanter Dotierung $x$, die ja den Anteil an $\mathrm{Mn}^{4+}$-Ionen angibt, besonders dafür, nur den Einfluss unterschiedlich großer Ionenradien, was durch La und Pr gegeben ist (s. Abb. 2.1), bei konstanter Manganvalenz zu studieren. Dieses taten Uehara et al. [Ueh+99] und sind dort auf die elektronische Phasenseparation ${ }^{12}$ in der Nähe des Metall-Isolator-Übergangs gestoßen, bei der ferromagnetisch-metallische und ladungsgeordnete isolierende Bereiche mit einer Ausdehnung von einigen hundert Nanometern in der Probe koexistieren [Bec+02]. Also wird der metallische Transport über Perkolationspfade durch die metallischen Bereiche realisiert May+01.

Von diesem Manganat $\left(\mathrm{La}_{1-y} \mathrm{Pr}_{y}\right)_{1-x} \mathrm{Ca}_{x} \mathrm{MnO}_{3}$ (LPCMO) mit $x \sim 0.33$, also in der Form $\left(\mathrm{La}_{1-y} \mathrm{Pr}_{y}\right)_{0.67} \mathrm{Ca}_{0.33} \mathrm{MnO}_{3}$, hat nun HüHN Hüh10 im Rahmen seiner Diplomarbeit dünne Filme präpariert, wobei die Schichten mithilfe der MAD-Technik auf MgO-Substraten mit der Orientierung (100) hergestellt wurden. Anschließend konnte er aus Röntgendiffraktometrie-, Widerstands- und Magnetisierungsmessungen ein detailliertes Phasendiagramm erstellen, welches Abb. 2.9 zeigt. Auch bei diesem System ist wie

\footnotetext{
${ }^{12}$ Der Begriff „Phase“ ist hier nicht im thermodynamischen Sinn zu verstehen, sondern beschreibt den Umstand, dass man lokal eine elektronische und Gitterkonfiguration findet, welche das Material sonst nur unter anderen Bedingungen, dann aber global als tatsächliche thermodynamische Phase ausbildet. Dag03, Kap. 1.3]
} 
bei LBMO die Hochtemperaturphase paramagnetisch-isolierend und der Grundzustand ordnet über den gesamten Dotierungsbereich aufgrund des Doppelaustauschs ferromagnetisch. Elektrisch betrachtet gibt es im Wesentlichen eine metallische für niedrige und eine isolierende Tieftemperaturphase für hohe Dotierungen, die durch die Perkolationsgrenze bei $y \sim 0.75$ getrennt sind. Jedoch ergibt sich für $y=0.5$ eine Besonderheit, die $T_{\mathrm{C}}$ stark zu tiefen Temperaturen verschiebt und den Grundzustand isolierend werden lässt. HüHN [Ḧ̈h10 schließt chemische Entmischung zu LCMO und PCMO aus, da in diesem Fall die Magnetisierung der Probe höher sein müsste, was aber nicht der Fall war. Aufgrund der besonderen Stöchiometrie $\mathrm{La}_{0.33} \mathrm{Pr}_{0.33} \mathrm{Ca}_{0.33} \mathrm{MnO}_{3}$ schlägt er daher eine Kationenund daraus resultierende Ladungsordnung als Begründung vor.

Strukturell sollte LPCMO laut einfacher Berechnung des Strukturfaktors bei $y<0.85$ rhomboedrisch sein und erst für hohe Dotierungen orthorhombisch werden [Hüh10]. Jedoch wird die Struktur von noch weiteren Faktoren wie zum Beispiel durch die ElektronPhonon-Kopplung beeinflusst, die ja über Jahn-Teller-Verzerrungen zur Rotation der Oktaeder und damit zur Reduzierung der Symmetrie führt. Und da in diesem System die Elektron-Phonon-Wechselwirkung stark ist, liegt über den gesamten Dotierungsbereich $y$ eine orthorhombische Phase vor [Hüh10]. Somit sollte LPCMO deutlich mehr korrelierte Polaronen aufweisen als LBMO,

\subsection{Dritte harmonische Spannung}

Zur Untersuchung der Korrelationen, also der korrelierten Polaronen, in den Manganaten wird eine Messmethode benötigt, welche auf diese direkt oder indirekt sensitiv ist. Da nun die korrelierten Polaronen mit einer lokalen Strukturänderung im Gitter einhergehen, ist es naheliegend, diese direkt durch Abbildung der Gittersymmetrie mittels Röntgendiffraktometrie, Neutronenstreuexperimenten [Che+08] oder Transmissionselektronenmikroskopie (TEM) nachzuweisen.

Hier soll allerdings ein anderes indirektes Verfahren beschrieben werden, welches über die Messung der dritten harmonischen Spannung, auch $3 \omega$-Messung genannt ${ }^{13}$ Zugriff auf die Korrelationen bekommt. So stellten Moshnyaga et al. Mos+09 ${ }^{14}$ bei elektrischen Vierpunktmessung mit Wechselfeld 15 an Manganaten fest, dass für Systeme mit korrelierten Polaronen wie LCMO eine starke Erhöhung der dritten harmonischen Spannung $U_{3 \omega}$ in der Nähe des Metall-Isolator-Übergangs auftritt, wohingegen bei Systemen, wo diese Korrelationen fehlen, kein erhöhtes Signal messbar war. Dabei untersuchten sie speziell das Verhältnis aus der auf der dritten harmonischen Frequenz $3 \omega$ gemessenen Spannung $U_{3 \omega}$ und der auf der fundamentalen Frequenz $\omega$ gemessenen

\footnotetext{
${ }^{13}$ Ein Verfahren mit dieser Bezeichnung wird sonst zur Bestimmung des thermischen Transports allgemein in dielektrischen Materialien Cah90, speziell z. B. in dünnen Filmen CKA94, LC97, KFN99 oder auch Kohlenstoff-Nanoröhren Cho+06 verwendet, hat dort aber eine besondere Messgeometrie und unterscheidet sich daher von der hier beschriebenen Methode. Eine kurze Abgrenzung dazu befindet sich im Anhang in Kap. A.2

${ }^{14}$ Die folgenden Ausführungen nehmen zu einem großen Teil die genannte Veröffentlichung als Grundlage.

${ }^{15}$ Für die experimentelle Beschreibung einer solchen Messung siehe auch Kap. 3.1
} 


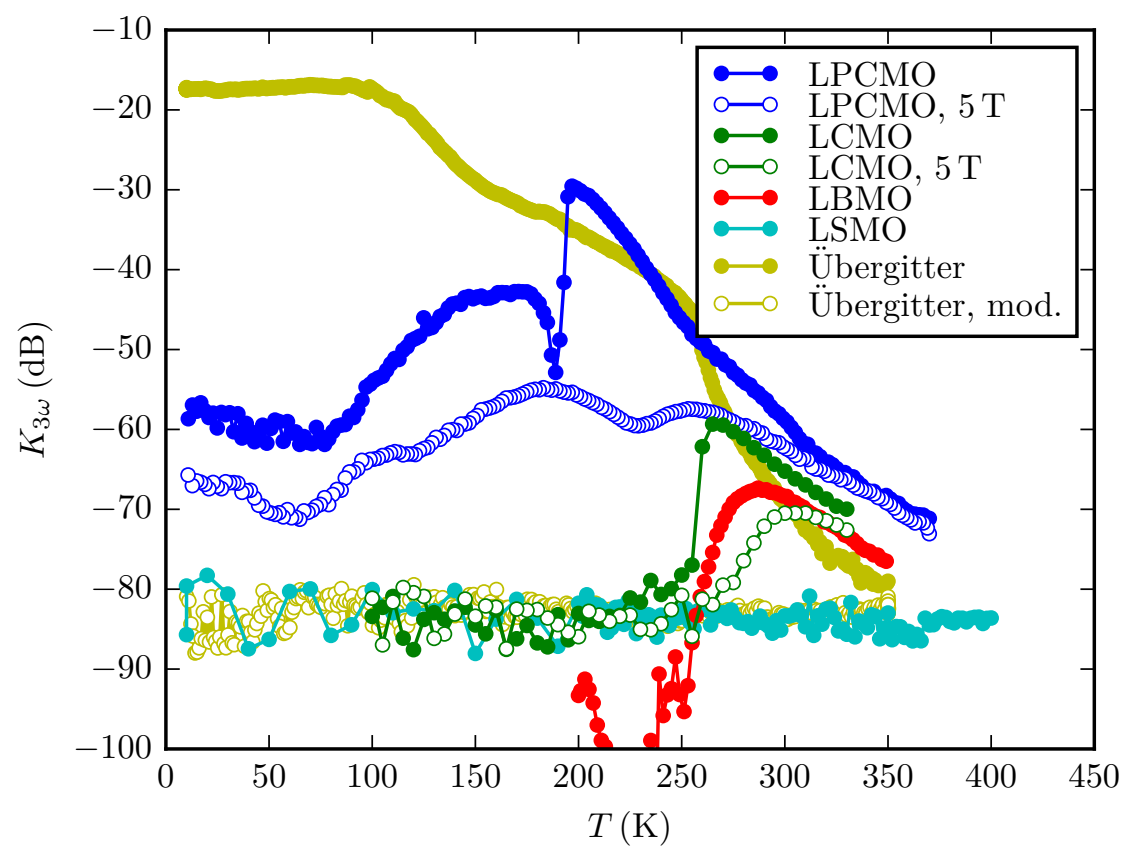

Abbildung 2.10.: Dritter harmonischer Koeffizient ${ }^{16} K_{3 \omega}=U_{3 \omega} / U_{\omega}$ für einige Manganate. Am Metall-Isolator-Übergang zeigt sich für LPCMO LCMO und LBMO ein stark erhöhter Wert, bei LSMO hingegen nicht. Ein Magnetfeld unterdrückt das Signal. Ebenso zeigt ein konventionelles Übergitter aus $\mathrm{LCMO}$ und $\mathrm{BaTiO}_{3}$ einen starken Anstieg, das modifizierte Übergitter mit zusätzlichen Zwischenlagen LMO jedoch nicht Geh+10. MoshnYAGA et al. Mos+09 argumentieren, dass der Koeffizient die Dichte der korrelierten Polaronen widerspiegelt. Nach [MS11].

Spannung $U_{\omega}$, welches man dritten harmonischen Koeffizienten

$$
K_{3 \omega}=\frac{U_{3 \omega}}{U_{\omega}}
$$

nennt. Die Daten dieser Messungen ${ }^{16}$ zeigt Abb. 2.10 und dort erkennt man das erhöhte Signal für die korrelierte Materialien ${ }^{17}$ LPCMO, LCMO und LBMO, aber nicht für LSMO, Ebenso kann ein Magnetfeld den Koeffizienten am Übergang unterdrücken, wie es vom CMR-Effekt bekannt ist, und tatsächlich ist seine Größe für den dritten harmonischen Widerstand $R_{3 \omega}=U_{3 \omega} / I$ vergleichbar oder sogar zwei Größenordnungen über dem von $R_{\omega}=U_{\omega} / I$.

Nun handelt es sich um eine Messung, bei welcher der Probe ein definierter alternierender Strom aufgeprägt wird, indem zum Beispiel ein im Vergleich zur Probe großer

\footnotetext{
${ }^{16}$ Bei dieser logarithmischen Auftragung ist $K_{3 \omega}=20 \log \left(U_{3 \omega} / U_{\omega}\right) \mathrm{dB}$.

${ }^{17}$ Siehe dazu auch Kap. 2.1.5 und Abb. 2.7.
} 
Vorwiderstand verwendet wird, sodass dieser den Strom zu

$$
I(t)=I_{0} \cos (\omega t)
$$

bestimmt. Dabei wird der Spannungsabfall $U=R I$ über der Probe gemessen. Ist jetzt aber der Widerstand nichtlinear, also abhängig vom Strom bzw. äußeren Feld und nehmen wir an, dass dieser Zusammenhang

$$
R(I)=R_{\Omega}+A I^{2}
$$

sei ${ }^{18}$ - wobei Terme höher Ordnung vernachlässigt werden -, so ergibt sich für die gemessene Spannung

$$
U=R(I) \cdot I=R_{\Omega} I+A I^{3}
$$

und damit

$$
U(t)=R_{\Omega} I_{0} \cos (\omega t)+A I_{0}^{3} \cos ^{3}(\omega t) .
$$

Mit dem trigonometrischen Additionstheorem erhält man daraus

$$
U(t)=\underbrace{\left(R_{\Omega} I_{0}+3 / 4 A I_{0}^{3}\right)}_{=U_{\omega}} \cos (\omega t)+\underbrace{1 / 4 A I_{0}^{3}}_{=U_{3 \omega}} \cos (3 \omega t),
$$

sodass mit einem Lock-in-Verstärker die erste harmonische Spannung, die aufgrund von $R_{\Omega} \gg A I_{0}^{2}$ im Wesentlichen den ohmschen Anteil $U_{\omega}=: R_{\omega} I_{0} \approx R_{\Omega} I_{0}$ liefert, und die dritte harmonische Spannung $U_{3 \omega}$ bestimmt werden kann. Und tatsächlich konnten Moshnyaga et al. Mos+09] messen, dass $U_{3 \omega} \propto I_{0}^{3}$, was die zuvor gemachte Annahme rechtfertigt.

Jetzt ist noch zu klären, was der Ursprung dieser Nichtlinearität sein könnte und dafür sei nochmal erwähnt, dass gerade beim Auftreten von korrelierten Polaronen $U_{3 \omega}$, genauer $K_{3 \omega}$, stark erhöht ist. Nun ist bekannt, dass der Jahn-Teller-Effekt durch Veränderung der Raumladungen am zentralen Manganion ein Quadrupolmoment hervorruft [KK82] und aus der nichtlinearen Optik weiß man, dass Quadrupolmomente quadratisch an ein externes Wechselfeld koppeln, was in Gl. 2.5) dem zweiten Term entspricht. Damit liegt die Schlussfolgerung nahe, dass $R_{3 \omega}:=U_{3 \omega} / I_{0} \propto N_{\mathrm{CP}}$, wobei $N_{\mathrm{CP}}$ die Zahl der Quadrupolmomente und somit die Zahl der korrelierten Polaronen ist. Außerdem lässt sich aus der berechtigten Annahme, dass durch korrelierte Polaronen die Mobilität der Ladungsträger stark eingeschränkt ist und daher die Leitfähigkeit proportional zu $N-N_{\mathrm{CP}}$ ist, wenn $N$ die Gesamtzahl der Ladungsträger ist, ein Ausdruck für den

\footnotetext{
${ }^{18}$ Es können in dieser Darstellung nur gerade Terme auftreten, da ungerade zu einem hier unphysikalischen negativen Widerstand führten. Anschaulich kann man sich vorstellen, dass sich z. B. bei einem Zusammenhang $I \propto U^{2}$ für den negativen Ast der Parabel ein negativer Widerstand ergibt, da dort die Spannung negativ, der Strom aber positiv wäre. Folglich wären in diesem Beispiel nur ungerade Exponenten physikalisch sinnvoll.
} 
Gesamtwiderstand gewinnen

$$
R \propto \frac{1}{N-N_{\mathrm{CP}}} .
$$

Dieser lässt sich mit $N_{\mathrm{CP}} \ll N$ entwickeln zu

$$
R \propto \underbrace{\frac{1}{N}}_{=R_{\omega}}+\underbrace{\frac{N_{\mathrm{CP}}}{N^{2}}}_{=R_{3 \omega}},
$$

wo man die Summanden aus Gl. (2.5) erkennen kann. Also erhält man

$$
K_{3 \omega}=\frac{R_{3 \omega}}{R_{\omega}}=\frac{N_{\mathrm{CP}}}{N}
$$

und folglich entspricht in diesem Modell der dritte harmonische Koeffizient $K_{3 \omega}$ der Dichte korrelierter Polaronen.

\subsection{Optische Anregungen}

Als letzter Punkt sollen optische Eigenschaften und mögliche optische Anregungen in den Manganaten diskutiert werden, wofür im Wesentlichen wellenlängenabhängige Absorptions- und Reflexionsmessungen an verschiedenen Manganatsystemen bei zum Teil variierender Temperatur betrachtet werden. So untersuchten MiLdner et al. [Mil+15] mithilfe optischer Absorptionsspektroskopie die optische Leitfähigkeit in PCMO für unterschiedliche Dotierungen, wovon für $x=0.35 \mathrm{Abb}$. 2.11 einen kleinen Auszug mit entsprechender Analyse zeigt. Dabei erklärten sie den Verlauf der Daten mit vier unterschiedlichen Anregungen, die sie bestimmten Übergängen in der berechneten Bandstruktur zuwiesen: An die beiden Anregungsbanden höherer Energie (C) und (D) im Ultravioletten wurde je ein Lorentz-Oszillator angepasst und ihnen die Übergänge $\mathrm{O}-2 p \rightarrow t_{2 g}^{\downarrow}$ bzw. O- $2 p \rightarrow e_{g}^{\downarrow}$ zugeordnet ${ }^{19}$. Jedoch liegen die typischen Energieskalen von Polaronen eher im Infraroten, weswegen die hohen Energien hier nicht besonders interessant sind. Außerdem könnten noch Absorptionsbänder bei höheren Energien auftreten, deren Flanken zum untersuchten Bereich beitragen, und auch aus diesem Grund seien Aussagen hierüber mit Vorsicht zu behandeln.

Demgegenüber gibt es aber auch noch zwei Peaks (A) und (B) im nahinfraroten Bereich bei zentralen Energien von $\hbar \omega_{\max }^{(A)} \sim 0.7 \mathrm{eV}$ und $\hbar \omega_{\max }^{(B)} \sim 1.5 \mathrm{eV}$, von denen der erste mit photonenunterstütztem Polaronen-Hüpfen identifiziert wird und der zweite einem Übergang zwischen den aufgespaltenen $e_{g}^{\uparrow}$-Niveaus ${ }^{20}$ entspricht ${ }^{21}$. Wie man erkennt, ist die zentrale Energie der Absorption (B) sehr passend zu Anregungen mit einer

\footnotetext{
${ }^{19}$ Eine ausführlichere Diskussion der optischen Eigenschaften und möglicher Übergange findet man in Qui+98.

${ }^{20}$ Streng genommen handelt es sich bei den Niveaus um hybridisierte Zustände zwischen O- $2 p$ und $\mathrm{Mn}-e_{g}^{\uparrow}$.

${ }^{21}$ Siehe auch Abb. 2.2 .
} 


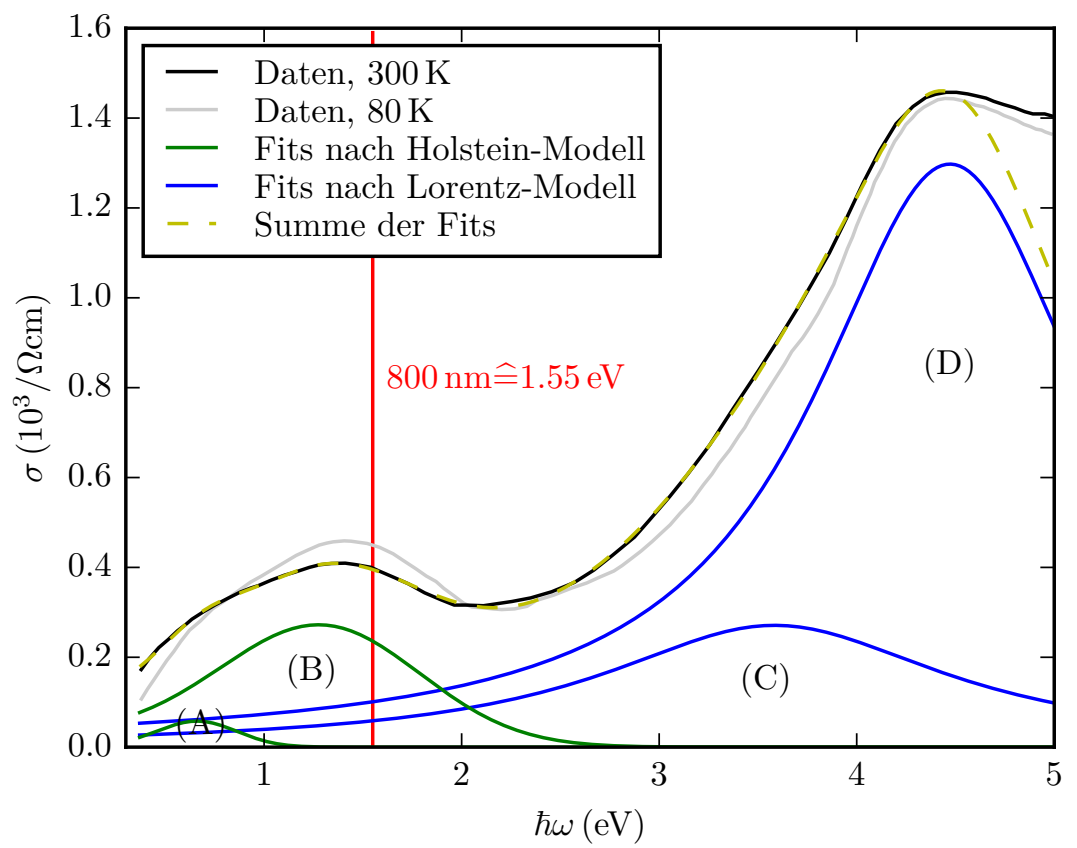

Abbildung 2.11.: Optische Leitfähigkeit eines PCMO-Films mit $x=0.35$. MiLdner et al. Mil+15 haben im Ultravioletten zwei Lorentz-Oszillatoren (blau) und im Nahinfraroten Anregungen von Kleinpolaronen (grün) angepasst. Die Summe dieses Modells beschreibt sehr zutreffend den Verlauf der Daten. Dieser ändert sich bei $80 \mathrm{~K}$ nur gering. Nach $[\mathrm{Mil}+15]$. 


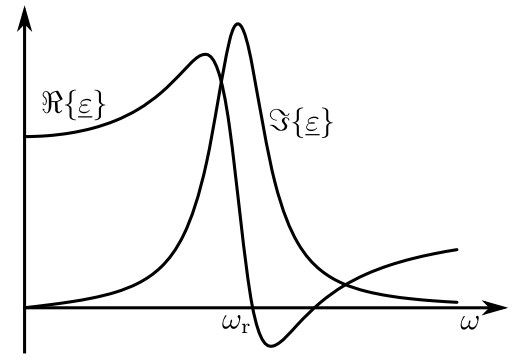

Abbildung 2.12.: Schematische Darstellung der komplexen dielektrischen Funktion in Abhängigkeit der Frequenz. $\Re\{\underline{\varepsilon}\}$ ist der Realteil, $\Im\{\underline{\varepsilon}\}$ der Imaginärteil und $\omega_{\mathrm{r}}$ die Plasmafrequenz.

Wellenlänge von $\sim 800 \mathrm{~nm}$ - was $1.55 \mathrm{eV}$ entspricht -, wie sie im Rahmen dieser Arbeit verwendet wurden (s. Kap. 3.5). Dafür sind natürlich auch die grundlegenden optischen Eigenschaften, vor allem aber die Absorption des untersuchten Materials entscheidend. Und um diese zu erhalten, sollte nun der komplexe Brechungsindex $\underline{n}=n+\mathrm{i} \kappa$ bekannt, sein, denn der Imaginärteil $\kappa$, der so genannte Extinktionskoeffizient, hängt direkt mit dem Absorptionskoeffizienten zusammen (s. Gl. (2.15)). Hierzu ist es allgemein üblich, die frequenzabhängige, komplexe Dielektrizitätsfunktion $\underline{\varepsilon}(\omega)$ zu ermitteln, aus der sich alle anderen optischen Eigenschaften ableiten lassen und die in Abb. 2.12 schematisch gezeigt ist. Möglich macht dieses das Programm RefFIT [Kuz15, in dem es $\underline{\varepsilon}(\omega)$ als Summe von Lorentz-Oszillatoren in der Form

$$
\underline{\varepsilon}(\omega)=\varepsilon_{\infty}+\sum_{j} \frac{\omega_{\mathrm{p}, j}^{2}}{\omega_{\mathrm{r}, j}^{2}-\omega^{2}-\mathrm{i} \gamma_{j} \omega}
$$

modelliert, wobei $\varepsilon_{\infty}$ die Hochfrequenz-Dielektrizitätskonstante, $\omega_{\mathrm{p}}$ die Plasmafrequenz, $\omega_{\mathrm{r}}$ die Resonanzfrequenz und $\gamma$ die Linienbreite ist. Bei der Modellierung wurden im Wesentlichen die Absorptionsbanden aus Abb. 2.11 als Lorentz-Oszillatoren angenommen und diese daraufhin durch die Fitroutine des Programms so angepasst, dass sich Verlauf der optischen Leitfähigkeit rechnerisch aus der komplexen Dielektrizitätsfunktion ergibt. Anschließend konnte über

$$
\begin{aligned}
& n(\omega)=\sqrt{\frac{|\underline{\varepsilon}(\omega)|+\Re\{\underline{\varepsilon}(\omega)\}}{2}} \\
& \kappa(\omega)=\sqrt{\frac{|\underline{\varepsilon}(\omega)|-\Re\{\underline{\varepsilon}(\omega)\}}{2}}
\end{aligned}
$$

der komplexe Brechungsindex ermittelt werden, was in Abb. 2.13 gezeigt ist. Die berechneten Werte sind plausibel und vergleichbar mit denen aus in-situ EllipsometrieMessungen von JungBAUER [Jun15] an LSMO, Außerdem kann nun leicht aus dem Extinktionskoeffizienten der Absorptionskoeffizient für $\lambda_{0}=800 \mathrm{~nm}$ berechnet werden

$$
\alpha=\frac{4 \pi \kappa}{\lambda_{0}}=7.56 \cdot 10^{6} 1 / \mathrm{m} .
$$

Ein Temperatureinfluss ist hier nicht besonders stark vorhanden, da sich die optische 


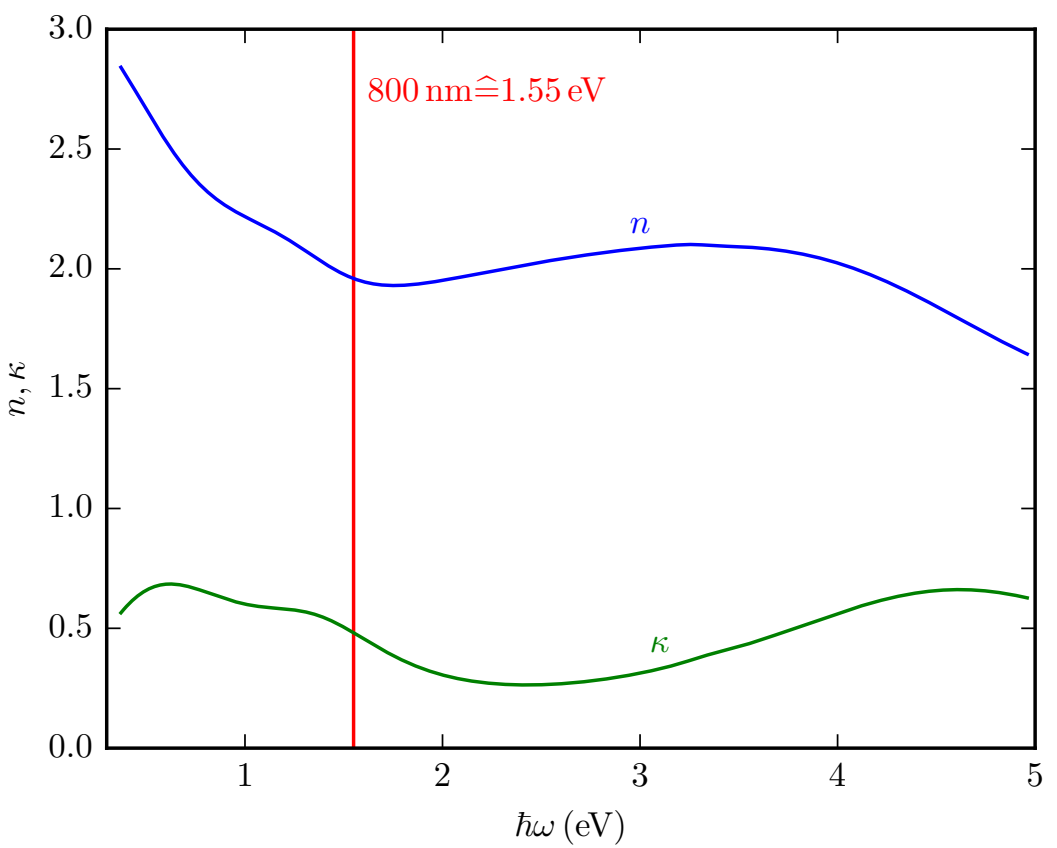

Abbildung 2.13.: Komplexer Brechungsindex $\underline{n}=n+\mathrm{i} \kappa$ eines PCMO-Films mit $x=0.35$ bei 300 K. Mittels RefFIT Kuz15] wurde eine dielektrische Funktion aus vier Lorentz-Oszillatoren an die Daten von Abb. 2.11 modelliert. Daraus lässt sich der komplexe Brechungsindex berechnen. Für $800 \mathrm{~nm}$ ist $n \approx 2$ und $\kappa \approx 0.5$. 
Leitfähigkeit bei $80 \mathrm{~K}$ kaum von der bei Raumtemperatur unterscheidet und lediglich im Nahinfraroten das spektrale Gewicht ein klein wenig zu höheren Energien verschoben ist, was durch die einsetzende Ladungs- und Orbitalordnung und das dadurch erschwerte Polaronen-Hüpfen begründet wird. Bei anderen Systemen wie LSMO oder LCMO hingegen wurden stärkere Veränderungen in der optischen Leitfähigkeit beobachtet Oki+95, Qui+98. Hier verschiebt sich das spektrale Gewicht mit sinkender Temperatur zu niedrigeren Energien, was bedeutet, dass sich bei $1.55 \mathrm{eV}$ von $290 \mathrm{~K}$ auf $180 \mathrm{~K}$ eine Abschwächung der optischen Leitfähigkeit um $\sim 9 \%$ ergibt. Natürlich spielen dabei der Metall-Isolator-Übergang und die einsetzende magnetische Ordnung - was beides bei PCMO nicht vorhanden ist - eine wesentliche Rolle, zeigen ihren starken Einfluss jedoch erst deutlich unterhalb der Übergangstemperatur.

Des Weiteren ist noch zu erwähnen, dass sich über die Linienbreiten der Absorptionsbanden unter Umständen Aussagen über Vibrationsmoden, also Phononenanregungen, treffen lassen, da sie für die Verbreiterung verantwortlich sein könnten. So konnten dann Mildner et al. Mil+15] auch aus der Absorptionsbreite von (B) die richtige Größenordnung für typische Anregungsenergien der so genannten Stretching- und Breathing-Moden von $\sim 70 \mathrm{meV}$ [Oki+99] ermitteln. Mit kohärenter Anregung dieser Moden bei entsprechend niedrigen Energien und der damit verbundenen starken Änderung der Mn-O-Bindungslängen war es RINI et al. Rin+07] sogar möglich, lokal den Widerstand des Materials transient um fünf Größenordnungen zu senken, was sie durch die optische Leitfähigkeit nachweisen konnten. Dabei war die Zeitskala der Relaxation zurück in den isolierenden Zustand mit $4 \mathrm{~ns}$ zu lang für rein elektronische Änderungen, was folglich die Kopplung des Elektronensystems an das Gitter unterstreicht.

Nun ist noch der Einfluss der Dotierung und der Verlauf bei anderen Systemen zu diskutieren. Da sich mit der Dotierung auch die Anzahl der freien Ladungsträger bzw. ihre Mobilität verändert, ist dieses auch in den Absorptionsbanden im Nahinfraroten zu beobachten, die sich mit steigendem Anteil an $\mathrm{Mn}^{4+}$ zu niedrigeren Energien verschieben $[$ Jun+98, Mil+15]. Jedoch sieht das Spektrum dort für gleiche Dotierung in unterschiedlichen Systemen sehr ähnlich aus [Oki+95, Jun+98, Mil+15] und folglich gibt es bei $\left(\mathrm{La}_{1-y} \mathrm{Pr}_{y}\right)_{1-x} \mathrm{Ca}_{x} \mathrm{MnO}_{3}$ mit festem $x$ und variierendem $y$ keine Veränderung [Jun+14.

\subsubsection{Auswirkungen auf die Gitterstruktur}

Wie man bereits in Kap. 2.1.4 über Polaronen gesehen hat, übt das Elektronensystem aufgrund der elektronischen Wechselwirkungen über die Elektron-Phonon-Kopplung direkten Einfluss auf die Struktur aus. Dieser kausale Zusammenhang ist bemerkenswert, denn er ist genau entgegengesetzt zu der sonst üblichen Annahme der Born-OppenheimerNäherung, bei welcher die Struktur der Rumpfionen im Gitter den Zustand des Elektronensystems bestimmt. Und tatsächlich konnten LEONOV et al. [Leo+10] in ihren Rechnungen zeigen, dass der Grundzustand in dem prototypischen Manganat $\mathrm{LaMnO}_{3}$ von den elektronischen Korrelationen abhängig ist. So berechneten sie die Gesamtenergie des Systems mithilfe der Näherung GGA (für engl. Generalized Gradient Approxima- 


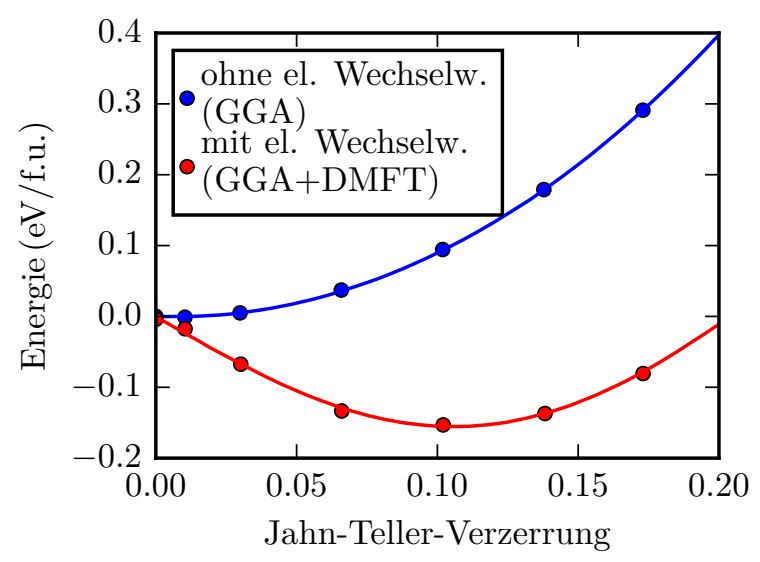

Abbildung 2.14.: Gesamtenergie von $\mathrm{LaMnO}_{3}$ in der paramagnetischen Hochtemperaturphase in Abhängigkeit der Jahn-Teller-Verzerrung. Zum einen berechnet ohne Berücksichtigung elektronischer Korrelationen (GGA für engl. Generalized Gradient Approximation, zum anderen mit Korrelationen (GGA+DMFT, DMFT für engl. Dynamic Mean Field Theory). Im ersten Fall ist der Grundzustand ohne Jahn-Teller-Verzerrung, im zweiten Fall mit. Nach Leo+10.

tion), welche elektronische Wechselwirkungen vernachlässigt, für verschieden ausgeprägte Jahn-Teller-Verzerrungen. Ebenso taten sie selbiges erneut mithilfe von $G G A+D M F T$ (DMFT für engl. Dynamic Mean Field Theory), was die Wechselwirkungen entsprechend berücksichtigt. Abbildung 2.14 zeigt ihre Ergebnisse. Dabei ergab sich für den Grundzustand nur im Fall der berücksichtigten elektronischen Wechselwirkung eine JahnTeller-Verzerrung von $\sim 10 \%$ mit einem Energiegewinn von $\sim 150 \mathrm{meV}$ pro Einheitszelle gegenüber dem unverzerrten System.

Nun liegt der Schluss nahe, dass man über die Beeinflussung dieser Korrelationen durch photoinduzierte Übergänge im Elektronensystem und die damit verbundenen Auswirkungen auf die Jahn-Teller-Polaronen mittelbar auch die Struktur des Systems verändern oder zumindest manipulieren kann. Dieses taten BEAUd et al. [Bea+14] bei ihren Pump-Abfrage-Experimenten an halbdotiertem PCMO mit nahinfraroter Pulsanregung und anschließender Darstellung der Struktur durch Kurzzeit-Röntgendiffraktometrie. Sie konnten zeigen, dass sich wenige Pikosekunden nach der Anregung die Kristallsymmetrie des Systems ändert, was sie über die Betrachtung eines zeitabhängigen Ordnungsparameters einem strukturellen Übergang zuschreiben konnten, der allerdings verschieden ist zu dem thermisch getriebenen Übergang.

Diese Erkenntnisse bilden die Grundlage für die Idee dieser Arbeit, mithilfe der nichtlinearen Transporteigenschaften, im Speziellen mithilfe des dritten harmonischen Koeffizienten, die Änderung in der Dichte korrelierter Jahn-Teller-Polaronen unter Laseranregung mit kurzen Pulsen nachzuweisen. 



\section{Experimentelle Methoden und Geräte}

Da es der Hauptaspekt dieser Arbeit war, die Einflüsse von Laserlicht auf Korrelationen in Manganaten mithilfe des elektrischen Transportes zu untersuchen, sollen im Folgenden die experimentellen Techniken und dafür verwendeten Geräte erläutert werden. Das umfasst unter anderem die Messelektronik und die verwendeten Laser. Des Weiteren wird ein selbst entwickelter Messeinsatz für einen kommerziellen Kryostaten vorgestellt. Ebenso wird die Probenherstellung und -präparation geschildert.

\subsection{Elektronischer Transport im Wechselfeld}

Um die korrelierten Polaronen, deren Natur in Kap. 2.1.4 beschrieben ist, zu untersuchen, wurde die über der Probe abfallende Spannung auf der dritten harmonischen Frequenz eines aufgeprägten Wechselstromes analysiert 11 Sinnvollerweise wird für eine solche Messung eine Geometrie aus vier Kontakten auf der Probe gewählt, weswegen das Verfahren Vierpunktmessung genannt wird.

Dabei wird an zwei außen liegenden Kontakten einer Probe ein Strom aufgeprägt und an zwei inneren Kontakten die abfallende Spannung gemessen, was mit Lock-inVerstärkern SR830 der Firma Stanford Research Systems realisiert wurde, wie Abb. 3.1 zeigt. Über den Ausgang des Verstärkers kann eine sinusodiale Spannung mit einer Amplitude zwischen 0 und $5 \mathrm{~V}$ ausgegeben werden, die über einen großen Vorwiderstand $R_{\text {vor }} \gg R_{\text {Probe }}$ an die Probe geführt wird. Dadurch soll ein definierter Strom durch die Probe fließen, der nicht von der Temperaturabhängigkeit des Probenwiderstandes beeinflusst wird, sondern nur durch den Vorwiderstand gegeben ist. Dieses Signal wird

\footnotetext{
${ }^{1}$ Den theoretischen Hintergrund dieser Messmethode erläutert Kap. 2.2
}

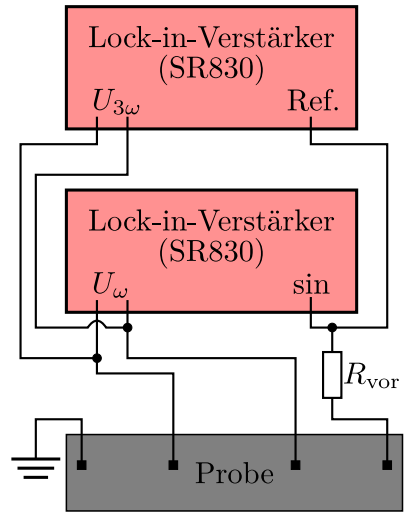

Abbildung 3.1.: Schema der Messgeometrie für elektrischen Transport im Wechselfeld. Das Sinussignal eines Lock-in-Verstärkers wird als Anregung auf die Probe geprägt, wobei der zweite äußere Kontakt durch den Lock-in-Verstärker auf Erdpotential liegt. An den inneren Kontakten wird mit einem der Verstärker die fundamentale, mit dem anderen die dritte harmonische Spannung gemessen. Über einen großen Vorwiderstand ist gewährleistet, dass der Strom auch bei Änderung des Probenwiderstandes konstant bleibt. 


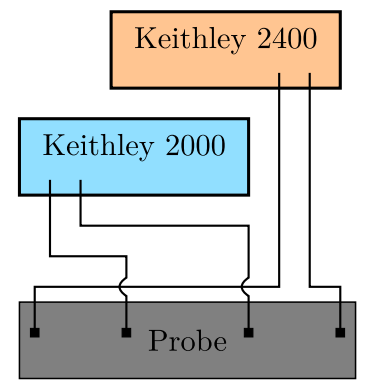

Abbildung 3.2.: Schema der elektrischen Messungen im Gleichfeld. Ein Keithley versorgt die beiden äußeren Kontakte der Probe mit einer konstanten Spannung und ermittelt den fließenden Strom, wobei gleichzeitig mit einem Keithley 2000 an den inneren Kontakten der Spannungsabfall gemessen werden kann.

zusätzlich als Referenz in einen zweiten Lock-in-Verstärker geführt, sodass gleichzeitig an den inneren Kontakten mit dem einen Verstärker die fundamentale Spannung $U_{\omega}$ und mit dem zweiten die dritte harmonische Spannung ${ }^{2} U_{3 \omega}$ gemessen werden können. Dabei wird der Lock-in-Verstärker im differentiellen Modus betrieben, sodass er intern die Spannungsdifferenz der beiden innen liegenden Kontakte bildet ${ }^{3}$

Für die elektrischen Verbindungen kamen handelsübliche BNC-Kabel zum Einsatz, bei denen in den meisten Fällen das Signal auf dem Innenleiter anlag und die Schirmung geerdet war.

\subsection{Strom-Spannungs-Kennlinien}

Ebenso wurden Messungen mit konstantem elektrischen Feld durchgeführt. Dafür kam an den äußeren Kontakten ein Keithley Sourcemeter 2400 zum Einsatz, das eine vorgegebene Gleichspannung zur Verfügung stellte und den dabei den fließenden Strom bestimmte, sodass an den inneren Kontakten der Probe mit einem Keithley Multimeter 2000 die dort abfallende Spannung gemessen werden konnte.

\subsection{Frequenzverhalten periodischer Anregung}

Um den Teil der Antwort der Probe auf Lichtanregung zu untersuchen, der sich auf eher langsamerer Zeitskala entwickelt, wurde die folgende Messkonfiguration verwendet, die schematisch in Abb. 3.3 gezeigt ist: Durch die äußeren Kontakte wurde der Probe mithilfe eines Keithley Sourcemeter 2400 eine konstanter Gleichstrom von $I_{\text {source }}=10 \mu \mathrm{A}$ aufgeprägt. Gleichzeitig regte der Laser den Steg auf der Probe mit einer Frequenz $f=60 \mathrm{~Hz} \ldots 3 \mathrm{kHz}$ an, die durch einen Chopper vorgegeben wurden, sodass ein Lock-inVerstärker auf eben jener Frequenz eine Spannung an den inneren Kontakten der Probe messen konnte, die aufgrund der periodischen Anregung entsteht. So kann man Zugriff auf die Zeitskala der Prozesse bei der Anregung bekommen, die langsamer ablaufen, wie z. B. Aufheizen oder Wärmediffusion.

\footnotetext{
${ }^{2}$ Dieser Messgröße geschuldet wird die Messmethode auch als $3 \omega$-Messung bezeichnet.

${ }^{3}$ Am Gerät wird dieser Modus mit $A-B$ bezeichnet.
} 


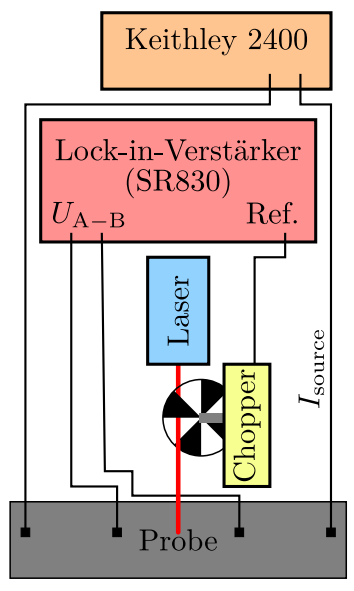

Abbildung 3.3.: Schema des Messaufbaus zur Bestimmung der Probenantwort auf periodische Laseranregung. Mit einem Sourcemeter wird der Probe ein konstanter Strom durch die äußeren Kontakte aufgeprägt. Dabei regt ein Laser den Steg der Probe an, wobei die Anregung periodisch durch einen Chopper mit änderbarer Frequenz erfolgt. Auf eben dieser Frequenz misst ein Lock-in-Verstärker die an den inneren beiden Kontakten aufgrund der Anregung auftretende Spannung.

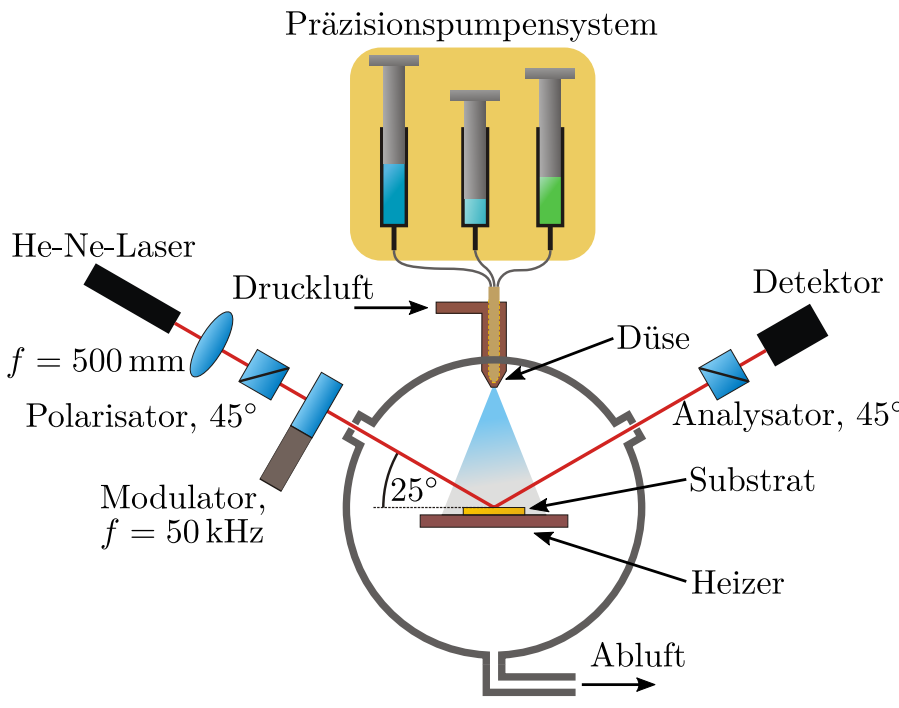

Abbildung 3.4.: Schema der MAD-Anlage zur Herstellung von dünnen Filmen. Über ein Präzisionspumpensystem können die in DMF gelösten Präkursoren, in denen die $\mathrm{zu}$ deponierenden Metalle gebunden sind, mikrolitergenau durch eine Düse auf das beheizte Substrat in der unter normaler Atmosphäre befindlichen Kammer gesprüht werden. Das Wachstum wird dabei über in-situ Ellipsometrie kontrolliert. Zeichnung erstellt von Camillo Ballani.

\subsection{Probenherstellung und -präparation}

Die Herstellung und Prozessierung der Proben wurden größten Teils von anderen Mitgliedern oder Gästen des Instituts durchgeführt. Dennoch soll im Folgenden zur besseren Einordnung ein kurzer Überblick über die dafür benutzten Techniken und Methoden gegeben werden.

\subsubsection{Metallorganische Aerosol-Deposition}

So wurden die beiden in dieser Arbeit untersuchten Dünnschichtproben von CAmillo Ballani bzw. Oleg Shapoval mithilfe der so genannten metallorganischen AerosolDeposition (MAD) (Schema in Abb. 3.4 hergestellt, die maßgeblich von MoshnYAGA et al. Mos+99 entwickelt wurde. Das besondere an dieser Technik ist, dass im Gegensatz zu den sonst gängigen Depositionsmethoden, wie der gepulsten Laserdeposition (engl. Pulsed 
Laser Deposition (PLD)), der Molekularstrahl-Epitaxie (engl. Molecular Beam Epitaxie $(M B E))$ oder dem Sputtern, in der Kammer der Anlage kein Vakuum, sondern normaler Druck und Luftzusammensetzung herrschen ${ }^{4}$. Daher weisen die hierdurch gewonnenen Proben, bei denen es sich ja um metallische Oxide handelt, keine Sauerstoffdefizite und eine hervorragende Qualität auf.

Für die Herstellung der Filme liegen nun die benötigten Metalle in organischen Verbindungen als so genannte Präkursoren vor, welche in entsprechender Zusammensetzung abgewogen und gemischt werden. Hierbei ist für das Verhältnis nicht nur die gewünschte spätere Stöchiometrie der Probe wichtig, sondern ebenso empirische Korrekturfaktoren, da die Präkursoren beim Bilden des Films auf der Substratoberfläche verschiedene Umsatzraten haben. Ist nun die richtige Mischung zusammengestellt, werden die Präkursoren in Dimethylformamid (DMF) aufgelöst, sodass diese Lösung durch Schläuche in das Innere der MAD-Kammer gepumpt werden kann, was mithilfe eines computergesteuerten Präzisionspumpensystems mikrolitergenau erfolgt. Dabei wird die Lösung mit Druckluft versetzt und durch eine Düse auf das auf $600 \ldots 100{ }^{\circ} \mathrm{C}$ aufgeheizte Substrat gesprüht, über dem sich dadurch ein Nebelkegel der Lösung bildet. Dort kommt es durch die hohe Temperatur zur Pyrolyse der organischen Präkursoren und die Metalle scheiden sich auf dem Substrat ab, welche dann durch Diffusionsprozesse auf der Oberfläche zusammen mit dem Sauerstoff aus der Umgebungsluft im Idealfall stufenweise den Film bilden. Währenddessen wird das Wachstum durch die Vermessung der Oberfläche mithilfe der Ellipsometrie überwacht. So kann man qualitativ hochwertige Oxidfilme mit Wachstumsgeschwindigkeiten von $1 \ldots 100 \mathrm{~nm} / \mathrm{min}$ [Mos+99] herstellen.

\subsubsection{Strukturierung}

Um nun den Steg und die Kontakte herzustellen, kam für die beiden in dieser Arbeit beschriebenen Proben die Elektronenstrahllithographie zum Einsatz. Jedoch wurden dafür zwei verschiedene Systeme und leicht unterschiedliche Verfahren eingesetzt.

\section{LBMO-Probe}

So verwendete Christin KALKenT für die Lithographie der LBMO-Probe, was im Rahmen ihrer Dissertation Kal13 geschah ${ }^{5}$, ein LEO Supra 35 der Firma Zeiss mit dem Lithographie-Modul Elphy-Plus der Firma Raith, sodass nach dem Auftragen eines Positivlacks mit Letzterem die Strukturen in den Lack geschrieben werden konnten. Anschließend wurde nach dem Entwickeln des Lacks die Struktur mittels Trockenätzen mit Argonplasma erstellt und danach der Lack mit Aceton im Ultraschallbad entfernt. Ebenso konnte mit einem Positivlack in einem weiteren Lithographieschritt eine Lackmaske erzeugt werden, mithilfe derer an die Position der Kontakte eine dünne Chromschicht als Haftvermittler und darauffolgend eine Goldschicht als Fläche für das Kontaktieren aufgedampft werden konnte. Auf diese Weise entstand ein dünner Steg

\footnotetext{
${ }^{4}$ Durch eine Erweiterung der Anlage können auch Proben unter geringerem Sauerstoffpartialdruck hergestellt werden, indem man die Kammer zu einem entsprechenden Teil mit Stickstoff flutet.

${ }^{5}$ Dort befindet sich auch im Kap. 4.4 eine detaillierte Beschreibung der Strukturierung.
} 
mit vier Kontakten (s. Abb. 4.4).

\section{LPCMO-Probe}

Bei der zweiten Probe wurden die zum Messen notwendigen Strukturen von Camillo BALlANI in einem Zwei-Schritt-Prozess nach folgendem Verfahren erzeugt, welches hier nur skizziert werden soll6; Nach dem Säubern der Oberfläche wurde die Schicht mit einem negativen Fotolack überzogen, auf den zur besseren Leitfähigkeit fürs Rasterelektronenmikroskop eine dünne Goldschicht aufgedampft wurde. So erfolgte dann auch das Schreiben der Strukturen durch Belichten des Lacks mit dem Elektronenstrahl an einer $e-L i N E$ von Raith, wonach anschließend die deckende Goldschicht wieder abgeätzt wurde. Danach wurde nach dem Entwickeln des Lacks die Struktur mit verdünnter Salzsäure freigestellt und dann der Lack mit Aceton wieder entfernt, welches den ersten Prozess abschloss.

Im zweiten Prozess, der zur Erstellung der Kontakte dient, wurde ein positiver Fotolack und ebenso die dünne Goldschicht aufgetragen. Anschließend konnte der Lack wieder im Rasterelektronenmikroskop belichtet werden, wofür zur Positionierung zuvor beim Strukturieren erzeugte Marker in den Ecken der Probe verwendet wurden. Daraufhin dampfte man nach Entfernen der oberen Goldschicht und der Entwicklung des Lacks zunächst wieder eine $5 \ldots 10 \mathrm{~nm}$ dünne Schicht Chrom als Haftvermittler und anschließend $30 \mathrm{~nm}$ Gold für die Kontakte auf. Zum Schluss beendete das abermalige Abheben des Lacks mit Aceton die Prozessierung und es lag eine strukturierte Probe mit großflächigen Goldpads für die Kontaktierung vor (s. Abb. 4.20).

\subsubsection{Kontaktierung}

Diese wurde mit einem Kulicke 85 Soffa 4523 durch Ultraschall-Wedge-Bonden hergestellt, bei dem ein dünner Metalldraht mit einer kleinen keilförmigen Spitze auf die Kontaktflächen gedrückt und unter Einwirkung eines kurzen Ultraschallpulses dort verschweißt wird. Dabei kam auf den Goldkontakten Aluminiumdraht mit einem Durchmesser von $33 \mu \mathrm{m}$ zum Einsatz.

\subsection{Laseranregung}

Zur Anregung der Proben wurden zwei Femtosekunden-Laser mit Titan-Saphir-Kristall im nah-infraroten Wellenlängenbereich, d. h. etwa $800 \mathrm{~nm}$, verwendet, von denen sich einer, ein Femtolasers Femtosource fusion 20, in Göttingen und der zweite, ein Coherent $\operatorname{Reg} A$ 9040, bei der Arbeitsgruppe Münzenberg in der Universität Greifswald befindet. Dabei unterscheiden sich beide Systeme deutlich in Leistung und Pulsenergie. Zunächst

\footnotetext{
${ }^{6}$ Für eine genauere Darstellung sei auf den Abschlussbericht des IFOX-Projektes BJM15 verwiesen, der die Prozessierung detailliert schildert, wobei jedoch bei der hier verwendeten Probe der initiale Schritt einer Pufferschicht ausgelassen wurde und anstelle von Argon-Ätzen chemisches Nassätzen zum Einsatz kam.
} 
soll jedoch knapp das generelle Prinzip der Erzeugung kurzer Pulse mit einem solchen Aufbau beschrieben werden.

\subsubsection{Funktionsweise eines Titan:Saphir-Lasers}

Ein Laser - für engl. light amplification by stimulated emission of radiation - besteht im Normalfall aus drei Komponenten: einer Energiepumpe, einem aktiven Medium und einem Resonator. Dabei führt die Energiepumpe dem Resonator, in dem sich das aktive Medium befindet, fortlaufend Energie zu, sodass die Lichtwelle in seinem Innern verstärkt werden kann. Hierfür muss das aktive Medium mindestens drei Energieniveaus besitzen, von denen man besetzte Zustände des Grundniveaus durch die Energiezufuhr in die Zustände des obersten Niveaus pumpen kann. Ist die Lebensdauer des obersten angeregten Zustandes kurz und die des mittleren lang, so kann es gelingen, mehr mittlere als Grundniveaus zu besetzen und es wird die so genannte Besetzungsinversion erreicht. Nun können durch stimulierte Emission diese Zwischenniveaus entvölkert und das dadurch entstehende kohärente Licht aus dem Resonator ausgekoppelt werden. Auf diese Weise erhält man kontinuierliches Laserlicht (engl. $c w=$ continuous wave).

Für die Erzeugung von kurzen Pulsen kann einfach die Energiepumpe gepulst oder die Verstärkungseigenschaft des Resonators mit einem so genannten Güteschalter (engl. q-switch) verändert werden, was zu Pulsdauern bis herunter in den Bereich von Nanosekunden führt. Will man jedoch zu kürzeren Zeitskalen gelangen, muss man ein breites Spektrum an Lasermoden kohärent überlagern, da ein großes Frequenzband zu einer kleinen Zeitdomäne und damit zu einem kurzen Puls führt. Folglich müssen im aktiven Medium mehrere Moden erzeugbar sein. Im Falle eines Titan:Saphir-Lasers, der verkürzt auch als Ti:Sa-Laser bezeichnet wird, ist das aktive Medium ein Saphir-Kristall, der mit Titanatomen dotiert ist. Dabei übernimmt der Kristall auch die Kopplung der Moden, also deren kohärente Überlagerung, da er wie eine Kerr-Linse wirkt und somit aufgrund nichtlinearer optischer Effekte intensives Licht und damit kurze Pulse stärker fokussiert, wohingegen der deutlich schwächere Dauerstrich-Anteil von einer Blende abgeschnitten wird [Dem07, S. 451].

\subsubsection{Femtolasers Femtosource fusion 20}

Bei dem Femtosource fusion von Femtolasers handelt es sich um einen kompakten Femtosekunden-Laser, der leicht auf einem kleinen Teil eines optischen Tisches Platz findet. Seine Pumpe ist ein Diodenlaser opus von Laser Quantum bei $532 \mathrm{~nm}$ mit einer maximalen Ausgangsleistung von $1.5 \mathrm{~W}$, die in die Kavität gestrahlt werden kann. So ergeben sich aus dem Resonator im Pulsmodus bei einer zentralen Wellenlänge von $\sim 795 \mathrm{~nm}$ und einer spektralen Breite von $\sim 9 \mathrm{~nm}$ eine Leistung von knapp $300 \mathrm{~mW}$ bei einer Pulsdauer von $\sim 120$ fs und einer Repetitionsrate von $\sim 74.9 \mathrm{MHz}$.

Die Besonderheit dieses Systems ist jedoch, dass das Laserlicht in eine spezielle Glasfaser eingekoppelt werden kann, sodass sich dennoch nach Durchlaufen der Faser eine kurzer Puls ergibt. Dafür kommt ein sogenannter photonischer Kristall mit der 


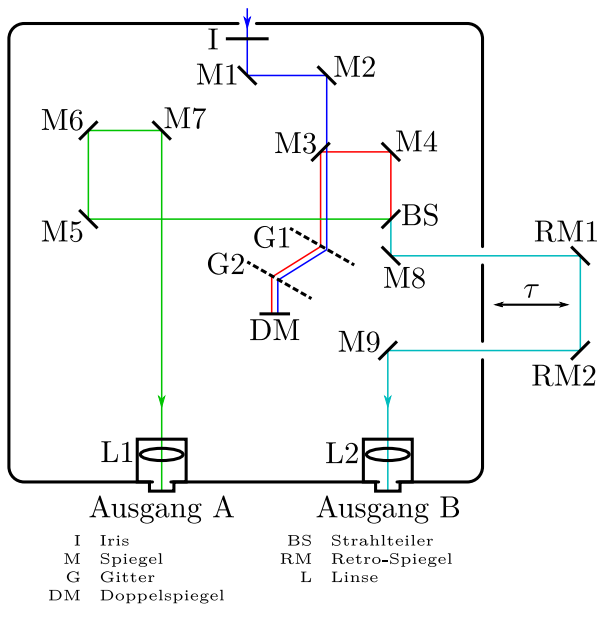

Abbildung 3.5.: Schema der Box zum Einkoppeln in den photonischen Kristall. Der Strahl tritt in der Zeichnung oben in die Box ein, wo er zunächst eine Iris (I) passiert und dann über zwei Spiegel (M1 \& M2) zu zwei Gittern (G1 \& G2) gelangt. Mithilfe dieser wird die in der Faser auftretende Dispersion kompensiert. Dabei werden die Gitter zweimal in unterschiedlicher Höhe (blauer und roter Strahl) durchquert, bevor ein Strahlteiler (BS) einen Teil (cyaner Strahl) auf eine Verzögerungsstrecke mit zwei Spiegeln (RM1 \& RM2) in den Ausgang B leitet, wohingegen der andere Teil (grüner Strahl) direkt über feste Spiegel (M5, M6, M7) in den Ausgang A läuft.

Bezeichnung LMA-PM-15 von NKT Photonics zum Einsatz, der als Lichtwellenleiter spezielle Eigenschaften aufweist. Denn dabei spielt nicht nur das gewählte Material eine Rolle, sondern auch die Überstruktur, die daraus hergestellt wird, was es zu einem so genannten Metamaterial macht. In diesem Fall ist eine Faser aus Quarzglas von nebeneinander liegenden Röhrchen durchzogen, sodass eine Art Hohlleiter entsteht. Dort kann sich über einen großen Querschnitt eine Einfach-Mode quasi an Luft polarisationserhaltend ausbreiten, sodass trotz der hohen Spitzenleistungen bei kurzen Pulsen die Nichtlinearitäten und damit verbundene starke Dispersion klein gehalten werden ${ }^{7}$ Jedoch muss auch hier die vorhandene Dispersion korrigiert werden, was durch zwei Gitter vor dem Einkoppeln passiert. So ist nach der Kavität auf dem optischen Tisch eine weitere Box platziert, in der die Einkopplung in die Faser realisiert wird. Wie Abb. 3.5 zeigt, teilt ein Strahlteiler nach der Dispersionskompensation durch die Gitter den Strahl auf, von denen einer über einen Linearversteller verzögert werden kann. Aber gerade diese Verzögerungsstrecke ist für das Einkoppeln in die Faser problematisch. Denn der Hersteller PI gibt für seinen Linearversteller M-531.DD einen Wert für das Neigen während des Verfahrens von 35 urad an, was bei einem Abstand zwischen RM2 und L2 von $\sim 20 \mathrm{~cm}$ zu einer Verschiebung des Strahls um $\sim 7 \mu \mathrm{m}$ auf L2 führt. Erschwerend kommt die Unebenheit des optischen Tisches hinzu, sodass sich das Neigen trotz sorgfältigster Justierung der Spiegel auf dem Verstelltisch (RM1 \& RM2) nur auf $\sim 140$ urad reduzieren lies. Dieser Umstand ist sehr kritisch, da die Glasfaser lediglich einen Kerndurchmesser von $15 \mu \mathrm{m}$ besitzt und mit einem Steckverbinder FC/APC 8 versehen ist, welcher äußerst empfindlich auf den Einkoppelwinkel ist. So konnte die Schwankung der Endleistung für verschiedene Positionen der Verzögerungsstrecke trotz umfänglicher Optimierung nicht unter $30 \%$ gebracht werden, weswegen der Ausgang $\mathrm{B}$ nicht für die Experimente genutzt wurde. Außerdem stehen generell baubedingt am

\footnotetext{
${ }^{7}$ Die sonst auftretenden Phänomene heißen Superkontinuumserzeugung (engl. supercontinuum generation) und Selbstphasenmodulation (engl. self-phase modulation).

${ }^{8}$ für engl. ferrule connector, angled physical contact
} 


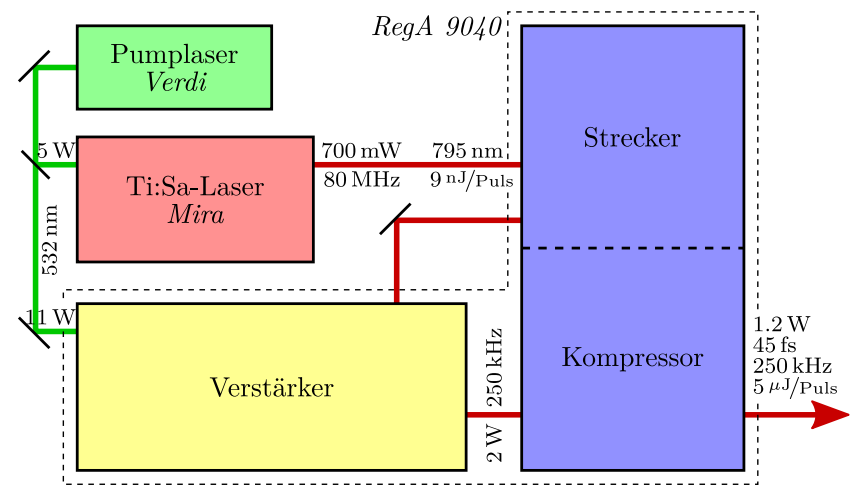

Abbildung 3.6.: Schema des Lasersystems Coherent RegA 9040. Der Pumplaser treibt eine Kavität mit Titan-Saphir-Kristall, dessen Pulse, nachdem sie in der Zeit gedehnt worden sind, abermals mithilfe des Pumplasers verstärkt werden. Anschließend werden die Pulse wieder gestaucht, sodass man $45 \mathrm{fs}$ bei $1.2 \mathrm{~W}$ und einer Repetitionsrate von $250 \mathrm{kHz}$ erhält.

Ende der Faser nur maximal 15 ..25 mW zur Verfügund9

\subsubsection{Coherent RegA 9040}

Dem gegenüber ist das Lasersystem RegA 9040 von Coherent in Greifswald für hohe Endleistungen konzipiert, da es einen aktiven Verstärker besitzt. Bei dem Aufbau, wie in Abb. 3.6 dargestellt, wird ein Titan-Saphir-Laser Mira von einem Festkörperlaser Verdi mit $532 \mathrm{~nm}$ und $5 \mathrm{~W}$ gepumpt, sodass dort Pulse bei $795 \mathrm{~nm}$ mit einer Repetitionsrate von $80 \mathrm{MHz}$ bei einer Leistung von ungefähr $700 \mathrm{~mW}$ entstehen, welche zunächst eine Pulsenergie von $9 \mathrm{~nJ}$ haben und als Ausgangspulse dienen. Nachdem diese durch den Strecker-Teil in der Zeit gedehnt worden sind, um die Spitzenleistung zur Schonung der optischen Bauteile zu senken, gelangen sie in den Verstärker. Dort werden die Pulse mithilfe weiterer $11 \mathrm{~W}$ aus dem Verdi-Pumplaser auf eine Leistung von $2 \mathrm{~W}$ bei einer Repetitionsrate von $250 \mathrm{kHz}$ verstärkt ${ }^{10}$, bevor sie im Kompressor-Teil wieder gestaucht werden. Am Ende erhält man Pulse bei $795 \mathrm{~nm}$ mit einer Länge von $45 \mathrm{fs}$ bei $1.2 \mathrm{~W}$ und $5 \mu \mathrm{J}$ pro Puls, welche als Freistrahl bis zum experimentellen Aufbau auf dem optischen Tisch geführt werden. Durch ein $\lambda / 2$-Plättchen und einen Polarisator kann dabei die Leistung auf einen gewünschten Wert abgeschwächt werden.

\subsection{Zeitliche Mittelung der Messgrößen}

Bei den Messungen der ersten und dritten harmonischen Spannung unter Laseranregung haben die verwendeten Gerätschaften - nämlich Lock-in-Verstärker und Laser - charakteristische Zeiten, die viele Größenordnungen auseinanderliegen. So sind bei einem Lock-in-Verstärker für Wechselfeld-Messungen mit moderaten Frequenzen unter $500 \mathrm{~Hz}$ Integrationszeiten von Millisekunden bis Sekunden zu wählen, über welche der Verstärker

\footnotetext{
${ }^{9}$ Dass die Leistung nach einiger Zeit etwas schwächer wurde, hat mit dem leichten Verschleiß des Steckerendes zu tun.

${ }^{10}$ Es handelt sich um einen regenerativen Verstärker, bei dem etwa jeder 300. Ausgangspuls in die Verstärkerkavität eingekoppelt und nach ungefähr 23 Umläufen die gesammelte Leistung ausgekoppelt wird. So kann man einen Verstärkungsfaktor von $\sim 10^{3}$ erreichen.
} 


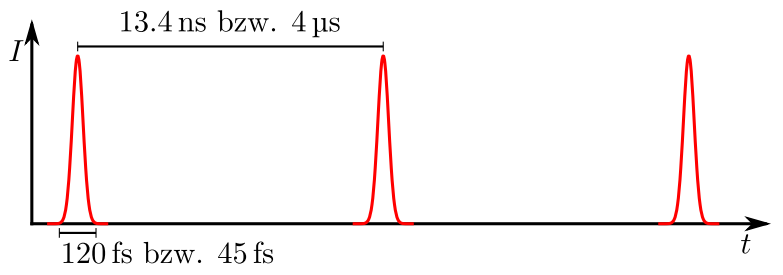

Abbildung 3.7.: Charakteristische Zeiten der Pulse bei den verwendeten Lasern. Sie ergeben sich aus Pulsdauer und Repetitionsrate.

die eingehenden elektrischen Signale zeitlich mittelt. Die verwendeten Laser hingegen arbeiten mit wesentlich kürzeren Perioden wie der Pulsdauer von $120 \mathrm{fs}$ bzw. $45 \mathrm{fs}$ und Repetitionsraten von $74.9 \mathrm{MHz}(\Delta t=13.4 \mathrm{~ns})$ bzw. $250 \mathrm{kHz}(\Delta t=4 \mu \mathrm{s})$. Entscheidend ist jedoch dabei, dass lediglich für die Dauer des Pulses eine Anregung stattfindet und für die verbleibende Zeit bis zum nächsten Puls die Probe quasi unbeleuchtet ist, was Abb. 3.7 veranschaulicht. Eine Anregung findet also bei dem Femtosource fusion 20 (Göttingen) nur für den Bruchteil von $9 \cdot 10^{-6}$ und bei dem RegA 9040 (Greifswald) sogar nur für einen Anteil von $1.1 \cdot 10^{-8}$ der Zeit statt, doch der Lock-in-Verstärker mittelt über die gesamte Zeit. Bei einer instantanen und maximalen Reaktion der Probe und ohne Relaxationszeit würde man demnach eine relative Änderung von ungefähr $10^{-3} \%$ bzw. $10^{-6} \%$ erwarten, was definitiv mit dem Lock-in-Verstärker nicht aufgelöst werden könnte. Aufgrund der langen Relaxationszeiten in den Manganaten, was in Kap. 5.4.1 diskutiert wird und von ultraschnellen Magnetisierungsmessungen bekannt ist Mül+09, ist es aber dennoch möglich, im Rahmen der Messgenauigkeit einen Effekt zu beobachten.

\subsection{PPMS-Messeinsatz}

Ein großer Teil dieser Arbeit war die Konstruktion eines Messeinsatzes für elektrische Messungen unter Laseranregung mit kurzen Pulsen in einem kommerziellen Kryostaten einem PPMS (für engl. Physical Properties Measurement System) von Quantum Design. Mit diesem können auf einfache Art und Weise vollautomatisch Temperaturen zwischen 2 und $400 \mathrm{~K}$ erreicht ${ }^{11}$ und magnetische Felder bis zu $9 \mathrm{~T}$ angelegt werden, was für die zu untersuchenden Manganate einen ausreichend großen Spielraum ließ. Dabei ist der Probenraum zylinderförmig mit einer Höhe von knapp $11 \mathrm{~cm}$ und einem Durchmesser von $2.54 \mathrm{~cm}$ (Abb. 3.8), wobei letzteres eine große Herausforderung darstellte. Denn auf so schmalem Raum, der sich weit im Innern des Dewargefäßes befindet, mussten Probenhalter, Optik und elektrische Verkabelung Platz finden. Zudem ist die Richtung des Feldes axial zur Probenkammer, was bedeutet, dass eine Dünnschichtprobe, für die das magnetische Feld in der Filmebene liegen soll, ebenso axial ausgerichtet sein und der Laserstrahl entsprechend um $90^{\circ}$ umgelenkt werden muss, um senkrecht auf die Probe zu treffen.

\footnotetext{
${ }^{11}$ Temperaturen über $300 \mathrm{~K}$ wären jedoch für den Messeinsatz problematisch, da die verwendeten Bauteile durch die Hitze Schaden nehmen könnten.
} 


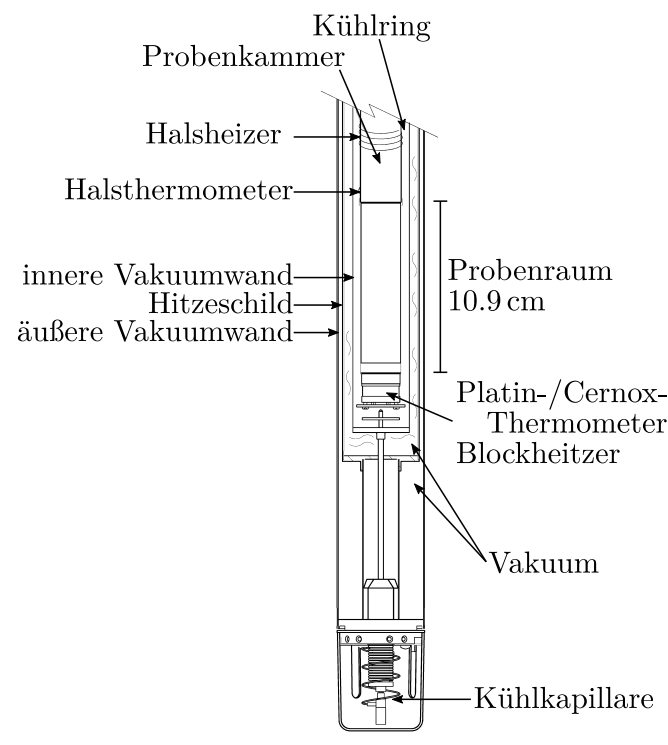

Abbildung 3.8.: Schema des PPMS-Probenkopfes, welcher in das Heliumbad gelassen ist, jedoch ohne Messeinsatz. Die Probenkammer kann bis auf $\sim 10$ mbar evakuiert werden und dann der Probenraum durch das Zusammenspiel aus zwei Heizern und einer Kühlkapillare, die ins Heliumbad reicht, vollautomatisch Temperaturen zwischen 2 und $400 \mathrm{~K}$ eingestellt werden. Dabei wird die Temperatur mit mehreren Thermometern überwacht. Nach Qua07.

\subsubsection{Messeinsatz}

In Anlehnung an einen originalen Messeinsatz für das PPMS wurde also ein eigener konstruiert, wie ihn Abbildungen 3.9 und 3.10 zeigen. Die kurzen Pulse aus dem Femtosource fusion (s. Kap. 3.5.2) werden über eine Glasfaser bis zum Kopf des Messeinsatzes geführt und dort mit einem Kollimator ausgekoppelt, sodass der verbleibende Pfad einen Freistrahl darstellt. So wird der Strahl über einen prismenförmigen Spiege 12 um $90^{\circ}$ umgelenkt und gelangt durch eine Linse 13 auf die Probe.

Letztere befindet sich auf einem L-förmigen Halter und kann durch diesen über drei Linearpositionierer ${ }^{14}$ in allen Raumrichtungen verschoben werden. Dabei bestehen diese Positionierer aus einem beweglichen Schlitten, der auf einer Schiene durch einen piezoelektrischen Kristall gegen den starren Grundkörper verschoben werden kann, wobei dieses nach dem Stick-Slip-Prinzip geschieht. Das heißt, bei einer langsam ansteigenden Spannung an dem piezoelektrischen Kristall verschiebt dieser den Schlitten, wohingegen bei der sprunghaften Depolarisation der Schlitten aufgrund der Trägheit in seiner Position verharrt. Die so verwendete Sägezahn-Spannung kann in Frequenz und Amplitude variiert und damit eine minimale Schrittweite von $50 \mathrm{~nm}$ bei Raumtemperatur erreicht werden kann. Allerdings gibt es keine Rückkopplung des Systems über die tatsächliche Verschiebung, weswegen alle Bewegungen nur indirekt über die Anzahl der instruierten Schritte bestimmt werden können. Jedoch unterscheiden sich deren Schrittweiten durch veränderte Temperatur oder Last und zusätzlich sind die Richtungen nicht reversibel,

\footnotetext{
${ }^{12}$ Thorlabs MRA05E03 mit einer Reflektivität von mehr als $99 \%$ bei $750 \ldots 1100 \mathrm{~nm}$

${ }^{13}$ Es wurden zwei Linsen getestet: zum einen eine Thorlabs A230B mit einer Fokuslänge von $f=4.51 \mathrm{~mm}$, zum anderen eine Thorlabs $A 375 B$ mit $f=7.5 \mathrm{~mm}$.

${ }^{14}$ Bei den Positionierern handelt es sich um piezoelektrische Aktuatoren der Firma attocube, die in sehr kompakter Bauform übereinander angeordnet sind. Dabei sind zwei Aktuatoren mit der Bezeichnung ANPx51 für eine horizontale Bewegung, ein dritter ANPz51 für eine vertikale.
} 

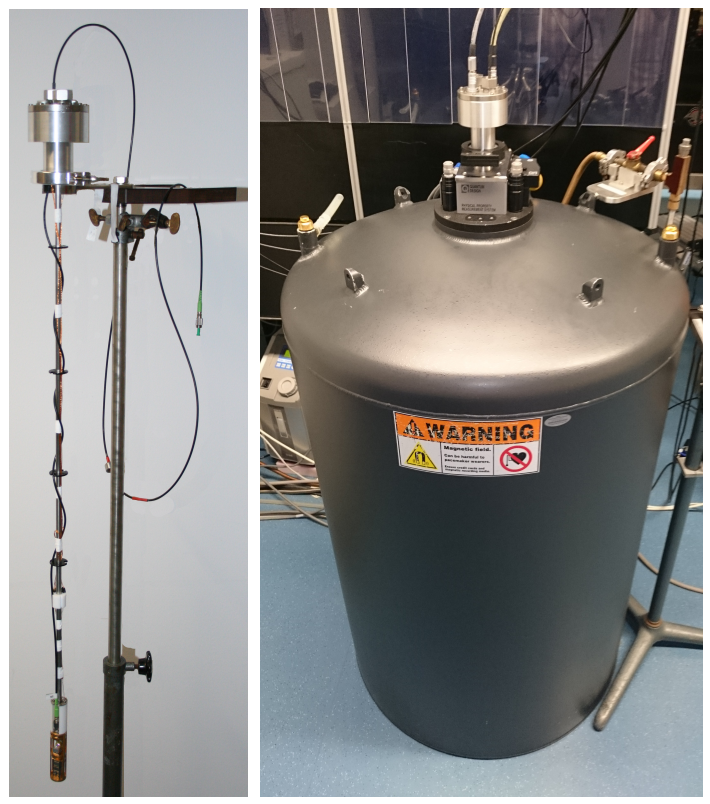

Abbildung 3.9.: Foto des Messeinsatzes außerhalb und im PPMS. Durch die oben befindlichen hermetischen Ports werden die Glasfaser und die elektrischen Messleitungen zum Messkopf geführt. Der stangenförmige Messeinsatz kann somit in die Probenkammer des PPMS eingebaut werden.
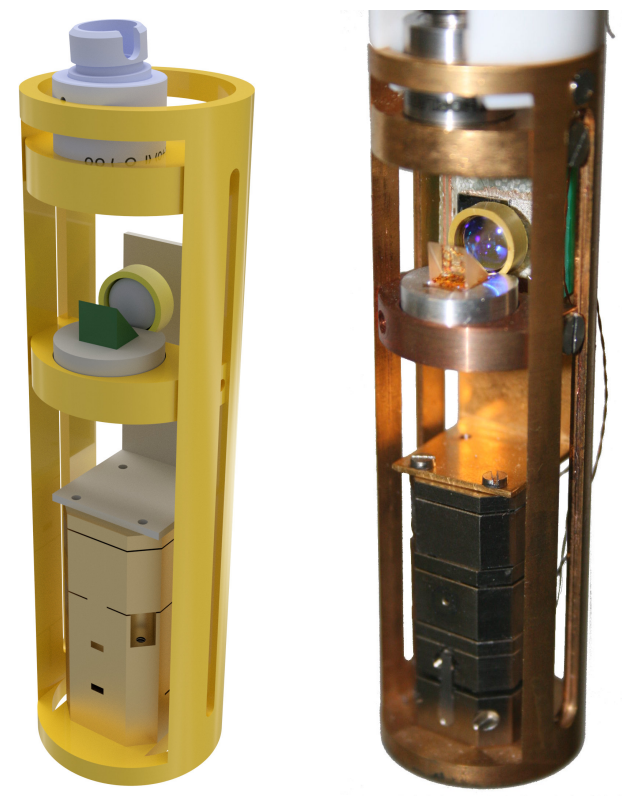

Abbildung 3.10.: CAD-Zeichnung und Foto des Messkopfes. In dem vergoldeten Kupferkäfig befinden sich zwei in der Höhe verschiebbare Plattformen, von denen die obere einen Kollimator trägt, an den die Glasfaser angeschlossen werden kann; die untere einen Drehteller mit $90^{\circ}$-Spiegel und Linse. Die auf den L-Halter gesteckte Probe kann mit den darunter liegenden piezoelektrischen Motoren in den drei Raumrichtungen verfahren werden. 
sodass ein positiver Schritt nie einem negativen entspricht. So ist es lediglich möglich, relative Positionierungen vorzunehmen, wobei nur vom Verschiebungsanschlag ausgehende Bewegungen reproduzierbar sind. Doch auch hierdurch erhält man die Position nur in der Einheit Schritte (engl. steps), die anschließend in Meter umgerechnet werden muss. Dafür wurde die Zahl der Schritte zwischen beiden Endanschlägen bestimmt, für welche der Verfahrweg aus dem Datenblatt der Piezoaktuatoren bekannt ist, woraus sich dann leicht der Umrechnungsfaktor bestimmen lässt 15

Der gesamte Messkopf ist in einen offenen Käfig aus vergoldetem Kupfer gefasst, der an der Seite zwei Langlöcher besitzt, in denen zwei Plattformen in der Höhe verschiebbar sind. Auf einer dieser Plattformen ist der Kollimator befestigt, auf der zweiten der drehbar gelagerte ${ }^{16}$ Prismenspiegel mit der Linse, sodass durch Verschieben dieser Plattform im Langloch die Höhe und durch Verdrehen des Tellers die horizontale Position grob eingestellt werden kann.

\section{Elektrische Verkabelung}

Da die Probenkammer des PPMS bis auf einen Druck von wenigen Millibar evakuiert wird, müssen die Mess- und Steuerleitungen hermetisch nach außen geführt werden, wofür entsprechend ausgestattete Buchsen der Firma Fischer Connectors verwendet werden. Um dabei die Steuersignale für die piezoelektrischen Positionierer von den Messleitungen zu trennen, wird je eine Buchse verwendet. Für die Leitungen im Innern kommt einfacher, lackierter Kupferdraht mit einem Durchmesser von $140 \mu \mathrm{m}$ zum Einsatz, bei dem je zwei Drähte für korrespondierende Kontakte, also z. B. I+ und I-, zu einem Leiterpaar verdrillt sind, um Störungen zu verringern. Zum Messkopf am unteren Ende des Einsatzes sind die Leitungen durch Microtech-Stecker und -Buchsen unterbrochen, damit man den unteren Teil z. B. für Wartung oder Reparatur vom Messeinsatz trennen kann. Die Messleitungen führen bis zu einem Sockel für die Probenplattform, mit dem sie fest verbunden sind. Dabei entsprechen sowohl Sockel und Plattform dem im PPMS verwendeten Standard ${ }^{17}$ für die Kontaktbelegungen, sodass kontaktierte Proben auch mit anderen Standardoptionen im PPMS vermessen und charakterisiert werden können.

\subsubsection{Vakuumdurchführung des photonischen Kristalls}

Eine Herausforderung war es, die Glasfaser ${ }^{18}$ vakuumdicht in den Probenraum zu führen, da über den Aufbau des kommerziell konfektionierten photonischen Kristalls keine Informationen verfügbar waren und aufgrund der filigranen Bauweise mit großer Vorsicht

\footnotetext{
${ }^{15}$ Die Zahl der Schritte wurde aus der Verfahrzeit, welche manuell gestoppt wurde, und der bekannten Schrittfrequenz ermittelt. Hierfür kann man aufgrund der menschlichen Reaktionszeit von $\sim 200 \mathrm{~ms}$ einen Fehler von $\sim 3 \%$ angeben.

${ }^{16}$ In der ersten Version befand sich anstelle des Drehtellers ein miniaturisierter Kipptisch, der jedoch aufgrund der geringen Distanz zur Probe den Strahl für eine Grobpositionierung nicht ausreichend verschieben konnte.

${ }^{17}$ Der Sockel ist ein Originalbauteil von Quantum Design für das PPMS, die Plattformen jedoch sind aus Leiterplatinen selbst gefertigte Replikate.

${ }^{18}$ photonischer Kristall mit der Bezeichnung LMA-PM-15 von NKT Photonics
} 


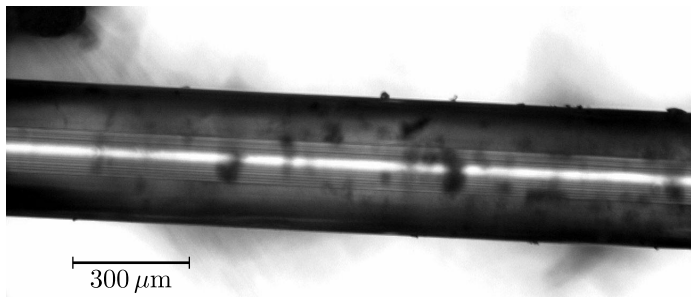

Abbildung 3.11.: Freigelegter photonischer Kristall $L M A-P M-15$. Von dem Gesamtdurchmesser von $350 \mu \mathrm{m}$ nimmt der innere Kern $15 \mu \mathrm{m}$ ein; der Mantel, den eine Röhrenstruktur durchzieht, hat eine Dicke von $107.5 \mu \mathrm{m}$ und der äußere Schutzüberzug $60 \mu \mathrm{m}$.

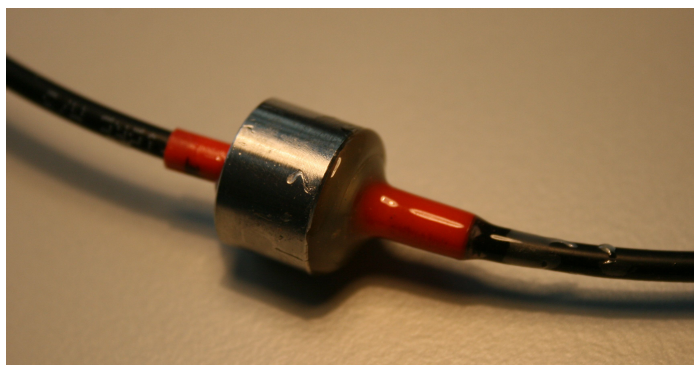

Abbildung 3.12.: Eingeklebte Glasfaser für die Vakuumdurchführung. Die freigelegte Faser ist in ein kleines rotes Röhrchen eingegossen, welches in einen Metallring geklebt ist.

vorgegangen werden musste. So ist unter der äußeren PVC-Hülle ein Fasergeflecht, welches zum Schutz gegen mechanischen Zug dient, vorhanden und darunter ein Plastikmantel, in dem der photonische Kristall lose verlegt ist. All diese Umhüllungen mussten entfernt werden, ohne den innen liegenden Kern (Abb. 3.11) zu beschädigen. Anschließend wurde dieser in einem kleinen Röhrchen mit Kleber vergossen und dieses wiederum in einen Metallring geklebt (Abb. 3.12), der über eine Quetschdichtung mit dem Messeinsatz hermetisch verschraubt werden kann.

\subsubsection{Kalibrierung des Thermometers}

Da die Probenplattform weiter von den systeminternen Thermometern des PPMS entfernt ist, befindet sich auf dem Sockel, der die Plattform aufnimmt eine weiteres benutzerinstalliertes Thermometer. Dabei handelt es sich um ein Widerstandsthermometer Cernox CX-1050 von Lake Shore in kompakter Bauweise ${ }^{19}$, welches für einen Temperaturbereich von $400 \mathrm{~K}$ bis $1.5 \mathrm{~K}$ und hohe magnetische Felder hervorragend geeignet ist.

Zuvor musste dieses jedoch kalibriert werden, wofür vor dem festen Einbau an den Messeinsatz sein Widerstand gegen das interne Thermometer des PPMS vermessen wurde, was in Abb. 3.13 gezeigt ist. Dabei ist zu erkennen, dass die Abkühl- und Aufwärmkurven direkt übereinanderliegen, was bedeutet, dass die Temperaturrampe langsam genug war, sodass sich das Thermometer immer auf der Temperatur des PPMS befand. Also wurde aus diesen Daten eine Widerstands-Temperatur-Kurve mithilfe eines Fits von Chebyshev-Polynomen abgeleitet und für die Temperaturbestimmungen benutzt. Weiter konnte diese Kurve ins PPMS übernommen werden, das darauf basierend die Temperaturregelung vornahm.

\footnotetext{
${ }^{19}$ In der so genannten $S D$-Form ist der dünne Film des Sensormaterials in einen hermetischen, metallischen Mantel in der Größe $3.2 \mathrm{~mm} \times 2 \mathrm{~mm} \times 1 \mathrm{~mm}$ unter Vakuum gefasst und bietet so gute thermische Ankopplung und mechanische Stabilität.
} 


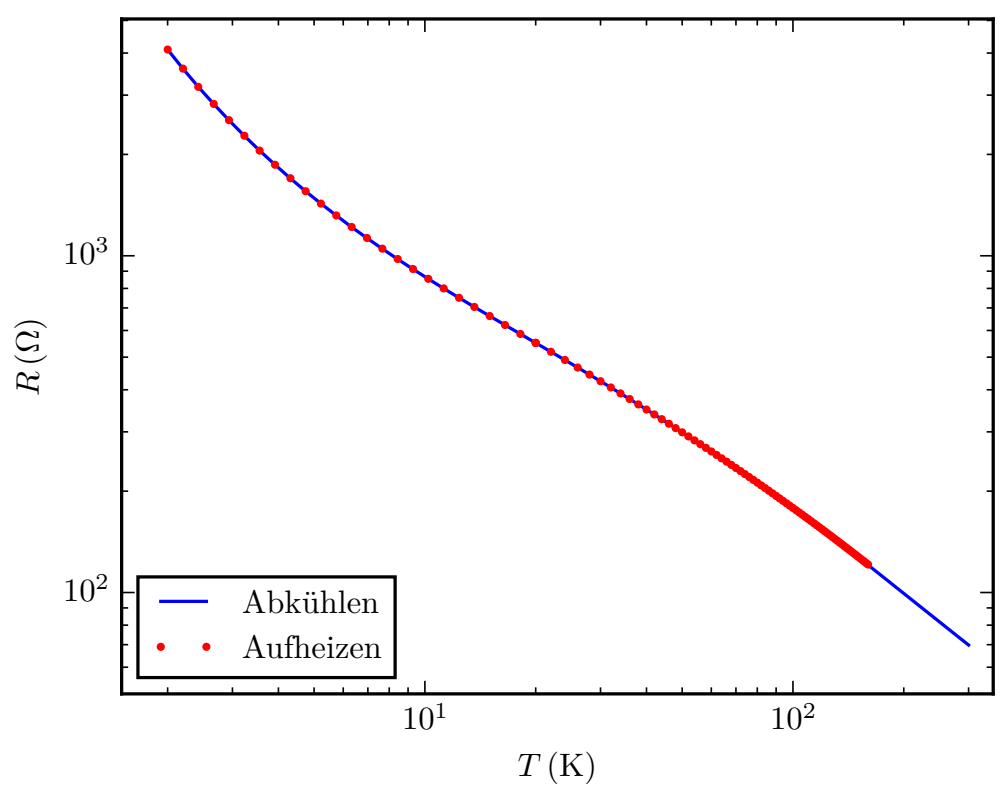

Abbildung 3.13.: Kalibrierung des Cernox-Thermometers. Der in Vierpunkt-Geometrie gemessene Widerstand des Thermometers ist gegen die Temperatur des PPMS aufgetragen. Die Kurven für das Abkühlen und Aufheizen liegen übereinander.

Außerdem wurde die Abhängigkeit des Widerstandes von größeren Magnetfeldern untersucht und in Abb. 3.14 die sich daraus ergebende scheinbare relative Temperaturänderung dargestellt. Jedoch ist dort zu sehen, dass diese Einflüsse vernachlässigbar klein sind. Und selbst bei Temperaturen bis hinunter zu $3.5 \mathrm{~K}$ bleibt die Änderung durch Magnetfelder bis zu $9 \mathrm{~T}$ kleiner als $0.5 \% 20$.

\subsection{Probenhalter mit Peltier-Kühlung}

Bei den Messungen mit dem Lasersystem in Greifswald ${ }^{21}$ konnte nicht auf das PPMS zurückgegriffen werden, zumal dort auch der Strahlenverlauf als Freistrahl realisiert ist, sodass ein anderer Weg der Temperaturkontrolle gewählt werden musste. Nun können mit stärkeren Peltierelementen Temperaturdifferenzen zwischen der heißen und der kalten Seite von bis zu $70 \mathrm{~K}$ erzeugt werden, was zumindest für Untersuchungen etwas unterhalb der Raumtemperatur ausreichend ist. Daher wurde ein Probenhalter mit einem geeigneten Peltierelement konstruiert, wie ihn Abb. 3.15 zeigt. Durch eine Wasserkühlung kann die von dem Element verursachte Wärme abgeführt und so die heiße Seite kalt gehalten werden. Die Probenaufnahme ist wieder an der imPPMS verwendeten angelehnt, sodass die Probenplattformen für beide Halter verwendet werden können.

\footnotetext{
${ }^{20}$ Untersuchung des Herstellers

${ }^{21}$ Für eine Beschreibung des Systems siehe Kap. 3.5.3.
} 


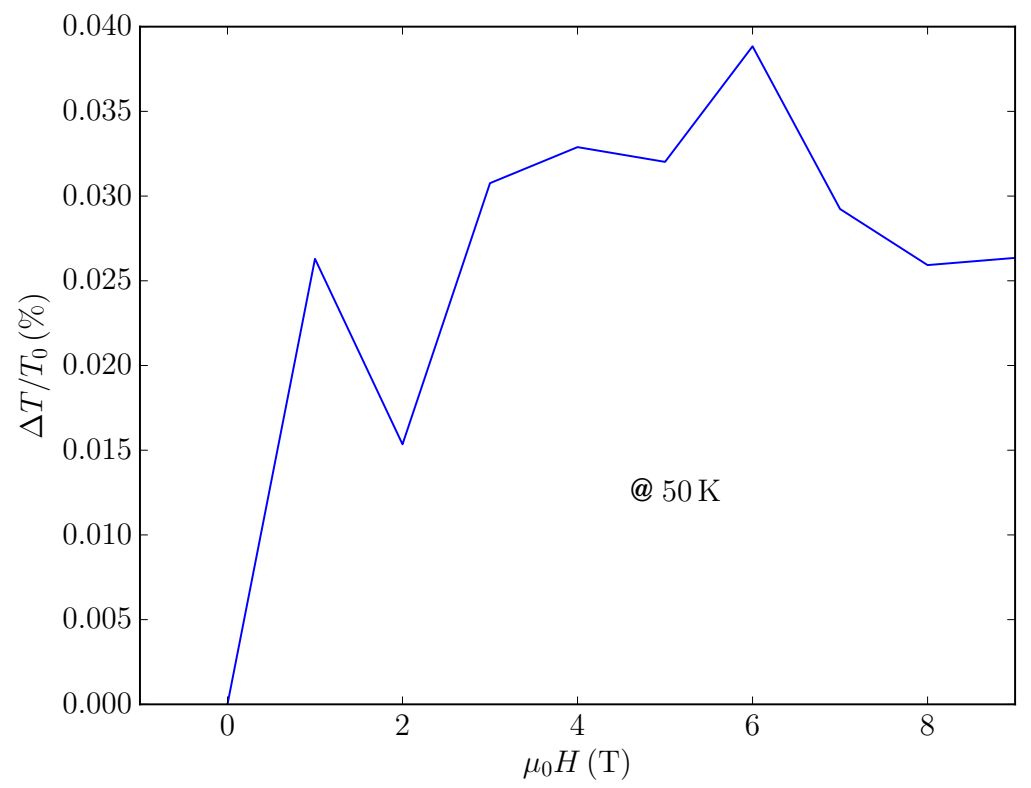

Abbildung 3.14.: Scheinbare relative Temperaturänderung des Cernox-Thermometers durch Magnetfeldeinfluss bei $50 \mathrm{~K}$. Wie erkennbar sind die Auswirkungen eines Magnetfeldes auf die Bestimmung der Temperatur aus dem Widerstand des Cernox-Thermometers vernachlässigbar klein.

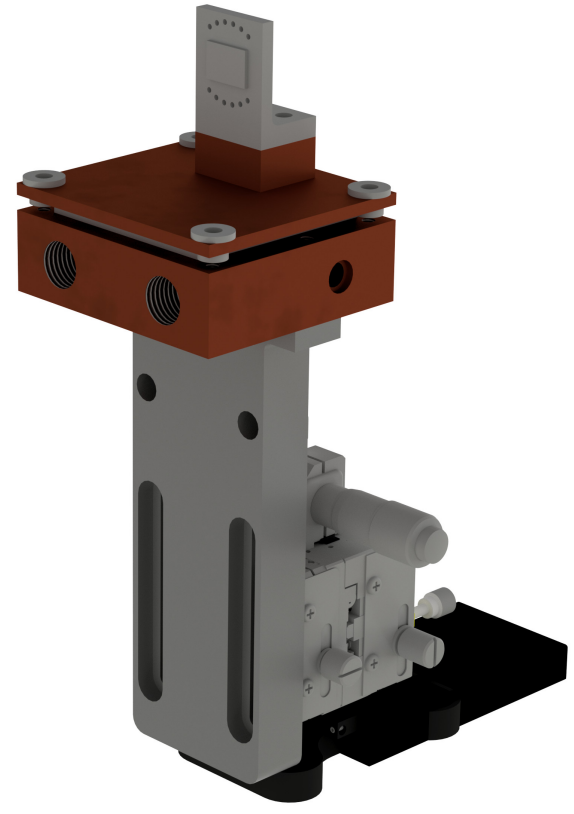

Abbildung 3.15.: CAD-Zeichnung des Probenhalters mit Peltier-Kühlung. Ein Peltierelement QC-127-2.015.0M von Quick-Ohm ist zwischen zwei Kupferplatten geklemmt, von denen die untere von Wasser durchflossen und somit gekühlt werden kann, um an der Probe Temperaturen bis hinunter zu $250 \mathrm{~K}$ zu erreichen. Auf der oberen Platte ist die Aufnahme für die Probenplattform aus Bornitrid gefertigt, was elektrisch isolierend, aber gut wärmeleitend ist. Dabei sind auch hier die Plattformen nach dem beim [PPMS verwendeten Design benutzbar. Durch die freistehende Aufnahme ist es außerdem möglich, den Halter zwischen die Polschuhe eines Elektromagneten zu bringen und so ein kleines Magnetfeld anzulegen. Weiterhin ist der gesamte Halter durch Translationstische in allen drei Raumrichtungen verschiebbar. Konstruktion und Zeichnung von CARSTEN MAHN und KAI DÖRNER. 
Lediglich auf ein dediziertes festes Thermometer wurde verzichtet und nur ein unter der Plattform festgeklemmtes Thermoelement verwendet, welches von einem Eurotherm 3508 ausgelesen und dazu benutzt wurde, um mithilfe einer PID-Regelung den Strom des Peltierelements so zu einzustellen, dass sich eine gewünschte Temperatur an der Probenplattform ergibt. Auch das Anlegen eines kleineren Magnetfeldes ist mit diesem Aufbau möglich, da die Aufnahme so kompakt gestaltet ist, dass sie leicht zwischen die Polschuhe eines vorhandenen Elektromagneten passt.

Ein Problem stellt jedoch bei einer Kühlung mit diesem Aufbau das Kondensieren und Gefrieren von Wasser aus der Umgebungsluft dar, wenn die Temperaturen sich dem Gefrierpunkt nähern. Daher wird der Probenraum von einer Stickstoffdusche mit seitlichen Lamellen eingefasst, sodass ein konstanter Gasstrom die Probe möglichst in eine Stickstoffatmosphäre hüllt.

\subsection{Fokusbestimmung}

Wenn ein Probensystem mithilfe eines Lasers angeregt werden soll, spielt immer auch die Fluenz, d. h. die Energiedichte auf Ort und/oder Zeit bezogen, eine wichtige Rolle. Eben dafür ist bei Puls-Lasern neben der Dauer der Pulse und der Repetitionsrate auch die laterale Ausdehnung des Anregungsflecks entscheidend.

Um diese zu bestimmen, wird oft die so genannte Rasiermessermethode benutzt, bei welcher der Laserfleck über eine scharfe Kante, die Bereiche unterschiedlich starker Reflektivität trennt, gezogen wird, während man die reflektierte Intensität aufzeichnet. Dabei erhält man für die Intensität als Funktion der Position des Laserflecks eine Sigmoidfunktion, die sich mathematisch aus der Faltung einer scharfen Stufenfunktion, welche die Kante repräsentiert, mit der Funktion des Strahlprofils, bei der es sich meistens um eine Gaußsche Funktion handelt, ergibt.

Nehmen wir also als Einhüllende für das elektrische Feld 22 der elektromagnetischen Welle des Laserlichts ein Gaußprofil

$$
E(x)=E_{0} \exp \left[-\frac{\left(x-x_{0}\right)^{2}}{w^{2}}\right]
$$

an mit der Maximalamplitude $E_{0}$, dem Schwerpunkt $x_{0}$ und der Strahltaille ${ }^{23} w$, woraus sich durch Quadrierung die Intensität

$$
I(x)=\frac{1}{2} c_{0} \varepsilon_{0} E(x)^{2}=I_{0} \exp \left[-\frac{2\left(x-x_{0}\right)^{2}}{w^{2}}\right]
$$

mit $I_{0}=1 / 2 \cdot c_{0} \varepsilon_{0} E_{0}^{2}$ ergibt. Diese wird nun mit der Stufenfunktion $\Theta$ gefaltet und man

\footnotetext{
${ }^{22}$ Das Profil wird nur entlang des radialen Verlaufs betrachtet und somit ist die axiale Abhängigkeit der Strahltaille hier nicht enthalten. Es handelt sich also um einen Schnitt durch den Strahl.

${ }^{23}$ Radius, bei dem die Intensität auf $1 / e^{2}$ abgefallen ist
} 
erhält24

$$
\begin{aligned}
\frac{\sqrt{2}}{\sqrt{\pi} w} \cdot(I * \Theta)(x) & =\frac{\sqrt{2}}{\sqrt{\pi} w} \cdot I_{0} \int_{\mathbb{R}} \mathrm{d} \tau \exp \left[-\frac{2\left(\tau-x_{0}\right)^{2}}{w^{2}}\right] \cdot \Theta(x-\tau) \\
& =\frac{\sqrt{2}}{\sqrt{\pi} w} \cdot I_{0} \int_{-\infty}^{x} \mathrm{~d} \tau \exp \left[-\frac{\left(\tau-x_{0}\right)^{2}}{w^{2} / 2}\right] \\
& =\frac{I_{0}}{\sqrt{\pi}} \int_{-\infty}^{\frac{x-x_{0}}{w / \sqrt{2}}} \mathrm{~d} t \exp \left[-t^{2}\right] \\
& =\frac{I_{0}}{\sqrt{\pi}}\left(\frac{\sqrt{\pi}}{2}+\int_{0}^{\frac{x-x_{0}}{w / \sqrt{2}}} \mathrm{~d} t \exp \left[-t^{2}\right]\right) \\
& =I_{0} \cdot \frac{1}{2}\left(1+\operatorname{erf}\left[\frac{x-x_{0}}{w / \sqrt{2}}\right]\right)
\end{aligned}
$$

mit der Gaußschen Fehlerfunktion

$$
\operatorname{erf}[z]:=\frac{2}{\sqrt{\pi}} \int_{0}^{z} \mathrm{~d} t \exp \left[-t^{2}\right] .
$$

Daraus kann man die volle Halbwertsbreit $2^{25}$ für das Strahlprofil zu

$$
\mathrm{FWHM}=\sqrt{2 \ln 2} w
$$

berechnen.

Nun kann diese Größe optimiert, d. h. verkleinert werden, indem man den Abstand der fokussierenden Linse und der Probe (s. Abb. 3.10) variiert, wobei der Strahldurchmesser am kleinsten ist, wenn sich die Probe genau im Fokus der Linse befindet. Zwar kann man so durch Verkleinern des Anregungsflecks die Energiedichte erhöhen, jedoch darf dieser nur so klein werden, dass die durch das Laserlicht anzuregende Struktur vollständig ausgeleuchtet wird.

\subsection{Abbilden der Probenoberfläche}

Da auf den Proben kleine Strukturen mit dem Laserlicht angeregt werden sollen, muss eine Positionierung des Anregungsflecks auf diesen erfolgen. Das kann jedoch nicht durch optische Kontrolle mit dem Auge oder mit einem Mikroskop geschehen, da zumindest für ersteres die Größe der Strukturen im Mikrometerbereich zu klein ist, aber auf jeden Fall das Streulicht des Lasers zu gefährlich für das Auge ist - gerade durch ein Mikroskop betrachtet. Also muss der Laserstrahl selbst als Sonde verwendet werden, um durch Rastern die Probenoberfläche abzubilden.

Ein Ansatz ist es, die elektrischen Kontakte der Struktur zu nutzen und an ihnen

\footnotetext{
${ }^{24}$ Der Vorfaktor $\sqrt{2} / \sqrt{\pi} w$ dient der Normierung.

${ }^{25}$ im Englischen FWHM für Full Width Half Maximum
} 


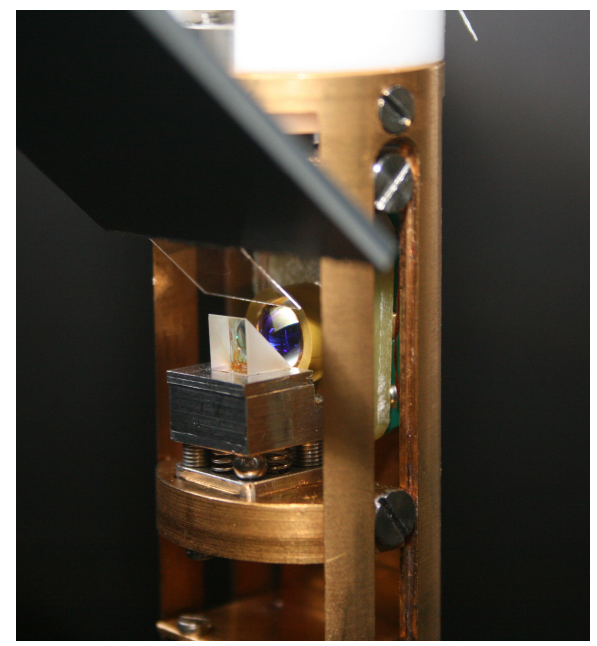

Abbildung 3.16.: Strahlteiler im Messkopf. Von außen in den Strahlengang eingeführt kann er das von der Probe zurück reflektierte Licht durch eine Linse auf eine Photodiode lenken und so das Abbilden der Probenoberfläche ermöglichen, wenn der Laserstrahl sie abtastet. In diesem Bild befindet sich auf der Zwischenplattform noch der Kipptisch anstelle des Drehtellers (siehe Fußnote 16 auf Seite 40 ).

direkt eine durch die Laseranregung induzierte Thermospannung zu messen, während die Probe mit dem Laserstrahl abgerastert wird. Da dabei jedoch nur kleine Spannungen zu erwarten sind, wird sich auch hier eines Lock-in-Verstärkers in Verbindung mit einer durch einen Chopper erzeugten optischen Modulation bedient. Allerdings ist diese Art der Abbildung nur indirekt und stellt besondere Anforderung an die physikalischen Eigenschaften der Probe in Bezug auf thermische Leitfähigkeit, spezifische Wärme und Seebeck-Koeffizient. Der große Vorteil dieser Methode ist jedoch, dass die Darstellung der Probe in-situ geschehen kann und somit auch bei wechselnden Temperaturen und Magnetfeldern im PPMS,

Eine weitere Möglichkeit besteht darin, das zurückreflektierte Licht der Probe einzufangen, auf eine Photodiode zu lenken und ebenfalls mit dem Laserstrahl über die Probe zu scannen. So erhält man ein Abbild bezüglich der Reflektivität, sodass z. B. Goldkontakte deutlich sichtbar werden und auch ein Unterschied zwischen Film und Substrat erkennbar ist. Natürlich kommt auch hier die Modulation durch einen Chopper und ein Lock-in-Verstärker zum Einsatz. Doch hierbei ist die Schwierigkeit, das reflektierte Licht einzusammeln, da die engen Platzverhältnisse zusätzliche optische Komponenten nahezu unmöglich machen. Folglich bleibt nur, mit einem von außen in den Strahlengang eingeführten Strahlteiler in Form eines kleinen Deckglases (s. Abb. 3.16), die Reflexion abzuzweigen und außerhalb mit einer Sammellinse und einer Photodiode einzufangen. Auf diese Art erhält man ein detailliertes Bild der Probenoberfläche und kann auch mit der in Kap. 3.9 beschriebenen Methode den Strahldurchmesser auf der Probe bestimmen. 


\section{Experimentelle Ergebnisse}

In diesem Kapitel sollen die durchgeführten Messungen mit ihren experimentellen Daten vorgestellt werden. Dabei wird auch auf offensichtliche Auffälligkeiten eingegangen und diese werden eingeordnet, jedoch erfolgen tiefer gehende Erklärungen und zusammenfassende Interpretation erst im darauffolgenden Kap. 5.

\subsection{Lanthan-Barium-Manganat}

Bei der ersten in dieser Arbeit untersuchten Probe handelt es sich um Lanthan-BariumManganat (LBMO) mit der Strukturformel $\mathrm{La}_{1-x} \mathrm{Ba}_{x} \mathrm{MnO}_{3}$ und der Dotierung von $x \approx 0.25$ auf einem Strontiumtitanat-Substrat (STO) mit (100)-Orientierung, welches mit der MAD-Technik von CAMillo Ballani hergestellt wurde円 Diese Dotierung weist, wie Abb. 4.1 zeigt, eine hohe Übergangstemperatur nahe Raumtemperatur zwischen einer paramagnetisch isolierenden Hochtemperaturphase und einer ferromagnetisch metallischen Tieftemperaturphase auf. Daher eignete sich die Probe für vorausgegangene Experimente mit begrenzter Kühlmöglichkeit, wie sie von KALKERT Kal13 durchgeführt wurden, welche hier überprüft und erweitert werden konnten.

\subsubsection{Grundlegende Charakterisierung}

Nun sollen zunächst einige grundlegende charakterisierende Eigenschaften der Probe betrachtet werden, um die Beschaffenheit beurteilen zu können.

\section{Oberfläche und Struktur}

So zeigen die Messungen mittels Röntgendiffraktometrie (Abb. 4.2), dass die Gitterkonstante $c \approx 3.913 \AA$ und die Dicke der Schicht zu $30 \mathrm{~nm}$ abgeschätzt werden kann.

Ebenso ist die Schicht sehr glatt und stufenweise gewachsen, wie man an der Aufnahme durch das Rastertunnelmikroskop in Abb. 4.3 sehen kann. Für die Gitterstruktur zeigen Raman-Messungen von Sebastian Merten, dass die Probe bei Raumtemperatur bis hinunter zu $77 \mathrm{~K}$ orthorhombisch ist.

Anschließend wurde von Christin Kalkert durch die Prozessierung der Probe, d. h. Strukturierung, ein kleiner Bereich mittels optischer Lithographie freigestellt und dort ein Steg mit einer Breite von $30 \mu \mathrm{m}$ und einer Länge von $120 \mu \mathrm{m}$ erzeugt, der an seinen Enden zwei größere Flächen aus dem Manganat aufweist (Abb. 4.4). Auf jede

\footnotetext{
${ }^{1}$ Die Nummer der Probe lautet $X 105$.
} 

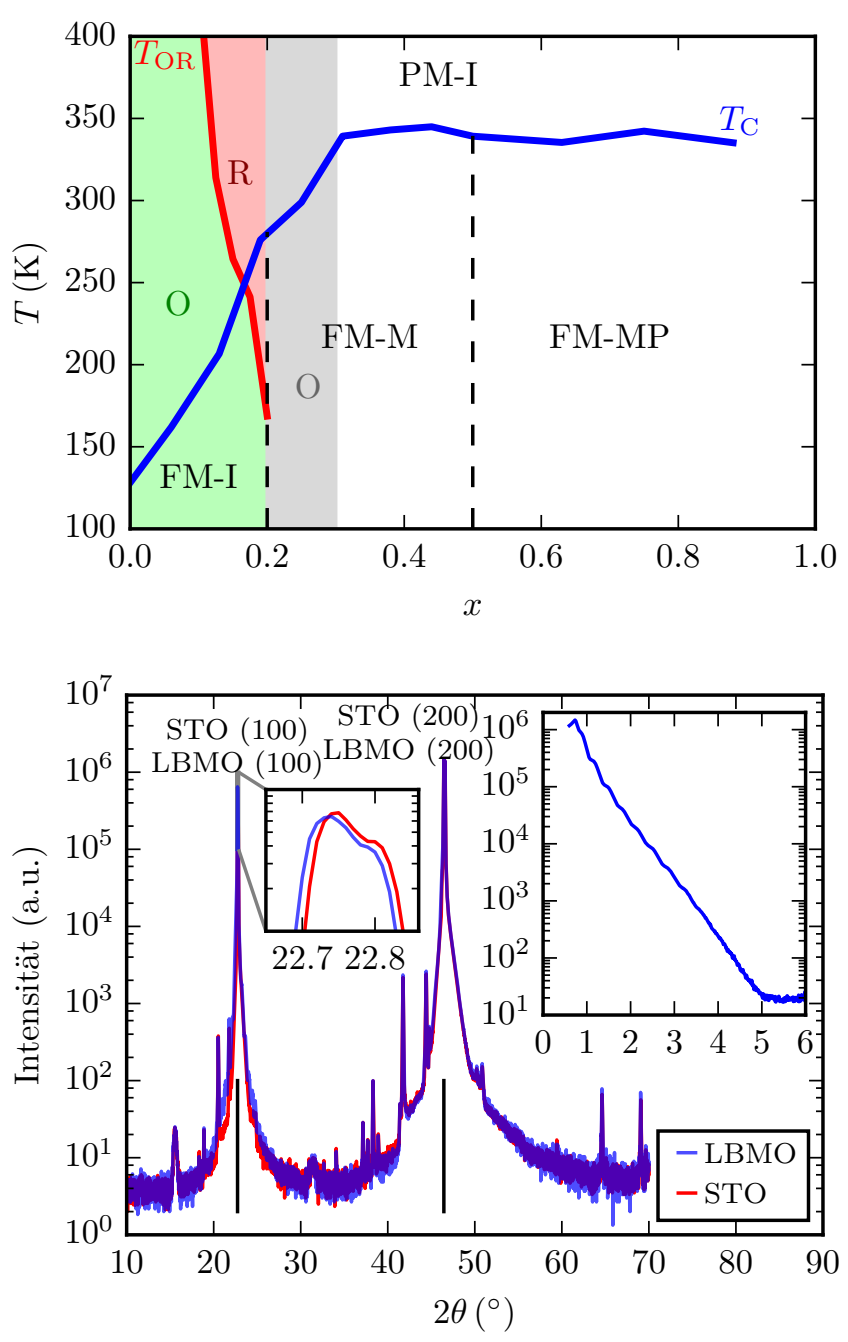

Abbildung 4.1.: Phasendiagramm von $\mathrm{La}_{1-x} \mathrm{Ba}_{x} \mathrm{MnO}_{3}$. Oberhalb der Phasengrenze ist das Material paramagnetisch isolierend (PI), bei tieferen Temperaturen wird es abhängig von der Dotierung $x$ ferromagnetisch isolierend (FI), ferromagnetisch metallisch (FM) oder ferromagnetisch mit Multiphase (FMP). Die Gitterstrukturen können orthorhombisch (O) oder rhomboedrisch (R) sein. Im grau markierten Bereich befindet sich die hier verwendete Probe. Für eine ausführlichere Diskussion siehe Kap. 2.1.5. Nach Ju+00.

Abbildung 4.2.: Röntgendiffraktometrie von LBMO mit Weitwinkelund Kleinwinkelmessung (Nebenbild). Aus Ersterer lässt sich die Gitterkonstante $c \approx 3.913 \AA$, aus Letzterer die Schichtdicke $30 \mathrm{~nm}$ bestimmen. Die Peaks von LBMO und dem Substrat STO fallen aufgrund der gemeinsamen Perowskit-Struktur nahezu zusammen, lassen sich im Detail jedoch trennen. Daten von Camillo Ballani.

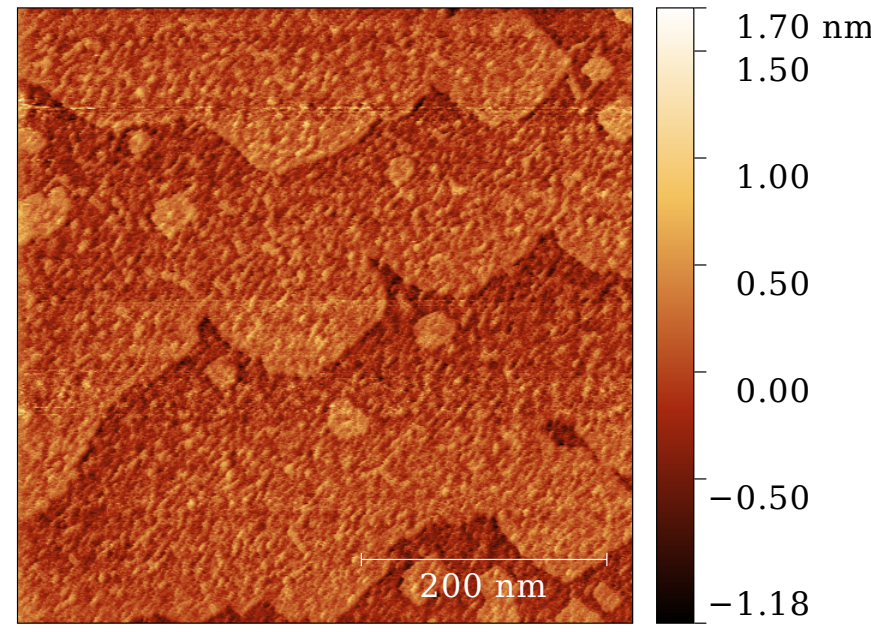

Abbildung 4.3.: Rastertunnelmikroskop-Aufnahme der LBMO, Probe. Es liegt Stufenwachstum vor und die Probe ist mit einer Rauigkeit von RMS $<2.7 \AA$ sehr glatt. Daten von Camillo Ballani. 


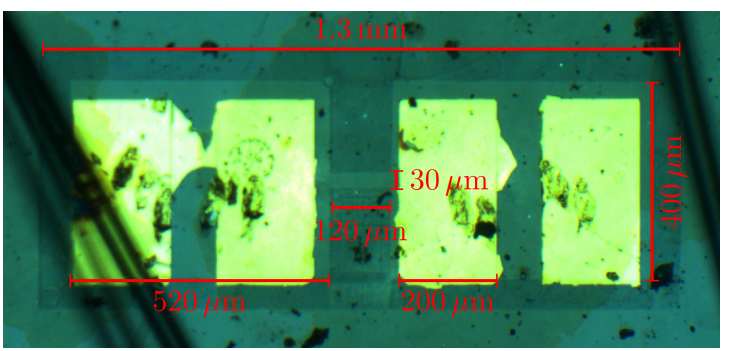

Abbildung 4.4.: Foto der Struktur auf der LBMO Probe. Im dunkler erscheinenden Bereich ist die Schicht weggeätzt und das Substrat ist direkt sichtbar, wobei der äußere Bereich wieder mit LBMO bedeckt ist. Die großflächigen Kontakte bestehen aus einer Schicht Chrom und Gold. Die Schatten auf dem Bild zeigen Bonddrähte, die zu einer anderen Struktur führen, wohingegen diese Struktur noch nicht kontaktiert ist.

dieser beiden Flächen sind jeweils getrennte Chrom-Gold-Kontakte aufgedampft, die mit Bonddrähten kontaktiert werden können, um eine elektrische Vierpunktmessung durchzuführen.

\section{Widerstand}

Das Temperaturverhalten des Widerstandes bietet eine grundlegende Charakterisierung, was in Abb. 4.5 gezeigt ist. Dabei wurde der Gleichstromwiderstand vor und nach der Strukturierung im PPMS mit der Widerstandsoption bei einem Anregungsstrom von $50 \mu \mathrm{A}$ beim Abkühlen gemessen. Hier zeigen die Messungen konsistent einen MetallIsolator-Übergang bei $T_{\mathrm{MI}} \sim 280 \mathrm{~K}$, welches den Maxima der Kurven entspricht. Und wie erwartet lässt sich der Übergang aufgrund des CMR-Effektes durch ein Magnetfeld unterdrücken und zu höheren Temperaturen verschieben, was hier einem $\operatorname{CMR}(5 \mathrm{~T}) \approx 57 \%$ entspricht. Weiterhin kann man aus dem Maximum des Widerstandstemperaturkoeffizienten (TCR für engl. Temperature Coefficient of Resistivity) $\mathrm{TCR}=1 / R \cdot \mathrm{d} R / \mathrm{d} T$ die Temperatur $T_{\mathrm{TCR}} \sim 258 \mathrm{~K}$ ermitteln, welche den Punkt des steilsten Anstiegs in der Widerstandskurve angibt.

\section{Magnetismus}

Bezüglich der magnetischen Eigenschaften ist ja bereits diskutiert, dass LBMO bei tiefen Temperaturen ferromagnetisch ordnet, und so lässt sich aus der Temperaturabhängigkeit der Magnetisierung die Curie-Temperatur und das Sättigungsmoment bestimmen. Dazu ist die entsprechende Kurve in Abb. 4.6 gezeigt und man erhält einen Wert von $T_{\mathrm{C}} \sim$ $279 \mathrm{~K}$, ebenso $m_{\mathrm{s}} \approx 2.23 \mu_{\mathrm{B}} / \mathrm{Mn}$. Für Letzteres müsste man jedoch, wie in Fußnote 10 von Kap. 2.1.5 erwähnt, bei einer Dotierung von $x \approx 0.25$ ein Sättigungsmoment von $3.75 \mu_{\mathrm{B}} / \mathrm{Mn}$ erwarten, von dem hier nur $60 \%$ erreicht werden. Allerdings wurde die Probe zum einen an einigen Stellen bereits lokal destruktiv untersucht? zum anderen ist ein Teil des Manganats aufgrund der Strukturierung durch das Ätzen entfernt worden. Weiterhin sind für die Bestimmung des Sättigungsmomentes die geometrischen Parameter wie

\footnotetext{
${ }^{2}$ Mithilfe von Laserablation und anschließender Massenspektrometrie mit induktiv gekoppeltem Plasma (engl. Inductively Coupled Plasma Mass Spectrometry, ICP-MS) hat CAMILlo BaLlani versucht, die genaue Komposition des Manganats zu bestimmen, was jedoch nicht erfolgreich war.
} 


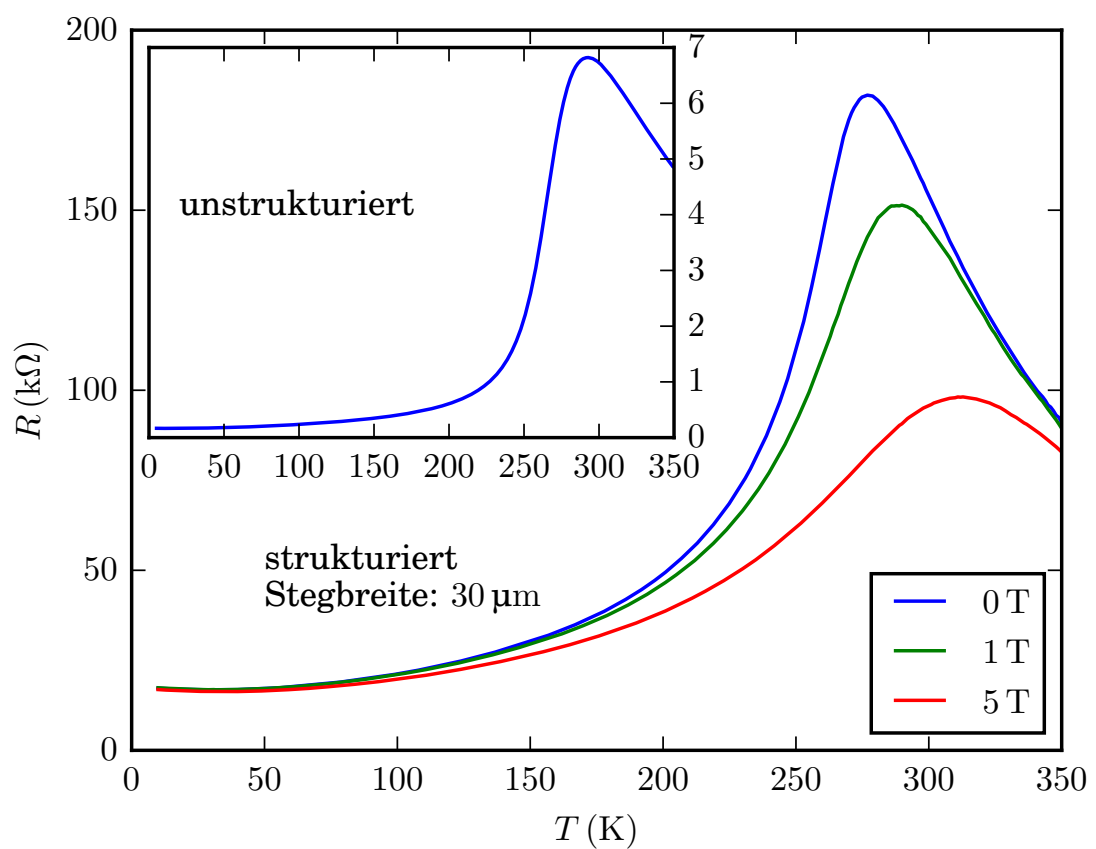

Abbildung 4.5.: Temperaturabhängigkeit des Widerstandes der LBMO-Probe. Leicht unterhalb von Raumtemperatur bei $T_{\mathrm{MI}} \sim 280 \mathrm{~K}$ zeigt die Probe den Metall-Isolator-Übergang, der in seiner Position auch durch die Strukturierung nicht beeinflusst wird. Der CMR-Effekt für $5 \mathrm{~T}$ ist $57 \%$. Der steilste Anstieg befindet sich bei $T_{\mathrm{TCR}} \sim 258 \mathrm{~K}$. 


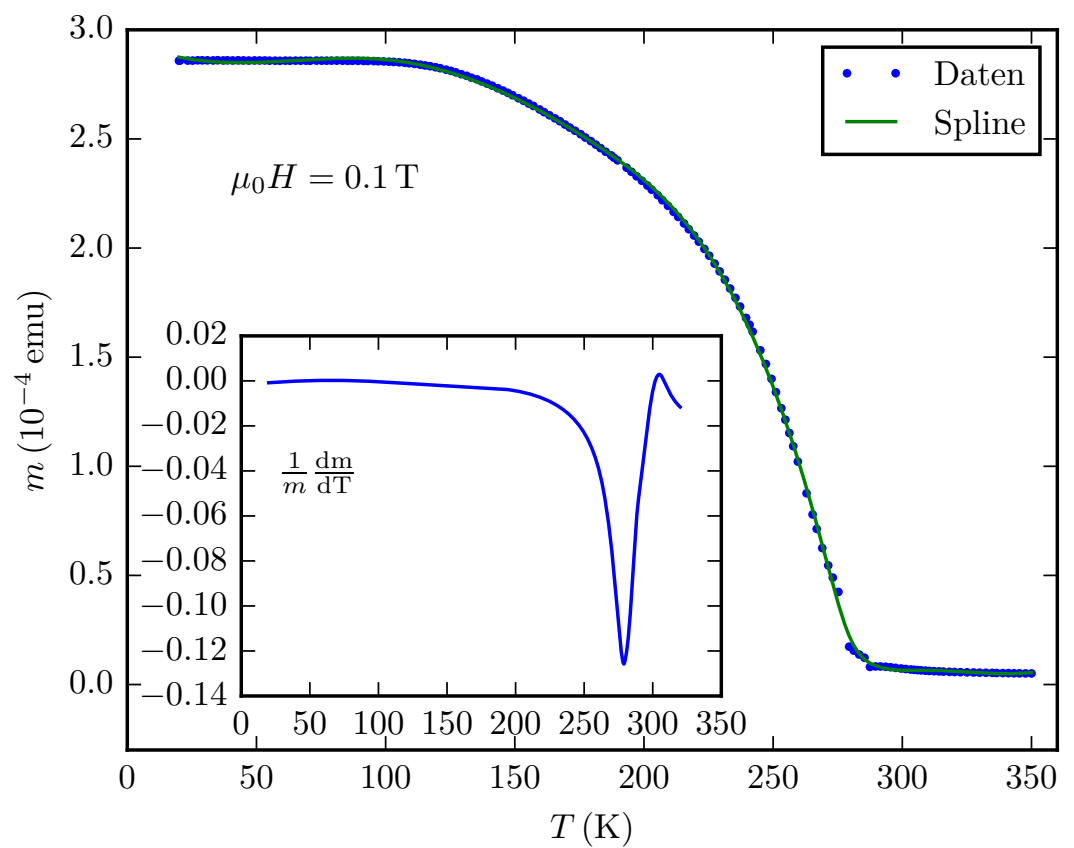

Abbildung 4.6.: Magnetisierung in Abhängigkeit der Temperatur von LBMO bei einem externen magnetischen Feld von $\mu_{0} H=0.1 \mathrm{~T}$. Streng genommen handelt es sich um das magnetische Moment der gesamten Probe. Nach Subtraktion des Substratbeitrages kann mithilfe eines Splines die relative Ableitung (Nebenbild) ermittelt werden, woraus sich eine Curie-Temperatur von $T_{\mathrm{C}} \sim 279 \mathrm{~K}$ bestimmen lässt. Als Sättigungsmoment wurde $2.23 \mu_{\mathrm{B}} / \mathrm{Mn}$ ermittelt. 

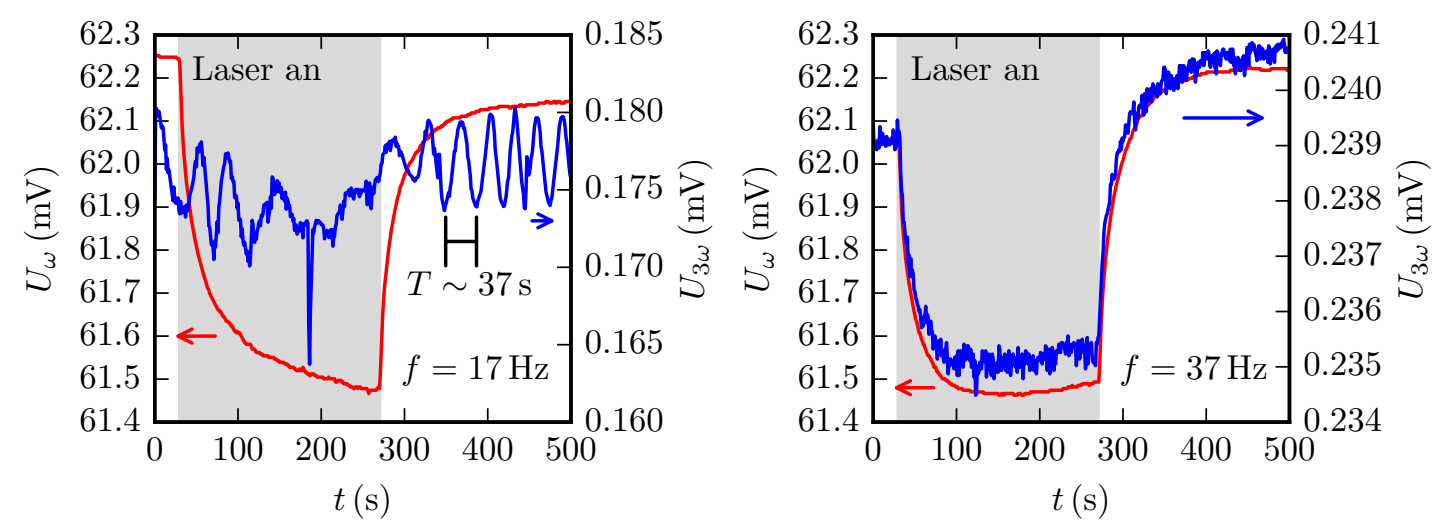

Abbildung 4.7.: Frequenzanalyse für Messungen der ersten und dritten harmonischen Spannung. Die Messungen fanden außerhalb des $\overline{\mathrm{PPMS}}$ bei Raumtemperatur mit $0.5 \mu \mathrm{A}$ statt; zwei sind hier abgebildet. Bei $17 \mathrm{~Hz}$ zeigt sich eine langsame Schwingung mit $T \sim 37 \mathrm{~s}$, wohingegen bei $37 \mathrm{~Hz}$ das Messsignal stabil ist.

Schichtdicke und laterale Ausdehnung entscheidend, sodass kleine Unsicherheiten dort schon zu größeren Änderungen führen. Dafür spielt ebenso eine mögliche magnetische Passivität der obersten Lagen der Schicht ein Rolle. So lässt sich das fehlende magnetische Moment erklären.

\subsubsection{Frequenzanalyse}

Da für die Messungen der dritten harmonischen Spannung neben dem fließenden Strom auch die Frequenz der Anregung variiert werden kann, war es ratsam, zunächst den zeitlichen Verlauf der Signale bei verschiedenen Frequenzen zu untersuchen. Hierfür wurde die Probe im Messkopf außerhalb des PPMS bei Raumtemperatur mit und ohne Laseranregung nach der Messgeometrie aus Abb. 3.1 bei einem Strom von 0.5 $\mu \mathrm{A}$ und Frequenzen $7 \ldots 997 \mathrm{~Hz}$ vermessen ${ }^{3}$. Dabei wurde die Laseranregung jeweils nach $30 \mathrm{~s}$ aktiviert und nach weiteren 4 min wieder deaktiviert.

Wie in Abb. $4.7 \mathrm{zu}$ sehen ist, besteht für eine Anregungsfrequenz von $17 \mathrm{~Hz}$, welche auch von MoshnYAGA et al. [Mos+09] verwendet wurde, eine ausgeprägte Schwingung im Messsignal mit einer Periodendauer von $\sim 37 \mathrm{~s}$. Dazu sei außerdem kritisch angemerkt, dass bei solch einer Frequenz die dritte Harmonische mit $51 \mathrm{~Hz}$ sehr nahe bei der Netzfrequenz des Niederspannungsnetzes liegt und dieses somit stark störende Einflüsse ausüben kann. Ebenso findet man ein Schwingverhalten mit dann allerdings kürzerer

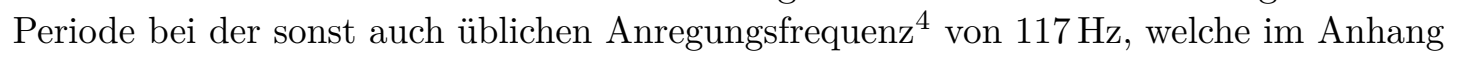
in Abb. A.1 gezeigt ist. Natürlich wirkt bei der tatsächlichen Messung im PPMS das

\footnotetext{
${ }^{3}$ Für die Frequenzen wurden Primzahlen verwendet, um mögliche Einflüsse durch niederfrequente kommensurable Störungen zu vermeiden.

${ }^{4}$ Diese Frequenz hat sich im Institut für Messungen etabliert, da sie gerade nicht mit bekannten Frequenzen oder Vielfachen davon zusammenfällt.
} 
Dewargefäß schirmend, sodass dort deutliche geringere Störungen erwartet werden können, aber durch geeignete Wahl der Frequenz können äußere starke Einflüsse von vornherein ausgeschlossen werden.

Bei Frequenzen ab $500 \mathrm{~Hz}$ und höher nimmt die Amplitude des gemessen Signals sowohl für $U_{\omega}$ als auch für $U_{3 \omega}$ ab, da hier vermutlich die kapazitiven Eigenschaften der elektrischen Messleitungen zum Tragen kommen und über den entsprechenden Blindwiderstand das Messsignal abschwächen.

Nun zeigt die Messung bei $37 \mathrm{~Hz}$ keine Schwingung und weist eine annehmbare Amplitude der Messgrößen auf, weshalb diese Frequenz für alle späteren Messungen der ersten und dritten harmonischen Spannung verwendet wurde.

\subsubsection{Bestimmung des Fokus}

Für die Anregung des Stegs durch den Laser ist es sehr bedeutsam, dass die Photonendichte auf der Probe besonders hoch ist, um möglichst viele Elektronen im System zu beeinflussen. Hierfür ist neben der zeitlichen auch die räumliche Ausdehnung der Anregung entscheidend und somit sollte das Ziel sein, eine möglichst kleine Fläche des Films zu beleuchten, d.h., den Fokus der Linse auf die Probenoberfläche einzustellen, sodass die Strahltaille möglichst klein wird. Jedoch darf dabei der Lichtpunkt nicht kleiner als die Breite des Stegs werden, da sonst ein unbeeinflusster Pfad im Steg die durch die Laseranregung veränderten Bereiche kurzschließen kann.

Natürlich ist eine Bestimmung der Strahltaille nur möglich, wenn man, wie in Kap. 3.9 beschrieben, eine scharfe Kante auf der Probe hat und die Antwortfunktion ebenfalls diese scharfe Kante abbilden kann. Ersteres ist durch die Strukturierung sichergestellt, die Genauigkeiten im Submikrometerbereich erlaubt. Die zweite Bedingung wird jedoch lediglich von der Reflektivität als Messgröße erfüllt, da bei der Thermospannung durch Wärmediffusion größere Bereiche als die vom Laser getroffenen angeregt werden, wodurch sich folglich auch nur die Reflektivität zum Vermessen bzw. Optimieren der Strahltaille des Lasers eignet. Allerdings war die Abbildung mithilfe der Thermospannung (s. Abb. 4.8) hinreichend genau, sodass sich dort auch auf eine kleine Strahltaille schließen lässt. Und tatsächlich hat sich aus späteren Messungen der Reflektivität ergeben, dass sich für die Strahltaille in Göttingen eine Breite von $40 \mu \mathrm{m}$ ergibt, was für den Steg mit einer Breite von $30 \mu \mathrm{m}$ passend ist.

In Greifswald konnte auch anhand der Reflektivität der Fokus mit einer Strahltaille von $80 \mu \mathrm{m}$ eingestellt werden.

\subsubsection{Positionierung}

Zur Positionierung des Lasers wurde, wie in Kap. 3.10 beschrieben, anfangs die Thermospannung an den äußeren beiden Kontakten der Struktur gemessen, während der Laser über die Probenoberfläche gerastert wurde. Mithilfe dieser Daten lässt sich eine Karte der Probenoberfläche erstellen, wie sie Abb. 4.8 zeigt. 

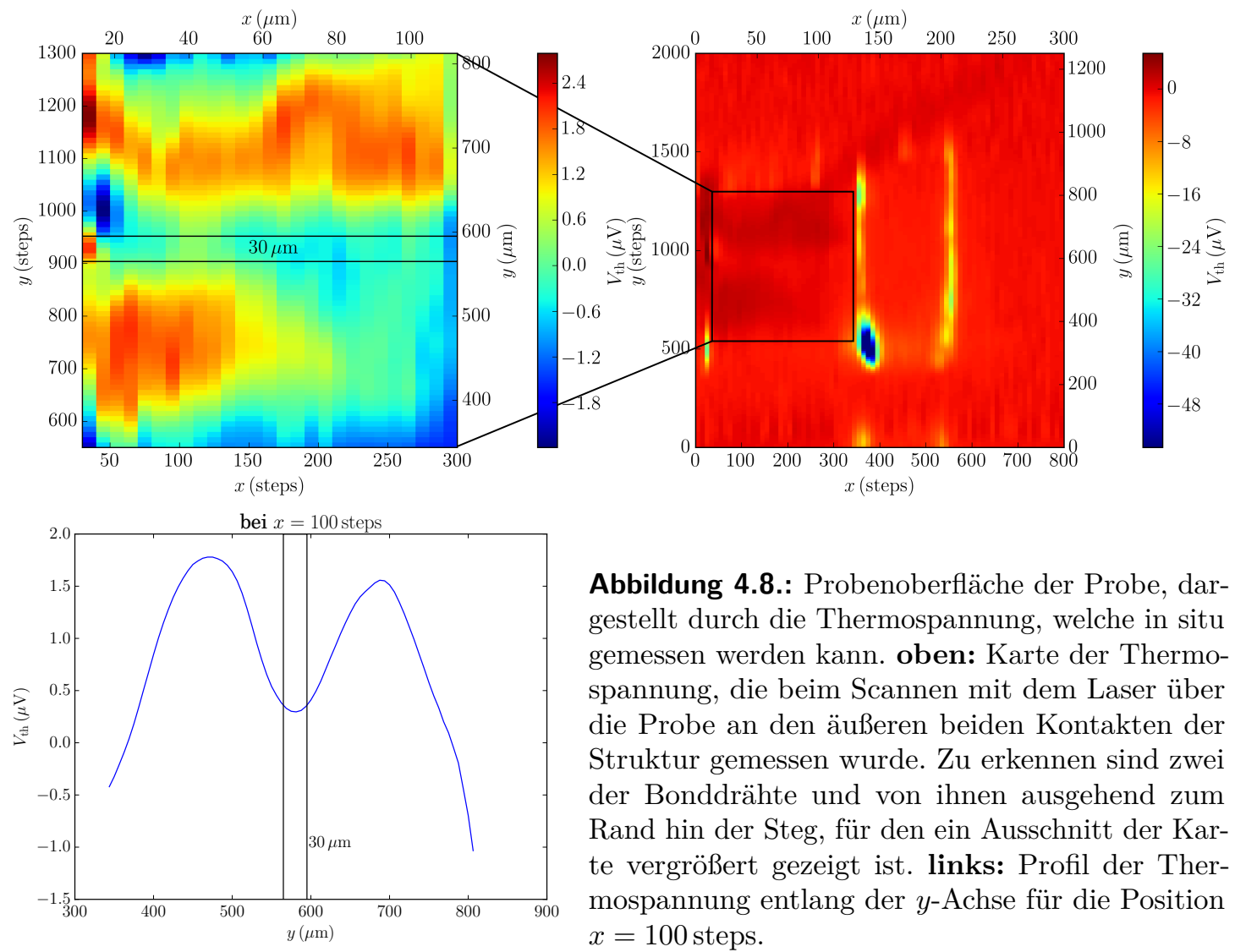

Abbildung 4.8.: Probenoberfläche der Probe, dargestellt durch die Thermospannung, welche in situ gemessen werden kann. oben: Karte der Thermospannung, die beim Scannen mit dem Laser über die Probe an den äußeren beiden Kontakten der Struktur gemessen wurde. Zu erkennen sind zwei der Bonddrähte und von ihnen ausgehend zum Rand hin der Steg, für den ein Ausschnitt der Karte vergrößert gezeigt ist. links: Profil der Thermospannung entlang der $y$-Achse für die Position $x=100$ steps. 


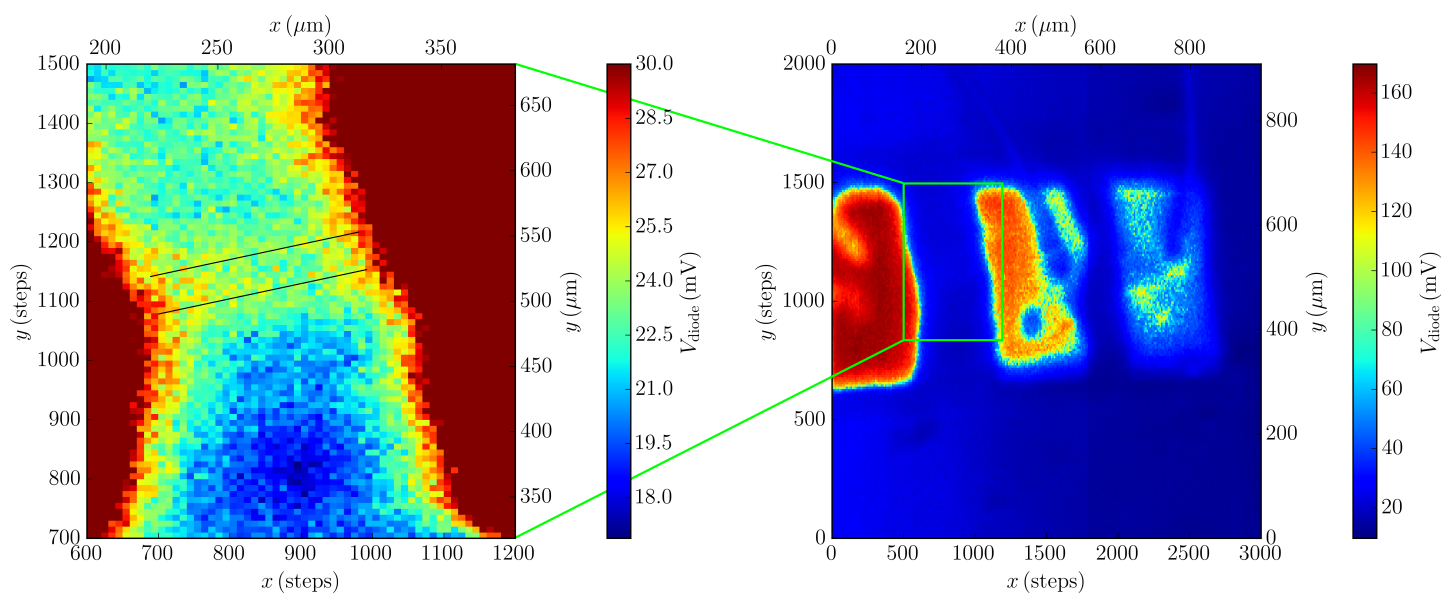

Abbildung 4.9.: Karte der Probenoberfläche anhand der Reflektivität. Die mit Gold bedampften Kontaktflächen sind aufgrund ihrer hohen Reflektivität deutlich zu erkennen, ebenso sind die Bonddrähte auszumachen. Der Ausschnitt, in dem sich der Steg befindet, ist vergrößert und der Kontrast für den Bereich wurde gespreizt, sodass kleine Schwankungen sichtbar werden, aber die größeren Werte übersättigen. Dadurch lässt sich der Steg erkennen.

Darauf sind klar die Bonddrähte zu erkennen und der Bereich zwischen den Kontakten, in dem der Steg verläuft. So zeigt dann auch eine Vergrößerung dieses Ausschnittes deutlich eine Talstruktur, die den Steg mit einer Breite von $30 \mu \mathrm{m}$ repräsentiert.

Außerdem wurde bei späteren Messungen für die Positionierung des Lasers die Reflektivität zu Hilfe genommen, da sich diese Methode als robuster herausstellte. So zeigt Abb. 4.9 deutlich die Kontaktpads und die darauf gebondeten Drähte. Erstere reflektieren besonders gut, da sie bei der Strukturierung mit Gold bedampft wurden. Da sich die Reflektivität des schmalen Stegs aus dünnem Manganatfilm jedoch nur gering von der des Substrates unterscheidet [vgl. Car65; \& Tak+00], ist dieser nicht direkt erkennbar, sondern nur, wenn der Kontrast in dem entsprechenden Bereich gespreizt wird. Für eine Positionierung des Lasers ist diese Information hinreichend.

\subsubsection{Einfluss von Laseranregung auf die dritte harmonische Spannung}

Ist nun also der Anregungsfleck des Lasers auf dem Steg positioniert, kann sein Einfluss auf die elektrischen Messgrößen untersucht werden. Dafür wurde der Messaufbau nach Abb. 3.1 realisiert, wobei als Anregungsspannung mit $U_{\text {ex }}=5 \mathrm{~V}$ das Maximum des Lock-in-Verstärkers und als Vorwiderstand $R_{\text {vor }}=10 \mathrm{M} \Omega$ gewählt wurde, sodass der Anregungsstrom konstant $I_{\mathrm{ex}}=0.5 \mu \mathrm{A}$ betrug. Anschließend wurde mithilfe des PPMS die gewünschte Temperatur angefahren und auf ihre Stabilisierung gewartet, um dann den folgenden Messzyklus für diese Temperatur durchzuführen:

1. Für eine Zeit von 50s wurden die Messsignale der ersten harmonischen Spannung $U_{\omega}$ und der dritten harmonischen Spannung $U_{3 \omega}$ ohne Anregung, d. h. bei geblocktem Laser aufgezeichnet. 
2. Die Blockierung wurde aufgehoben und die Probe für die nächsten $150 \mathrm{~s}$ dem gepulsten Laser mit einer Leistung von $\sim 10 \mathrm{~mW}$ ausgesetzt.

3. Wiederum wurde der Laser geblockt und die Daten für $200 \mathrm{~s}$ aufgenommen.

Danach konnte die nächste Temperatur eingestellt werden.

Die dabei aufgezeichneten Zeitserien zeigt Abb. $4.10 \|^{5}$ Dort ist zu sehen, dass nach Beginn der Anregung sowohl die erste als auch dritte harmonische Spannung sich auf einer langsamen Zeitskala von 100s entwickeln, bevor sich das Signal setzt. Dieses ist mithilfe des Wärmeeintrags durch den Laser in die Probe und das spätere Erreichen einer stationären Temperatur zu erklären. Außerdem erkennt man schon die Temperaturabhängigkeit der Signale, die in der Nähe des Metall-Isolator-Übergangs ein Maximum aufweisen.

Um nun diese Abhängigkeit zu extrahieren, wurden die Signallevel bei $35 \ldots 45$ s vor der Anregung als Messwert für den unbeleuchteten Zustand und bei $185 \ldots$.. 195 s für den beleuchteten Zustand angenommen. Daraus ergibt sich der Graph aus Abb. 4.11, wobei jeweils die Kurven mit Laseranregung zusätzlich mit einer Temperaturkorrektur eingezeichnet sind, die sich aufgrund des Wärmeeintrags durch den Laser ergibt.

So sieht man dann auch, dass für die erste harmonische Spannung, die ja proportional zum Probenwiderstand ist, nach der Temperaturkorrektur die Kurven mit und ohne Anregung nahezu deckungsgleich sind, wohingegen sich für die dritte harmonische Spannung eine zum Maximum ansteigende Differenz ergibt. Folglich kann man davon ausgehen, dass die Änderung im Widerstand lediglich einen Temperaturzuwachs durch den Laser darstellt, sich aber in der dritten harmonischen Spannung noch andere Effekte als eine reine Temperaturerhöhung darstellen, die am Maximum am ausgeprägtesten sind und in den Bereichen höherer und niedrigerer Temperaturen verschwinden.

Weiterhin ist auffällig, dass die Maxima in der ersten und dritten harmonischen Spannung nicht ganz zusammenfallen, sondern sich um ungefähr $7 \mathrm{~K}$ unterscheiden.

Nun kann man den Quotienten der beiden harmonischen Spannungen, den so genannten dritten harmonischen Koeffizienten $K_{3 \omega}=U_{3 \omega} / U_{\omega}$ betrachten (s. Kap. 2.2), der ja laut Moshnyaga et al. Mos+09 proportional zur Dichte der Polaronen ist. Die entsprechenden Graphen zeigt Abb. 4.12 und auch hier ist zu erkennen, dass am Übergang das Signal durch die Laseranregung erhöht ist, und zwar um $\sim 1 \%$.

\section{Greifswald}

Im Folgenden sollen jetzt die vergleichbaren Messungen, die mithilfe des Lasersystems in Greifswald (s. Kap. 3.5.3 an dem Aufbau, der in Kap. 3.8 beschrieben ist, gezeigt werden. Das heißt, es handelt sich dabei um Messungen mit einem Freistrahl, wobei die Leistung auf der Probe $100 \mathrm{~mW}$ betrug. Dabei wurden ebenfalls zunächst die Zeitserien aufgenommen, welche im Anhang in Abb. A.3 abgedruckt sind, und daraus ebenso die Temperaturabhängigkeit extrahiert, was in Abb. 4.13 dargestellt ist.

Auch hier zeigen die Graphen, dass es aufgrund des Lasers eine Temperaturerhöhung auf der Probe ergibt, die in diesem Fall mit 15.25 K wegen der zehnfachen Leistung

\footnotetext{
${ }^{5}$ Der Übersicht halber ist nur etwa die Hälfte aller Temperaturen in der Abbildung dargestellt.
} 


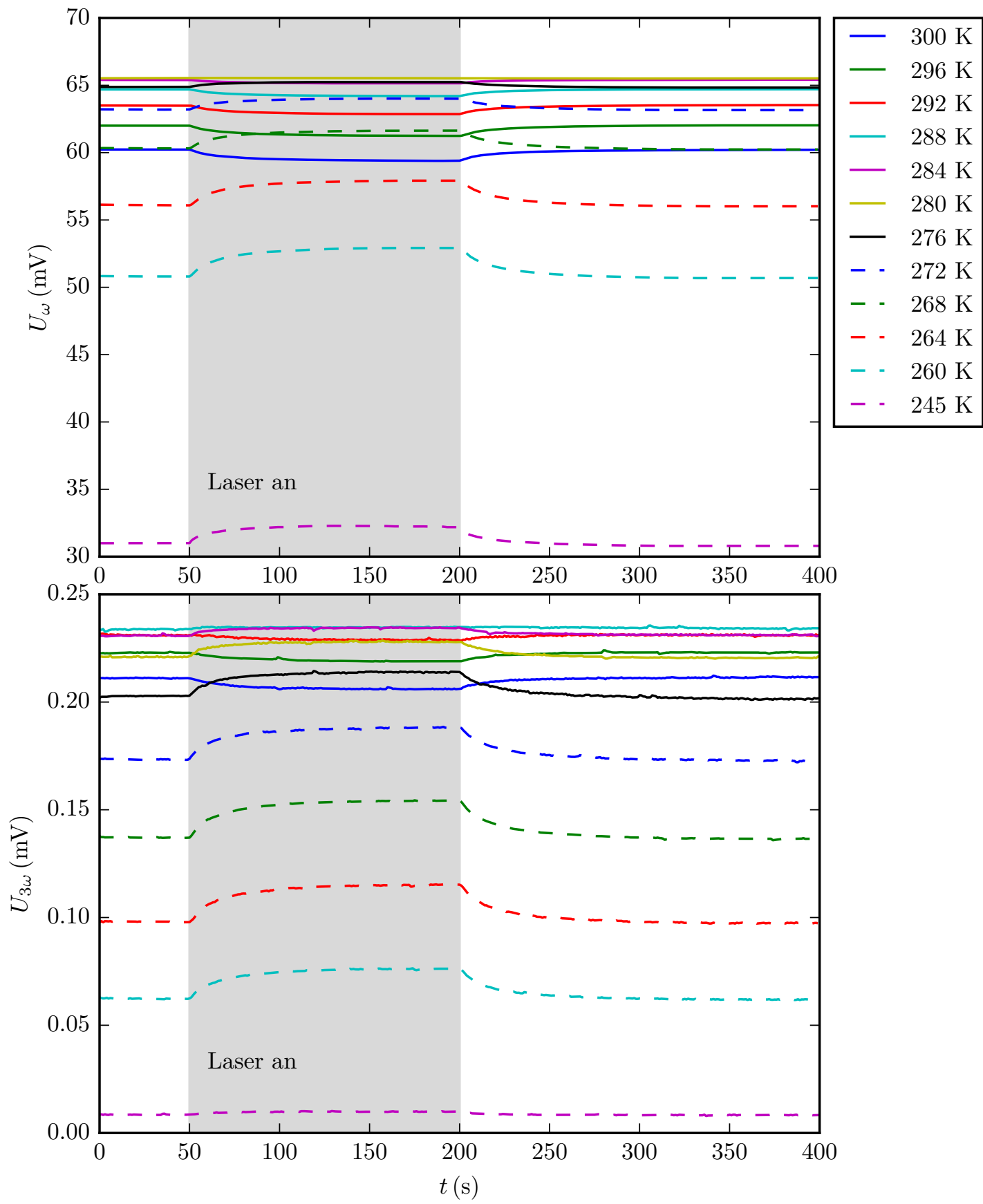

Abbildung 4.10.: Zeitentwicklung der ersten (oben) und dritten harmonischen Spannung (unten) unter Laseranregung. Nach 50 s Datenaufnahme ohne Anregung wird der Laser freigegeben und das Signal verändert sich auf langer Zeitskala. Nach weiteren $150 \mathrm{~s}$ wird der Laser wieder geblockt und das Signal relaxiert zum ursprünglichen Wert zurück. Die Messung wird nach insgesamt $400 \mathrm{~s}$ beendet. Es sei nochmal angemerkt, dass die Laseranregung mit ultrakurzen Pulsen erfolgt (s. a. Kap. 3.6. 

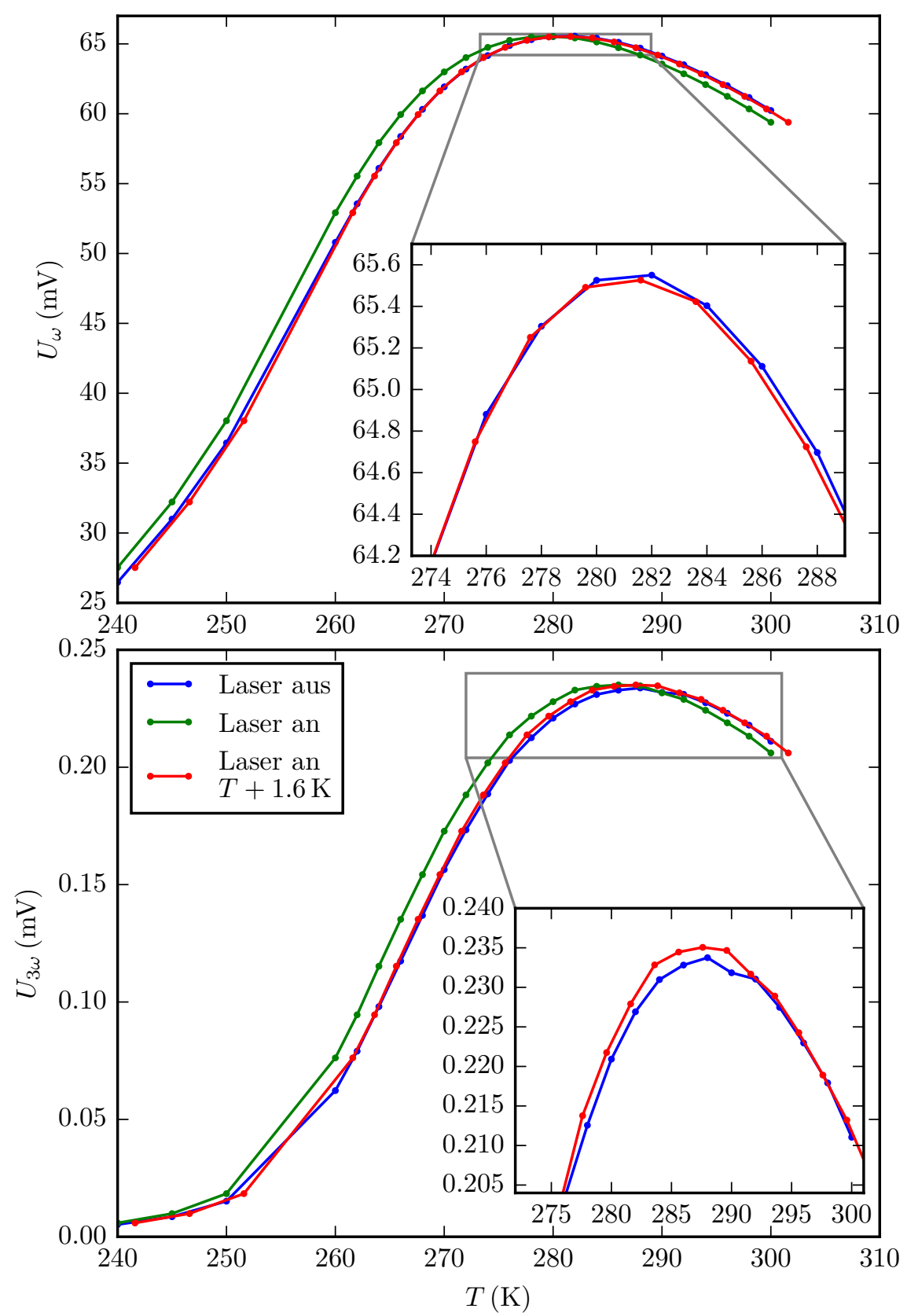

Abbildung 4.11.: Temperaturabhängigkeit der ersten (oben) und dritten harmonischen Spannung (unten) mit und ohne Laseranregung, extrahiert aus dem Messdaten von Abb. 4.10 Die Kurven unter Beleuchtung müssen aufgrund des Wärmeeintrags durch den Laser zu höheren Temperaturen verschoben werden. Dabei fallen die Kurven für die erste harmonische Spannung übereinander, was reine Temperaturverschiebung nahelegt, bei der dritten harmonischen Spannung unterscheiden sich die entsprechenden Kurven jedoch auch nach der Verschiebung um $\sim 0.6 \%$. 

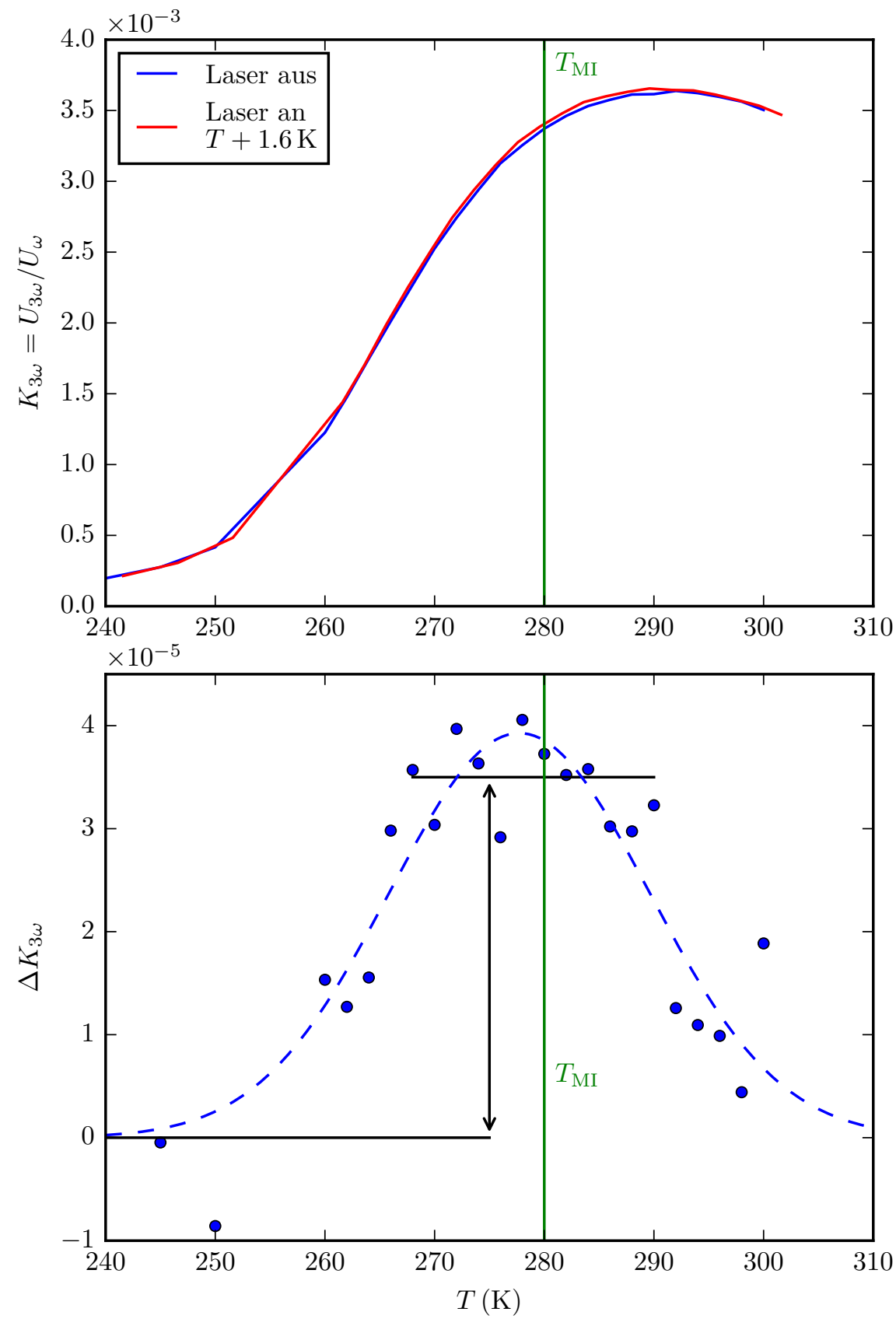

Abbildung 4.12.: Lichtinduzierte Änderung des dritten harmonischen Koeffizienten, der sich sich als Quotient $U_{3 \omega} / U_{\omega}$ aus den harmonischen Spannungen ergibt. Bei der Übergangstemperatur stellt man eine Erhöhung des Koeffizienten um 1\% durch die Laseranregung fest. 


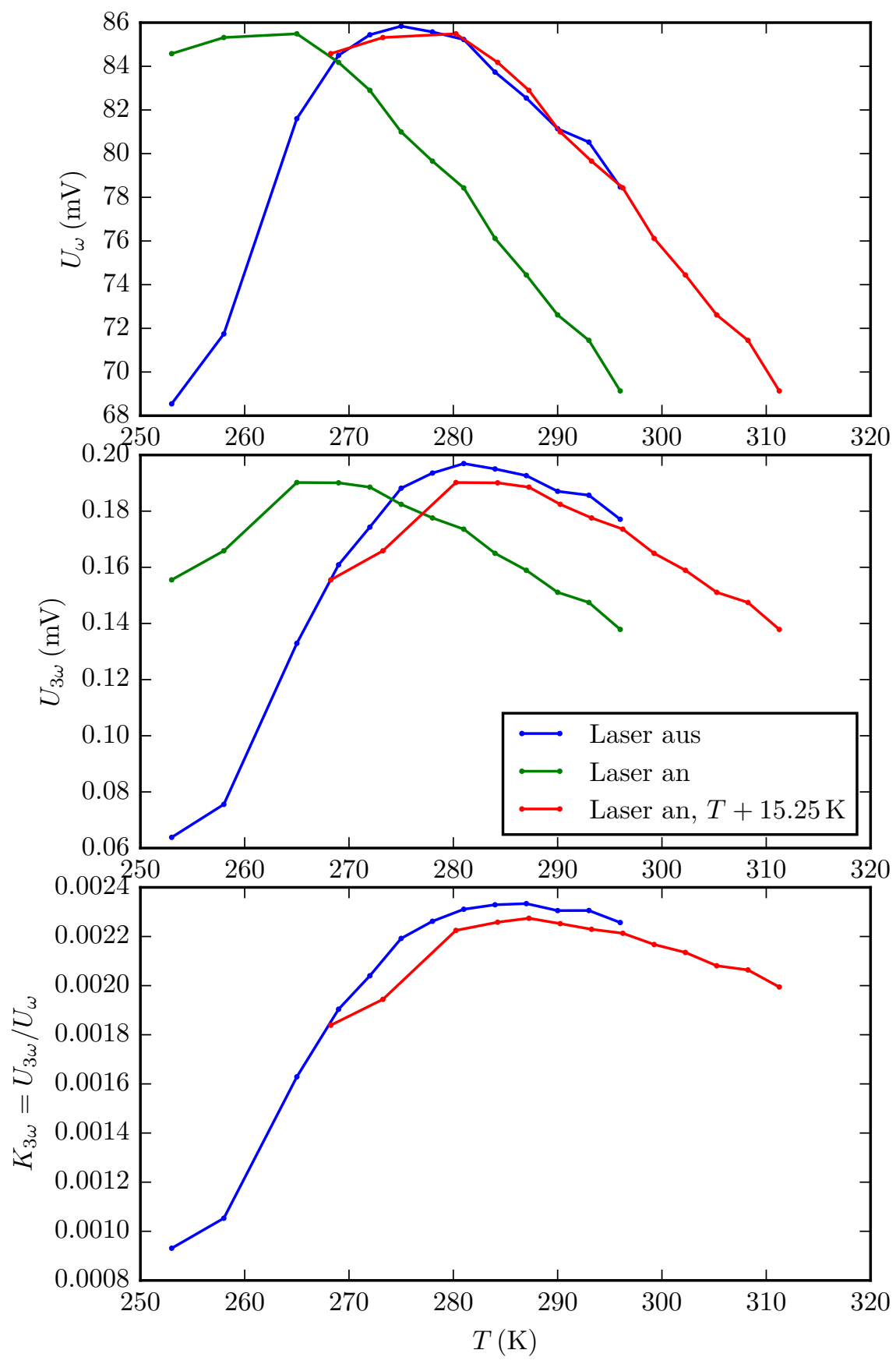

Abbildung 4.13.: Temperaturabhängigkeit der ersten (oben) und dritten harmonischen Spannung (mittig) und des dritten harmonischen Koeffizienten (unten) von Messungen am Lasersystem in Greifswald. Nach der Temperaturkorrektur fallen die Kurven für $U_{\omega}$ nahezu übereinander, bei $U_{3 \omega}$ ergibt sich jedoch eine Erniedrigung aufgrund des Lasers um $\sim 3.4 \%$. $K_{3 \omega}$ sinkt um $\sim 2.5 \%$. 


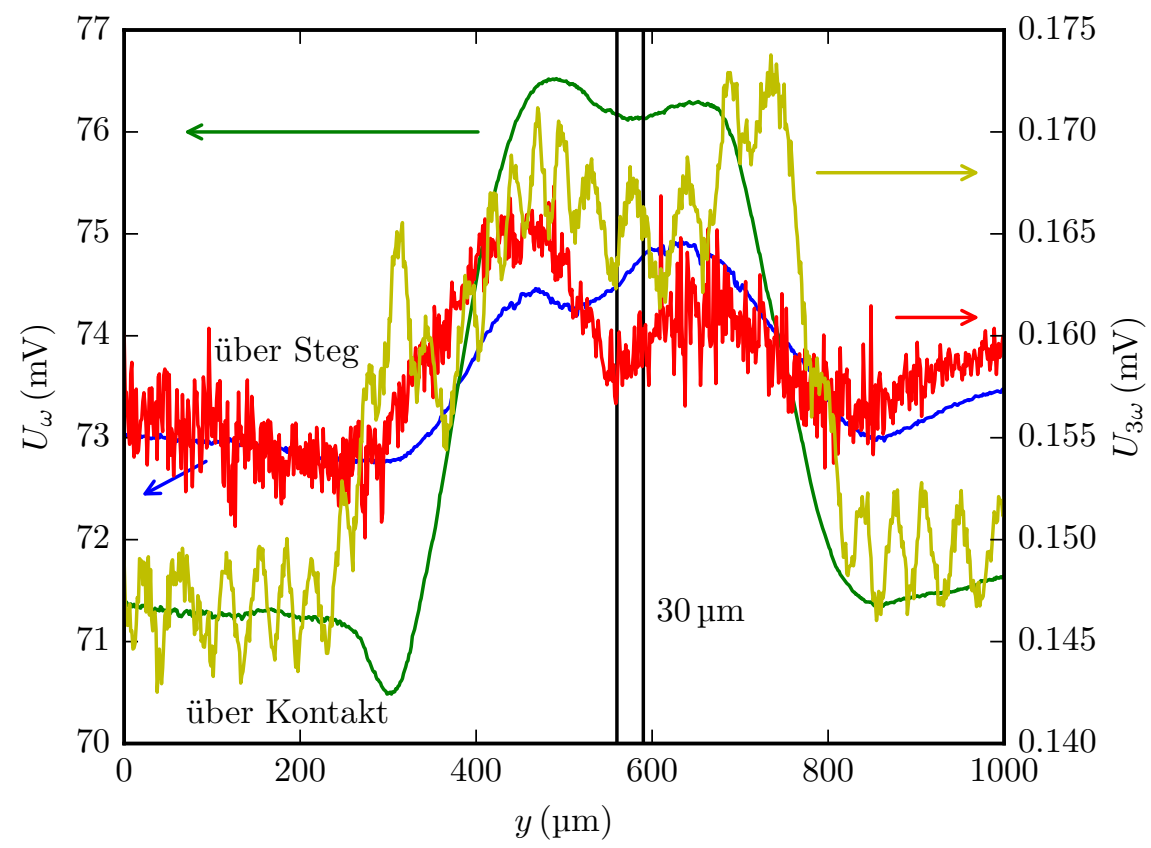

Abbildung 4.14.: Harmonische Spannungen beim Linearscan des Lasers über die Probe (Greifswald). Der Anregungsfleck des Lasers wurde bei $288 \mathrm{~K}$ mit einer Geschwindigkeit von $2.5 \mathrm{\mu m} / \mathrm{s}$ senkrecht zur Struktur über die Probe gezogen, während die harmonischen Spannungen aufgezeichnet wurden. Dabei überquerte dieser bei einer Messung den Steg und bei einer anderen einen Kontakt.

auch zehnmal so hoch ist und nach deren Korrektur eine beinahe Kongruenz für $U_{\omega}$ festzustellen ist. Jedoch zeigt $U_{3 \omega}$ hier im Gegensatz zu den vorherigen Messungen in Göttingen eine große Verringerung des Signals um 3.4\% durch die Anregung und damit auch in $K_{3 \omega}$ eine Erniedrigung um $\sim 2.5 \%$.

Bei allen hier beschriebenen Messungen ist zu berücksichtigen, dass sich die Veränderungen als zeitliches Mittel aufgrund der elektrischen Messtechnik ergeben (s. Kap. 3.6) und die tatsächlichen Effekte wesentlich größer sind, was in Kap. 5.4.1 diskutiert wird.

\subsubsection{Abhängigkeit der harmonischen Spannungen von der Anregungsposition}

Um nun zu untersuchen, wie die Probe auf Stimuli neben dem Steg antwortet, wurden in Greifswald Messungen mit Anregungen auf unterschiedlichen Positionen der Probe durchgeführt. Dafür wurde zum einen während der Aufzeichnung von erster und dritter harmonischer Spannung nach Abb. 3.1 der Laser mit $100 \mathrm{~mW}$ langsam über die Probe bewegt, was in Abb. 4.14 gezeigt ist. Dort ist eine Struktur mit einer Ausdehnung von $\sim 400 \mu \mathrm{m}$ zu erkennen, die passend zum freigestellten Bereich der Probe ist, in dem sich der Steg befindet (s. Abb. 4.4). Davon jeweils oberhalb und unterhalb ist Vollfilm, der 


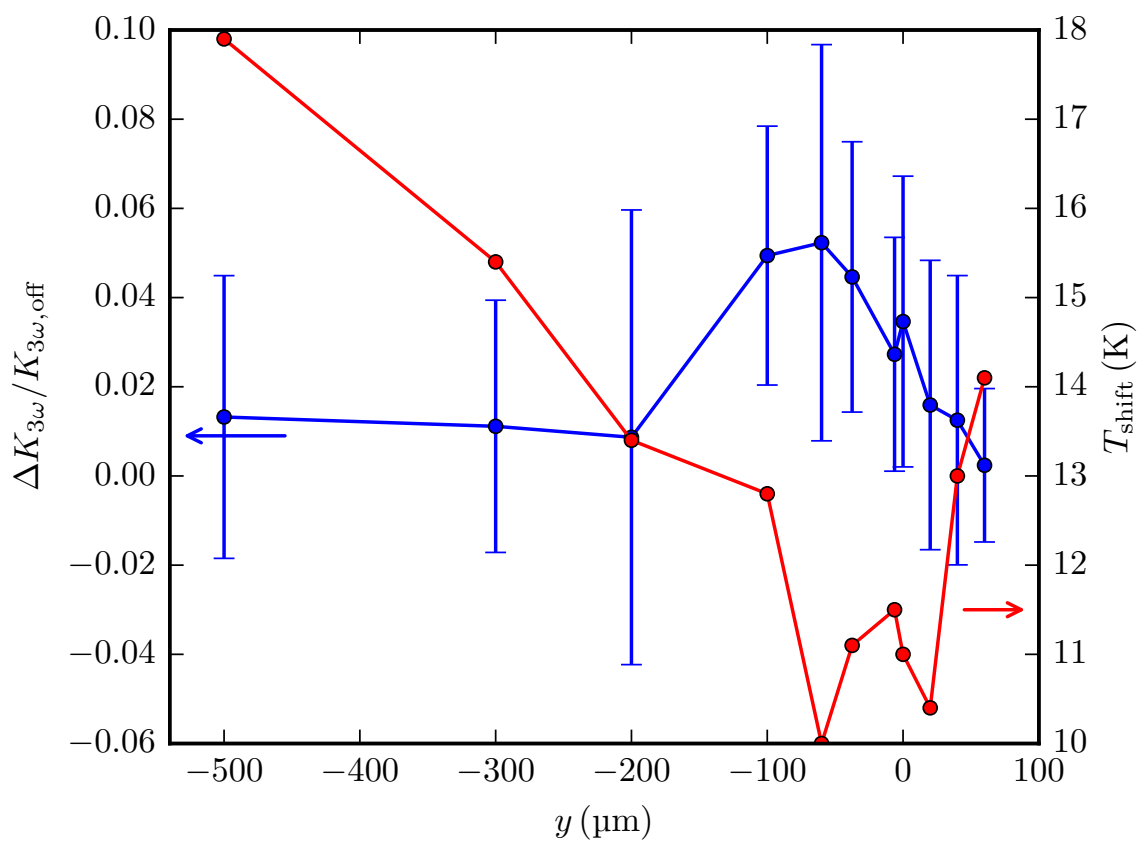

Abbildung 4.15.: Änderung des dritten harmonischen Koeffizienten mit der Anregungsposition und Temperaturverschiebung (Greifswald). Aus den Temperaturkurven bei unterschiedlicher Anregungsposition (Anhang Abb. A.2 ergibt sich aus der Position der Maxima die Temperaturverschiebung (rot). Nach Korrektur kann daraus der harmonische Koeffizient $K_{3 \omega}=U_{3 \omega} / U_{\omega}$ bestimmt werden, dessen relative Änderung aufgrund der Anregung hier zu sehen ist (blau). Die Position des Stegs ist zwischen $-100 \mu \mathrm{m}$ und $0 \mu \mathrm{m}$ anzunehmen.

jedoch nicht mit dem Steg oder Kontakt verbunden ist, außer über das Substrat. In der Mitte, also bei $y \sim 575 \mu \mathrm{m}$ ist der Steg mit einer Ausdehnung von $30 \mu \mathrm{m}$ lokalisiert, was sich in den Messungen - wenn auch sehr verbreitert - widerspiegelt. Dabei fällt jedoch sofort auf, dass auch bei dem Scan über einen Kontakt die Position des Steges erkennbar wird.

Bei der zweiten Methode wurde für verschiedene Anregungspositionen jeweils gleichzeitig eine vollständige Temperaturkurve für $U_{\omega}$ und $U_{3 \omega}$ mithilfe der Peltierkühlung (s. Kap. 3.8) aufgenommen, welche Abb. A.2 im Anhang zeigt. Aus diesen Kurven konnte durch Betrachtung der Position vom Maximum der ersten harmonischen Spannung die Temperaturverschiebung aufgrund des Energieeintrags durch den Laser zu der nicht angeregten Kurve bestimmt und entsprechend korrigiert werden. Anschließend berechnete man den dritten harmonische Koeffizient, also $K_{3 \omega}=U_{3 \omega} / U_{\omega}$, und dann seine Änderung durch die Laseranregung als Differenz zum unbeleuchteten Fall, was Abb. 4.15 zeigt. Dort kann vielleicht eine Zunahme des Signals beim Steg vermutet werden, es ist jedoch anzumerken, dass die Fehler aufgrund der sehr verrauschten Datenaufnahme groß sind. Weiterhin auffällig ist außerdem, dass die Temperaturverschiebung aufgrund des Wärmeeintrags in der Nähe des Stegs bzw. des strukturierten Bereichs kleiner ist 


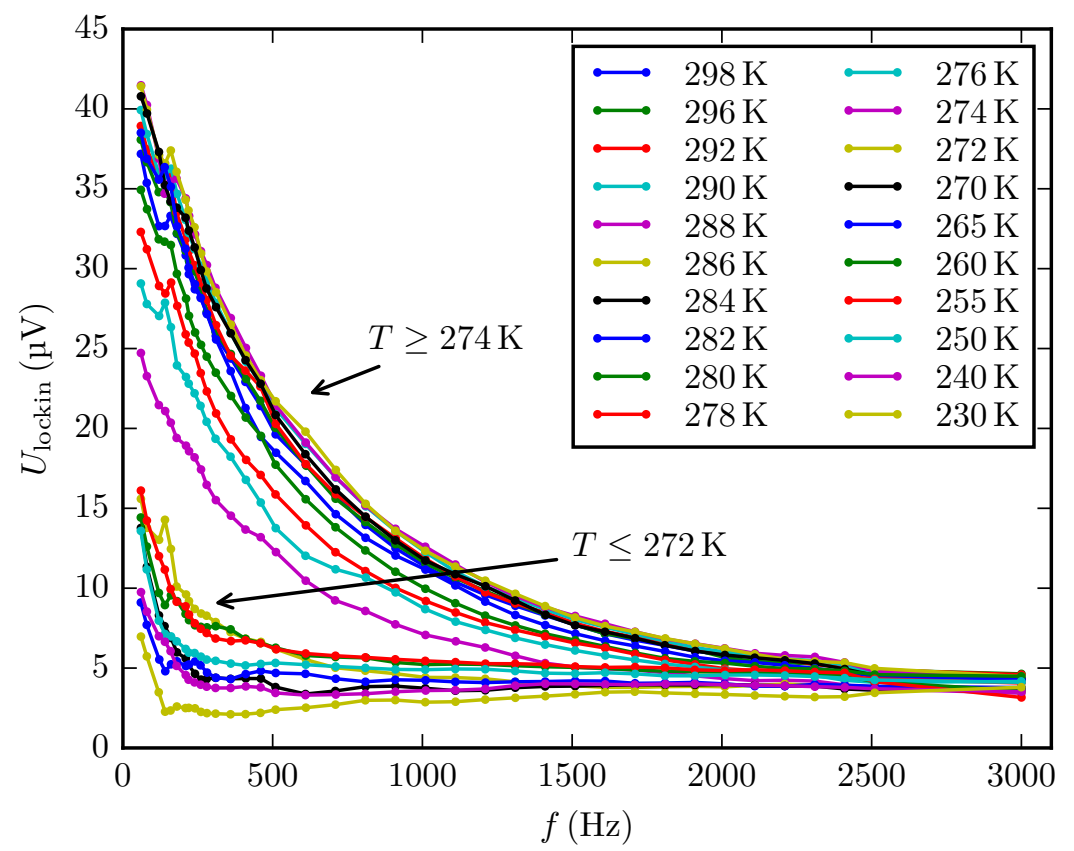

Abbildung 4.16.: Änderung des Gleichstromwiderstandes durch periodische Lasereinwirkung in Abhängigkeit der Anregungsfrequenz. Gezeigt ist hier die in Kap. 3.3 beschriebene Messung, wobei die Daten leicht geglättet wurden. Zwischen $274 \mathrm{~K}$ und $272 \mathrm{~K}$ trennen sich die Kurven in zwei Bereiche, bei hohen Temperaturen mit hoher charakteristischer Frequenz und bei niedrigen Temperaturen mit niedriger.

als weiter entfernt davon.

\subsubsection{Wärmedissipation}

Der Einfluss der Temperaturänderung bei der Anregung sollte mit einem Aufbau wie in Kap. 3.3 geschildert untersucht werden. Das heißt, bei einem konstanten aufgeprägten Gleichstrom wurde die durch die periodische Anregung durch den Laser induzierte Spannung mit einem Lock-in-Verstärker aufgezeichnet, wobei die Anregungsfrequenz variiert wurde. Daraus ergaben sich die Messungen in Abb. 4.16 und man erkennt deutlich zwei zwischen $274 \mathrm{~K}$ und $272 \mathrm{~K}$ getrennte Temperaturbereiche, was dafür spricht, dass in der Tieftemperaturphase sich ein Dissipationskanal schließt, wodurch die charakteristische Frequenz abnimmt und eine damit verbundene charakteristische Zeit für Wärmedissipation zunimmt. Eine ausführlichere Diskussion findet man in Kap. 5.3 .

\subsubsection{Strom-Spannungs-Kennlinien}

Im Wesentlichen rühren die Messsignale in den höher-harmonischen Spannungen von den Nichtlinearitäten der Strom-Spannungskennlinie her, da aufgrund dieser der flie- 


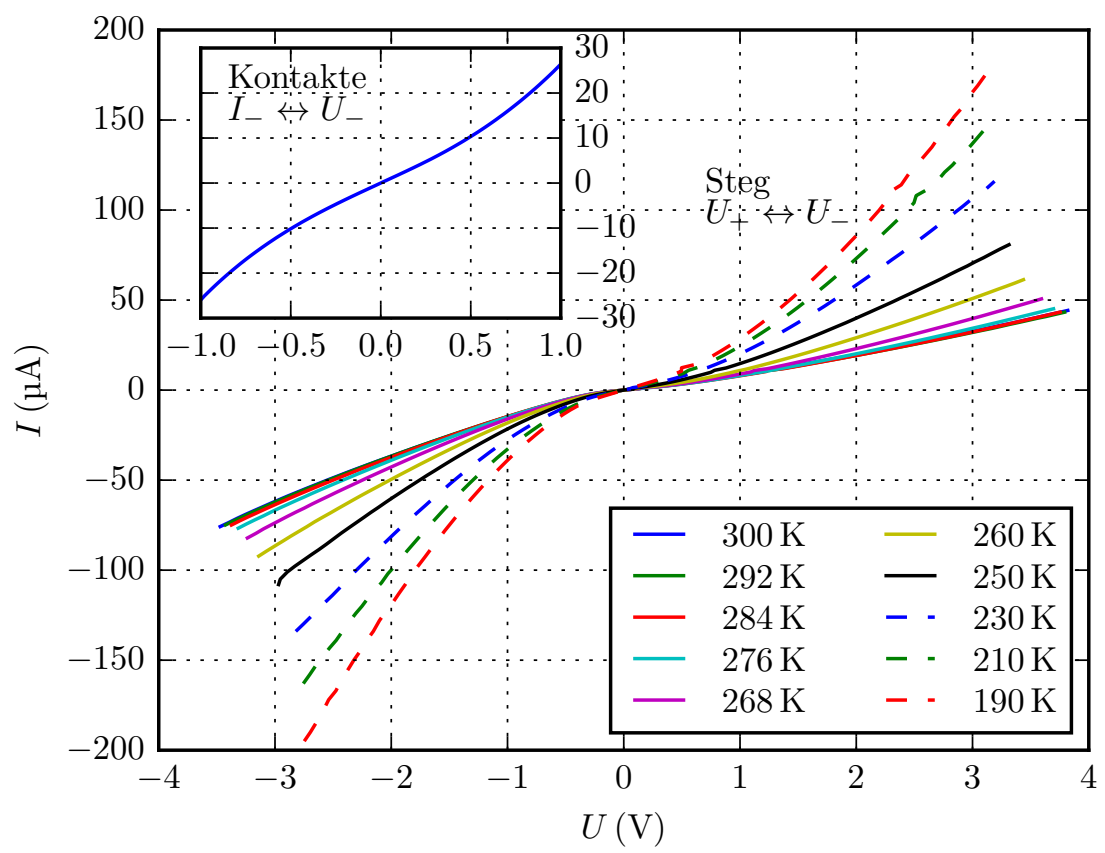

Abbildung 4.17.: Strom-Spannungs-Kennlinien, mit Gleichspannung über den Steg bzw. über zwei benachbarte Kontakte (Nebenbild) gemessen. Die nichtlineare Form ist sofort offensichtlich, aber es ist ebenfalls eine Asymmetrie zu erkennen, welche jedoch nur bei Messung über den Steg und nicht bei Messung zwischen zwei benachbarten Kontakten wie $I_{-}$und $U_{-}$auftritt.

Bende Strom durch die Probe nicht wie bei einem Ohmschen Widerstand proportional zur angelegten Spannung ist und damit bei sinusodialer Anregung höher-harmonische Anteile entstehen ${ }^{6}$ Daher wurden die Strom-Spannungs-Kennlinien, wie in Kap. 3.2 beschrieben, von Raumtemperatur bis hinunter zu $190 \mathrm{~K}$ aufgezeichnet, wobei einmal alle vier Kontakte der Probe benutzt und somit über den Steg, aber zum zweiten auch nur durch zwei benachbarte Kontakte ohne den Steg gemessen wurde (s. Abb. 4.4). So zeigt dann auch wie erwartet Abb. 4.17 ein nichtlineares Verhalten der Probe, das allerdings auffälligerweise deutlich asymmetrisch ist. Jedoch ist dieses nur für die Messungen der Fall, bei denen der Strom den Steg durchfließen muss. Verwendet man aber benachbarte Kontakte auf einer Seite des Steges, also ehemals $I_{-}$und $U_{-}$, so liegt wieder symmetrisches Verhalten vor.

\subsection{Lanthan-Praseodym-Calcium-Manganat}

Da durch den neuen Messeinsatz im PPMS leicht niedrige Temperaturen erreicht werden können, wurde als zweite Probe Lanthan-Praseodym-Calcium-Manganat (LPCMO) in der Form $\left(\mathrm{La}_{1-y} \mathrm{Pr}_{y}\right)_{1-x} \mathrm{Ca}_{x} \mathrm{MnO}_{3}$ mit einer Dotierung von $x \approx 0.3$ und $y \sim 0.4$ auf

${ }^{6}$ Siehe dafür auch Kap. 2.2 


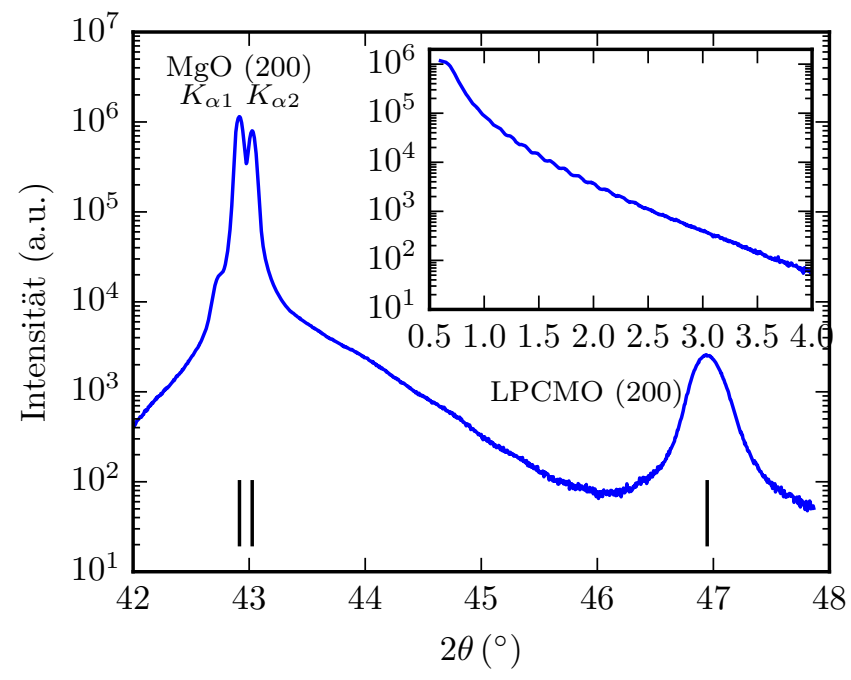

Abbildung 4.18.: Röntgendiffraktometrie von LPCMO mit Weit- und Kleinwinkelmessung (Nebenbild). Es ergibt sich eine Gitterkonstante von $c=3.871 \AA$ und eine Schichtdicke von $60 \mathrm{~nm}$. Für das Substrat $\mathrm{MgO}$ erhält man $c=4.215 \AA$. Daten von Oleg Shapoval.

einem MgO-Substrat mit (100)-Orientierung gewählt, welche von Oleg ShapovaL $7^{7}$ im Rahmen eines Gastaufenthaltes im Institut hergestellt wurd 8 . Dieses Material weist dann stärkere elektronische Korrelationen aufgrund der größeren Elektron-PhononWechselwirkung auf, weswegen aber eben auch die charakteristischen Temperaturen deutlich unterhalb der Raumtemperatur liegen. Da LPCMO gegenüber dem Substrat $\mathrm{MgO}$ aufgrund unterschiedlicher Gitterkonstanten eine Fehlpassung von ungefähr $10 \%$ aufweist, ist die Wachstumsmode eine andere. In den ersten wenigen Monolagen ergeben sich also zwingend viele Versetzungen, wonach jedoch in höheren Lagen die Schicht völlig unverspannt wächst [Hüh10].

\subsubsection{Grundlegende Charakterisierung}

Hier sollen zunächst die charakterisierenden physikalischen Eigenschaften wie Oberfläche, Struktur und Widerstand dargestellt werden, um eine Bewertung der Qualität der Probe, insbesondere auch nach der Strukturierung, zu ermöglichen.

\section{Oberfläche und Struktur}

Mithilfe der Röntgendiffraktometrie, deren Daten in Abb. 4.18 gezeigt sind, kann man den Gitterparameter für die pseudokubische Einheitszelle zu $c=3.871 \AA$ bestimmen, was vergleichbar mit den Proben von HüHN [Hüh10 ist. Ebenso erhält man aus der Kleinwinkelmessung eine Angabe für die Schichtdicke von $60 \mathrm{~nm}$. Aus den Aufnahmen im Rastertunnelmikroskop, wie sie Abb. 4.19 zeigt, sieht man, dass die Schicht im Inselwachstum um die tiefer liegenden Versetzungen einigermaßen glatt wächst, was eine

\footnotetext{
${ }^{7}$ Institute of Electronic Engineering and Nanotechnologies, Academy of Sciences of Moldova, Chisinau, Moldawien

${ }^{8}$ Die Probe trägt die Nummer X833w.
} 


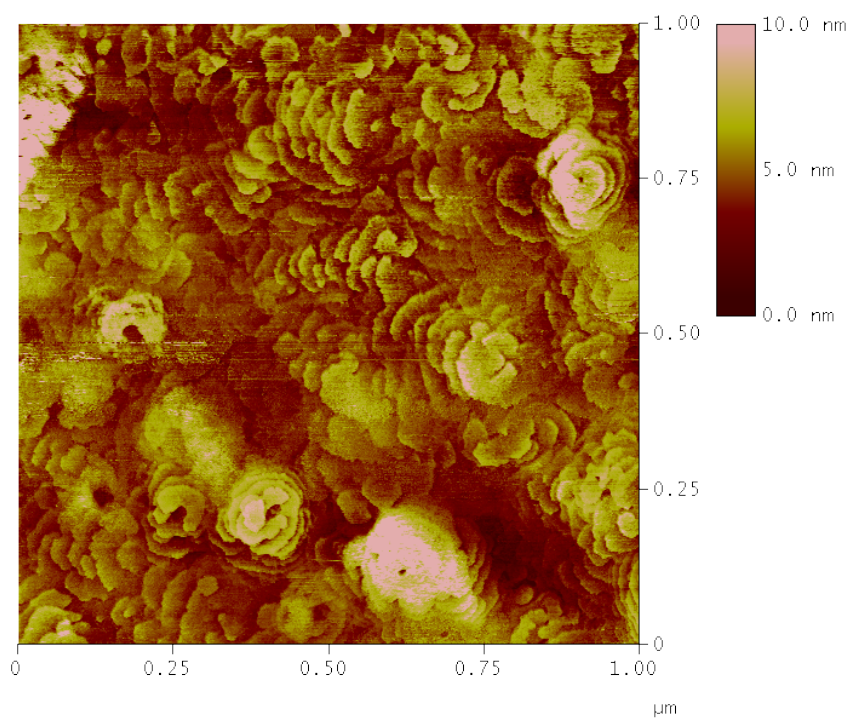

Abbildung 4.19.: Rastertunnelmikroskop-Aufnahme der LPCMO. Probe. Es liegt Inselwachstum vor und die Probe ist mit einer Rauigkeit von RMS $<1.6 \mathrm{~nm}$ einigermaßen glatt. Daten von Oleg Shapoval.

Rauigkeit von $1.6 \mathrm{~nm}$ bestätigt.

Nach der ersten Charakterisierung wurde die Probe durch CAmillo Ballani nach dem in Kap. 3.4.2 beschriebenen Verfahren strukturiert, wozu Abb. 4.20 ein schematisches Bild zeigt. Dabei wurde nur die eine Hälfte der Probe strukturiert und die andere als Vollfilm belassen. Wie man sieht, sind dort in einem Raster mehrere Strukturen über die Probe verteilt, wobei die Breite des Steges aus Manganat zwischen $10 \mu \mathrm{m}, 30 \mu \mathrm{m}$, $50 \mu \mathrm{m}$ und $70 \mu \mathrm{m}$ variiert wurde. Über diese Stege liegen jeweils die Goldkontakte für eine Vierpunktmessung, die mittels Drahtbonden kontaktiert werden können. Ebenso sind je zwei kleine pfeilförmige Markierungen um jeden Steg gefasst, die das Auffinden erleichtern sollen.

\section{Widerstand}

Anschließend soll die Temperaturabhängigkeit des Widerstandes auch unter Einfluss eines magnetischen Feldes betrachtet werden. Dafür wurde der Film vor der Strukturierung und danach im PPMS mit einem Gleichstrom von maximal $50 \mu \mathrm{A}$ vermessen, wobei die beiden Strukturen mit den Koordinaten $(3,1)$ und $(4,4)$ (s. Abb. 4.20) mit Stegbreiten von $30 \mu \mathrm{m}$ bzw. $70 \mu \mathrm{m}$ untersucht wurden. Die Daten zeigt Abb. 4.21. Hierbei sieht man im unstrukturierten Film deutlich den Metall-Isolator-Übergang mit einer Übergangstemperatur von $T_{\mathrm{MI}} \sim 201 \mathrm{~K}$, was durch ein äußeres Magnetfeld unterdrückt und zu höheren Temperaturen verschoben wird. Jedoch bildet sich nach dem Strukturieren zusätzlich ein weiteres, schwächeres Maximum bei $T_{2 \text { nd }} \sim 182 \mathrm{~K}$ aus, welches bei beiden Strukturgrößen auftritt, jedoch für den breiteren Steg ausgeprägter ist. Dieses deutet darauf hin, dass der Effekt nicht, wie man sofort meinen könnte, mit der Größe der Struktur skaliert. Des Weiteren lässt sich auch dieses Nebenmaximum durch das Magnetfeld beeinflussen und ist schon bei $5 \mathrm{~T}$ kaum noch auszumachen. Zusätzlich fällt 


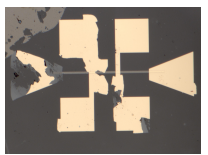

$(1,4) 10 \mu \mathrm{m}$

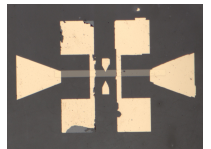

$(1,3) 30 \mu \mathrm{m}$

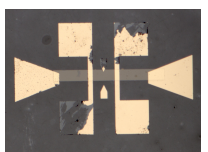

$(1,2) 50 \mu \mathrm{m}$

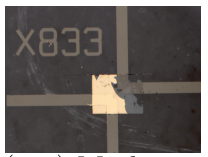

$(1,1)$ Marker

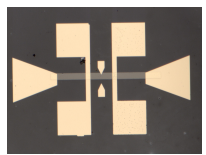

$(2,4) 30 \mu \mathrm{m}$

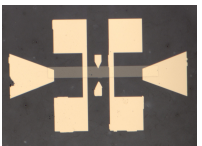

$(2,3) 50 \mu \mathrm{m}$

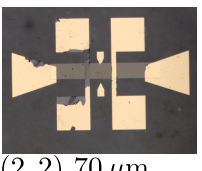

$(2,2) 70 \mu \mathrm{m}$

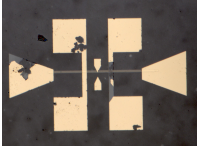

$(2,1) 10 \mu \mathrm{m}$

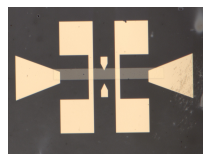

$(3,4) 50 \mu \mathrm{m}$

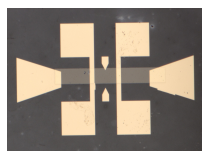

$(3,3) 70 \mu \mathrm{m}$

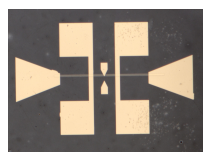

$(3,2) 10 \mu \mathrm{m}$

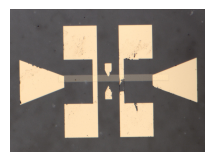

$(3,1) 30 \mu \mathrm{m}$

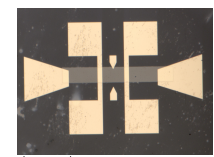

$(4,4) 70 \mu \mathrm{m}$

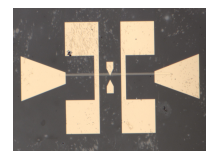

$(4,3) 10 \mu \mathrm{m}$

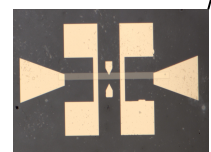

$(4,2) 30 \mu \mathrm{m}$

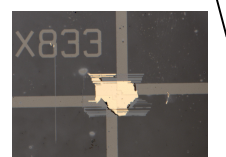

$(4,1)$ Marker

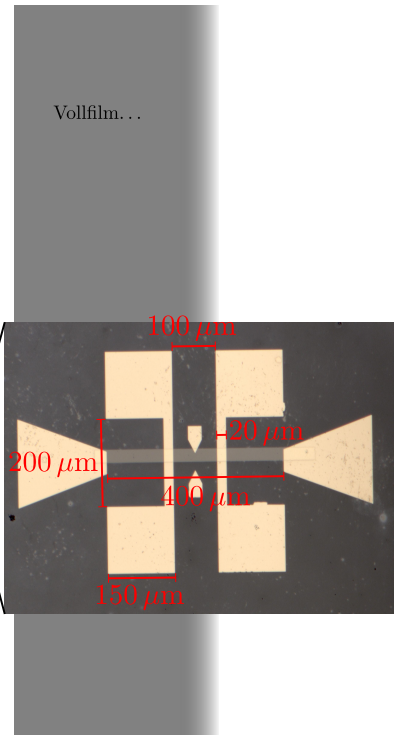

Abbildung 4.20.: Schematischer Aufbau der strukturierten LPCMO-Probe. Über vier Reihen und Spalten wurden Strukturen mit den Stegbreiten 10,30, 50,70 $\mu \mathrm{m}$ angeordnet. Das Manganat ist jeweils nur ein gerader Steg, über den die Goldkontakte gelegt und zwei pfeilartige Markierungen zum besseren Auffinden gelegt wurden. Die andere, hier nur angedeutete Probenhälfte verblieb unstrukturiert.

die Hysterese in den Strukturen auf, was gleichermaßen mit Anlegen eines Magnetfeldes quasi verschwindet.

Für das Auftreten eines solchen Verhaltens nach der Prozessierung kann es prinzipiell zwei generelle Möglichkeiten geben: Entweder wurde die Probe durch die Strukturierung ${ }^{9}$ chemisch oder physikalisch so modifiziert, dass dies als neue Eigenschaft sichtbar wird, oder das Merkmal war von vornherein in der Probe vorhanden, tritt jedoch erst durch die Strukturierung zutage. Gegen eine Veränderung spricht, dass die zweite Hälfte der Probe, die ja als Vollfilm verblieben ist, ebenso alle Prozessschritte wie z. B. das Ätzen durchlaufen hat, jedoch auch danach immer noch eine unveränderte Widerstandskurve zeigt. Weiterhin hätte bei derart großen Strukturen die Säure an den Rändern der Maske nicht genug Angriffsfläche um den Steg global zu verändern. Und auch die Chrom-GoldKontakte, deren Halt ja darauf beruht, dass das Chrom als Haftvermittler den Sauerstoff aus dem Manganat zieht, bedecken nur einen sehr geringen Teil und könnten lediglich die Oberfläche schädigen. Der Elektronenstrahl allerdings, der im ersten Schritt bei der Belichtung des Negativlacks die nachher gewünschte Struktur - also den Steg - schreibt, könnte eventuell Veränderungen hervorrufen, die sich aber aufgrund der sehr geringen Mobilität der Kationen auf die Sauerstoffionen beschränken würden. Jedoch sollte die meiste Energie im Fotolack deponiert werden, da dieser speziell empfindlich auf eine

\footnotetext{
${ }^{9}$ Für eine Beschreibung der Strukturierung siehe Kap. 3.4 .2 .
} 


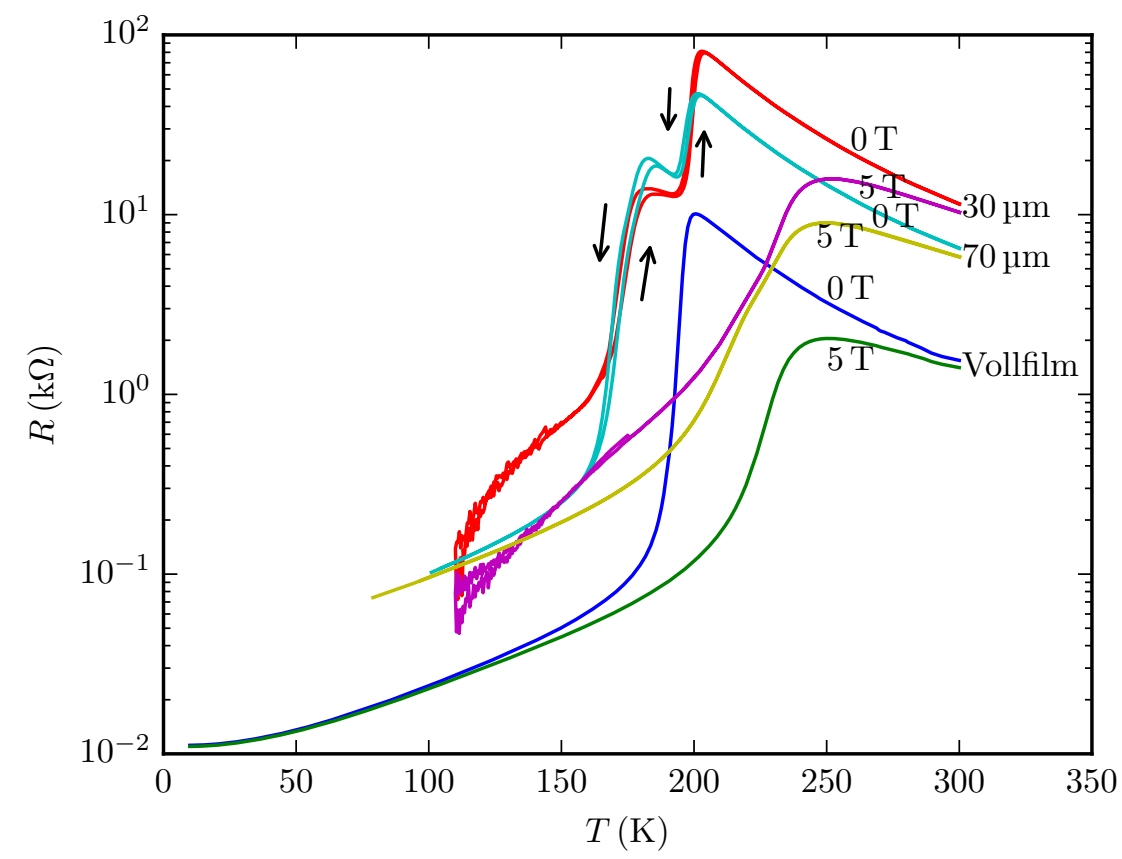

Abbildung 4.21.: Temperaturabhängigkeit des Gleichstromwiderstandes der LPCMO-Probe im PPMS gemessen, wobei als maximaler Strom $50 \mu \mathrm{A}$ verwendet wurde. Beim unstrukturierten Film wird das Maximum im Widerstand bei $T_{\mathrm{MI}} \sim 201 \mathrm{~K}$ durch das Magnetfeld unterdrückt und verschoben. Nach dem Strukturieren ist bei beiden Strukturgrüßen deutlich ein Nebenmaximum bei $T_{2 \text { nd }} \sim 182 \mathrm{~K}$ zu erkennen, welches ebenfalls durch das Magnetfeld unterdrückt wird. Weiter tritt bei beiden Maxima Hysterese auf, die unter Einfluss des Magnetfeldes verschwindet. Das Maximum im TCR des Vollfilms ist bei $T_{\mathrm{TCR}} \sim 196 \mathrm{~K}$. 
Belichtung mit dem Elektronenstrahl ist.

Nun findet man in der Literatur eine Veröffentlichung zu ganz ähnlichem Verhalten. So beobachteten auch Singh-Bhalla et al. SBH09 bei ihren Untersuchungen an Stegen, die mittels Focused Ion Beam (FIB) aus LPCMO-Filmen mit $x=0.33$ und $y=0.5$ hergestellt wurden, wobei die verwendeten Stegbreiten kleiner als $5 \mu \mathrm{m}$ waren. Dabei zeigten ihre Strukturen bis hinunter zu einer Größe von $2.5 \mu \mathrm{m}$ in der temperaturabhängigen Widerstandskurve die Eigenschaften des Vollfilms mit einer Übergangstemperatur bei $T_{\mathrm{MI}}=105 \mathrm{~K}$. Erst unterhalb dieser Breite traten stufenartige Nebenmaxima mit deutlicher Hysterese auf, die qualitativ dem hier ebenfalls gefundenen Verhalten entsprechen. Nun folgen sie als Erklärung der Argumentation von ZHAI et al. Zha+06, dass im phasenseparierten Zustand nahe des Übergangs die Ausdehnung der einzelnen Phasenbereiche gerade der Größenordnung des Steges entspricht und so beim weiteren Abkühlen kleinere noch isolierende Bereiche metallisch werden, wodurch sich der Perkolationspfad sprunghaft weiter fortsetzt, bis sich ein durchgängig metallischer Bereich ergibt.

Jedoch sind die hier untersuchten Strukturgrößen um den Faktor 10 bis 30 größer, sodass nicht die elektronische Phasenseparation [Ueh+99 für das Nebenmaximum verantwortlich sein kann. Vielmehr könnte eher eine zweite LPCMO-Phase mit leicht verschiedener Dotierung und damit anderer Übergangstemperatur vorhanden sein, deren charakteristische Größenordnung gerade einigen zehn Mikrometern entspricht. Dabei ergibt sich für das Auftreten allein bei Messungen an der strukturierten Probe dasselbe Argument wie im Falle der elektronischen Phasenseparation. Tatsächlich lässt sich der gemessene Widerstandsverlauf aus der Überlagerung zweier identischer Kurven des Vollfilms generieren, wobei für die zweite der Übergang zu tieferen Temperaturen hin verschoben und skaliert wurde, was Abb. 4.22 zeigt. So ergibt sich eine Verschiebung der zweiten Kurve um ungefähr $28 \mathrm{~K}$, was einer Dotierung von $y \sim 0.45$ entspräche, und ein Anteil einer Zweitphase für die $30 \mu \mathrm{m}$-Struktur von etwa $8 \%$. Allerdings war ja aus Abb. 4.21 bereits bekannt, dass für den Steg mit $70 \mu \mathrm{m}$ das Nebenmaximum ausgeprägter ist, sodass sich für den Fall dann auch ein Anteil von gut $21 \%$ ergibt.

Aufgrund des hier beobachteten Verhaltens ist eigentlich auszuschließen, dass es sich bei dem Nebenmaximum um eine Eigenschaft von zerstörtem Material handelt, denn es zeigt auch bekanntes CMR-Verhalten. Tatsächlich sind sogar die Nichtlinearitäten für das Nebenmaximum deutlich stärker, wie man später sehen wird.

\subsubsection{Positionierung}

Für die Positionierung des Anregungsflecks vom Laser auf der LPCMO Probe konnte die Thermospannung nicht verwendet werden, da kein unterscheidbares Signal auftrat, wenn die Struktur überquert wurde, auch nicht nahe der Temperatur des Metall-IsolatorÜbergangs. Daher wurde zum Ausrichten die Reflektivität (vgl. Kap. 3.10 betrachtet, die detaillierte Bilder der Oberfläche liefert und damit optimal für das Finden des Stegs geeignet ist. Abbildung 4.23 zeigt dafür beispielhaft eine Struktur, wobei die Kontaktpads aus Gold, auf denen man die Bonddrähte erkennen kann, und sogar der 


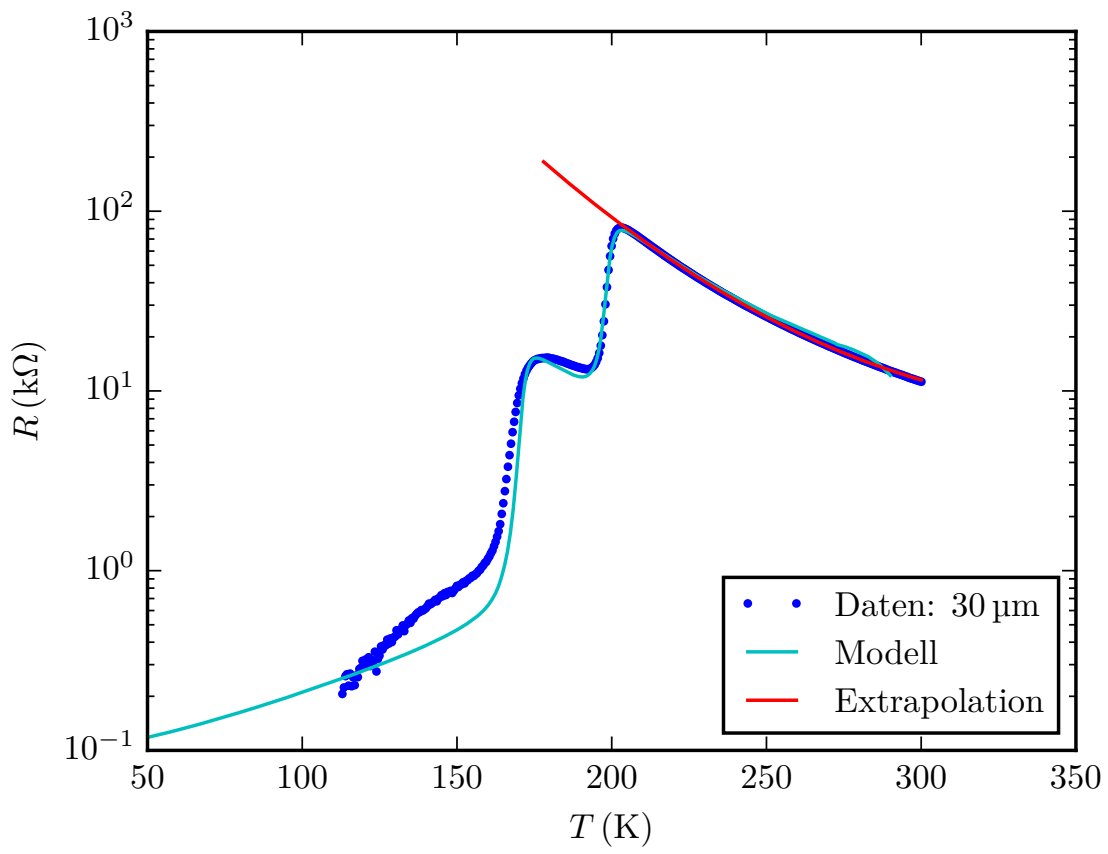

Abbildung 4.22.: Widerstandskurve der $30 \mu \mathrm{m}$-strukturierten LPCMO-Probe als Überlagerung zweier unterschiedlicher Dotierungen. Für das Modell wurde die Kurve des Vollfilms zweimal leicht versetzt übereinandergelegt, sodass die Überlagerung eine Koexistenz zweier unterschiedlich dotierter Phasen darstellen soll, deren Übergangstemperaturen sich um $\sim 28 \mathrm{~K}$ unterscheiden. Der Anteil der zweiten Phase, berechnet nach Extrapolation aus dem Verhältnis der Maixima, beträgt $\sim 8 \%$.

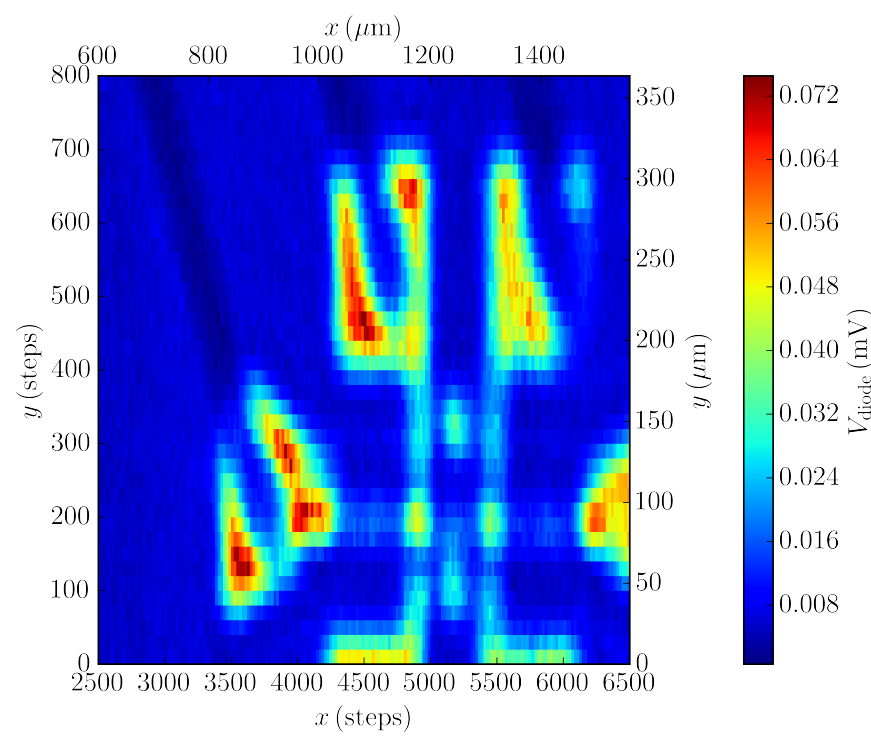

Abbildung 4.23.: Reflektivitätskarte der Probenoberfläche von LPCMO. Die Goldkontakte sind deutlich zu erkennen, wobei sie teils durch die Bonddrähte verdeckt werden. Der Steg aus dem Manganat ist leicht sichtbar und von den zwei pfeilartigen Markern umschlossen. 
Steg zu erkennen sind.

\subsubsection{Einfluss von Laseranregung auf die dritte harmonische Spannung}

Analog zum Messprotokoll von LBMO, welches in Kap. 4.1.5 beschrieben ist, wurden auch für LPCMO Messungen durchgeführt. Dieses konnte jedoch aufgrund der benötigten niedrigen Temperaturen nur am Aufbau in Göttingen geschehen und nicht auch zusätzlich in Greifswald. Nachdem also Zeitserien für die verschiedenen Temperaturen aufgenommen worden waren, welche im Anhang in Abb. A.4 gezeigt sind, konnte die Temperaturabhängigkeit für die erste und dritte harmonische Spannung und für den daraus berechneten dritten harmonischen Koeffizienten sowie die Auswirkung der Laseranregung ermittelt werden. Dieses ist in Abb. 4.24 gezeigt. Hier ist zu sehen, dass eine starke Erhöhung von $U_{3 \omega}$ und entsprechend auch von $K_{3 \omega}$, aber am Nebenmaximum des Widerstandes bei tieferen Temperaturen, vorliegt. Auch beim Hauptmaximum kann man eine leichtes Ansteigen des Signals feststellen, was jedoch in $U_{3 \omega}$ um einen Faktor 50 und in $K_{3 \omega}$ um einen Faktor 70 kleiner ist. Die mit dem Nebenmaximum verbundene Eigenschaft der Probe besitzt also offensichtlich deutlich stärkere nichtlineare Anteile als das Hauptmaximum. Für die Auswirkungen der Laseranregung ist festzustellen, dass sich der Widerstand, den ja $U_{\omega}$ widerspiegelt, nicht durch die Anregung verändert und die Kurven praktisch keine Differenzen aufweisen. Selbst die Temperaturverschiebung ist vernachlässigbar gering. In den Nichtlinearitäten - also $U_{3 \omega}$ und $K_{3 \omega}$ - ist jedoch eine deutliche Änderung im Signal am Nebenmaximum zu erkennen, sodass hier der Wert durch die Einwirkung des Lasers in beiden Größen um $2.4 \%$ abnimmt, was sich dann auch in der Differenz von $K_{3 \omega}$ in Abb. 4.25 zeigt. Für das Hauptmaximum hingegen ist keine Veränderung zu erkennen. Weiterhin zeichnet sich in $K_{3 \omega}$ auf dem Maximum eine Unterstruktur ab und man sieht ein Doppelmaximum, welches jedoch in den anderen beiden Kurven nicht zu sehen ist. Aber schon in $U_{3 \omega}$ hat das Maximum eine seltsam asymmetrische Form, sodass es den Anschein hat, als sei es auf der linken Seite abgeschnitten. Das könnte bedeuten, dass $U_{3 \omega}$ noch weiter ansteigen würde, aber vielleicht kommen durch den hohen Wert kapazitive Beiträge der Probe in der Wechselfeldmessung zum Tragen, die das Signal abschwächen. Auch wiederholte Messungen an dieser Probe zeigten ein asymmetrisches Maximum, was für die Erklärung spricht.

\section{Magnetfeldabhängigkeit}

Für LPCMO wurde auch die Magnetfeldabhängigkeit bis $5 \mathrm{~T}$ betrachtet, indem wieder die im Anhang in Abb. A.5 gezeigten Zeitserien aufgenommen und ausgewertet wurden. Abbildung 4.26 stellt die Daten dar. Dabei sieht man, dass sich für das Nullfeld durch den Laser die schon bekannte Abschwächung des Signals um 2\% ergibt und für größer werdende Felder sowohl die Werte als auch die Unterschiede zwischen Anregung und keiner Anregung abnehmen. 

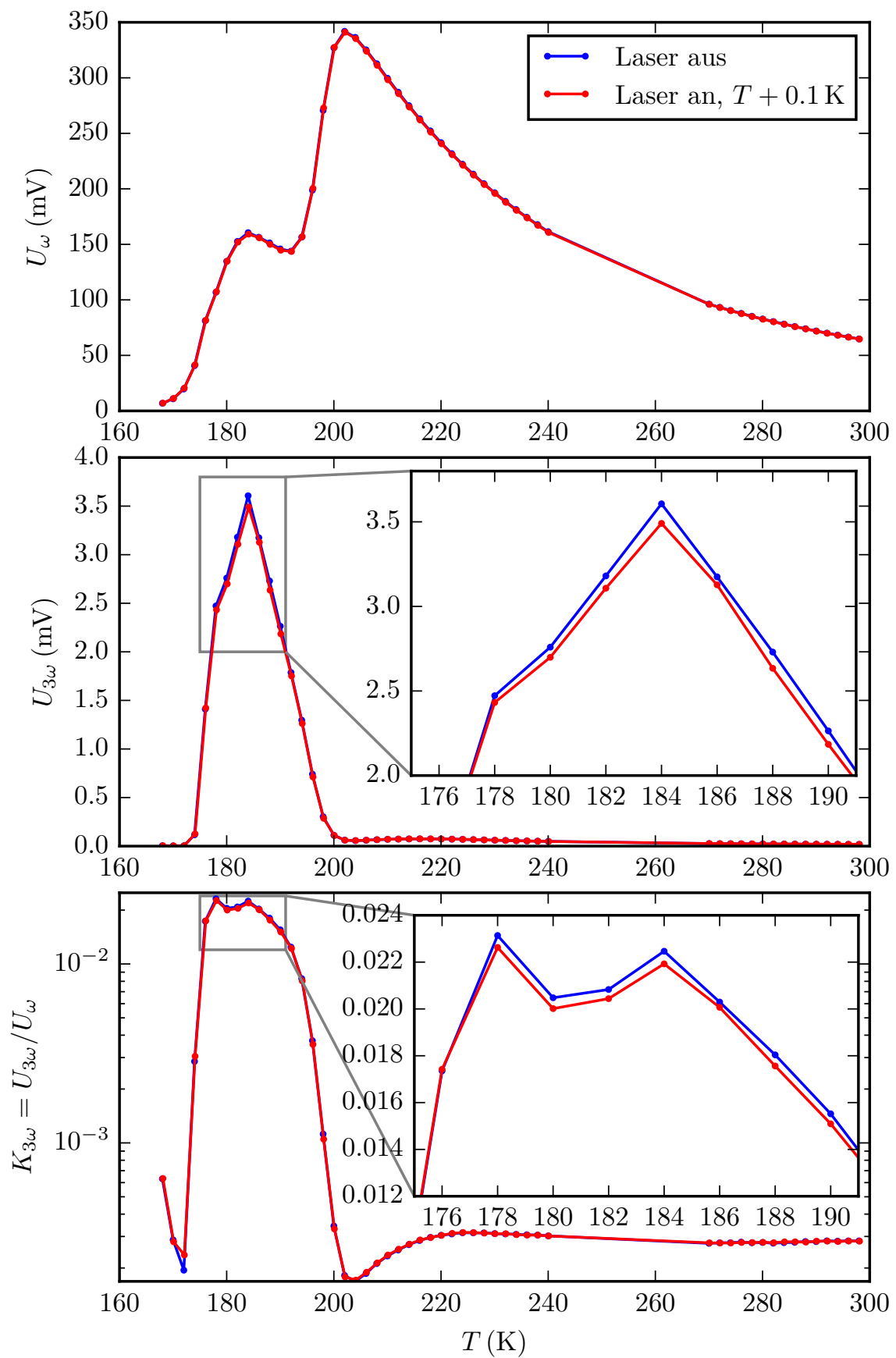

Abbildung 4.24.: Temperaturabhängigkeit der ersten und dritten harmonischen Spannung und des dritten harmonischen Koeffizienten von LPCMO unter Laseranregung. 


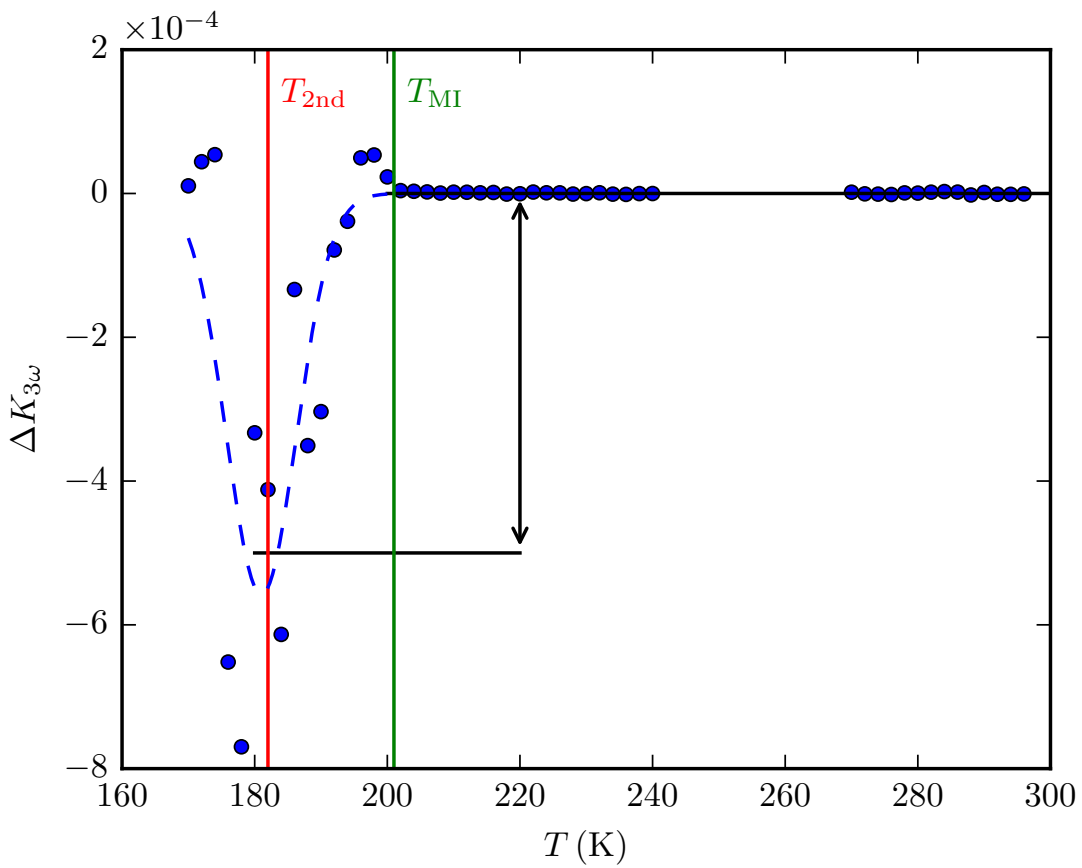

Abbildung 4.25.: Änderung des dritten harmonischen Koeffizienten durch Laseranregung bei LPCMO, bestimmt aus dem unteren Graphen in Abb. 4.24 In der Nähe der Temperatur des zweiten Widerstandsmaximums $T_{2 \text { nd }}$ stellt man eine Erniedrigung von $2.4 \%$ fest. 


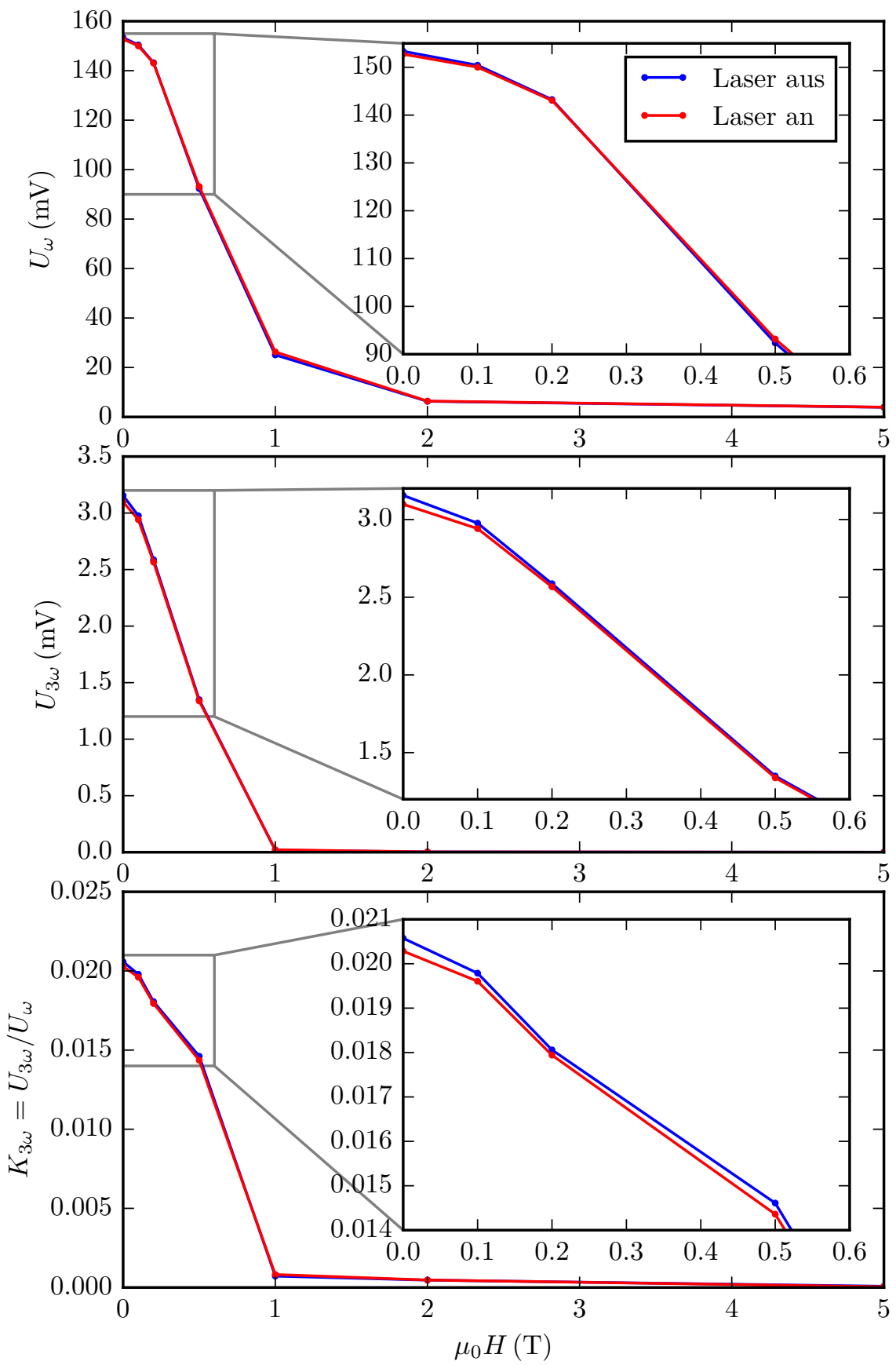

Abbildung 4.26.: Magnetfeldabhängigkeit der ersten (oben) und dritten harmonischen Spannung (mittig) und des dritten harmonischen Koeffizienten (unten) von LPCMO bei $184 \mathrm{~K}$. Unter Laseranregung im Nullfeld ergibt sich eine Änderung von $K_{3 \omega}$ von $\sim-2 \%$, die mit zunehmendem Magnetfeld verschwindet. 


\section{Diskussion}

Dieses Kapitel soll die gewonnen Messdaten interpretieren und mit Modellen oder Phänomenen erklären oder mögliche Ansätze dafür liefern, welche dabei in einen größeren Zusammenhang des Themas eingeordnet werden.

\subsection{Kontakte}

Zu Beginn sollen kurz mögliche Probleme, die durch die Kontakte entstehen könnten, diskutiert und ausgeschlossen werden. Denn bei der Strukturierung wird als Haftvermittler für die Goldkontakte eine ungefähr $10 \mathrm{~nm}$ dünne Schicht aus Chrom aufgedampft, welche auf dem Manganat gut haftet, da sie aus den obersten Lagen den Sauerstoff herauszieht und damit eine dünne Schicht Chromoxid bildet (Abb. 5.1). Nun kann aber der dadurch entstehende sauerstoffdefizitäre Bereich im Manganat nur sehr klein sein, da bei Raumtemperatur die Mobilität des Sauerstoffs nicht ausreicht, um tiefer in die Chromschicht einzudringen.

Eine weitere Schwierigkeit könnten diese unterschiedlichen Materialien und zugehörigen Grenzschichten bei einer elektrischen Messung verursachen, aber da es sich um eine Vierpunktmessung handelt, sollten jegliche Kontaktwiderstände keine Rolle spielen.

Weiterhin sind auch die Kontaktwiderstände in den relevanten Temperaturbereichen kleiner als der Probenwiderstand und erst bei tiefen Temperaturen erreichen sie dieselbe Größenordnung (vgl. Abb. A.8 im Anhang).

\subsection{Strom-Spannungs-Kennlinie}

Von LBMO wurden in Kap. 4.1.8 Strom-Spannungs-Kennlinien für verschiedene Temperaturen gezeigt, welche deutlich nichtlineares Verhalten besitzen und Asymmetrie aufweisen, wenn der Strom durch den strukturierten Steg fließen muss. Daraus kann man für die gemessenen Temperaturen den Widerstand in Abhängigkeit der Spannung oder des Stroms gewinnen, indem man für je eine feste Temperatur $T$ die Werte $U, I$ und $R=U / I$ bestimmt, deren Kurven Abb. A.6 im Anhang zeigt. Durch Interpolation dieser Kurven kann man für ein fest gewähltes $U$ oder $I$ auch den spannungs- oder stromabhängigen Temperaturverlauf des Widerstandes bestimmen (Abb. 5.2). Wie dort zu erkennen ist, wird das Maximum des Übergangs durch höhere Spannung bzw. höheren Strom unterdrückt, aber nur für große Spannungen und Ströme leicht zu höheren Temperaturen verschoben. Letzteres würde man zumindest nicht durch joulesche Erwärmung erwarten, was damit als alleinige Erklärung ausscheidet. Dennoch soll überprüft werden, 


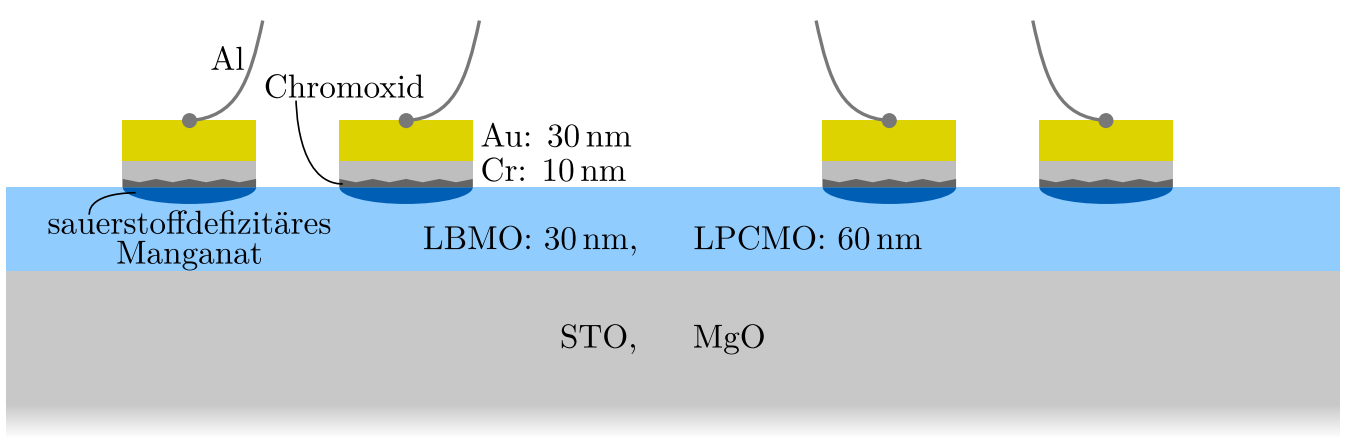

Abbildung 5.1.: Schema der Kontaktbeschaffenheit auf den Proben. Das Chrom als Haftvermittler zieht den Sauerstoff aus den obersten Lagen des Manganats und schädigt es damit, darunter ist die Schicht jedoch intakt. Trotz der verschiedenen Materialien in den Kontakten spielen aufgrund der Vierpunktgeometrie die entstehenden Kontaktwiderstände keine Rolle.
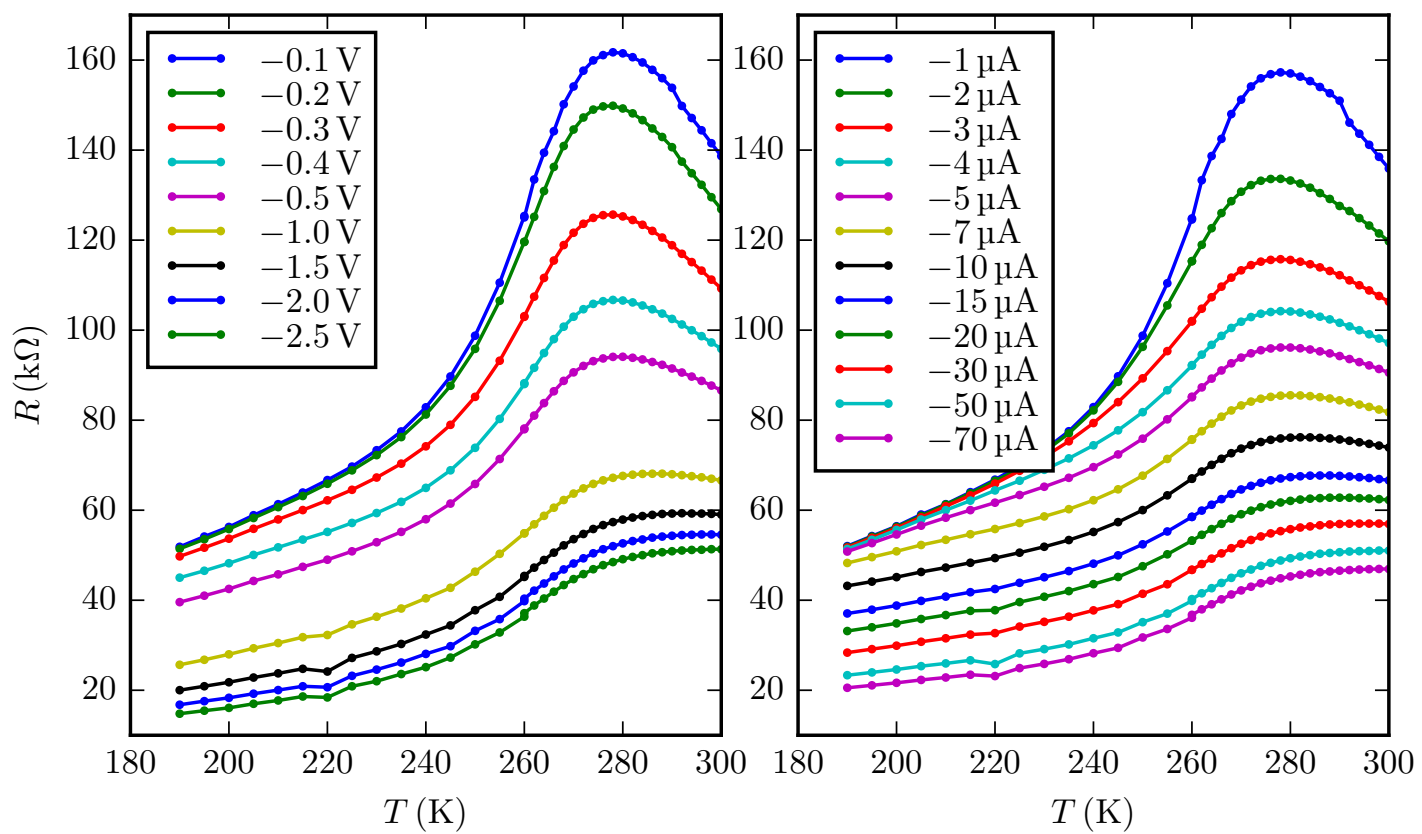

Abbildung 5.2.: Temperaturverlauf des Widerstandes für verschiedene Spannungen bzw. Ströme, gewonnen aus den temperaturabhängigen Strom-Spannungskennlinien aus Abb.4.17 Mit zunehmender Spannung bzw. zunehmendem Strom wird das Maximum im Widerstand unterdrückt, seine Lage ändert sich jedoch nur leicht bei großen Spannungen und Strömen. Für sehr kleine Spannungen und Ströme laufen die Kurven bei tiefen Temperaturen wieder zusammen, für höhere ist der Widerstand insgesamt abgesenkt. 


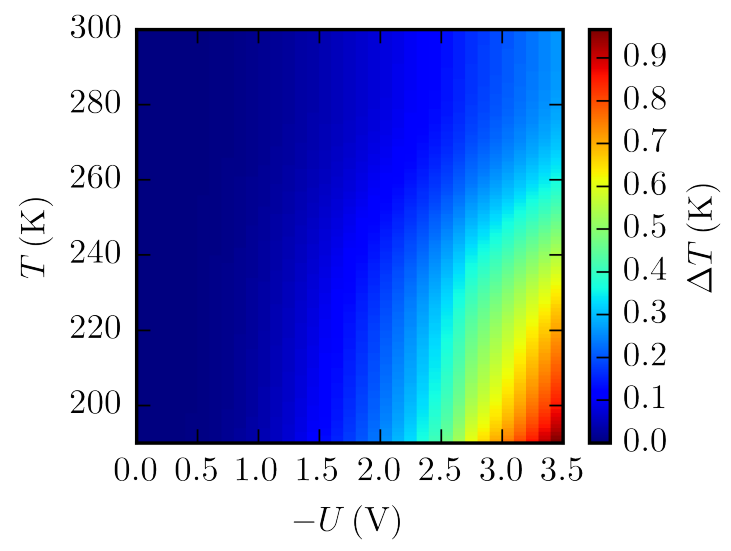

Abbildung 5.3.: Joulesche Erwärmung iterativ nach Gl. 5.1 mithilfe des Widerstandes und $\left\langle\kappa_{\mathrm{s}}\right\rangle \sim 15 \mathrm{~W} / \mathrm{m} \cdot \mathrm{K}$ und $l=120 \mu \mathrm{m}$ berechnet.

wie stark der Einfluss dieses Effektes ist. So kann man für die Temperaturänderung eines Stegs $\Delta T \sim 2 P / l \kappa_{\mathrm{s}}$ annehmen, wenn $P / l$ die eingetragene Leistung pro Länge ist und man davon ausgeht, dass der Abtransport der Wärme mit der thermischen Leitfähigkeit $\kappa_{\mathrm{S}}$ über das Substrat erfolgt, welches an das thermische Bad mit der Basistemperatur gekoppelt ist [LTA03; $\mathrm{Pad}+04$. Daraus erhält man

$$
\Delta T \approx \frac{2 U^{2}}{R(T+\Delta T) \cdot l \kappa_{\mathrm{s}}},
$$

wobei dieses eine implizite Gleichung ${ }^{1}$ und zusätzlich $\kappa_{\mathrm{s}}$ eigentlich auch temperaturabhängig ist, aber für Letzteres ein Mittel von $\left\langle\kappa_{\mathrm{s}}\right\rangle \sim 15 \mathrm{~W} / \mathrm{m} \cdot \mathrm{K}[\mathrm{Oh}+11$ angenommen wird. Wertet man diese Beziehung unter Nutzung der aus den Strom-Spannungs-Kennlinien bestimmten Widerstandsdaten iterativ aus, so erhält man Abb. 5.3. aus der man eine maximale Erwärmung von $\Delta T<1 \mathrm{~K}$ entnehmen kann. Selbst bei sehr konservativer Abschätzung mit einer maximalen Spannung am Steg von $3.5 \mathrm{~V}$ und einem kleinsten Widerstand von $\sim 10 \mathrm{k} \Omega$ ergibt sich maximal eine mögliche Temperaturänderung von $\Delta T<1.4 \mathrm{~K}$, wobei hier sogar die Zunahme des Widerstandes aufgrund der Erwärmung ignoriert wurde. Auch die Simulationen mittels finite Elemente an PCMO-Sandwich-Strukturen von SCHERFF Sch15] kamen zu dem Ergebnis, dass keine signifikante Erwärmung für Stromdichten von $<10^{9} \mathrm{~A} / \mathrm{m}^{2} \mathrm{zu}$ erwarten ist, was hier mit $j<2.3 \cdot 10^{8} \mathrm{~A} / \mathrm{m}^{2}$ deutlich unterschritten wird. Damit sollte joulesches Aufheizen am Steg kaum Auswirkungen auf die Strom-Spannungs-Kennlinie haben.

Folglich muss eine andere Ursache für die starke Änderung des Widerstandes bei größerer Spannung oder größerem Strom vorhanden sein. In seinen Untersuchungen an PCMO-Sandwich-Strukturen fand SCHERFF [Sch15] eine temperaturunabhängige Spannungsschwelle, ab der nicht-ohmsches Verhalten auftrat, jedoch für die Stromstärke keine entsprechende Schwelle. Auch für die hier untersuchte Probe lässt sich in Abb. 5.4

\footnotetext{
${ }^{1}$ Diese Rückkopplung sorgt dafür, dass nur oberhalb des Übergangs, wo $\mathrm{d} R / \mathrm{d} T<0$, aber $R$ sowieso schon deutlich größer ist, eine Verstärkung der Erwärmung stattfinden könnte, jedoch unterhalb mit $\mathrm{d} R / \mathrm{d} T>0$ eine Abschwächung des Effekts erfolgt.
} 

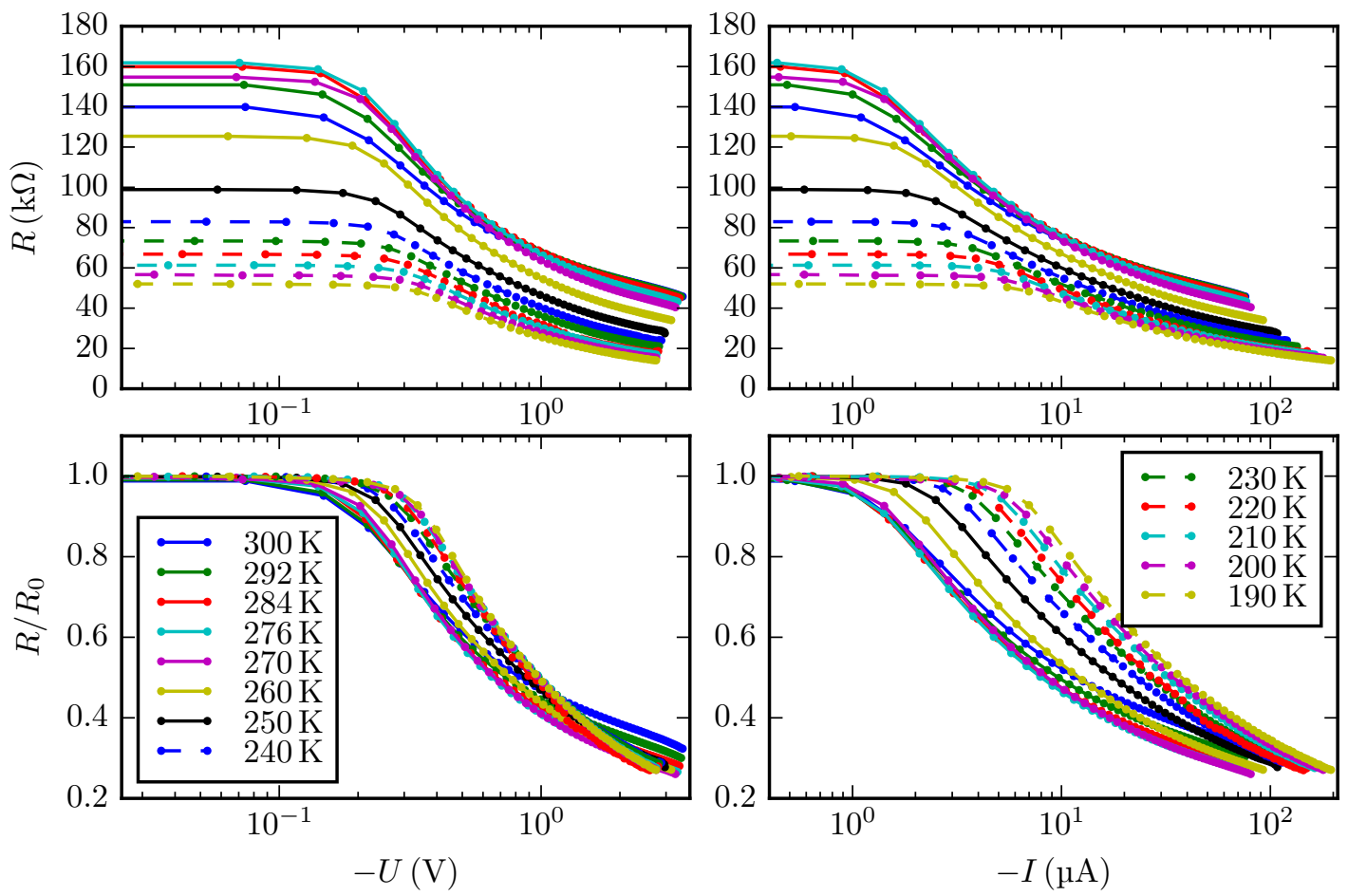

Abbildung 5.4.: Widerstand in Abhängigkeit der Spannung und des Stroms von LBMO für ausgewählte Temperaturen, gewonnen aus den Strom-Spannungs-Kennlinien. Ab einer Spannung von $0.1 \ldots 0.25 \mathrm{~V}$ liegt kein ohmsches Verhalten mehr vor, für den Strom lässt sich keine gemeinsame Quelle ausmachen. Die gegenüber den anderen Temperaturen erhöhten Werte der Kurven für $T>T_{\mathrm{MI}}$ bei hohen Spannungen und Strömen ergeben sich aus der leichten Verschiebung des Maximums in Abb. 5.2 zu höheren Temperaturen. Vollständige Kurven und positiver Teil im Anhang in Abb. A.6 bzw. Abb. A.7. 
erkennen 22, dass unabhängig von der Temperatur ab einem kleinen Spannungsbereich der Widerstand sinkt, während für den Strom kein solcher Bereich ausgemacht werden kann. Somit liegt die Vermutung nahe, dass hier ein spannungsaktivierter Prozess vorliegt, der bei $0.1 \ldots 0.25 \mathrm{~V}$ einsetzt, was für den Steg mit einer Länge von $120 \mu \mathrm{m}$ Feldstärken von $0.8 \ldots 2.1 \mathrm{kV} / \mathrm{m}$ entspricht. Dieses ist knapp zwei Größenordnungen geringer als die Feldstärken von $100 \mathrm{kV} / \mathrm{m}$, die WesthäUser et al. Wes+06 in PCMO für einen elektrisch induzierten Isolator-Metall-Übergang finden. Tatsächlich zeigen auch theoretische Rechnungen [GWT03, dass mikroskopisch Felder von $10^{7} \mathrm{~V} / \mathrm{m}$ nötig sind, um im phasenseparierten Zustand die isolierenden Bereiche zu verkleinern. Jedoch können gerade aufgrund der Phasenseparation lokal sehr hohe Feldstärken entstehen und in einer sehr vereinfachten Annahme würden isolierende Bereiche mit einer Ausdehnung von $10 \mathrm{~nm}$ die erforderliche Feldstärke hervorrufen 3 Weiterhin ist auch die leichte Verschiebung des Maximums zu höheren Temperaturen in Abb. 5.2 ein Indiz für den Einfluss des elektrischen Feldes. Damit spricht vieles dafür, dass durch die angelegte Spannung oder evtl. auch durch den fließenden Strom die Probe in den metallischen Zustand getrieben wird, womit man auch hier von einem elektrisch induzierten IsolatorMetall-Übergang sprechen kann. Daraus lässt sich dann die stark nichtlineare Form der Strom-Spannungs-Kennlinien erklären. Es sei jedoch noch einmal angemerkt, dass in den Wechselfeld-Messungen der ersten und dritten harmonischen Spannung die hier diskutierten Nichtlinearitäten und ebenfalls die Asymmetrie keine Rolle spielen, da bei den dort verwendeten kleinen Spannungen bzw. Strömen die Probe noch ohmsches Verhalten zeigt.

\subsection{Wärmedissipation}

Mit der Messung, deren Ergebnisse in Kap. 4.1.7 beschrieben sind, sollte untersucht werden, wie schnell durch den Laser in die Probe eingebrachte Wärme an das Substrat und damit an das thermische Bad abgegeben werden kann. An die dort gezeigten Daten können jetzt Exponentialfunktionen angepasst werden, um daraus eine charakteristische Frequenz bzw. Zeitskala zu bestimmen, was in Abb. 5.5 dargestellt ist. Dort sind eindeutig zwei Bereiche unterschiedlicher Relaxationszeit zu erkennen, die bei $\sim 273 \mathrm{~K}$ getrennt sind, wobei für große Temperaturen eine schnelle Dissipation mit $\tau_{\mathrm{h}} \sim 2 \mathrm{~ms}$ und für tiefe Temperaturen eine langsame mit $\tau_{\mathrm{t}} \sim 20 \mathrm{~ms}$ stattfindet.

Nun liegt diese Temperatur verdächtig nahe an der Schmelztemperatur von Wasser, sodass hier ein Zusammenhang vermutet werden könnte. Und obwohl die Messungen

\footnotetext{
${ }^{2}$ Hier sind nur die Daten der negativen Spannungen und Ströme dargestellt. Die vollständigen Kurven und der positive Ast ist im Anhang in Abb. A.6 bzw. Abb. A.7, wobei letzterer qualitativ ähnliches Verhalten zeigt

${ }^{3}$ Eigentlich müsste man wie Gu et al. GWT03 ein mindestens zweidimensionales Widerstandsnetzwerk aus metallischen und isolierenden Bereichen betrachten, um eine genauere Aussage über Spannung und Ausdehnung zu machen. Aber eben diese Rechnungen gelangen auch zu nanometergroßer Ausdehnung bei einer von außen angelegten Spannung von $0.01 \mathrm{~V}$, was zu den hier bestimmten Werten passt.
} 

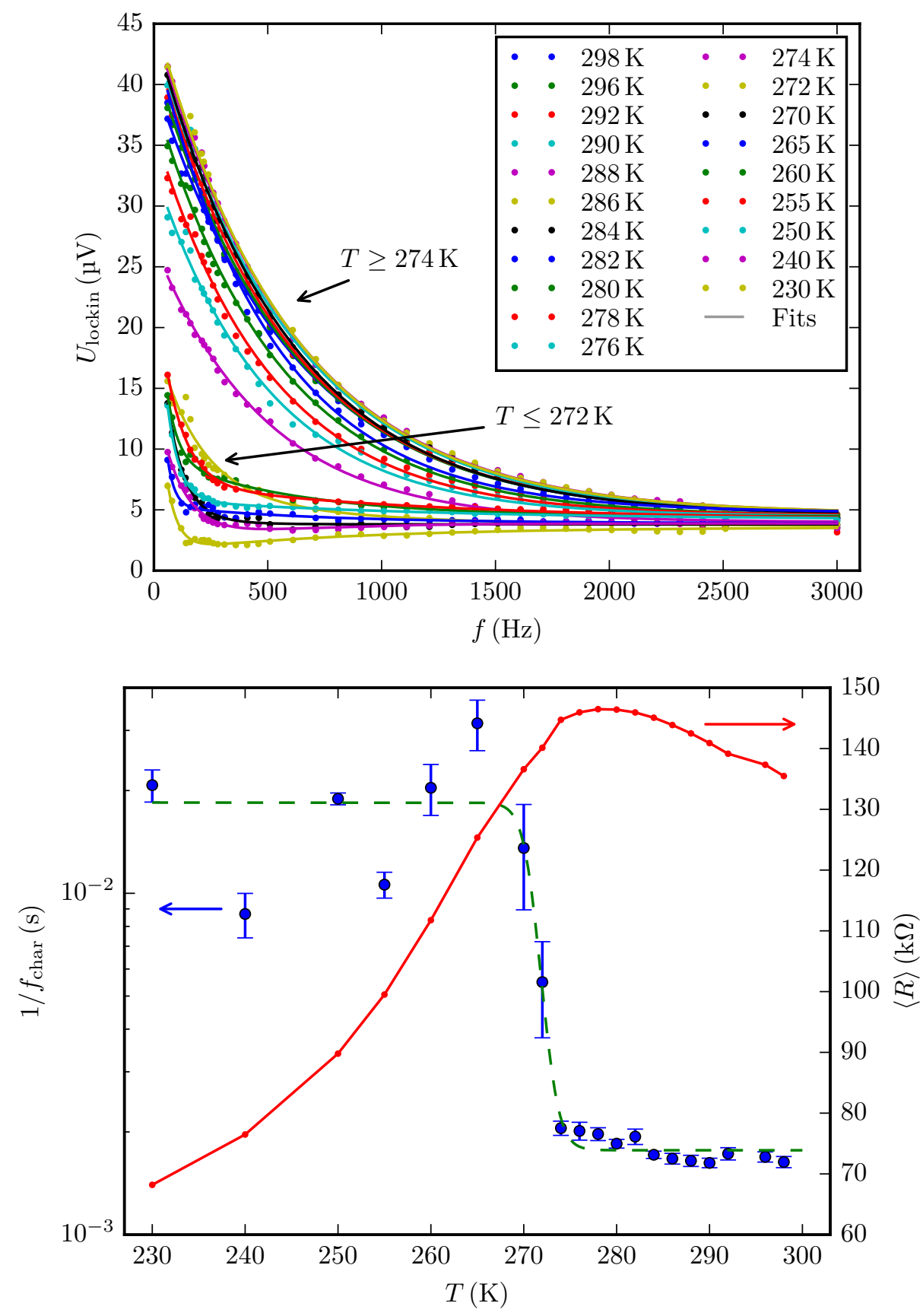

Abbildung 5.5.: Fit von Exponentialfunktionen an die Änderung des Gleichstromwiderstandes bei periodischer Laseranregung (oben) und Temperaturabhängigkeit der charakteristischen Zeitskala (unten). Ebenso wurde aus den zusätzlich aufgezeichneten Messdaten zu jeder Frequenzserie ein mittlerer Widerstand bestimmt. Zwei unterschiedliche Zeitskalen für hohe und für tiefe Temperaturen sind zu sehen, deren Trennung bei der Übergangstemperatur liegt. Ihre Größenordnungen sind $\tau_{\mathrm{h}} \sim 2 \mathrm{~ms}$ und $\tau_{\mathrm{t}} \sim 20 \mathrm{~ms}$. 
bei leichtem Vakuum im PPMS mit einem Druck von ungefähr 10 mbar durchgeführt wurden, liegt dennoch die Schmelztemperatur von Wasser unter diesen Bedingungen bei $0^{\circ} \mathrm{C}$, da die Phasengrenze zwischen Wasser und Eis bis zum Tripelpunkt bei 6.12 mbar GJJ76 im $p$-T-Diagramm nahezu senkrecht verläuft. Jedoch wird die Probenkammer nach dem Einbau der Probe mehrfach mit Helium gespült, sodass beim Evakuieren aufgrund der gesenkten Siedetemperatur des Wassers unter die Raumtemperatur der größte Teil entfernt ist. Des Weiteren könnte eine mögliche anhaltende Beeinflussung nur durch eine Eisschicht auf optischen Komponenten oder der Probe entstehen und dafür ist der Absorptionskoeffizient von Eis bei $800 \mathrm{~nm}$ mit $\alpha \sim 21 / \mathrm{m}$ WB08 viel zu klein, denn selbst bei einer Eisdicke von $1 \mathrm{~mm}$ wäre die Intensität des Lasers lediglich um $0.2 \%$ geschwächt. Weiterhin kann eigentlich jegliche durch Eis verursachte Abschwächung des Lasers ausgeschlossen werden, da dieses nur das Messsignal insgesamt schwächen würde, aber keine Veränderung der charakteristischen Zeitskala verursachen könnte (siehe Abb. A.9 im Anhang). Und zuletzt war in keiner anderen Messung mit oder ohne Einwirkung des Lasers ein Einfluss von Wasser sichtbar. Damit kommt die Entstehung von Eis als Ursache nicht infrage und es bleibt allein eine Eigenschaft der Probe als Erklärung übrig.

So ändern sich in der Nähe der hier trennenden Temperatur aufgrund des Übergangs die physikalischen Eigenschaften wie elektrischer Transport, magnetische Ordnung und Reichweite von Korrelationen, wofür als Beispiel die Widerstandskurve in Abb. 5.5 betrachtet werden kann. Allerdings sollte eine Zunahme der elektrischen Leitfähigkeit mit steigender Wärmeleitfähigkeit einhergehen, was bei tieferen Temperaturen damit zu einer kürzeren Zeitskala führen müsste. Da jedoch des Gegenteil beobachtet wird, muss es sich eher um charakteristische Zeiten der Wärmedissipation vom Elektronensystem an das Gitter handeln. Hier wird bei $T<272 \mathrm{~K}$ ein Kanal geschlossen, der die Relaxation um eine Größenordnung von $\tau_{\mathrm{h}} \sim 2 \mathrm{~ms}$ auf $\tau_{\mathrm{t}} \sim 20 \mathrm{~ms}$ erhöht. Dafür könnte die einsetzende magnetische Ordnung eine Ursache sein, was auch MoshnYAGA et al. Mos+04 bei ihren Untersuchungen der Photoleitfähigkeit von LCMO beobachtet haben. Denn dort verlangsamte sich beim Erreichen der ferromagnetischen Phase die Relaxation der durch Laseranregung hervorgerufenen Änderung von $1.6 \mathrm{~s}$ auf $22 \mathrm{~s}$, was auch einer Größenordnung entspricht. Leider wird dabei keine explizite Erklärung für die langsamere Zeitskala genannt, jedoch der nach schneller photoinduzierter Demagnetisierung auftretende Remagnetisierungsprozess angesprochen, der dafür verantwortlich sein kann und von dem auch bekannt ist [Mül+09], dass er in der ferromagnetischen Phase der Manganate aufgrund von unterdrückter Wechselwirkung zwischen den Ladungsund Spin-Freiheitsgraden der Elektronen zwei Größenordnungen langsamer ist als z. B. bei Nickel. Dieser Umstand könnte auch im hier beschriebenen Fall die Ursache sein, sodass in der isolierenden, paramagnetischen Hochtemperaturphase die durch den Laser deponierte Energie schnell aus dem Elektronensystem dissipieren kann, während bei der ferromagnetischen Ordnung für tiefe Temperaturen die Dissipation jedoch über

\footnotetext{
${ }^{4}$ Der Absorptionskoeffizient wurde mithilfe von Gl. 2.15 aus dem Imaginärteil des Brechungsindexes $\kappa \approx 1.28 \cdot 10^{-7}$ berechnet.
} 


\begin{tabular}{lrr}
\hline & Göttingen & Greifswald \\
\hline System & Femtolasers Femtosource fusion 20 & Coherent RegA 9040 \\
Pulsdauer & $120 \mathrm{fs}$ & $45 \mathrm{fs}$ \\
Repetitionsrate & $74.9 \mathrm{MHz}$ & $250 \mathrm{kHz}$ \\
Leistung & $(\max ) .10 \mathrm{~mW}$ & $100 \mathrm{~mW}$ (oder mehr) \\
Pulsenergie & $0.13 \mathrm{~nJ}$ & $400 \mathrm{~nJ}$ \\
Strahltaille (FWHM) & $40 \mu \mathrm{m}$ & $80 \mu \mathrm{m}$ \\
Peakleistung & $5 \cdot 10^{7} \mathrm{~W} / \mathrm{cm}^{2}$ & $10^{11} \mathrm{~W} / \mathrm{cm}^{2}$ \\
Fluenz & $0.011 \mathrm{~mJ} / \mathrm{cm}^{2}$ & $7.96 \mathrm{~mJ} / \mathrm{cm}^{2}$ \\
Photonendichte & $0.16 \cdot 10^{-3} 1 /$ u.c. & $0.121 /$ u.c. \\
\hline
\end{tabular}

Tabelle 5.1.: Vergleich der Leistungsdaten der Lasersysteme. Peakleistung, Fluenz und Photonendichte beziehen sich dabei jeweils auf einen Puls.

die Magnetisierungsänderung sehr langsam stattfindet. Tatsächlich fanden auch PFAHL et al. $\mathrm{Pfa}+17$ in ihren Reibungsexperimenten mithilfe von Rasterkraftmikroskopie (engl. Atomic Force Microscopy (AFM)) bei LSMO eine Änderung der Reibungskräfte in Abhängigkeit der Temperatur, die sie ebenfalls auf den Wechsel der Relaxationszeiten am Übergang aufgrund der Schließung des Dissipationskanals zurückführen.

\subsection{Einfluss von Laseranregung auf die dritte harmonische Spannung}

Die Untersuchungen zur dritten harmonischen Spannung an den beiden verschiedenen Manganatsystemen LBMO und LPCMO sind in Kap. 4.1.5 beschrieben. Dabei gibt der dritte harmonische Koeffizient $K_{3 \omega}$, der sich aus dem Verhältnis aus dritter und erster harmonischer Spannung berechnet, die Konzentration der korrelierten Polaronen an, welche am Metall-Isolator-Übergang ihr Maximum erreichen sollte. Indirekt wird dadurch auch qualitativ die Elektron-Phonon-Wechselwirkung angezeigt und für LBMO findet man eine Konzentration von $0.22 \ldots 0.35 \%$ und für LPCMO $2 \ldots 2.5 \%$ bei $T_{2 \text { nd }}$ und $\sim 0.32 \%$ bei $T_{\mathrm{MI}}$. Ebenso wird in der Literatur bei optimaler Dotierung über Konzentrationen von weniger als $0.5 \%$ berichtet Mos+14, MS11] und bei Dotierungen von $x \sim 0.5$ ein Bereich $2 \ldots 4 \%[\mathrm{Dao}+02, \operatorname{Rad}+97]$. Letzteres spricht erneut dafür, dass die Eigenschaften beim zweiten Widerstandsmaximum der LPCMO-Probe von einer Phase mit anderer Dotierung herrühren, wobei in dieser die Korrelationen ausgeprägter sind.

Um nun die Einwirkung von Laserlicht auf die dritte harmonische Spannung bzw. den dritten harmonischen Koeffizienten zu beurteilen, muss man berücksichtigen, dass die Untersuchungen an zwei unterschiedlichen Messaufbauten durchgeführt wurden. Daraus ergeben sich aufgrund der unterschiedlichen verwendeten Laser verschiedene Leistungsparameter, die in Tab. 5.1 aufgeführt sind. Dabei stellen sich große Unterschiede 
nicht nur in der Leistung, sondern aufgrund der stark abweichenden Repetitionsraten besonders in der Fluenz und der Photonendicht 5 dar und somit ist als Antwort der Systeme durchaus Unterschiedliches zu erwarten. Daher zeigen die Messungen auch, dass die Systeme qualitativ und quantitativ verschiedenen auf die Laseranregung reagieren.

So findet man bei LBMO für die Messungen in Göttingen mit kleiner Leistung eine leichte Erhöhung des dritten harmonischen Koeffizienten $K_{3 \omega}$ um etwa $1 \%$, was damit auch eine entsprechende Zunahme der Dichte der korrelierten Polaronen bedeutet. Allerdings zeigt dieselbe Probe bei Anregung mit der zehnfachen Laserleistung in Greifswald und der damit verbundenen 700-fachen Fluenz und Photonendichte eine Abnahme des Koeffizienten und der Polaronendichte um ungefähr 2.5\%. Ebenso ergibt sich bei der LPCMO-Probe eine negative Änderung von 2.4\%, wobei dieses nur mit der geringen Leistung in Göttingen gemessen wurde, da der Übergang von LPCMO tiefe Temperaturen erfordert, die lediglich mithilfe des PPMS erreicht werden können. Dabei waren alle Ergebnisse bei verschiedenen Messdurchläufen konsistent reproduzierbar, auch wenn die einzelnen Durchführungen zum Teil längere Zeit auseinander lagen.

$\mathrm{Zu}$ berücksichtigen ist noch, dass die Stege aufgrund ihrer länglichen Ausdehnung von $120 \mu \mathrm{m}$ (LBMO) bzw. $100 \mu \mathrm{m}$ (LPCMO) geometrisch nur teilweise durch den Anregungsfleck der Laser (s. Tab. 5.1) ausgeleuchtet wurden und damit auch eine Anregung nur in diesem Bereich vorlag. Nun sind die Bereiche mit und ohne Anregung auf den Stegen in Reihe angeordnet und so kann der Effekt bezogen auf die entsprechenden Flächen skaliert werden, um nur die Auswirkung auf den angeregten Teil zu erhalten. Bei den Messungen von LBMO in Göttingen entsprach das Flächenverhältnis einem Drittel, was einen korrigierten Effekt in $K_{3 \omega}$ von $+3 \%$ bedeutet, und in Greifswald war der angeregte Anteil zwei Drittel, woraus sich eine Änderung von $-3.75 \%$ ergibt. Von dem LPCMO-Steg wurde mit dem Aufbau in Göttingen nur $40 \%$ angeregt und somit ist die skalierte Änderung $-6 \%$.

Generell kann man qualitativ festhalten, dass bei LBMO eine schwache Fluenz zum leichten Anstieg der Dichte der korrelierten Polaronen führt, wohingegen hohe Fluenzen diese verringern. Ähnliches Verhalten in der Fluenzabhängigkeit - allerdings in Bezug auf den Widerstand - beobachteten auch Moshnyaga et al. [Mos+04] bei ihren Untersuchungen der photoinduzierten Widerstandsänderung bei LCMO, wobei der Widerstand ihres dünnen Films unter Laseranregung bei Peakleistungen kleiner als $2.5 \cdot 10^{9} \mathrm{~J} / \mathrm{cm}^{2}$ um weniger als $1 \%$ ab-, aber darüber extrem stark zunahm. Nun passt die dort ermittelte Grenze in der Peakleistung für den Wechsel des Änderungsvorzeichens auch zu den hier diskutierten Ergebnissen von LBMO, aber der Sachverhalt ist gespiegelt: bei kleiner Leistung positive Änderung, bei großer negative.

Für LPCMO hingegen ist hier schon bei kleiner Leistung eine negative Änderung festzustellen, was damit erklärt werden kann, dass dort auch die Elektron-Phonon-Kopplung stärker und damit die Dichte korrelierter Polaronen höher ist. Die Wahrscheinlichkeit für

\footnotetext{
${ }^{5}$ Bei der Berechnung der Photonendichte wurde die Absorption der Manganate mithilfe des Absorptionskoeffizienten aus Gl. 2.15 berechnet. Es ist dabei ein durchschnittlicher Wert angegeben, da die Unterschiede aufgrund der anderen Komposition und Schichtdicke nicht besonders ausgeprägt sind.
} 
ein Photon, eine Polaron zu treffen, ist somit erhöht und wenn das System entsprechend empfindlicher auf diese Störung reagiert, wird der korrelierte Zustand geschwächt.

Mikroskopisch kann dabei eine Abnahme, wie sie in Greifswald für LBMO und in Göttingen bei LPCMO gefunden wurde, recht einfach verstanden werden: Wie schon in Kap. 2.3 beschrieben, existiert zu der Photonenenergie von $1.55 \mathrm{eV}$ ein elektronischer Übergang zwischen den beiden durch den Jahn-Teller-Effekt aufgespaltenen $e_{g}^{\uparrow}$-Niveaus. Dadurch ist durch die Umbesetzung des Orbitals die zugehörige Jahn-Teller-Verzerrung nicht mehr energetisch bevorzugt und der ausgedehnte Zustand der korrelierten Polaronen bricht zusammen. Natürlich müssen dafür genügend Polaronen gleichzeitig aufgebrochen werden, sodass es gut nachvollziehbar ist, warum eine hohe Fluenz benötigt wird, wie sie nur mit dem Aufbau in Greifswald realisierbar war.

Dem gegenüber ist die leichte Zunahme der Polaronendichte bei den Messungen an LBMO in Göttingen schwerer zu verstehen. Es könnte sich dabei um eine inkohärente Anregung typischer Jahn-Teller-Moden handeln, deren Energie jedoch eigentlich im Bereich von einigen zehn Millielektronenvolt liegt, weshalb sie auch im Normalfall mit infraroter Strahlung angeregt werden Rin+07. Doch über die Elektron-Phonon-Kopplung kann die Energie einer elektronischen Anregung ins Phononensystem dissipieren und dort die Moden anregen. Dass eine solche Anregung auch mit deutlich größeren Photonenenergien bei $1.55 \mathrm{eV}$ möglich ist, kann man bei RAISER et al. [Rai+17] sehen, die bei ihren zeitabhängigen Untersuchungen der optischen Dichte von $\overline{\mathrm{PCMO}}$ unter Laseranregung Oszillationen in der Relaxation finden, die sie kohärenten Phononenmoden zuschreiben. Ebenso zeigen sich die Anregungen dieser Moden bei BEAUd et al. Bea+14. Eine leichte Stimulation solcher Moden könnte also aufgrund der einfachen Beeinflussbarkeit des Systems in der Nähe des Phasenübergangs den korrelierten Zustand stabilisieren und so die Dichte korrelierter Polaronen erhöhen.

\subsubsection{Skalierung des gemessenen Effekts aufgrund zeitlicher Mittelung}

Wie bereits in Kap. 3.6 beschrieben, führt die vergleichbar lange zeitliche Mittelung des Lock-in-Verstärkers im Zusammenhang mit den kurzen Pulsdauern und den moderaten Repetitionsraten der Laser dazu, dass die Proben während der Signalaufnahme durch den Verstärker die meiste Zeit unbeleuchtet sind. Daher ist es nicht verwunderlich, dass trotz der hohen Pulsleistungen die erzielten Effekte - ausgenommen die Temperaturbeeinflussung - so gering sind. So beträgt der Anteil der Pulse an der Gesamtzeit beim Aufbau in Göttingen lediglich $9 \cdot 10^{-6}$ und in Greifswald sogar nur $1.1 \cdot 10^{-8}$, was bedeutet, dass bei ultraschneller Reaktion und Relaxation der Probe eine Änderung des Signals nicht messbar gewesen wäre.

Allerdings ist die Lebenszeit der Anregung entscheidend, denn je nachdem, wie lange diese ist, fällt das durch den Lock-in-Verstärker aufgezeichnete Signal größer oder kleiner aus. In jedem Fall repräsentiert die gemessene Änderung im Signal nur einen zeitlich gemittelten und damit deutlich kleineren Wert als die ursprünglich ausgelöste Veränderung. Bei einer instantanen Antwort der Probe und sofort einsetzender, langsamer Relaxation ergäbe sich als gemessene Änderung die aufsummierte Fläche unter einer 


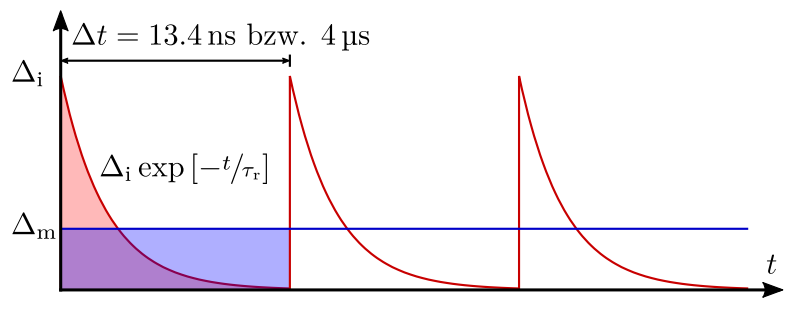

Abbildung 5.6.: Zeitlicher Verlauf der Signaländerung aufgrund der Laseranregung. Vereinfacht wird angenommen, dass die anfängliche Änderung $\Delta_{i}$ bei Eintreffen eines Pulses instantan geschieht. Der Lock-inVerstärker misst nur die zeitliche Mittelung $\Delta_{\mathrm{m}}$.

Exponentialfunktion, die sich im Lock-in-Verstärker als Mittelung $\Delta_{\mathrm{m}}$ über die gesamte Zeit verteilt (Abb. 5.6). Um nun die ursprüngliche Amplitude $\Delta_{i}$ der Änderung zu ermitteln, müssen die Flächen ins Verhältnis gesetzt werden

$$
\Delta_{\mathrm{m}} \cdot \Delta t=\int_{0}^{\Delta t} \Delta_{\mathrm{i}} \exp \left[-t / \tau_{\mathrm{r}}\right] \mathrm{d} t,
$$

woraus sich der Skalierungsfaktor $S$

$$
\Delta_{\mathrm{i}}=\underbrace{\frac{\Delta t}{\tau_{\mathrm{r}}\left(1-\exp \left[-\Delta t / \tau_{\mathrm{r}}\right]\right)}}_{=: S} \cdot \Delta_{\mathrm{m}}=S \cdot \Delta_{\mathrm{m}}
$$

ergibt, wobei $\Delta t$ der zeitliche Abstand zwischen den Pulsen und $\tau_{\mathrm{r}}$ die Relaxationszeit ist. Dabei ist der exponentielle Term im Nenner nur wichtig, wenn $\tau_{\mathrm{r}} \sim \Delta t$, denn ansonsten ergibt sich die Skalierung einfach aus dem Verhältnis $\Delta t / \tau_{\mathrm{r}}$.

Nun ist es nicht trivial, für Anregungen in Manganaten eine typische Relaxationszeit anzugeben, da sich die charakteristischen Zeitskalen über etliche Größenordnungen erstrecken. So gibt es Vorgänge wie z. B. die Änderung der Reflektivität, die im Bereich von Subpikosekunden $[\mathrm{Wu}+09$ bis einige zehn Pikosekunden [Dor+05] relaxieren kann, oder laserinduzierte Leitfähigkeit, bei der eine Komponente in $\sim 150$ ps relaxiert [Zha+98], und schließlich Phänomene, die von der Spindynamik abhängen, wie u.a. die Magnetisierungsänderung, mit Zeitskalen von Nano- oder Mikrosekunden Oga+03. Ren+08, Ren+04, Fie+00, Mül+09]. Je nachdem, welche Relaxationszeit man zugrunde legt, ergibt sich ein anderer Faktor, mit welchem man den gemessenen Effekt $\Delta_{\mathrm{m}}$ skalieren muss, um die ursprünglich Veränderung $\Delta_{\mathrm{i}}$ zu erhalten. Dafür ist in Abb. 5.7 der Skalierungsfaktor in Abhängigkeit einer möglichen Relaxationszeit aufgeführt. Jedoch ist ein enorm großer Faktor für sehr kurze Relaxationszeiten physikalisch nicht mehr sinnvoll. Jetzt ist aus den Messungen von GeHRKe et al. Geh+10 bekannt, dass sich $K_{3 \omega}$ durch statische Modifikation einer Übergitterstruktur mittels Einfügen einiger Zwischen-Monolagen von $10^{-1}$ auf $10^{-4}$ unterdrücken lässt (s.a. Abb. 2.10), was einer Änderung des Signals um $-99.9 \%$ entspricht. Geht man von dieser Änderung ebenfalls als initialem Effekt $\Delta_{i}=-99.9 \%$ in unseren Messungen aus, so kann man eine Relaxationszeit von $\tau_{\mathrm{r}} \sim 150 \mathrm{~ns}$ bestimmen, die dann zu der von uns beobachteten, zeitlich gemittelten Änderung von $\Delta_{\mathrm{m}}=-3.75 \%$ führt. Eine Zeitskala gleicher Größenordnung fanden auch FIEBIG et al. Fie+00] in PCMO bei der Relaxation der Reflektivität nach 


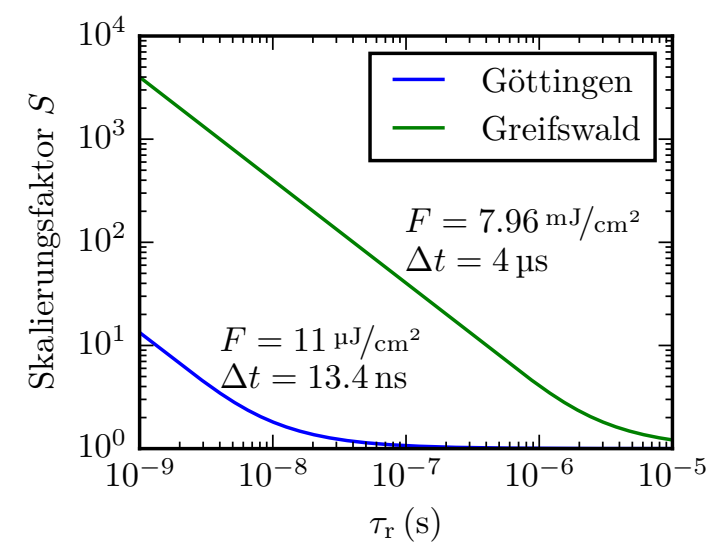

Abbildung 5.7.: Skalierungsfaktor $S$ für den gemessenen Effekt bei verschiedenen Relaxationszeiten (s. Gl. (5.3)). Für sehr kurze Relaxationszeiten ergeben sich riesige Faktoren, die physikalisch nicht mehr sinnvoll sind, gerade für eine negative Änderung bei den Messungen in Greifswald. Geht man von vollständiger Auslöschung $\Delta_{\mathrm{i}}=-99.9 \%$ aus, so wäre bei einer gemessenen Änderung von $\Delta_{\mathrm{m}}=-3.75 \%$ ein Skalierungsfaktor von 26.6 nötig, was zu $\tau_{\mathrm{r}} \sim 150 \mathrm{~ns}$ führt.

Lichtanregung zurück in ihren ursprünglichen Zustand. Weiterhin spricht diese langsame Relaxationszeit auch eher für strukturelle Änderungen als für rein elektronische.

Wenn man jetzt LBMO und LPCMO unter Einfluss der geringen Laserfluenz betrachtet und für diesen Fall ebenso die gerade erhaltene Relaxationszeit von $\tau_{\mathrm{r}} \sim 150 \mathrm{~ns}$ annimmt, so kann man umgekehrt die dortige initiale Änderungen $\Delta_{i}$ bestimmen. Unter Berücksichtigung, dass hier $\tau_{\mathrm{r}} \sim 11 \Delta t$ ist, berechnet man einen nur leicht erhöhten tatsächlichen Effekt von $\Delta_{\mathrm{i}}=+3.1 \%$ bei LBMO und $\Delta_{\mathrm{i}}=-6.3 \%$ bei LPCMO. Und aufgrund der fast drei Größenordnungen kleineren Anregungsfluenz entspricht dieses nahezu den gemessenen Werten.

\subsection{Abhängigkeit von der Anregungsposition}

Die Untersuchung der harmonischen Spannungen in Abhängigkeit von der Anregungsposition (Kap. 4.1.6) bietet eine Möglichkeit, die Reaktion der Probe bei Anregung auf und neben dem Steg zu betrachten. Dafür zeigt Abb. 4.14 eine Messung, bei welcher der Laser einmal über den Steg und einmal über die Kontakte gefahren wurde. Da die äußere Temperatur auf $288 \mathrm{~K}$ eingestellt wurde, ist generell davon auszugehen, dass sich aufgrund der Erwärmung durch den Laser die Temperatur der Probe oberhalb des Übergangs und damit des Maximums befindet. Da dort $\mathrm{d} R / \mathrm{d} T<0$ ist, bedeutet ein zunehmender Widerstand, welcher proportional zum gemessenen $U_{\omega}$ ist, eine Abnahme der Probentemperatur, wenn man davon ausgeht, dass der größte Teil einer durch den Laser verursachten Widerstandsänderung durch Temperatureinfluss zustande kommt.

So fällt dann auch die Temperatur ab, wenn man vom umliegenden Vollfilm auf das Substrat in den $400 \mu \mathrm{m}$ breiten, strukturierten Bereich 6 gelangt, was offensichtlich bedeutet, dass die höhere Absorption durch den Vollfilm den Steg stärker erwärmt als die Absorption des \$TO-Substrates, obwohl man sich dem Steg nähert. Beim Scan über die Kontakte ist der Temperaturabfall noch größer, da natürlich die Goldkontakte das meiste der Laserleistung reflektieren. Befindet man sich dann in der Nähe des Steges,

\footnotetext{
${ }^{6}$ Die Probengeometrie findet man in Abb. 4.4
} 
steigt die Temperatur wieder, da durch das Manganat wieder mehr Licht absorbiert werden kann. Jedoch beleuchtet ein Teil des $80 \mu \mathrm{m}$ breiten Strahls immer noch das Substrat, sodass nicht die Temperaturhöhe erreicht wird, die man auf dem Vollfilm beobachten konnte. Bemerkenswert ist noch, dass man den Steg auch ausmachen kann, während über den Kontakt gefahren wird, was vielleicht bedeuten kann, dass der Steg dennoch teilweise getroffen wurde. Anschließend ist der weitere Verlauf im Wesentlichen symmetrisch zum Vorausgegangenen.

Gleichzeitig folgt die dritte harmonische Spannung $U_{3 \omega}$ qualitativ dem Verlauf von $U_{\omega}$, wobei Erstere aufgrund ihres kleinen Signales deutlich verrauschter ist und beim Scan über die Kontakte eine Oszillation sichtbar wird. Doch die Verringerung des Signals in $U_{3 \omega}$, wenn die Laseranregung auf Steg ist, ist etwas größer, als man bei derselben Messung in $U_{\omega}$ beobachtet, was dementsprechend durch den photoinduzierten Effekt hervorgerufen sein kann.

Bei der zweiten Messung zur Positionsabhängigkeit, die Abb. 4.15 zeigt, wurden für verschiedene Anregungspositionen jeweils komplette Temperaturkurven der ersten und dritten harmonischen Spannung aufgenommen und dafür Temperaturverschiebung durch Wärmeeintrag und die relative Änderung des dritten harmonischen Koeffizienten ermittelt. Dabei ergibt sich für die Temperatur ein stimmiges Bild zur zuvor vorgestellten Messung, denn das Heizen der Probe durch den Laser ist auch hier auf dem unstrukturierten Vollfilm stärker als auf dem Substrat oder auf dem Steg selbst. Auch quantitativ sind die Verschiebungen vergleichbar, da sich beim Umrechnen von $U_{\omega}$ aus Abb. 4.14 weit entfernt vom Steg eine Temperaturänderung von $\Delta T \sim 17 \mathrm{~K}$ und auf dem Steg $\Delta T \sim 12 \mathrm{~K}$ ergibt.

Hier wurde allerdings für den dritten harmonischen Koeffizienten bei LBMO als einzige von den Messungen in Greifswald eine Erhöhung des Messwertes festgestellt. Jedoch sind die Fehler aufgrund stark verrauschter Messdaten (s. Abb. A.2 im Anhang) sehr groß, sodass eher nur das Vorzeichen der Messung bewertet werden sollte. Dafür kann es sein, dass der Steg beim Rastern nicht genau getroffen wurde und lediglich Streulicht von der Rückseite der Probe, auf der Leitsilber zur Haftung auf dem Probenhalten verwendet wurde, den Steg traf. Die somit geringe Intensität würde wieder zu der beobachten Erhöhung der Polaronendichte führen. 



\section{Zusammenfassung und Ausblick}

In der hier vorliegenden Arbeit wurden die Auswirkungen von Laseranregungen mit ultrakurzen Pulsen auf die elektronischen Transporteigenschaften in Manganaten mit starken Korrelationen untersucht, wobei im Besonderen die Nichtlinearitäten von Interesse waren. Dabei ist nämlich die sogenannte dritte harmonische Spannung bei einer Wechselstrommessung ein Maß für die Dichte korrelierter Polaronen.

Da Letztere jedoch temperaturabhängig und am Metall-Isolator-Übergang am ausgeprägtesten ist, war es notwendig, eine Möglichkeit zur Kontrolle der Temperatur während der Messungen zu schaffen. Dafür wurde ein Messeinsatz mit Führung des Laserlichts bis zur Probe für einen kommerziellen Großkryostaten konzipiert, entworfen und konstruiert, sodass die Bedingungen für Temperatur und Magnetfeld auf einfache weise von außen einstellbar waren. In diesem Zusammenhang stellte sich als besondere Herausforderung der geringe Platz im Kryostaten und eine Vakuumdurchführung für den speziellen Lichtwellenleiter des Lasers dar. Später wurde noch ein zweiter Probenhalter mit begrenzter Kühlmöglichkeit für direkte Messung mit Freistrahl auf dem optischen Tisch gebaut, welcher dann auch in Verbindung mit einem stärkeren Lasersystem in Greifswald zum Einsatz kam.

Mithilfe dieser Messaufbauten wurden zwei Manganatproben untersucht: zum einen Lanthan-Barium-Manganat (LBMO), welches eine Übergangstemperatur nahe Raumtemperatur besitzt, jedoch nur moderat ausgeprägte Korrelationen bzw. ElektronPhonon-Wechselwirkung aufweist; zum anderen Lanthan-Praseodym-Calcium-Manganat (LPCMO) mit deutlich tieferer Übergangstemperatur, aber dafür starker ElektronPhonon-Kopplung. An strukturierten Stegen dieser Proben wurde dann, während man ihnen einen konstanten Wechselstrom aufgeprägte, die über der Probe abfallenden Spannungen auf der ersten und auf der dritten harmonischen Frequenz als Funktion der Zeit aufgezeichnet und dabei die Laseranregung mit ultrakurzen Pulsen auf dem Steg einund abgeschaltet. Dieses geschah bei verschiedene Temperaturen, sodass eine Temperaturkurve der ersten und dritten harmonischen Spannung mit und ohne Laserlicht erstellt werden konnte, woraus sich schlussendlich die laserinduzierte Änderung bestimmen lies.

Dabei konnte man erkennen, dass für LBMO bei Anregung mit geringer Laserfluenz eine Zunahme der Dichte korrelierter Polaronen stattfand, bei einer sehr viel höheren Fluenz jedoch die Polaronendichte deutlich abnahm. Die LPCMO-Probe hingegen, die aufgrund der niedrigen Übergangstemperatur lediglich am Aufbau in Göttingen untersucht werden konnte, zeigte auch schon bei der hier vorhandenen kleineren Laserleistung eine negative Änderung [1] Jetzt kann die Abnahme der Polaronendichte gut mit einer elektronischen Anregung zwischen den Jahn-Teller-aufgespaltenen $e_{g}$-Niveaus erklärt

\footnotetext{
${ }^{1}$ Es sei erwähnt, dass das Auftreten eines vorher nicht vorhandenen Merkmals im Widerstand nach
} 
werden, da dieses zum Aufbrechen der Polaronen und damit zum Verlust des korrelierten Zustandes führt. Schwieriger zu erklären ist die Zunahme korrelierter Polaronen, wofür vielleicht eine inkohärente Anregung der Jahn-Teller-Moden infrage kommt. Diese sind zwar energetisch mehr als eine Größenordnung unterhalb der hier verwendeten Photonenenergie, bei geringer Anregung ist es aber eventuell möglich, die Moden zu stimulieren, was dann zu einer Bevorzugung der Korrelationen führen würde.

Für den positiven und negativen Effekt gleichermaßen gilt jedoch, dass aufgrund der gepulsten Anregung mit dem Laser die Probe den größten Teil der Zeit unbeleuchtet ist, was bei einer moderaten Relaxationszeit dazu führt, dass das System nur kurz im angeregten Zustand ist. Da die elektronische Messung jedoch dauerhaft stattfindet, erhält man einen zeitlich gemittelten Wert und nicht die ursprüngliche Anregung. Dennoch ist die Änderung messbar, was dafür spricht, dass die anfängliche Effekt direkt nach der Anregung deutlich größer ist. Geht man von bekannten Änderungen in der Polaronendichte aus, so kann man eine Lebensdauer der Anregung von 150 Nanosekunden ermitteln.

Weiterhin wurden Strom-Spannungs-Kennlinien von LBMO bei verschiedenen Temperaturen betrachtet, wobei sich ein starker nicht linearer Zusammenhang offenbarte. Hierfür konnten einfache Heizeffekte ausgeschlossen werden und die Analysen im Zusammenhang mit Untersuchungen in der Literatur legen nahe, dass ein spannungsaktivierter Prozess das phasenseparierte System in einen transienten metallischen Zustand treibt.

Außerdem wurde mithilfe modulierter Anregung die Wärmedissipation untersucht und dabei zwei deutlich unterschiedliche Zeitskalen gefunden, die gerade durch den Metall-Isolator-Übergang getrennt sind. Die längere Relaxationszeit fand man in der ferromagnetischen metallischen Phase, bei der die Dissipation über die Spinfreiheitsgrade stattfindet, deren Kopplung an das Elektronensystem langsam ist.

Weitere Untersuchungen könnten ein umfassenderes Bild der hier angetroffenen Phänomene zeichnen. So wäre in jedem Fall interessant, ob und wie der Effekt der Polaronenvernichtung mit der Laserleistung skaliert. Ebenso kann man den Einfluss der Stegbreite betrachten. Und schließlich könnte man mit einer Autokorrelationsmessung, wie sie WANG et al. Wan+08 an Photoschaltern durchführten, die hier nur abgeschätzte Zeitskala genauer untersuchen und zusätzlich überprüfen, ob auf sehr kurzen Zeitskalen auch noch Anregungen relaxieren. Dabei wäre für die Betrachtung der langsamen Zeitskala eine stufenweise Änderung der Repetitionsrate vom Laser ausreichend, bei den kurzen Skalen könnte man die Pulse teilen und mithilfe einer Verzögerungsstrecke gegeneinander verschieben. Natürlich kann der hier entwickelte Messaufbau für jegliche elektrische Messung von dünnen Proben verwendet werden, bei denen der Einfluss von Laseranregung mit kurzen Pulse im Nahinfraroten interessant ist, während Temperatur und magnetisches Feld für einen großen Bereich frei gewählt werden können.

der Strukturierung dieser Probe eine zweite Phase mit erniedrigter Übergangstemperatur nahelegt, bei der die Nichtlinearitäten noch stärker hervortreten. Aus diesem Grund sollte dort auch die Elektron-Phonon-Wechselwechselwirkung noch mehr erhöht sein, was erklärt, warum es schon bei der geringen Laserleistung zu einer negativen Änderung kommt. 


\section{A. Anhang}

\section{A.1. Zusätzliche Graphen}

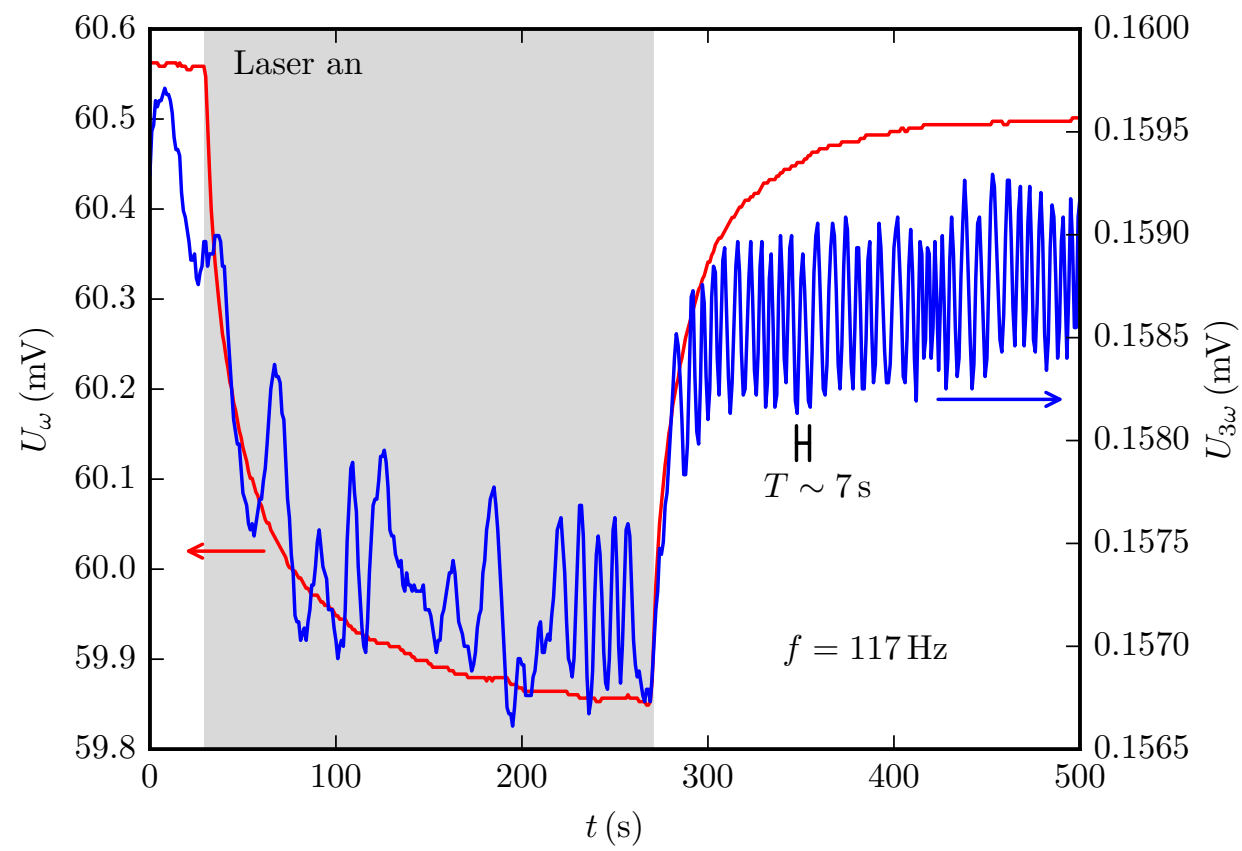

Abbildung A.1.: Schwingverhalten in der dritten harmonischen Spannung bei Wechselstromanregung mit $f=117 \mathrm{~Hz}$. Das Signal oszilliert mit einer Periodendauer von $T \sim 7 \mathrm{~s}$. Ergänzung zu den Graphen aus Kap. 4.1 .2 


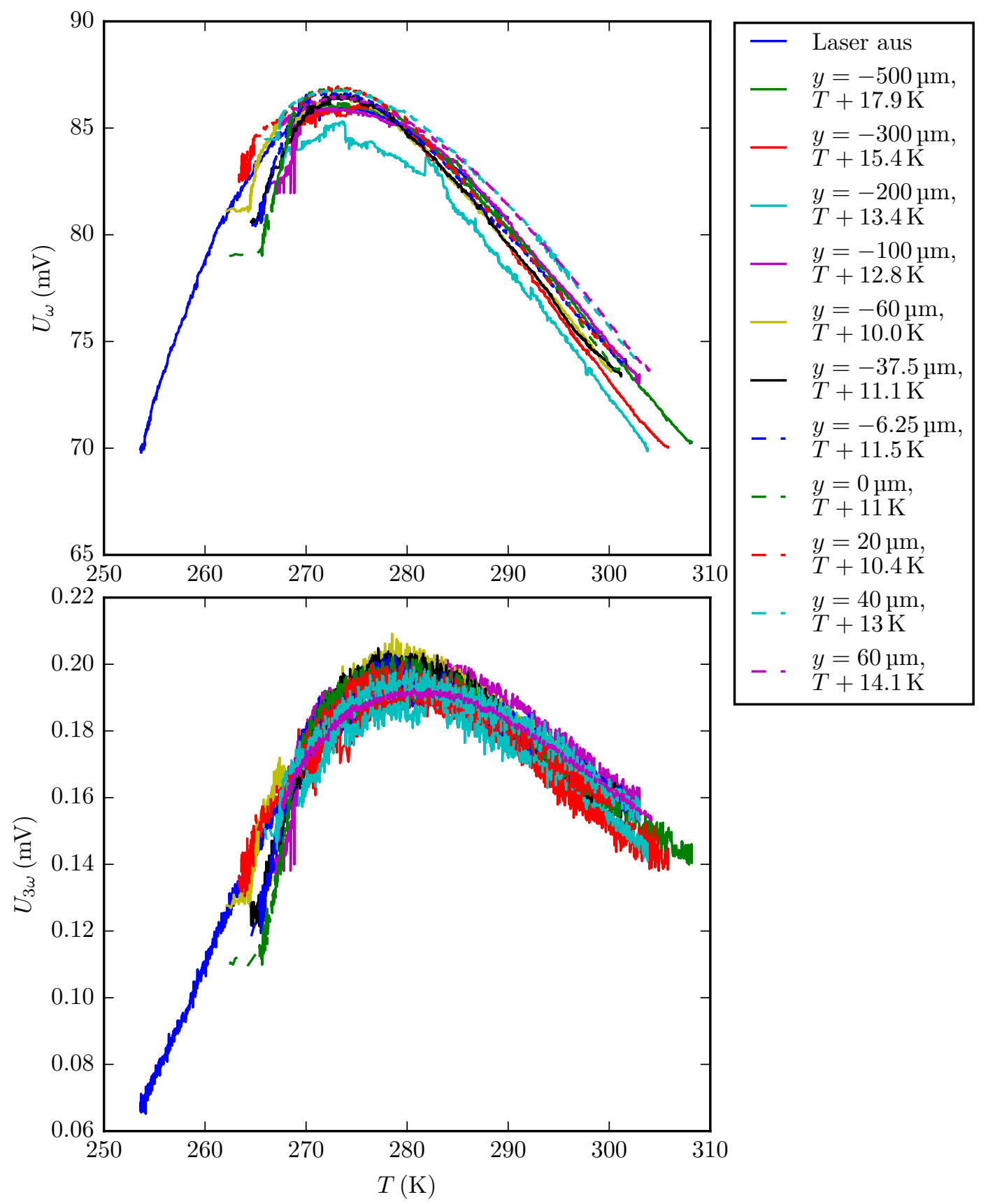

Abbildung A.2.: Temperaturabhängigkeit der ersten und dritten harmonischen Spannung von LBMO bei positionsabhängiger Laseranregung in Greifswald. Die Maxima wurden jeweils so korrigiert, dass sie mit dem der nicht beleuchteten Probe zusammenfallen, woraus sich die Temperaturverschiebungen ergeben. Für jede Position konnte dann der Wert des Maximums bestimmt werden, wie er in Abb. 4.15 gezeigt ist. Aufgrund des starken Rauschens weisen die extrahierten Werte einen großen Fehler auf. 

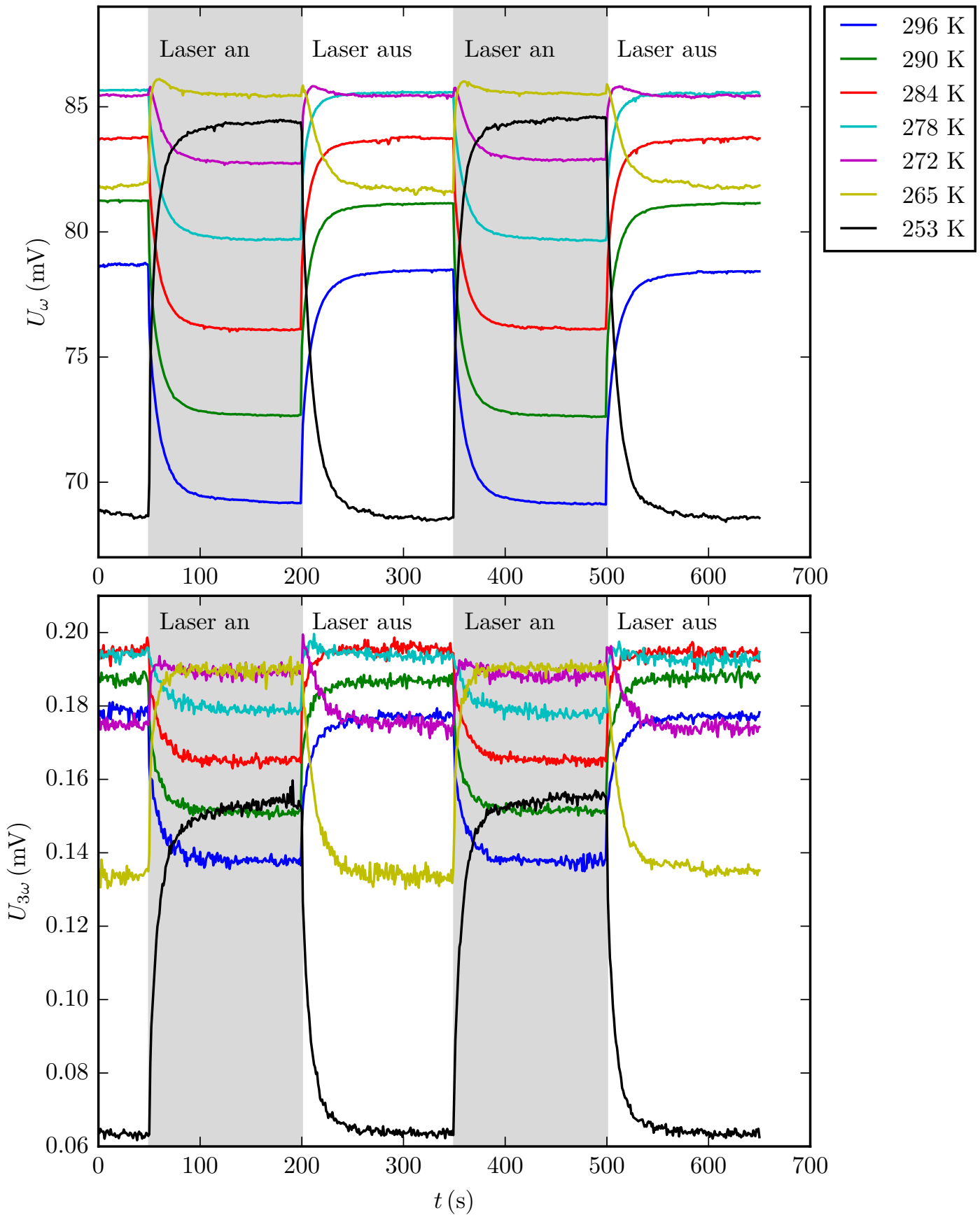

Abbildung A.3.: Zeitserien der LBMO-Probe mit Laseranregung aus Greifswald für ausgewählte Temperaturen. Die aus den Plateauwerten extrahierten temperaturabhängigen Kurven zeigt Abb. 4.13 . 


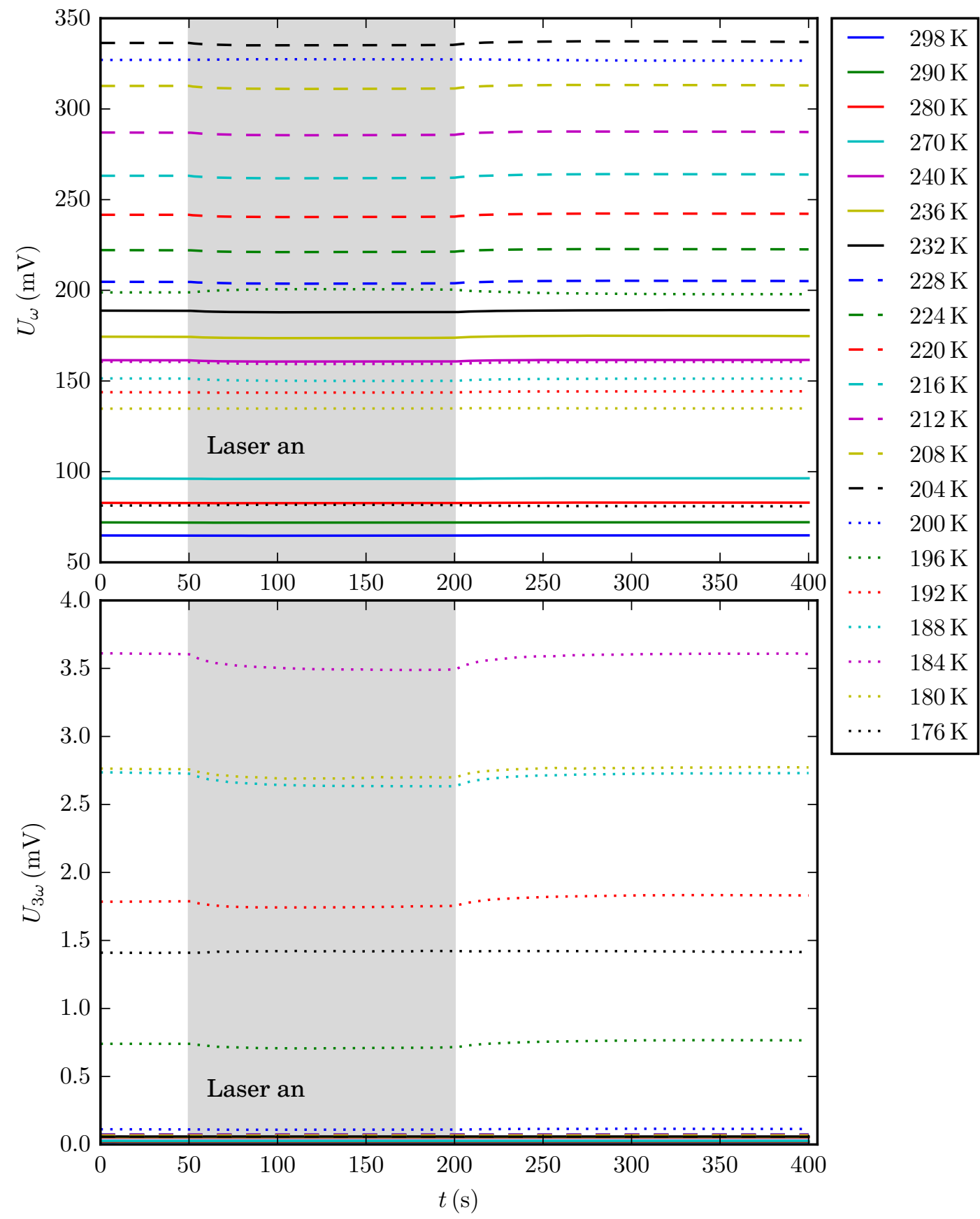

Abbildung A.4.: Zeitserien der LPCMO-Probe mit Laseranregung für ausgewählte Temperaturen. Die aus den Plateauwerten extrahierten temperaturabhängigen Kurven zeigt Abb. 4.24 

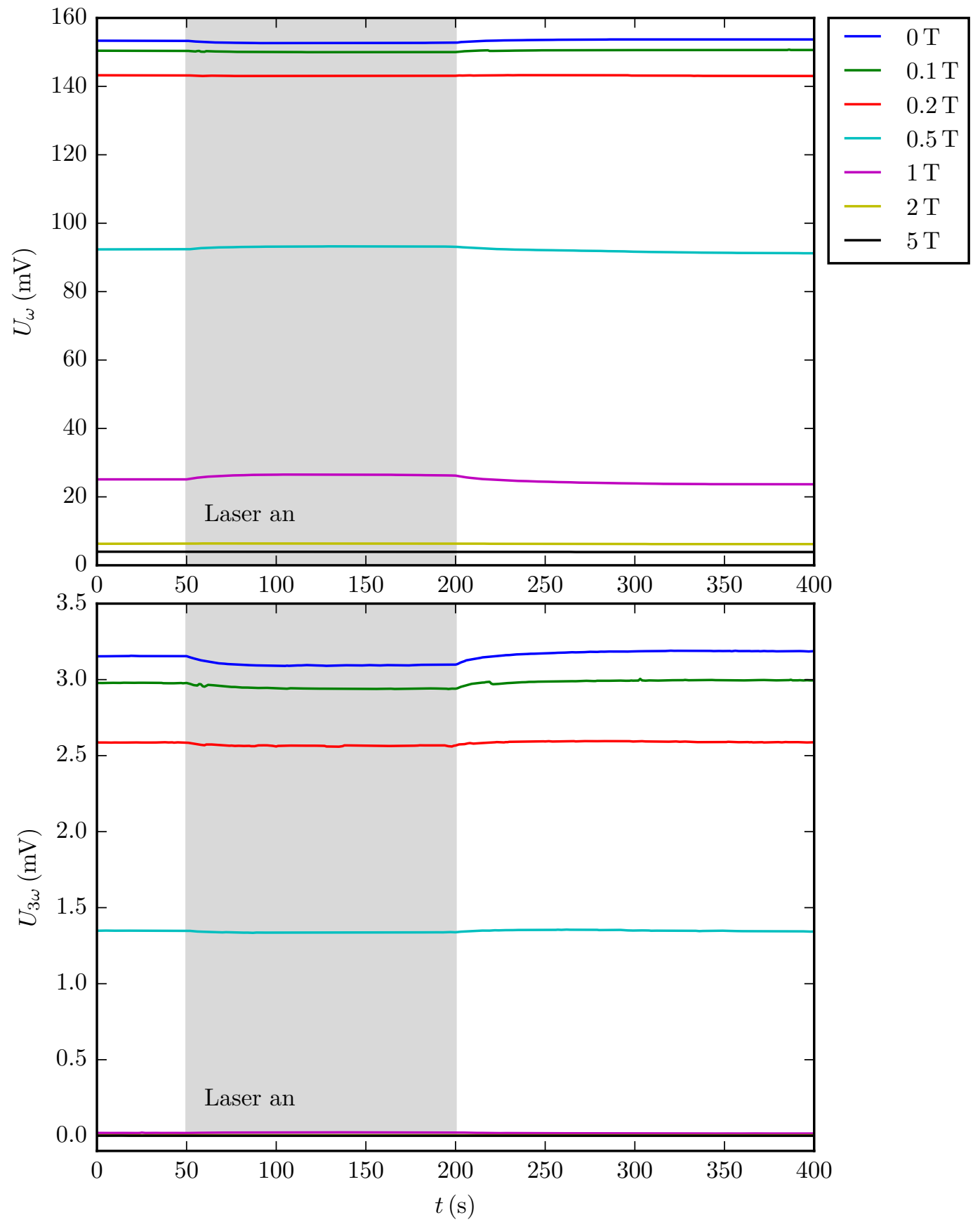

Abbildung A.5.: Zeitserien der LPCMO-Probe mit Laseranregung beim Anlegen eines Magnetfeldes. Die aus den Plateauwerten extrahierten Kurven zeigt Abb. 4.26 

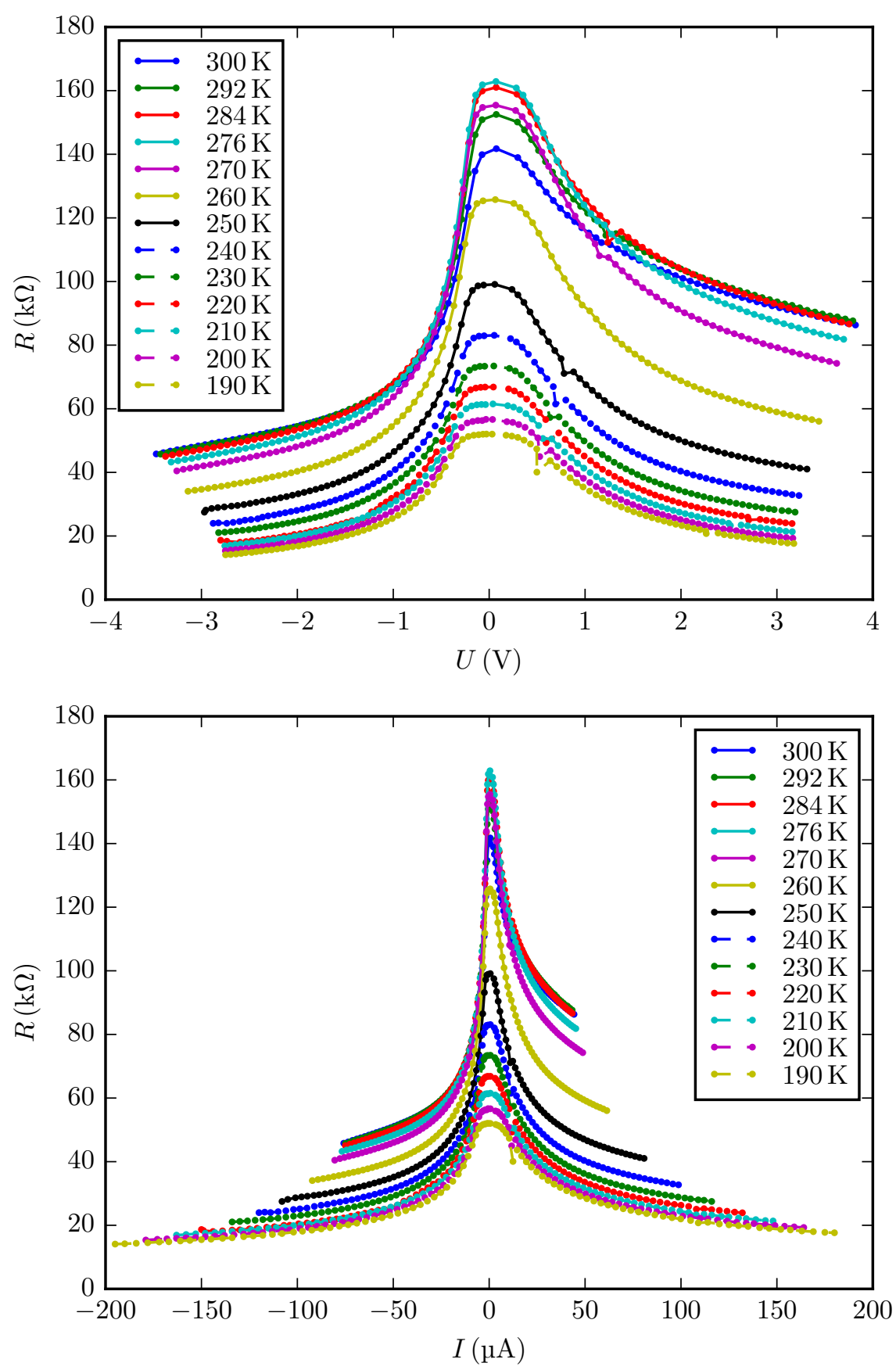

Abbildung A.6.: Widerstand in Abhängigkeit von Strom und Spannung von LBMO für ausgewählte Temperaturen, extrahiert aus den Strom-Spannungs-Kennlinien aus Abb. 4.17. Logarithmische Auftragung der negativen Bereiche in Abb. A.7. 

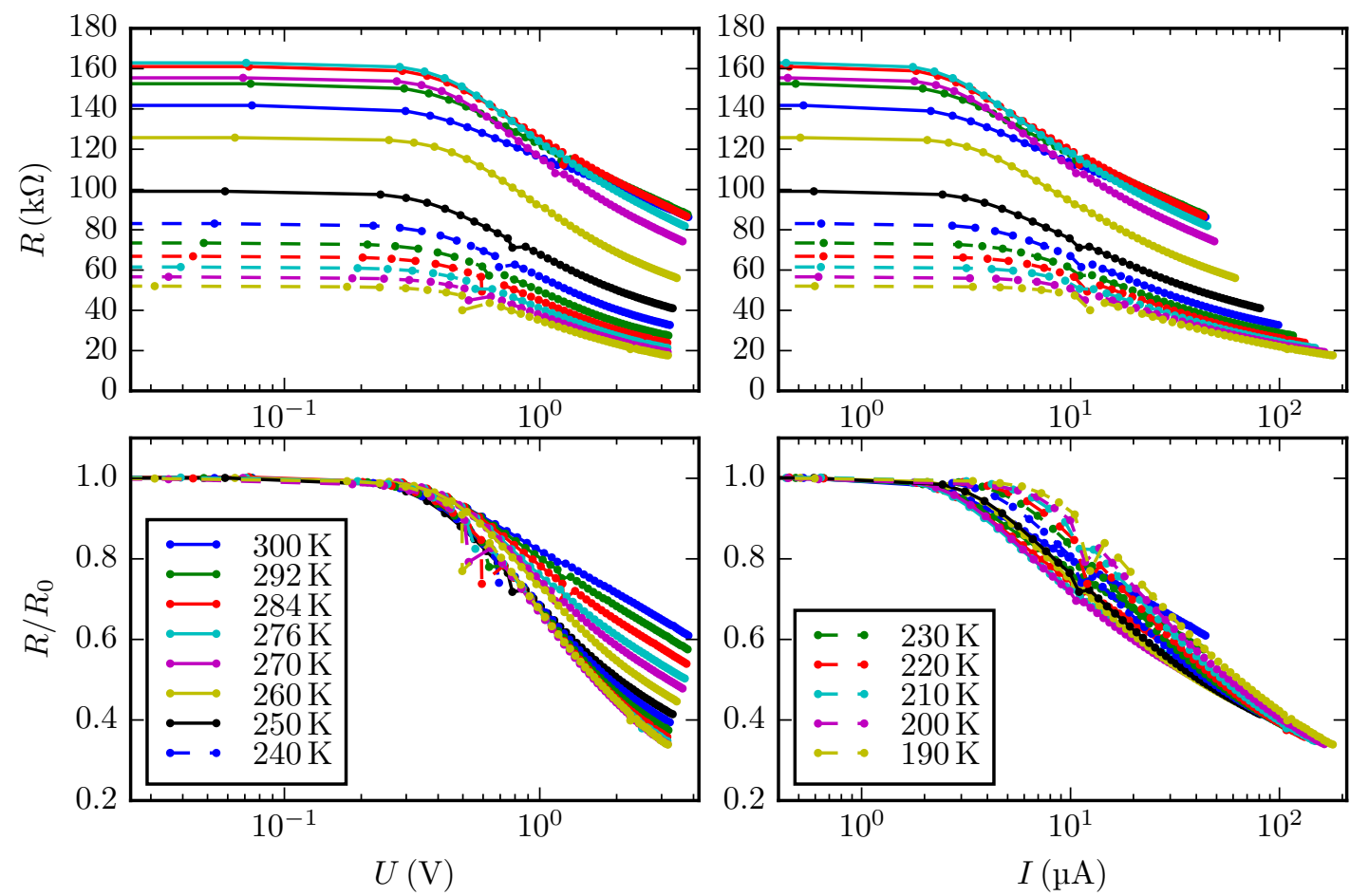

Abbildung A.7.: Widerstand in Abhängigkeit von Strom und Spannung von LBMO für ausgewählte Temperaturen, Logarithmische Auftragung des positiven Astes, bestimmt aus den Strom-Spannungs-Kennlinien aus Abb. 4.17 Vollständige Kurven in Abb. A.6 negativer Ast in Abb. 5.4 

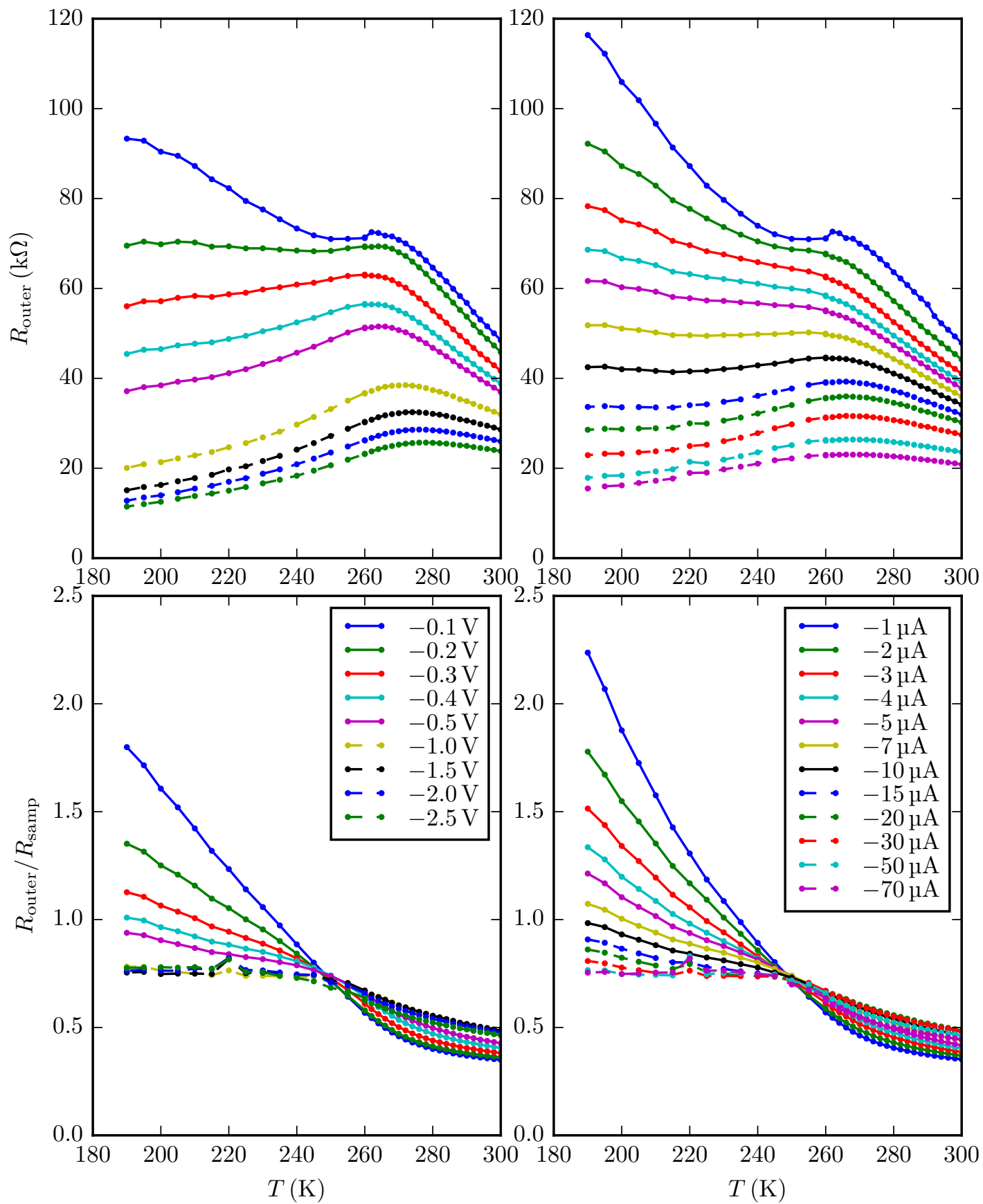

Abbildung A.8.: Widerstände der äußeren Kontakte bei der Vierpunktmessung an LBMO, bei den Strom-Spannungs-Kennlinien bestimmt aus der Spannungsdifferenz zwischen äußeren und inneren Kontakten, wobei letztere den Probenwiderstand repräsentieren. Dort enthalten sind die Widerstände der elektrischen Zuleitungen, der Kontakte und ein kleiner Teil Manganat bis zu einem der inneren Kontakte. Es handelt sich um die Summe aus beiden Kontakten. 


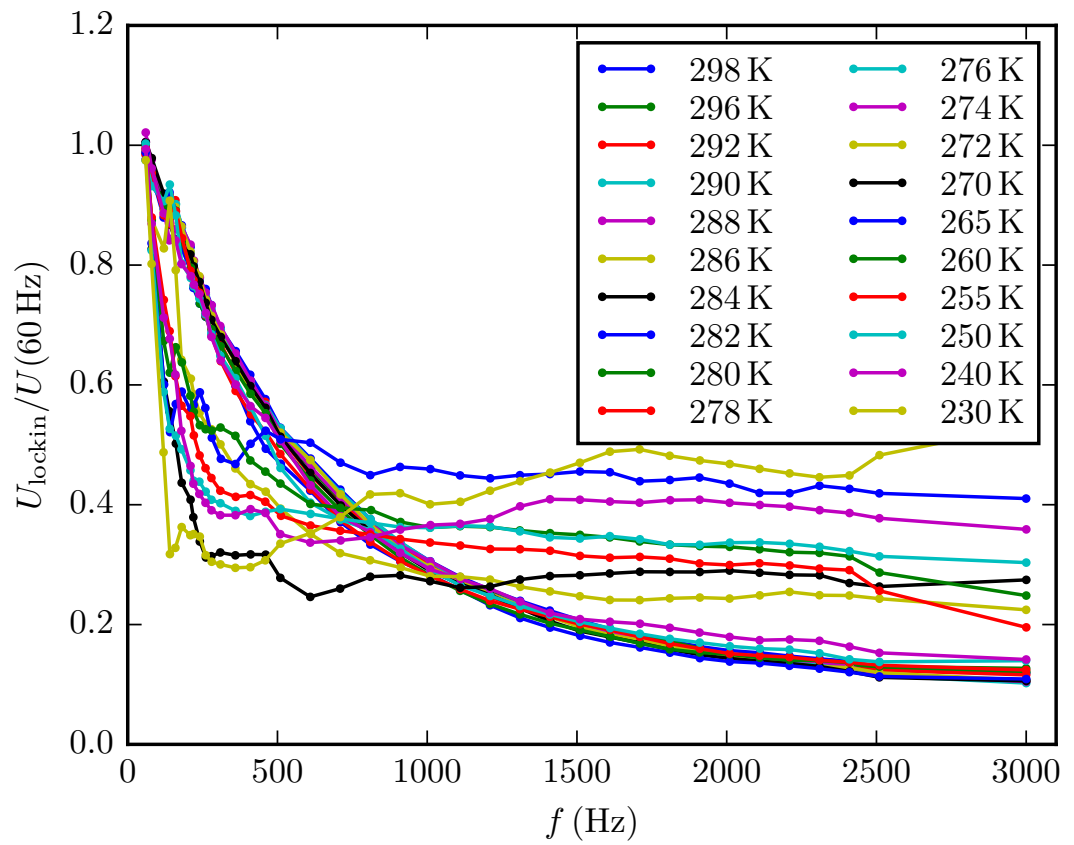

Abbildung A.9.: Normierte Änderung des Gleichstromwiderstandes bei periodischer Laseranregung, Daten leicht geglättet. Die Form der Kurven unterscheidet sich deutlich zwischen $T \geq 274 \mathrm{~K}$ und $T \leq 272 \mathrm{~K}$. Damit müssen zwei unterschiedlich schnelle Prozesse zugrunde liegen. Eine mögliche Intensitätsminderung der Laserleistung durch sich bildendes Eis kann damit ausgeschlossen werden. 


\section{A.2. Abgrenzung der Messmethode}

Die in dieser Arbeit verwendete Messmethode soll kurz der auf den ersten Blick identischen $3 \omega$-Messtechnik zur Bestimmung der thermischen Leitfähigkeit von dielektrischen Materialien, wie sie CAHILL Cah90 beschreibt, gegenübergestellt werden, um sie davon abzugrenzen. So wird dort auf eine zu untersuchende Probe ein dünner Steg aus einem Metall aufgebracht und dieser durch einen Wechselstrom $I(t)=I_{0} \cos (\omega t)$ durchflossen. Dabei kommt es aufgrund der Widerstandes im Material zu einer jouleschen Aufwärmung mit der Leistung

$$
P_{\text {heiz }}=I^{2} R=I_{0}^{2} R \cos ^{2}(\omega t)=I_{0}^{2} R \cdot[1+\cos (2 \omega t)]
$$

bei der doppelten Frequenz. Nun ist die Temperaturänderung proportional zur Heizleistung $\Delta T \propto P_{\text {heiz }}$ und der Widerstand in Metallen hängt bei nicht allzu tiefen Temperaturen linear von der Temperatur ab, womit auch die Widerstandsänderung proportional zur Temperaturänderung ist. Folglich erhält man

$$
\Delta R \propto \Delta T \propto P_{\text {heiz }} .
$$

Wenn man jetzt den Spannungsabfall über dem Metallsteg misst, bekommt man dort

$$
U(t)=R I=\left(R_{0}+\Delta R\right) I=R_{0} I_{0} \cos (\omega t)+\Delta R I_{0} \cos (\omega t)
$$

und somit über

$$
\Delta R I_{0} \cos (\omega t) \propto \cos ^{2}(\omega t) \cdot \cos (\omega t)=3 / 4 \cos (\omega t)+1 / 4 \cos (3 \omega t)
$$

einen Beitrag zum Signal bei der dritten harmonischen Frequenz.

Dieses sieht sehr ähnlich zu der hier verwendeten und in Kap. 2.2 hergeleiteten Messmethode aus, weist jedoch wichtige Unterschiede auf. Denn es handelt sich bei dem Steg hier um kein Metall, sondern um ein Manganat, welches keine einfache lineare Temperaturabhängigkeit im Widerstand zeigt, sondern deutlich komplizierteres Verhalten mit einem Maximum. So würde man nach der hier gezeigten Beschreibung das größte Messsignal in $U_{3 \omega}$ bei $T_{\mathrm{TCR}}$ erwarten, da dort die Steigung $\mathrm{d} R / \mathrm{d} T$ und damit der lineare Anteil maximal werden, und nicht in der Nähe des Widerstandsmaximums, wo $\mathrm{d} R / \mathrm{d} T$ gerade verschwindet. Tatsächlich findet man aber für LBMO das Maximum in $U_{3 \omega}$ bei $T_{3 \omega, \max } \sim 286 \mathrm{~K}$ und damit in der Nähe des Übergangs $T_{\mathrm{MI}} \sim 280 \mathrm{~K}$ und nicht bei $T_{\mathrm{TCR}} \sim 258 \mathrm{~K}$. Ebenso ist bei LPCMO $T_{3 \omega, \max } \sim 184 \mathrm{~K}$, was näher zum zweiten Maximum bei $T_{2 \text { nd }} \sim 182 \mathrm{~K}$ liegt als zu $T_{\mathrm{TCR}} \sim 196 \mathrm{~K}$. Somit kann ein Einfluss der dort beschriebenen Zusammenhänge auf die hier ermittelten Messdaten praktisch ausgeschlossen werden. 


\section{Literatur}

[AB99] A. S. Alexandrov und A. M. Bratkovsky. „Carrier Density Collapse and Colossal Magnetoresistance in Doped Manganites". In: Phys. Rev. Lett. 82, 141-144 (1999). DOI: 10.1103/PhysRevLett.82.141.

[And50] P. W. Anderson. „Antiferromagnetism. Theory of Superexchange Interaction“. In: Phys. Rev. 79, 350-356 (1950). DOI: 10.1103/PhysRev.79.350.

[Asa+97] A. Asamitsu, Y. Tomioka, H. Kuwahara und Y. Tokura. „Current switching of resistive states in magnetoresistive manganites". In: Nature 388, 50-52 (1997). DOI: 10.1038/40363.

[Bar+00] S. Barilo et al. „Seeded growth from flux and neutron study of $\mathrm{La}_{1-x} \mathrm{Ba}_{x}$ $\mathrm{MnO}_{3}(0.2<x<0.5)$ single crystals". In: J. Cryst. Growth 211, 480-484 (2000). DOI: $10.1016 / \mathrm{S} 0022-0248$ (99)00767-8.

[BB48] J. Bardeen und W. H. Brattain. „The Transistor, A Semi-Conductor Triode“. In: Phys. Rev. 74, 230-231 (1948). DOI: 10.1103/PhysRev.74.230.

$[$ Bea +14$]$ P. Beaud et al. „A time-dependent order parameter for ultrafast photoinduced phase transitions“. In: Nat. Mater. 13, 923-927 (2014). DOI: $10.1038 /$ nmat 4046

[Bec+02] T. Becker et al. „Intrinsic Inhomogeneities in Manganite Thin Films Investigated with Scanning Tunneling Spectroscopy". In: Phys. Rev. Lett. 89, 237203 (2002). DOI: 10.1103/PhysRevLett.89.237203.

[BJM15] C. Ballani, M. Jungbauer und V. Moshnyaga. „Report on processing of $\mathrm{Si} / \mathrm{STO} / \mathrm{LMO} / \mathrm{SMO}$ heterostructures". Interfacing Oxides (IFOX) - Final Report. 2015.

[BM14] A. Bhattacharya und S. J. May. „Magnetic Oxide Heterostructures“. In: Annu. Rev. Mater. Res. 44, 65-90 (2014). DOI: 10.1146/annurev-matsci070813-113447.

[Cah90] D. G. Cahill. "Thermal conductivity measurement from 30 to $750 \mathrm{~K}$ : the $3 \omega$ method“. In: Rev. Sci. Instrum. 61, 802-808 (1990). DOI: 10.1063/1. 1141498 .

[Car65] M. Cardona. „Optical Properties and Band Structure of $\mathrm{SrTiO}_{3}$ and Ba$\mathrm{TiO}_{3}$ “. In: Phys. Rev. 140, A651-A655 (1965). DOI: 10.1103/PhysRev. 140 A651. 
[Che+08] Y. Chen et al. „Polaron formation in the optimally doped ferromagnetic

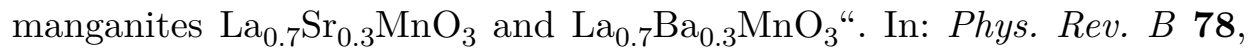
212301 (2008). DOI: 10.1103/PhysRevB.78.212301.

[Cho+06] T.-Y. Choi, D. Poulikakos, J. Tharian und U. Sennhauser. „Measurement of the Thermal Conductivity of Individual Carbon Nanotubes by the FourPoint Three-w Method“. In: Nano Lett. 6, 1589-1593 (2006). DoI: 10.1021/ n1060331v,

[CKA94] D. G. Cahill, M. Katiyar und J. R. Abelson. „Thermal conductivity of a-Si:H thin films". In: Phys. Rev. B 50, 6077-6081 (1994). DOI: 10.1103/ PhysRevB.50.6077.

[Dab+98] B. Dabrowski et al. „Synthesis and properties of the vacancy-free $\mathrm{La}_{1-x} \mathrm{Ba}_{x}$ $\mathrm{MnO}_{3}{ }^{“}$. In: Phys. Rev. B 58, 2716-2723 (1998). DOI: 10.1103/PhysRevB 58.2716 .

[Dag03] E. Dagotto. Nanoscale Phase Separation and Colossal Magnetoresistance: The Physics of Manganites and Related Compounds. Springer Series in Solid-State Sciences. Springer Berlin Heidelberg, 2003. DOI: 10.1007/9783-662-05244-0.

[Dag05] E. Dagotto. „Complexity in Strongly Correlated Electronic Systems“. In: Science 309, 257-262 (2005). DOI: 10.1126/science.1107559

$[$ Dao+02] A. Daoud-Aladine, J. Rodríguez-Carvajal, L. Pinsard-Gaudart, M. T. Fernández-Díaz und A. Revcolevschi. „Zener Polaron Ordering in Half-Doped Manganites“. In: Phys. Rev. Lett. 89, 097205 (2002). DOI: 10.1103/ PhysRevLett.89.097205

[Dem07] W. Demtröder. Laserspektroskopie 1: Grundlagen und Techniken. 5. Auflage. Springer Berlin Heidelberg, 2007. DOI: 10.1007/978-3-642-21306-9.

[Des+16] S. B. Desai et al. „MoS $\mathrm{MoS}_{2}$ transistors with 1-nanometer gate lengths“. In: Science 354, 99-102 (2016). DOI: 10.1126/science.aah4698.

[DHM01] E. Dagotto, T. Hotta und A. Moreo. „Colossal magnetoresistant materials: the key role of phase separation". In: Phys. Rep. 344, 1-153 (2001). DOI: 10.1016/S0370-1573(00)00121-6.

[Dor+05] V. Dorosinets, P. Richter, E. Mohler, H. G. Roskos und G. Jakob. „Picosecond energy relaxation in $\mathrm{La}_{0.67} \mathrm{Ca}_{0.33} \mathrm{MnO}_{3}$ ". In: Physica B 359-361, 1297-1299 (2005). DOI: 10.1016/j .physb.2005.01.362.

[Fie+00] M. Fiebig, K. Miyano, Y. Tomioka und Y. Tokura. „Sub-picosecond photoinduced melting of a charge-ordered state in a perovskite manganite". In: Appl. Phys. B 71, 211-215 (2000). DOI: 10.1007/s003400000338.

[Fie+98] M. Fiebig, K. Miyano, Y. Tomioka und Y. Tokura. „Visualization of the Local Insulator-Metal Transition in $\mathrm{Pr}_{0.7} \mathrm{Ca}_{0.3} \mathrm{MnO}_{3}{ }^{*}$. In: Science 280, 19251928 (1998). DOI: $10.1126 /$ science.280.5371.1925 
[Geh+10] K. Gehrke et al. „Interface controlled electronic variations in correlated heterostructures“. In: Phys. Rev. B 82, 113101 (2010). DOI: 10.1103/ PhysRevB.82.113101

[Gen60] P.-G. de Gennes. „Effects of Double Exchange in Magnetic Crystals“. In: Phys. Rev. 118, 141-154 (1960). DOI: 10.1103/PhysRev.118.141.

[GJJ76] L. A. Guildner, D. P. Johnson und F. E. Jones. „Vapor pressure of water at its triple point". In: J. Res. Natl. Bur. Stand. A 80, 505-521 (1976). DOI: 10.6028/jres.080A.054.

[Gol26] V. M. Goldschmidt. „Die Gesetze der Krystallochemie“. In: Naturwissenschaften 14, 477-485 (1926). DOI: 10.1007/BF01507527.

[Goo55] J. B. Goodenough. „Theory of the Role of Covalence in the Perovskite-Type Manganites [La,M(II)] $\mathrm{MnO}_{3}$ “. In: Phys. Rev. 100, 564-573 (1955). DOI: 10.1103/PhysRev.100.564

[Gro04] R. Gross. Spinelektronik. Vorlesungsskript. Walther-Meißner-Institut, Technische Universität München. 2004.

[GWT03] R. Y. Gu, Z. D. Wang und C. S. Ting. „Theory of electric-field-induced metal-insulator transition in doped manganites". In: Phys. Rev. B 67, 153101 (2003). DOI: 10.1103/PhysRevB.67.153101.

$[\mathrm{Hel}+93] \quad$ R. von Helmolt, J. Wecker, B. Holzapfel, L. Schultz und K. Samwer. „Giant negative magnetoresistance in perovskitelike $\mathrm{La}_{2 / 3} \mathrm{Ba}_{1 / 3} \mathrm{MnO}_{x}$ ferromagnetic films". In: Phys. Rev. Lett. 71, 2331-2333 (1993). Dor: 10.1103/ PhysRevLett.71.2331.

[Hüh10] S. Hühn. „Präparation und Eigenschaften von dünnen $\left(\mathrm{La}_{1-y} \mathrm{Pr}_{y}\right)_{0.67} \mathrm{Ca}_{0.33}$ $\mathrm{MnO}_{3}$ Schichten“. Diplomarbeit. Georg-August-Universität Göttingen, 2010.

[Hwa+95] H. Y. Hwang, S.-W. Cheong, P. G. Radaelli, M. Marezio und B. Batlogg. "Lattice Effects on the Magnetoresistance in Doped $\mathrm{LaMnO}_{3}$ “. In: Phys. Rev. Lett. 75, 914-917 (1995). DOI: 10.1103/PhysRevLett.75.914.

[Jia+08] W. Jiang, X. Zhou, G. Williams, Y. Mukovskii und K. Glazyrin. „Griffiths phase and critical behavior in single-crystal $\mathrm{La}_{0.7} \mathrm{Ba}_{0.3} \mathrm{MnO}_{3}$ : Phase diagram for $\mathrm{La}_{1-x} \mathrm{Ba}_{x} \mathrm{MnO}_{3}(x \leqslant 0.33) “$. In: Phys. Rev. B 77, 064424 (2008). DOI: 10.1103/PhysRevB.77.064424.

[Jin+94] S. Jin et al. „Thousandfold Change in Resistivity in Magnetoresistive LaCa-Mn-O Films". In: Science 264, 413-415 (1994). DOI: 10.1126/science 264.5157 .413 .

[JT37] H. A. Jahn und E. Teller. „Stability of polyatomic molecules in degenerate electronic states. I. Orbital degeneracy“. In: Proc. R. Soc. London, Ser. A. Bd. 161. 905. The Royal Society. 1937, S. 220-235. DOI: 10.1098/rspa 1937.0142 
$[\mathrm{Ju}+00] \quad$ H. L. Ju, Y. S. Nam, J. E. Lee und H. S. Shin. „Anomalous magnetic properties and magnetic phase diagram of $\mathrm{La}_{1-x} \mathrm{Ba}_{x} \mathrm{MnO}_{3}$ “. In: J. Magn. Magn. Mater. 219, 1-8 (2000). DOI: 10.1016/S0304-8853(00)00429-7.

[Jun+14] M. Jungbauer, S. Hühn, J.-O. Krisponeit und V. Moshnyaga. „Visible range colossal magnetorefractive effect in $\left(\mathrm{La}_{1-y} \mathrm{Pr}_{y}\right)_{2 / 3} \mathrm{Ca}_{1 / 3} \mathrm{MnO}_{3}$ films". In: New J. Phys. 16, 063034 (2014). DOI: 10.1088/1367-2630/16/6/063034.

[Jun+98] J. H. Jung, K. H. Kim, T. W. Noh, E. J. Choi und J. Yu. „Midgap states of $\mathrm{La}_{1-x} \mathrm{Ca}_{x} \mathrm{MnO}_{3}$ : Doping-dependent optical-conductivity studies“. In: Phys. Rev. B 57, R11043-R11046 (1998). DOI: 10.1103/PhysRevB.57.R11043.

[Jun15] M. Jungbauer. „Gestaltung der elektronischen Korrelationen in PerowskitHeterostrukturen auf atomarer Skala“. Dissertation. Georg-August-Universität Göttingen, 2015.

[JV50] G. Jonker und J. Van Santen. „Ferromagnetic compounds of manganese with perovskite structure“. In: Physica 16, 337-349 (1950). DOI: 10.1016/00318914(50)90033-4

[Kah76] D. Kahng. „A historical perspective on the development of MOS transistors and related devices". In: IEEE Trans. Electron Devices 23, 655-657 (1976). DOI: 10.1109/T-ED.1976.18468.

[Kal+11] C. Kalkert et al. „Resistive switching at manganite/manganite interfaces“. In: Appl. Phys. Lett. 99, 132512 (2011). DOI: 10.1063/1.3643425.

[Kal13] C. Kalkert. „Elektrischer Transport in Manganatschichten: Einfluss von elektrischen Feldern und Licht“. Dissertation. Georg-August-Universität Göttingen, 2013.

[Kan59] J. Kanamori. „Superexchange interaction and symmetry properties of electron orbitals". In: J. Phys. Chem. Solids 10, 87-98 (1959). DoI: 10.1016/ 0022-3697(59)90061-7.

[KFN99] J. H. Kim, A. Feldman und D. Novotny. „Application of the three omega thermal conductivity measurement method to a film on a substrate of finite thickness". In: J. Appl. Phys. 86, 3959-3963 (1999). DOI: 10.1063/1 371314 .

[Kin06] A. Kingon. „Perovskites: Is the ultimate memory in sight?“ In: Nat. Mater. 5, 251-252 (2006). DOI: 10.1038/nmat1623.

[Kir+03] V. Kiryukhin, T. Y. Koo, H. Ishibashi, J. P. Hill und S.-W. Cheong. „Average lattice symmetry and nanoscale structural correlations in magnetoresistive manganites". In: Phys. Rev. B 67, 064421 (2003). DOI: 10.1103/PhysRevB 67.064421 .

[KK82] K. I. Kugel' und D. I. Khomskiı̌. „The Jahn-Teller effect and magnetism: transition metal compounds". In: Sov. Phys. Usp. 25, 231 (1982). DOI: 10.1070/PU1982v025n04ABEH004537. 
$\left[\right.$ Kov+10] N. N. Kovaleva et al. „Low-energy Mott-Hubbard excitations in $\mathrm{LaMnO}_{3}$ probed by optical ellipsometry“. In: Phys. Rev. B 81, 235130 (2010). DOI: 10.1103/PhysRevB.81.235130

[KSB00] H. Kawaura, T. Sakamoto und T. Baba. „Observation of source-to-drain direct tunneling current in $8 \mathrm{~nm}$ gate electrically variable shallow junction metal-oxide-semiconductor field-effect transistors". In: Appl. Phys. Lett. 76, 3810-3812 (2000). DOI: 10.1063/1.126789.

[Kuz15] A. Kuzmenko. Guide to RefFIT: software to fit optical spectra. 2015. URL: http://optics.unige.ch/alexey/reffit.html

[LC97] S.-M. Lee und D. G. Cahill. „Heat transport in thin dielectric films“. In: $J$. Appl. Phys. 81, 2590-2595 (1997). DOI: 10.1063/1.363923

$[$ Leo+10] I. Leonov, D. Korotin, N. Binggeli, V. I. Anisimov und D. Vollhardt. „Computation of correlation-induced atomic displacements and structural transformations in paramagnetic $\mathrm{KCuF}_{3}$ and $\mathrm{LaMnO}_{3}$ ". In: Phys. Rev. B 81, 075109 (2010). DOI: 10.1103/PhysRevB.81.075109.

[Lou+97] D. Louca, T. Egami, E. L. Brosha, H. Röder und A. R. Bishop. „Local JahnTeller distortion in $\mathrm{La}_{1-x} \mathrm{Sr}_{x} \mathrm{MnO}_{3}$ observed by pulsed neutron diffraction". In: Phys. Rev. B 56, R8475-R8478 (1997). DOI: 10.1103/PhysRevB. 56 R8475.

[LTA03] A. N. Lavrov, I. Tsukada und Y. Ando. „Normal-state conductivity in underdoped $\mathrm{La}_{2-x} \mathrm{Sr}_{x} \mathrm{CuO}_{4}$ thin films: Search for nonlinear effects related to collective stripe motion". In: Phys. Rev. B 68, 094506 (2003). DOI: 10.1103/PhysRevB.68.094506.

[Lui+11] M. Luisier, M. Lundstrom, D. A. Antoniadis und J. Bokor. „Ultimate device scaling: Intrinsic performance comparisons of carbon-based, InGaAs, and Si field-effect transistors for $5 \mathrm{~nm}$ gate length“. In: Electron Devices Meeting (IEDM), 2011 IEEE International. 2011, S. 11.2.1-11.2.4. DOI: 10.1109/IEDM.2011.6131531

[Lun03] M. Lundstrom. „Moore's Law Forever?“ In: Science 299, 210-211 (2003). DOI: $10.1126 /$ science.1079567.

[May+01] M. Mayr et al. „Resistivity of Mixed-Phase Manganites“. In: Phys. Rev. Lett. 86, 135-138 (2001). DOI: 10.1103/PhysRevLett.86.135.

[MG03] P. Mandal und B. Ghosh. „Transport, magnetic, and structural properties of $\mathrm{La}_{1-x} M_{x} \mathrm{MnO}_{3}(M=\mathrm{Ba}, \mathrm{Sr}, \mathrm{Ca})$ for $0 \leqslant x \leqslant 0.20 “$. In: Phys. Rev. B 68, 014422 (2003). DOI: 10.1103/PhysRevB.68.014422.

[MI08] K. Momma und F. Izumi. „VESTA: a three-dimensional visualization system for electronic and structural analysis". In: J. Appl. Crystallogr. 41, 653-658 (2008). DOI: $10.1107 /$ S0021889808012016. 
[Mil+15] S. Mildner, J. Hoffmann, P. E. Blöchl, S. Techert und C. Jooss. „Temperatureand doping-dependent optical absorption in the small-polaron system $\operatorname{Pr}_{1-x}$ $\mathrm{Ca}_{x} \mathrm{MnO}_{3}{ }^{*} . \mathrm{In}$ : Phys. Rev. B 92, 035145 (2015). DOI: 10.1103/PhysRevB 92.035145 .

[Mil96] A. J. Millis. „Cooperative Jahn-Teller effect and electron-phonon coupling in $\mathrm{La}_{1-x} A_{x} \mathrm{MnO}_{3}$ “. In: Phys. Rev. B 53, 8434-8441 (1996). DOI: 10.1103/ PhysRevB.53.8434.

$[$ Miy +97$] \quad$ K. Miyano, T. Tanaka, Y. Tomioka und Y. Tokura. „Photoinduced Insulatorto-Metal Transition in a Perovskite Manganite“. In: Phys. Rev. Lett. 78, 4257-4260 (1997). DOI: 10.1103/PhysRevLett.78.4257.

[ML03] N. Mathur und P. Littlewood. „Mesoscopic texture in manganites“. In: Phys. Today 56, 25-30 (2003). DOI: 10.1063/1.1554133.

[MLS95] A. J. Millis, P. B. Littlewood und B. I. Shraiman. „Double Exchange Alone Does Not Explain the Resistivity of $\mathrm{La}_{1-x} \mathrm{Sr}_{x} \mathrm{MnO}_{3}$ ". In: Phys. Rev. Lett. 74, 5144-5147 (1995). DOI: 10.1103/PhysRevLett.74.5144.

[Mos+00] V. Moshnyaga et al. „Intrinsic and extrinsic pressure effects in $\mathrm{La}_{0.7} \mathrm{Ca}_{0.3}$ $\mathrm{MnO}_{3}$ thin films“. In: J. Appl. Phys. 88, 5305-5310 (2000). DOI: 10.1063/ 1.1313778 .

[Mos+03] V. Moshnyaga, B. Damaschke, R. Tidecks und K. Samwer. „Low-field transverse magnetovoltage in manganite films". In: J. Appl. Phys. 93, 63546360 (2003). DOI: $10.1063 / 1.1567036$.

[Mos+04] V. Moshnyaga et al. „Giant negative photoconductivity in $\mathrm{La}_{0.7} \mathrm{Ca}_{0.3} \mathrm{MnO}_{3}$ thin films". In: J. Appl. Phys. 95, 7360-7362 (2004). DOI: 10.1063/1 1687555 .

[Mos+09] V. Moshnyaga et al. „Electrical nonlinearity in colossal magnetoresistance manganite films: Relevance of correlated polarons". In: Phys. Rev. B 79, 134413 (2009). DOI: 10.1103/PhysRevB.79.134413.

[Mos+10] V. Moshnyaga et al. „Memristor Behaviour in Nano-Sized Vertical Lsmo/Lsmo Tunnel Junctions“. In: ArXiv e-prints (2010).

[Mos+14] V. Moshnyaga et al. „Intrinsic antiferromagnetic coupling underlies colossal magnetoresistance effect: Role of correlated polarons". In: Phys. Rev. B 89, 024420 (2014). DOI: 10.1103/PhysRevB.89.024420.

[Mos+99] V. Moshnyaga et al. „Preparation of rare-earth manganite-oxide thin films by metalorganic aerosol deposition technique“. In: Appl. Phys. Lett. 74, 2842-2844 (1999). DOI: 10.1063/1.124032.

[Mou+99] N. Moutis, I. Panagiotopoulos, M. Pissas und D. Niarchos. „Structural and magnetic properties of $\mathrm{La}_{0.67}\left(\mathrm{Ba}_{x} \mathrm{Ca}_{1-x}\right)_{0.33} \mathrm{MnO}_{3}$ perovskites $(0 \leqslant x \leqslant 1)^{\text {“. }}$. In: Phys. Rev. B 59, 1129-1133 (1999). DOI: 10.1103/PhysRevB.59.1129. 
[MS07] V. Moshnyaga und K. Samwer. „Ferromagnetic Manganite Films“. In: Handbook of Magnetism and Advanced Magnetic Materials. Hrsg. von H. Kronmüller und S. Parkin. John Wiley \& Sons, Ltd, 2007. DOI: 10.1002/ 9780470022184.hmm504.

[MS11] V. Moshnyaga und K. Samwer. „Electron-lattice correlations and phase transitions in CMR manganites“. In: Ann. Phys. 523, 652-663 (2011). DOI: 10.1002/andp.201100040

[Mül+09] G. M. Müller et al. „Spin polarization in half-metals probed by femtosecond spin excitation". In: Nat. Mater. 8, 56-61 (2009). DOI: 10.1038/nmat2341

[Mül88] E. Müller-Hartmann. „Überlegungen zur Theorie der Hochtemperatursupraleiter". In: 19. IFF-Ferienkurs: Supraleitung und verwandte Quantenphänomene. Kernforschungsanlage Jülich GmbH, 1988, S. 28.1-28.20.

[Mur+98] Y. Murakami et al. „Resonant X-Ray Scattering from Orbital Ordering in $\mathrm{LaMnO}_{3}$ “. In: Phys. Rev. Lett. 81, 582-585 (1998). DoI: 10.1103/ PhysRevLett.81.582

[Nel+01] C. S. Nelson et al. „Correlated polarons in dissimilar perovskite manganites“. In: Phys. Rev. B 64, 174405 (2001). DOI: 10.1103/PhysRevB.64.174405.

[Oga+03] T. Ogasawara et al. „Photoinduced spin dynamics in $\mathrm{La}_{0.6} \mathrm{Sr}_{0.4} \mathrm{MnO}_{3}$ observed by time-resolved magneto-optical Kerr spectroscopy". In: Phys. Rev. B 68, 180407 (2003). DOI: 10.1103/PhysRevB.68.180407.

$[\mathrm{Oh}+11] \quad$ D.-W. Oh et al. „Thermal conductivity as a metric for the crystalline quality of SrTiO3 epitaxial layers". In: Appl. Phys. Lett. 98, 221904 (2011). DOI: $10.1063 / 1.3579993$

[Oki+95] Y. Okimoto et al. „Anomalous Variation of Optical Spectra with Spin Polarization in Double-Exchange Ferromagnet: $\mathrm{La}_{1-x} \mathrm{Sr}_{x} \mathrm{MnO}_{3}$ “. In: Phys. Rev. Lett. 75, 109-112 (1995). DOI: 10.1103/PhysRevLett.75.109

[Oki+99] Y. Okimoto, Y. Tomioka, Y. Onose, Y. Otsuka und Y. Tokura. „Optical study of $\operatorname{Pr}_{1-x} \mathrm{Ca}_{x} \mathrm{MnO}_{3}(x=0.4)$ in a magnetic field: Variation of electronic structure with charge ordering and disordering phase transitions". In: Phys. Rev. B 59, 7401-7408 (1999). DOI: 10.1103/PhysRevB.59.7401.

$[\mathrm{Pad}+04]$ P. Padhan, W. Prellier, C. Simon und R. C. Budhani. „Current-induced metallic behavior in $\operatorname{Pr}_{0.5} \mathrm{Ca}_{0.5} \mathrm{MnO}_{3}$ thin films: Competition between Joule heating and nonlinear conduction mechanisms". In: Phys. Rev. B 70, 134403 (2004). DOI: 10.1103/PhysRevB.70.134403.

$[\mathrm{Pfa}+17] \quad \mathrm{V}$. Pfahl et al. „Conduction electrons as dissipation channel in friction experiments at the metal-metal transition of LSMO measured by contactresonance atomic force microscopy". In: Appl. Phys. Lett. 110, 053102 (2017). DOI: $10.1063 / 1.4975072$. 
[Qua07] Quantum Design. Physical Property Measurement System, Hardware Manual. 5th edition. 2007.

[Qui+98] M. Quijada et al. „Optical conductivity of manganites: Crossover from JahnTeller small polaron to coherent transport in the ferromagnetic state". In: Phys. Rev. B 58, 16093-16102 (1998). DOI: 10.1103/PhysRevB.58.16093.

[RA96] L. M. Rodriguez-Martinez und J. P. Attfield. „Cation disorder and size effects in magnetoresistive manganese oxide perovskites". In: Phys. Rev. B 54, R15622-R15625 (1996). DOI: 10.1103/PhysRevB.54.R15622.

[Rad +97$]$ P. G. Radaelli, D. E. Cox, M. Marezio und S.-W. Cheong. „Charge, orbital, and magnetic ordering in $\mathrm{La}_{0.5} \mathrm{Ca}_{0.5} \mathrm{MnO}_{3}{ }^{*}$. In: Phys. Rev. B 55, 3015-3023 (1997). DOI: 10.1103/PhysRevB.55.3015.

[Rai+17] D. Raiser et al. „Evolution of Hot Polaron States with a Nanosecond Lifetime in a Manganite Perovskite“. In: Adv. Energy Mater. 1602174 (2017). DOI: 10.1002/aenm. 201602174

[Ram97] A. P. Ramirez. „Colossal magnetoresistance“. In: J. Phys.: Condens. Matter 9, 8171 (1997). DOI: 10.1088/0953-8984/9/39/005.

[Ren+04] Y. H. Ren et al. „Ultrafast photoinduced reflectivity transients in doped manganite". In: J. Chem. Phys. 121, 436-440 (2004). DOI: 10.1063/1 1755661 .

[Ren+08] Y. H. Ren et al. „Time-resolved optical studies of spin and quasiparticle dynamics in colossal magnetoresistance materials: $\mathrm{La}_{0.67} \mathrm{Ca}_{0.33} \mathrm{MnO}_{3}$, $\mathrm{La}_{0.67} \mathrm{Sr}_{0.33} \mathrm{MnO}_{3}$, and $\mathrm{Sr}_{2} \mathrm{FeMoO}_{6}$ ". In: Phys. Rev. B 78, 014408 (2008). DOI: $10.1103 /$ PhysRevB.78.014408.

[Rey+07] N. Reyren et al. „Superconducting Interfaces Between Insulating Oxides“. In: Science 317, 1196-1199 (2007). DOI: 10.1126/science.1146006.

[Rin+07] M. Rini et al. „Control of the electronic phase of a manganite by modeselective vibrational excitation“. In: Nature 449, 72-74 (2007). DOI: 10 1038/nature06119.

[Ros97] I. M. Ross. "The foundation of the silicon age“. In: Bell Labs Tech. J. 2, 3-14 (1997). DOI: 10.1002/bltj. 2080.

[RV11] P. Ramachandran und G. Varoquaux. „Mayavi: 3D Visualization of Scientific Data“. In: Comput. Sci. Eng. 13, 40-51 (2011). DOI: 10.1109/MCSE. 2011 35.

[SBH09] G. Singh-Bhalla, A. Biswas und A. F. Hebard. „Tunneling magnetoresistance in phase-separated manganite nanobridges". In: Phys. Rev. B 80, 144410 (2009). DOI: 10.1103/PhysRevB.80.144410.

[Sch15] M. Scherff. „Elektrischer Transport und remanentes Widerstandsschalten in $\mathrm{Pt}-\mathrm{Pr}_{0.7} \mathrm{Ca}_{0.3} \mathrm{MnO}_{3}-\mathrm{Pt}$ Sandwichstrukturen“. Dissertation. GeorgAugust-Universität Göttingen, 2015. 
[Sch16] K. Schwab. The Fourth Industrial Revolution. World Economic Forum, 2016.

[Sha76] R. D. Shannon. „Revised effective ionic radii and systematic studies of interatomic distances in halides and chalcogenides". In: Acta Crystallogr., Sect. A 32, 751-767 (1976). DOI: 10.1107/S0567739476001551.

$[$ Tak+00] K. Takenaka, Y. Sawaki, R. Shiozaki und S. Sugai. „Electronic structure of the double-exchange ferromagnet $\mathrm{La}_{0.825} \mathrm{Sr}_{0.175} \mathrm{MnO}_{3}$ studied by optical reflectivity“. In: Phys. Rev. B 62, 13864-13867 (2000). DOI: $10.1103 /$ PhysRevB.62.13864

[TT99] Y. Tokura und Y. Tomioka. „Colossal magnetoresistive manganites“. In: J. Magn. Magn. Mater. 200, 1-23 (1999). DOI: 10.1016/S0304-8853(99) 00352-2.

[Ueh+99] M. Uehara, S. Mori, C. H. Chen und S.-W. Cheong. „Percolative phase separation underlies colossal magnetoresistance in mixed-valent manganites". In: Nature 399, 560-563 (1999). DOI: 10.1038/21142.

[Ven+98] T. Venkatesan, M. Rajeswari, Z. i-Wen Dong, S. B. Ogale und R. Ramesh. „Manganite-based devices: opportunities, bottlenecks and challenges". In: Philos. Trans. R. Soc. London, Ser. A 356, 1661-1680 (1998). DOI: 10 1098/rsta.1998.0240.

[WA59] A. Wold und R. J. Arnott. „Preparation and crystallographic properties of the systems $\mathrm{LaMn}_{1-x} \mathrm{Mn}_{x} \mathrm{O}_{3+\lambda}$ and $\mathrm{LaMn}_{1-x} \mathrm{Ni}_{x} \mathrm{O}_{3+\lambda}$ ". In: J. Phys. Chem. Solids 9, 176-180 (1959). DOI: 10.1016/0022-3697(59)90207-0

$[$ Wan +08$]$ Z. Wang et al. „Spin dynamics triggered by subterahertz magnetic field pulses“. In: J. Appl. Phys. 103, 123905 (2008). DOI: 10.1063/1.2940734.

[WB08] S. G. Warren und R. E. Brandt. „Optical constants of ice from the ultraviolet to the microwave: A revised compilation“. In: J. Geophys. Res. Atmos. 113, D14220 (2008). DOI: 10.1029/2007JD009744.

[Wes+06] W. Westhäuser, S. Schramm, J. Hoffmann und C. Jooss. „Comparative study of magnetic and electric field induced insulator-metal-transitions in $\mathrm{Pr}_{1-x} \mathrm{Ca}_{x} \mathrm{MnO}_{3}$ films". In: Eur. Phys. J. B 53, 323-331 (2006). DOI: 10.1140/epjb/e2006-00387-2.

$[\mathrm{Wu}+09] \quad \mathrm{K} . \mathrm{H}$. Wu et al. „Ultrafast optical probes of polaron dynamics in $\mathrm{La}_{0.7} \mathrm{Ca}_{0.3}$ $\mathrm{MnO}_{3}$ thin films". In: J. Appl. Phys. 105, 043901 (2009). DOI: 10.1063/1. 3078082 .

[Zen51] C. Zener. „Interaction between the $d$-Shells in the Transition Metals. II. Ferromagnetic Compounds of Manganese with Perovskite Structure". In: Phys. Rev. 82, 403-405 (1951). DOI: 10.1103/PhysRev.82.403.

[Zha+06] H.-Y. Zhai et al. „Giant Discrete Steps in Metal-Insulator Transition in Perovskite Manganite Wires". In: Phys. Rev. Lett. 97, 167201 (2006). DOI: 10.1103/PhysRevLett.97.167201. 
[Zha+98] Y. G. Zhao et al. „Ultrafast Laser Induced Conductive and Resistive Transients in $\mathrm{La}_{0.7} \mathrm{Ca}_{0.3} \mathrm{MnO}_{3}$ : Charge Transfer and Relaxation Dynamics“. In: Phys. Rev. Lett. 81, 1310-1313 (1998). DOI: 10.1103/PhysRevLett. 81 1310 .

[ZSA85] J. Zaanen, G. A. Sawatzky und J. W. Allen. „Band gaps and electronic structure of transition-metal compounds". In: Phys. Rev. Lett. 55, 418-421 (1985). DOI: 10.1103/PhysRevLett.55.418. 


\section{Publikationen}

\section{Artikel in wissenschaftlichen Fachzeitschriften}

- Electron spin resonance of the $\mathrm{Yb} 4 f$-moment in $\mathrm{Yb}\left(\mathrm{Rh}_{1-x} \mathrm{Fe}_{x}\right)_{2} \mathrm{Si}_{2}$ J. Sichelschmidt, H. S. Jeevan, M. Mchalwat, P. Gegenwart Physica Status Solidi B, 250: 495-497 (2013)

- Multiple Metamagnetic Quantum Criticality in $\mathrm{Sr}_{3} \mathrm{Ru}_{2} \mathrm{O}_{7}$ Y. Tokiwa, M. Mchalwat, R. S. Perry, P. Gegenwart

Physical Review Letters 116, 226402 (2016)

- Laser-induced changes of non-linear electric transport properties in $\mathrm{La}_{0.75} \mathrm{Ba}_{0.25}$ $\mathrm{MnO}_{3}$ and $\left(\mathrm{La}_{0.6} \mathrm{Pr}_{0.4}\right)_{0.67} \mathrm{Ca}_{0.33} \mathrm{MnO}_{3}$ thin films

M. Mchalwat, H. Ulrichs, J. Walowski, C. Ballani, O. Shapoval, V. Moshnyaga, M. Müzenberg, K. Samwer (eingereicht)

\section{Beiträge auf Konferenzen}

- Frühjahrstagung der Deutschen Physikalischen Gesellschaft, Dresden, 2011

Poster: Quantum criticality in $\mathrm{Sr}_{3} \mathrm{Ru}_{2} \mathrm{O}_{7}$ studied by measurements of the magnetocaloric effect

- Frühjahrstagung der Deutschen Physikalischen Gesellschaft, Berlin, 2012

Poster: Effect of hole doping and chemical pressure in $\mathrm{Yb}\left(\mathrm{Rh}_{1-x} \mathrm{Fe}_{x}\right)_{2} \mathrm{Si}_{2}$

- Frühjahrstagung der Deutschen Physikalischen Gesellschaft, Regensburg, 2013

Poster: Autocorrelation measurement with femtosecond laser pulses of correlated polaron dynamics in manganites

Vortrag: Photoinduced changes of the first and third harmonic voltage at the metal insulator transition

- Frühjahrstagung der Deutschen Physikalischen Gesellschaft, Dresden, 2014

Poster: Time evolution of laser-induced changes in electric transport of manganites

- Frühjahrstagung der Deutschen Physikalischen Gesellschaft, Berlin, 2015

Poster: Laser-induced changes of electronic transport properties in manganites 



\section{Danksagung}

Abschließend möchte ich vielen Menschen, die mich während meiner Doktorarbeit unterstützt und begleitet haben, dafür Danke sagen, zunächst Prof. Dr. KonrAD Samwer und Prof. Dr. Markus Münzenberg für das Ermöglichen dieser Arbeit, die fachlichen Diskussionen, Ideen und Anmerkungen. Weiter geht ein besonders großer Dank an Prof. Dr. Vasily Moshnyaga, der mir in unzähligen Gesprächen und Diskussion die Manganatphysik sehr weit veranschaulicht hat und mit seinen Ideen und Anregungen die Entwicklung zur Erklärung der beobachteten Phänomene maßgeblich vorangetrieben hat.

Für die fachliche Unterstützung im Laboralltag und die intensiven und äußerst tiefgehenden Diskussionen über Hintergründe und Ursachen der Messergebnisse danke ich besonders Dr. Henning UlRichs und ebenso Dr. JAKOB WALOWSKi für seine Hilfe bei den Messungen in Greifswald.

Meinen Kollegen und Freunden Markus Jungbauer, Victor Pfahl und CamilLO BALlANi sage ich vielmals Dank für viele fachliche, klärende Diskussionen, fürs Korrekturlesen, aber auch für intensive Gespräche über fachfremde Dinge und spaßige Freizeitaktivitäten wie z. B. Kneipentouren, Mountainbiketouren und Umzugstouren. $:-\mathrm{D}$

Weiterhin sage ich der Manganatgruppe Danke für das gemeinsame Arbeiten, Diskutieren und Ergründen an dieser Materialklasse.

Ich danke Carsten Mahn und Uta Filippich und dem technischen Personal für vielerlei Hilfe und Ratschläge, sowohl technische als auch persönliche; PROF. DR. KLAUS Winzer für die aufschlussreichen Gespräche über die Feinheiten elektronischer Messtechnik und der Feinmechanikwerkstatt des I. Physikalischen Instituts und der Zentralwerkstatt der Fakultät für Physik für die tatkräftige Unterstützung beim Bau des Messeinsatzes; ebenso Dr. Bernd Damaschke für das Korrekturlesen der Arbeit.

Zusammenfassend möchte ich dem gesamten I. Physikalischen Institut mit seinen gegenwärtigen und ehemaligen Mitgliedern innig danken. Über die kumulierte Zeit der Bachelor-, Master- und Doktorarbeit ist das Institut für mich wie eine zweite Familie und ein zweites Zuhause geworden. Tatsächlich kann Letzteres sogar wörtlich verstanden werden, denn die meiste Zeit vom Tag habe ich fast immer, auch öfter an Wochenenden, im Institut verbracht. Der dortige enge Zusammenhalt, der freundliche Umgang, die Zusammenarbeit, die Diskussionskultur und die Hilfsbereitschaft und die gemeinsamen Unternehmungen, sei es auf Tagungen, Ausflügen, der Weihnachtsfeier oder beim gemütlichen Grillen im Sommer, sind wahrhaft herausragend und ich kann mir keine angenehmere Arbeits- und Wissenschaftsumgebung vorstellen. Diese Gemeinschaft werde ich - und das kann ich jetzt schon mit Sicherheit sagen - sehr vermissen. 
Mein größter Dank allerdings, was eigentlich nicht in Worte zu fassen ist, gilt meiner Familie. Ohne ihren Rückhalt, ohne die Rückzugsmöglichkeiten, ohne die vielen Familienfeiern und sonntäglichen Kaffeetafeln in großer Runde mit unzähligen Diskussionen über wichtige oder eher unwichtige Dinge ;-) wäre es mir nicht möglich gewesen, durchzuhalten. Nochmals unendlichen Dank dafür.

Zum Schluss liegt mir noch am Herzen, diese Arbeit meinen vier Großeltern Oma Dörchen, Opa Fritz, Oma Nado und Opa Mounir zu widmen. Meine Oma und mein Opa mütterlicherseits haben mich seit meiner jüngsten Kindheit geprägt und begleitet und ihre Fürsorge und Nähe war und ist ein Geschenk, welches unbezahlbar ist. Von meinen anderen Großeltern durfte ich meine Oma, obwohl sie immer weit weg war, noch kennenlernen, was leider bei meinem Opa nicht mehr möglich war. Aber ich trage seinen Namen und hoffe, dass er ein wenig stolz auf das Geleistete sein kann. 